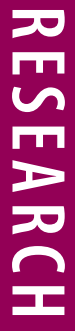

Marko Kovic

Agenda-Setting

zwischen Parlament

und Medien

Normative Herleitung

und empirische Untersuchung

am Beispiel der Schweiz 
Agenda-Setting zwischen Parlament und Medien 
Marko Kovic

\section{Agenda-Setting \\ zwischen Parlament \\ und Medien}

Normative Herleitung

und empirische Untersuchung

am Beispiel der Schweiz

Mit einem Geleitwort von Prof. Dr. Heinz Bonfadelli 
Marko Kovic

Zürich, Schweiz

Die vorliegende Arbeit wurde von der Philosophischen Fakultät der Universität Zürich im Herbstsemester 2015 auf Antrag der Promotionskommission, bestehend aus Prof. Dr. Heinz Bonfadelli (hauptverantwortliche Betreuungsperson) und Prof. Dr. Werner A. Meier, als Dissertation angenommen.

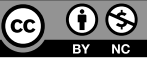

ISBN 978-3-658-15897-2

ISBN 978-3-658-15898-9 (eBook)

DOI 10.1007/978-3-658-15898-9

Die Deutsche Nationalbibliothek verzeichnet diese Publikation in der Deutschen Nationalbibliografie; detaillierte bibliografische Daten sind im Internet über http://dnb.d-nb.de abrufbar.

Springer VS

(C) Der/die Herausgeber bzw. der/die Autor(en) 2017. Dieses Buch ist eine Open-Access-Publikation. Open Access Dieses Buch wird unter der Creative Commons Namensnennung - Nicht kommerziell 4.0 International Lizenz (http://creativecommons.org/licenses/by-nc/4.0/deed. de) veröffentlicht, welche für nicht kommerzielle Zwecke die Nutzung, Vervielfältigung, Bearbeitung, Verbreitung und Wiedergabe in jeglichem Medium und Format erlaubt, sofern Sie den/die ursprünglichen Autor(en) und die Quelle ordnungsgemäß nennen, einen Link zur Creative Commons Lizenz beifügen und angeben, ob Änderungen vorgenommen wurden.

Etwaige Abbildungen oder sonstiges Drittmaterial unterliegen ebenfalls der genannten Creative Commons Lizenz, sofern sich aus der Abbildungslegende oder der Quellreferenz nichts anderes ergibt. Sofern solches Drittmaterial nicht unter der genannten Creative Commons Lizenz steht, ist eine Vervielfältigung, Bearbeitung oder öffentliche Wiedergabe nur mit vorheriger Zustimmung des betreffenden Rechteinhabers oder auf der Grundlage einschlägiger gesetzlicher Erlaubnisvorschriften zulässig.

Das Werk einschließlich aller seiner Teile ist urheberrechtlich geschützt. Jede kommerzielle Verwertung, die nicht ausdrücklich vom Urheberrechtsgesetz zugelassen ist, bedarf der vorherigen Zustimmung des Verlags. Das gilt insbesondere für Vervielfältigungen, Bearbeitungen, Übersetzungen, Mikroverfilmungen und die Einspeicherung und Verarbeitung in elektronischen Systemen.

Die Wiedergabe von Gebrauchsnamen, Handelsnamen, Warenbezeichnungen usw. in diesem Werk berechtigt auch ohne besondere Kennzeichnung nicht zu der Annahme, dass solche Namen im Sinne der Warenzeichen- und Markenschutz-Gesetzgebung als frei zu betrachten wären und daher von jedermann benutzt werden dürften.

Der Verlag, die Autoren und die Herausgeber gehen davon aus, dass die Angaben und Informationen in diesem Werk zum Zeitpunkt der Veröffentlichung vollständig und korrekt sind. Weder der Verlag, noch die Autoren oder die Herausgeber übernehmen, ausdrücklich oder implizit, Gewähr für den Inhalt des Werkes, etwaige Fehler oder Äußerungen.

Gedruckt auf säurefreiem und chlorfrei gebleichtem Papier

Springer VS ist Teil von Springer Nature

Die eingetragene Gesellschaft ist Springer Fachmedien Wiesbaden GmbH

Die Anschrift der Gesellschaft ist: Abraham-Lincoln-Str. 46, 65189 Wiesbaden, Germany 
Meinen Eltern 


\section{Geleitwort}

Die vorliegende Dissertation von Marko Kovic entstand im Rahmen des Sinergia-Projekts «Medienkrise und Medienwandel» des Schweizerischen Nationalfonds zur Förderung der wissenschaftlichen Forschung SNF sowie des integrierten Doktorandenprogramms, geleitet von Prof. Dr. Heinz Bonfadelli und Prof. Dr. Werner A. Meier vom IPMZ - Institut für Publizistikwissenschaft und Medienforschung der Universität Zürich. Vor dem Hintergrund der schon länger andauernden Finanzierungskrise vor allem der Zeitungen im Gefolge der Verbreitung des Internets, sahen sich die Verlage nicht nur in der Schweiz gezwungen, sich zum einen redaktionell stärker im Internet zu engagieren, und zum anderen zwecks Einsparung von Kosten im journalistischen Bereich abzubauen, etwa durch die Einführung von sog. integrierten Newsrooms. In diesem Kontext stellen sich verschiedenste Fragen, nicht zuletzt nach den Konsequenzen für die journalistischen Leistungen der Medien.

Die Dissertation von Marko Kovic befasst sich mit der für die Demokratie speziell wichtigen Frage der Parlamentsberichterstattung. Im Zentrum der Arbeit stehen die beiden Fragen: 1) Über welche Themen bzw. Geschäfte des Schweizerischen Parlaments berichten die Medien und in welchem Ausmass? 2) Darüber hinaus wurde untersucht, inwiefern die Medienberichterstattung selbst wiederum das Handeln der Parlamentarier beeinflusst. Diese Fragen sind insofern gesellschaftlich und demokratietheoretisch relevant, als politisch informierte Bürger die Voraussetzung für das Funktionieren der Demokratie sind.

Empirisch umgesetzt wurden die beiden forschungsleitenden Fragestellungen mittels einer standardisierten Inhaltsanalyse sowohl der Vorgänge im Parlament als auch der darauf bezogenen Medienberichterstattung. Die Auswertung der inhaltsanalytischen Daten wurden im Sinne einer Triangulation ergänzt durch achtzehn Leitfadengespräche mit Parlamentariern und zusätzlichen acht Interviews mit Medienschaffenden. Dabei stellt sich nicht zuletzt die Frage, wie die sog. Medien-Logik die Parlamentsberichterstattung beeinflusst, aber auch, ob Parlamentarier selber ihr Handeln verstärkt sich an der Medien-Logik und weniger an der Politik-Logik orientieren.

Die vielfältigen und interessanten Befunde weisen auf komplexe wechselseitige Zusammenhänge hin, wobei nicht zuletzt der jeweilige thematische Kontext bzw. der konkrete politische Sachverhalt entscheidend sowohl für die Berichterstattung der Medien als auch beispielsweise für die Vorstösse der Parlamentarier und ihr Verhalten in den Fragestunden sein dürften.

Prof. Dr. Heinz Bonfadelli 


\section{Danksagung}

Ganz herzlich danke ich meinen beiden Betreuern, Prof. Dr. Heinz Bonfadelli und Prof. Dr. Werner A. Meier. Sie haben den bemerkenswerten Spagat gemeistert, mich in turbulenteren, wenn nicht gar krisenartigen, Arbeitsphasen zu unterstützen, mir gleichzeitig aber auch grossen kreativen Forschungsfreiraum zu ermöglichen. Ein solches Vertrauen ist nicht selbstverständlich.

Zu grossem Dank bin ich auch Dr. Martina Leonarz verpflichtet; unter anderem, aber nicht nur, weil sie mir als operative Leiterin des Sinergia-Doktoratsprogrammes aus manch einer selbstverschuldeten organisatorischen Bredouille geholfen hat. Auch meinen studentischen Mitarbeitenden Sereina Caduff, Mirjam Hausherr, Aleksandra Hiltmann, Nils Weber und Clemens Schroeder gebührt grosser Dank. Ohne ihre intensive Mithilfe bei der Datenerhebung und -aufbereitung wäre die vorliegende Arbeit nicht möglich gewesen. 


\section{Inhaltsverzeichnis}

$\begin{array}{ll}\text { Geleitwort } & 7\end{array}$

$\begin{array}{ll}\text { Danksagung } & 9\end{array}$

$\begin{array}{ll}\text { Tabellenverzeichnis } & 15\end{array}$

$\begin{array}{ll}\text { Abbildungsverzeichnis } & 17\end{array}$

1 Einleitung $\quad 19$

1.1 Aufbau der Arbeit . . . . . . . . . . . . . . . . . . . . 22

2 Entdeckungszusammenhang $\quad 25$

2.1 Medien, Öffentlichkeit und das Parlament . . . . . . . . . . . . . . . . . 25

2.1.1 Zur Notwendigkeit des Entdeckungszusammenhanges . . . . . . . . 25

2.1.2 Der Sinn massenmedialer Öffentlichkeit . . . . . . . . . . . . . . . 26

2.1.3 Zweckrationales Handeln und Präferenzen . . . . . . . . . . . . . . 32

2.1.4 Die kommunikative Legitimität des Parlamentes . . . . . . . . . . . . . 35

2.2 Problemaufriss und Forschungsfragen . . . . . . . . . . . . . . . . . . 41

2.2.1 Medienwandel, Medienkrise und das Parlament . . . . . . . . . . . . 41

2.2.2 Die Schnittmengen der Politiklogiken und der Medienlogiken . . . . . 44

2.2 .3 Forschungsfragen . . . . . . . . . . . . . . . . 51

2.3 Forschungsstand . . . . . . . . . . . . . . . . . 53

2.3.1 Präferenzen der Parlamentarierinnen und Parlamentarier . . . . . . . . 55

2.3.2 Präferenzen der Medien . . . . . . . . . . . . . . . . 61

2.3.3 Wandel der Präferenzen von Parlament und Medien . . . . . . . . . . . 63

2.3.4 Fazit und Beitrag der vorliegenden Arbeit . . . . . . . . . . . . . . . 66

3 Theoretische Einbettung $\quad 69$

3.1 Agenda Setting . . . . . . . . . . . . . . . . . . . . . 69

3.1.1 Agenda Setting und die Politikagenda . . . . . . . . . . . . . . . 71

3.1.2 Theorie oder proto-theoretisches Framework? . . . . . . . . . . . . 72

3.2 Medialisierung . . . . . . . . . . . . . . . . . 74

3.2.1 Medialisierung oder Medialisiertheit? . . . . . . . . . . . . . . 78

3.2.2 Medialisierung und parlamentarisches Handeln . . . . . . . . . . . . 81

3.3 Rational Choice-Institutionalismus . . . . . . . . . . . . . . . . . . . . . . . 83 
3.3.1 Die Theorie nutzenmaxierender Akteure: Rational Choice . . . . . . . 83

3.3.2 «Institutions matter»: Rational Choice-Institutionalismus . . . . . . . 86

3.3.3 Rational Choice-Institutionalismus und parlamentarisches Handeln . . 87

3.4 Theoriesynthese und Hypothesen . . . . . . . . . . . . . . . . . . . . . . . 89

4 Forschungsdesign und Methoden $\quad 95$

4.1 Forschungsdesign . . . . . . . . . . . . . . . . . . . . . . . 95

4.2 Datenerhebung. . . . . . . . . . . . . . . . . . 97

4.2 .1 Inhaltsanalyse . . . . . . . . . . . . . . . . . . 97

4.2 .2 Leitfadeninterviews . . . . . . . . . . . . . . . . . . . . . . . 106

4.3 Datenauswertung . . . . . . . . . . . . . . . . . . . . 111

4.3.1 Auswertung der Inhaltsanalyse . . . . . . . . . . . . . . . . . . . 111

4.3.2 Auswertung der Interviews . . . . . . . . . . . . . . . . . . . . . . . 113

4.3.3 Zusammenführen der Ergebnisse . . . . . . . . . . . . . . . . . . . 113

4.4 Schwächen des Designs und der Methoden . . . . . . . . . . . . . . . . 115

5 Ergebnisse und Diskussion $\quad 119$

5.1 Ergebnisse . . . . . . . . . . . . . . . . . . . . . . . . 119

5.1.1 H1 und H2: Medieneinfluss auf Abstimmungsverhalten . . . . . . . . 120

5.1.2 H3, H4 und H5: Medieneinfluss auf Vorstösse und Fragestunden . . . . 128

5.1.3 H6: Politiklogiken und Medienlogiken im Parlament . . . . . . . . . . 137

5.1.4 H7: Ziele medialisierten Handelns im Parlament . . . . . . . . . . . . . 139

5.1.5 H8 und H9: Politiklogiken und Medienlogiken in den Medien . . . . . 149

5.1.6 Zusammenführung der Ergebnisse . . . . . . . . . . . . . . . . . . . 160

5.2 Diskussion . . . . . . . . . . . . . . . . . . . . . . . . . 162

5.2.1 Forschungsfrage 1: Präferenzen im Parlament . . . . . . . . . . . . . 162

5.2.2 Forschungsfrage 2: Präferenzen in den Medien . . . . . . . . . . . . . 163

5.2 .3 Forschungsfrage 3: Wandel . . . . . . . . . . . . . . . . . . . . 164

6 Schlussbetrachtungen $\quad 171$

6.1 Zusammenfassung der Ergebnisse . . . . . . . . . . . . . . . . . . 171

6.2 Normative Einschätzung der Ergebnisse . . . . . . . . . . . . . . . . . . . . 175

6.2.1 Die praktische Relevanz des Normativen . . . . . . . . . . . . . . . 177

6.3 Weiterführende Forschung . . . . . . . . . . . . . . . . . . . . . . 182

6.3.1 Wessen Agent? Präferenzen und Lobbying . . . . . . . . . . . . . . . 184 
6.4 Praxisrelevanz der Ergebnisse . . . . . . . . . . . . . . . . . . . 188

6.4.1 Analyseschritt 1: Identifikation der betroffenen Stakeholder . . . . . . 191

6.4.2 Analyseschritt 2: Erkenntnisse für die betroffenen Stakeholder . . . . . 192

6.4.3 Analyseschritt 3: Ausformulieren der Handlungsempfehlungen . . . . 194

6.4.4 Umsetzungsschritt: Implementierung, Monitoring und Evaluation . . . 198

$\begin{array}{llr}7 & \text { Literatur } & 201\end{array}$

$\begin{array}{lr}\text { A Anhang } & 229\end{array}$ 


\section{Tabellenverzeichnis}

Tabelle 1 Forschung zu Medienkrise / Medienwandel und Parlament . . . . . . . . . 64

Tabelle 2 Erhebungs- und Untersuchungseinheiten für Thema und Politikfeld . . . . 98

Tabelle 3 Liste der verwendeten Politikfelder . . . . . . . . . . . . . . . . . 101

Tabelle 4 Sessionen für die Inhaltsanalyse . . . . . . . . . . . . . . . . . . 103

Tabelle 5 Zeiträume für die Medienstichproben . . . . . . . . . . . . . . . 104

Tabelle 6 Medienstichproben . . . . . . . . . . . . . . . . . 104

Tabelle 7 Anzahl Untersuchungseinheiten in der Inhaltsanalyse . . . . . . . . . . 105

Tabelle 8 Anzahl und Zugehörigkeiten Interviewpartner . . . . . . . . . . . . . . 108

Tabelle 9 Parteizugehörigkeiten Interviewpartner National- und Ständerat . . . . . 108

Tabelle 10 Geschlechterverteilung Interviewpartner . . . . . . . . . . . . . . . . 108

Tabelle 11 Methoden zur Prüfung der Hypothesen . . . . . . . . . . . . . . . . . . 114

Tabelle 12 Anzahl der untersuchten Abstimmungen . . . . . . . . . . . . . . . . . . 120

Tabelle 13 Abstimmungsverhalten und Berichterstattungsumfang . . . . . . . . . . . 122

Tabelle 14 Vorstösse und Berichterstattung 1999 . . . . . . . . . . . . . . . . . . . . 129

Tabelle 15 Vorstösse und Berichterstattung $2005 \ldots \ldots \ldots$. . . . . . . . . . 130

Tabelle 16 Vorstösse und Berichterstattung $2011 \ldots \ldots$. . . . . . . . . . . 130

Tabelle 17 Einfluss Medien auf Fragestunden $1999 \ldots \ldots$. . . . . . . . . . . . . . 131

Tabelle 18 Einfluss Medien auf Fragestunden 2005 . . . . . . . . . . . . . . . . . . . 132

Tabelle 19 Einfluss Medien auf Fragestunden $2011 \ldots$. . . . . . . . . . . . . . . . . 133

Tabelle 20 Zusammenfassung der Hypothesen H1 bis H5 . . . . . . . . . . . . . . . 137

Tabelle 21 Berichterstattung zu NR, SR, Vorstössen 1999 . . . . . . . . . . . . . . . 150

Tabelle 22 Berichterstattung zu NR, SR, Vorstössen 2005 . . . . . . . . . . . . . . . 151

Tabelle 23 Berichterstattung zu NR, SR, Vorstössen 2011 . . . . . . . . . . . . . 152

Tabelle 24 Berichterstattung zu Fragestunden . . . . . . . . . . . . . . . . . . 152

Tabelle 25 Parlamentsberichterstattung $1999 \ldots \ldots$. . . . . . . . . . . . . . . 154

Tabelle 26 Parlamentsberichterstattung $2005 \ldots \ldots \ldots$. . . . . . . . . . 155

Tabelle 27 Parlamentsberichterstattung $2011 \ldots \ldots$. . . . . . . . . . . . 156

Tabelle 28 Zusammenfassung der Hypothesen H1 bis H9 . . . . . . . . . . . . . . 161

Tabelle 29 Variablen für die Inhaltsanalyse der Zeitungen . . . . . . . . . . . . . . . 233

Tabelle 30 Ausprägungen und Codes der Variable Autorschaft. . . . . . . . . . . . . . 236

Tabelle 31 Ausprägungen der Variable Artikeltyp . . . . . . . . . . . . . . . . . 236

Tabelle 32 Ausprägungen der Variable Artikelgrösse . . . . . . . . . . . . . . . 237 
Tabelle 33 Ausprägungen der Variable Bildgrösse . . . . . . . . . . . . . . . . . 238

Tabelle 34 Ausprägungen der Variable Politikfeld . . . . . . . . . . . . . . . . . . . 241

Tabelle 35 Ausprägungen der Variable Partei . . . . . . . . . . . . . . . . . . . . . 247

Tabelle 36 Ausprägungen der Variable Rolle . . . . . . . . . . . . . . . . . . . . . . 247

Tabelle 37 Ausprägungen der Variable Geschlecht . . . . . . . . . . . . . . . . . . . 248

Tabelle 38 Variablen für das Codieren der Tagesschau . . . . . . . . . . . . . . . 250

Tabelle 39 Ausprägungen der Variable Beitragstypen . . . . . . . . . . . . . . . 250

Tabelle 40 Variablen im amtlichen Bulletin . . . . . . . . . . . . . . . . . . . 255

Tabelle 41 Ausprägungen für die Variable Form . . . . . . . . . . . . . . . . . . 256

Tabelle 42 Ausprägungen für die Variable Sprache . . . . . . . . . . . . . . . . . . 256

Tabelle 43 Ausprägungen für die Variable Abstimmungsart . . . . . . . . . . . . . . 258

Tabelle 44 Variablen für die eingereichten Vorstösse . . . . . . . . . . . . . . . . . . . 261

Tabelle 45 Ausprägungen der Variable Vorstossart . . . . . . . . . . . . . . . . . . . 261

Tabelle 46 Ausprägungen der Variable Kammer . . . . . . . . . . . . . . . . . . . 262

Tabelle 47 Variablen für die Fragestunden . . . . . . . . . . . . . . . . . . . 263

Tabelle 48 Ausprägungen der Variable Rolle . . . . . . . . . . . . . . . . . . . . . 264 


\section{Abbildungsverzeichnis}

Abbildung $1 \quad$ Werbeeinnahmen Print, Rundfunk, Online 1982 bis 2013 . . . . . . . 42

Abbildung $2 \quad$ Auflagezahlen Zeitungen 2003 bis $2014 \ldots \ldots$. . . . . . . . . . . 43

Abbildung 3 Präferenzen von Wählerschaft, Parlament, Medien . . . . . . . . . . 46

Abbildung $4 \quad$ Politiklogiken und Medienlogiken . . . . . . . . . . . . . . 48

Abbildung 5 Abstimmungen und Berichterstattung 1999 . . . . . . . . . . . 123

Abbildung 6 Abstimmungen und Berichterstattung 2005 . . . . . . . . . . . . . 124

Abbildung $7 \quad$ Abstimmungen und Berichterstattung 2011 . . . . . . . . . . . . . 125

Abbildung 8 Präferenzen Wählerschaft, Parlament, Medien, Lobbygruppen .... 187 


\section{Einleitung}

Jede moderne staatliche Ordnung, für welche der Anspruch erhoben wird, es handle sich um eine demokratische Ordnung, muss dem Prinzip der Volkssouveränität in genügendem Masse Folge leisten. Moderne Demokratien sehen sich entsprechend mit der Herausforderung konfrontiert, die theoretisch-normative Rolle der Volkssouveränität als Ausdruck des vernunftgeleiteten kollektiven Willens individueller Bürgerinnen und Bürger empirisch umzusetzen. Als mögliche Ausprägungen von Volkssouveränität werden bisweilen denn auch unterschiedliche institutionelle Arrangements subsumiert, im Schweizer Kontext etwa Momente direktdemokratischer Entscheidungsfindung über Initiativen und Referenden, in einem allgemeineren Kontext die Möglichkeit, zu wählen und gewählt zu werden. Wenn auch Volkssouveränität grundsätzlich in Form unterschiedlicher Institutionen und über diese Institutionen stattfindende Verfahren umgesetzt werden kann, gibt es eine Kerninstitution, welche die notwendige Bedingung für Volkssouveränität und damit für Demokratie darstellt: das Parlament. Die Institution des Parlamentes ist nämlich der einzige Ort, an welchem Volkssouveränität nicht nur punktuelle, sondern stetige empirische Umsetzung findet. Parlamentarierinnen und Parlamentarier werden über Wahlen eingesetzt, um im Auftrag der Bürgerinnen und Bürger, durch welche sie gewählt wurden, die Aufgabe der Gesetzgebung wahrzunehmen. Somit wird Volkssouveränität über die Institution des Parlamentes nicht bloss über die direkten, aber nur periodisch stattfindenden Entscheidungsmomente der Parlamentswahlen wahrgenommen, sondern beständig indirekt durch das Vorhandensein des Parlamentes an und für sich - dadurch, dass es die Institution des Parlamentes gibt, legiferiert das souveräne Volk immerwährend indirekt über die Repräsentantinnen und Repräsentanten im Parlament.

Ein Parlament ist allerdings nur dann demokratisch legitim, wenn bestimmte Bedingungen erfüllt sind. $\mathrm{Zu}$ diesen Bedingungen gehören etwa freie und faire Wahlen, oder auch die verfassungsrechtliche Unabhängigkeit des Parlamentes von der Regierung. Die Legitimität des Parlamentes ergibt sich aber nicht nur aus solchen formaljuristischen Kriterien, denn auch ein latentes Kriterium, die öffentliche Beobachtbarkeit des Parlamentes, ist eine Notwendigkeit für dessen demokratische Legitimität. Das Parlament ist nicht nur dadurch demokratisch legitim, dass Bürgerinnen und Bürger die Volkssouveränität periodisch über Wahlen wahrnehmen, sondern zusätzlich dadurch, dass das Parlament für die Bürgerinnen und Bürger in den Phasen zwischen den Wahlen öffentlich sichtbar ist. Bürgerinnen und Bürger müssen also prinzipiell die Möglichkeit haben, die Arbeit des Parlamentes beobachten zu können. Erst dann nämlich, wenn die Arbeit des Parlamentes öffentlich beobachtbar ist, macht die Annahme, dass die 
Wahlentscheidungen der Bürgerinnen und Bürgern reflektierte, sinngeleitete Handlungen sind und die Wahlen somit tatsächlich den Willen des Volkes darstellen, Sinn. Wenn wir in einem fiktiven Beispiel davon ausgehen, dass das maximal mögliche Mass, in welchem Bürgerinnen und Bürger die Arbeit des Parlamentes beobachten können, bei Null liegt, dass das Parlament also als politischer Arkanraum operiert, dann ist es den Bürgerinnen und Bürgern auch nicht möglich, einzuschätzen, ob und in welchem Masse jene Arbeit, welche die von ihnen gewählten Personen und Parteien im Parlament leisten, mit der Arbeit, welche die Bürgerinnen und Bürger geleistet sehen wollen, übereinstimmt. Öffentliche Kommunikation rund um das Parlament bildet somit die Brücke zwischen dem Parlament und den Bürgerinnen und Bürgern, und durch diese kommunikative Brücke ensteht das demokratisch unabdingbare Rechenschaftsverhältnis zwischen dem Parlament und den Bürgerinnen und Bürgern.

Die Frage nach der öffentlichen Sichtbarkeit des Parlamentes erschöpft sich nicht in dem dichotomen Kriterium der prinzipiellen Sichtbarkeit oder Unsichtbarkeit. Wenn das Parlament nämlich grundsätzlich öffentlich sichtbar ist, stellt sich die Anschlussfrage, in welchem Masse und in welcher Güte diese Sichtbarkeit gegeben ist. Hier spielen eine Reihe von Dimensionen eine Rolle, beispielsweise eine logistische Dimension (wie viele individuelle Bürgerinnen und Bürger können effektiv die Sessionen im Bundeshaus in Echtzeit mitverfolgen), eine personelle Dimension (wie viele Ressourcen sind vorhanden, um die Arbeit des Parlamentes während Sessionen in Parlamentsprotokollen zu dokumentieren), und so fort. In der vorliegenden Arbeit fokussiere ich auf die Dimension der massenmedialen öffentlichen Kommunikation, also auf jene Kommunikation rund um das Parlament, welche durch professionelle Journalistinnen und Journalisten bei Zeitungen, Fernsehen und Radio hergestellt wird. Massenmediale Kommunikation über das Parlament erachte ich als die wichtigste Dimension der Öffentlichkeit des Parlamentes, weil eine wirklich gesellschaftsweite, also den ganzen politischen Geltungsraum abdeckende Beobachtbarkeit des Parlamentes erst durch Massenmedien ermöglicht wird. In dieser Arbeit untersuche ich die massenmediale Kommunikation rund um das Parlament in der Schweiz. Konkret widme ich mich der Frage, wie sich die Bundesversammlung und Medien während einer Session gegenseitig beeinflussen. Das bedeutet, dass ich einerseits untersuche, über welche Inhalte des Parlamentes - die Geschäfte des National- und des Ständerates, die eingereichten Vorstösse sowie die Fragen in den Fragestunden - Medien in welchem Masse berichten. Andererseits untersuche ich auch, ob und in welchem Umfang bestimmte Aspekte des Parlamentes - das Abstimmungsverhalten im National- und im Ständerat, die eingereichten Vorstösse sowie die Fragen in den Fragestunden - durch die Inhalte der Medien beeinflusst werden. Diese Wechselwirkungen analysiere ich aus dem Blickwinkel des Agenda Setting. 
Um diese Wechselwirkungen nicht nur deskriptiv abzubilden, sondern darüber hinaus auch handlungstheoretisch zu verstehen, konzipiere ich sie als Handlungen individueller Akteure im Parlament (Parlamentarierinnen und Parlamentarier) sowie als Handlungen individueller Akteure in den Medien (Journalistinnen und Journalisten) im Lichte der Theorien von Medialisierung und Rational Choice-Institutionalismus. Die Akteure im Parlament und in den Medien verfolgen unterschiedliche Ziele und handeln auf eine solche Art, dass sie auf das Erreichen dieser Ziele hinarbeiten. Mit der Untersuchung der Wechselwirkungen zwischen Parlament und Medien will ich verstehen, inwiefern die inhaltlichen Wechselwirkungen zwischen Parlament und Medien das Ergebnis dieses Hinarbeitens auf bestimmte Ziele seitens der involvierten Akteure sind. Genauer geht es mir darum, auf der Grundlage des theoretischen Verständnisses zielorientiert handelnder Akteure zu verstehen, worin genau diese Ziele der involvierten Akteure bestehen. Diese Ziele - im Laufe der Arbeit verwende ich auch den Begriff der Präferenzen um auszudrücken, dass die Akteure bestimmte Ziele eher erreichen möchten als andere - können, erstens, ganz konkrete spezifische Ausprägungen haben. Beispielsweise kann eine Parlamentarierin ein aktuelles Thema aus den Medien aufgreifen und einen Vorstoss dazu einreichen, weil sie der aufrichtigen Überzeugung ist, dass das Thema wichtig ist. Handkehrum kann eine Journalistin über einen beliebigen eingereichten Vorstoss berichten, weil sie der aufrichtigen Überzeugung ist, dass der Vorstoss politisch wichtig ist. Die spezifischen individuellen Ziele der Akteure im Parlament und in den Medien im Kontext der massenmedialen Kommunikation über das Parlament können aber, zweitens, auch auf einer verallgemeinerten Ebene beschrieben werden, nämlich anhand des Kriteriums, ob die spezifischen Ziele eher den generellen Zielen der Politik (Politiklogiken) oder eher den generellen Zielen der Medien (Medienlogiken) entsprechen. Mit der vorliegenden Arbeit möchte ich in diesem Sinne erklären, in welchem Masse das Handeln der Parlamentarierinnen und Parlamentarier sowie der Journalistinnen und Journalisten den zwei Familien von Zielen der Politiklogiken und der Medienlogiken entspricht.

Die Wechselwirkungen zwischen Parlament und Medien während der Session erachte ich nicht als universale Konstante, also als System, welches unabhängig von Zeit und gesellschaftlichem Kontext nach den stets gleichen Regeln abläuft. Im Gegenteil: Ganz explizit untersuche ich nicht bloss, nach welchen Zielen die Akteure im Parlament und in den Medien handeln, sondern darüber hinaus auch, ob sich ihre Ziele und damit ihr Handeln im Zuge der Medienkrise verändert. Seit ca. Anfang der 2000er Jahre finden sich Medien mit der Tatsache konfrontiert, dass der Zugang zu und die Nutzung des Internets stetig zunehmen und, dass mit der Nutzung des Internets eine starke Veränderung der Mediennutzung einhergeht. Klassische Trägerme- 
dien journalistischer Inhalte (Print, Fernsehen und Radio) werden weniger rezipiert, doch Medienhäuser finden keine nachhaltigen Wege, um ihre Online-Inhalte in denselbem Umfang zu monetarisieren wie die über die klassischen Trägermedien verbreiteten Inhalte. Dies führt zu einem Einbruch der Werbeeinnahmen vor allem bei Printmedien. Die durch Digitalisierung, Wandel des Nutzungsverhaltens, Wandel der Geschäftsmodelle und nicht zuletzt Wandel der Werbefinanzierung bedingte Medienkrise hat weitreichende Folgen auf der Makroebene (Medienkonzentration), der Mesoebene (Veränderung redaktioneller Strukturen und Demografien) sowie der Mikroebene (Veränderungen des journalistischen Outputs). Für die vorliegende Arbeit ist es darum von Interesse, ob und wie sich das Handeln von Parlamentarierinnen und Parlamentariern sowie von Journalistinnen und Journalisten im Kontext der massenmedialen Kommunikation über das Parlament im Zuge der Medienkrise verändert.

Um diesen Fragestellungen empirisch nachzugehen, wende ich das ForschungsdesignParadigma der Triangulation an: Mit unterschiedlichen Methoden der Datenerhebung und Datenauswertung analysiere ich unterschiedliche Untersuchungseinheiten, um damit die Fragestellungen aus unterschiedlichen Perspektiven auszuleuchten. Konkret setze ich die Idee der Triangulation mit zwei separaten Teilanalysen um. Zum einen untersuche ich inhaltsanalytisch, welche Themen in welchem Umfang Gegenstand welcher parlamentarischen Teilarenen sind, sowie, welche Themen in welchem Umfang Gegenstand der politischen Berichterstattung einer Medienstichprobe sind. Die Medienstichprobe besteht aus einer Fernsehsendung, einer Radiosendung sowie aus acht Tages- und Sonntagszeitungen. Zum anderen untersuche ich mittels teilstrukturierter Interviews mit Parlamentarierinnen und Parlamentariern sowie mit Journalistinnen und Journalisten, wie die betroffenen Akteure selber die Wechselwirkungen wahrnehmen und, mit was für Zielen sie ihr jeweiliges Handeln im Kontext dieser Wechselwirkungen verbinden.

\subsection{Aufbau der Arbeit}

Die Arbeit ist in fünf Teile gegliedert. In Abschnitt 2 erkläre ich den Entdeckungszusammenhang der Arbeit. Zunächst begründe ich in Abschnitt 2.1, warum und wie das Verhältnis vom Parlament im Allgmeinen, der Schweizer Bundesversammlung im Besonderen zu Medien bzw. zu massenmedial hergestellter Öffentlichkeit relevant ist. Anschliessend konkretisiere ich diese allgemeine Relevanz in Abschnitt 2.2 zu einer spezifischeren Problemstellung und formuliere zu diesem Zweck die Forschungsfragen, welche ich mit dieser Arbeit beantworten möchte. Den gegenwärtigen Forschungsstand und den Beitrag der vorliegenden Arbeit angesichts dieses Forschungsstandes bespreche ich in Abschnitt 2.3. 
In Abschnitt 3 beschreibe ich genauer, aus welchen theoretischen Perspektiven ich die in Abschnitt 2 formulierten Forschungsfragen zu beantworten gedenke. Dazu bespreche ich zunächst die theoretischen Ansätze des Agenda Setting in Unterabschnitt 3.1, der Medialisierung in Unterabschnitt 3.2 sowie des Rational Choice-Insitutionalismus in Abschnitt 3.3, um in Unterabschnitt $3.4 \mathrm{zu}$ begründen, wie ich diese drei theoretischen Perspektiven miteinander verbinde und aus ihnen Hypothesen ableite.

Abschnitt 4 ist dem Forschungsdesign und den verwendeten Methoden gewidmet. In Unterabschnitt 4.1 beschreibe ich zunächst den Designansatz der Triangulation. In Unterabschnitt 4.2 beschreibe ich die unterschiedlichen methodischen Herangehensweisen für die Erhebung der Daten, in Unterabschnitt 4.3 für deren Auswertung. Zuletzt reflektiere ich in Unterabschnitt 4.4 die Grenzen und Probleme des Designs und der Methoden.

Die empirischen Ergebnisse stelle ich Abschnitt 5 vor. Zunächst präsentiere ich in Unterabschnitt 5.1 die Ergebnisse für die einzelnen Hypothesen, um sie anschliessend in Unterabschnitt 5.2 für die Klärung der Forschungsfragen zusammenzuführen.

Im abschliessenden Abschnitt 6 fasse ich zunächst die Ergebnisse der Arbeit in Unterabschnitt 6.1 zusammen. Anschliessend evaluiere ich in Unterabschnitt 6.2 die Ergebnisse aus normativer Sicht. In Unterunterabschnitt 6.2.1 gehe ich der Frage nach, inwiefern die normative Perspektive dieser Arbeit mit den normativen Einschätzungen der betroffenen Akteure selber, also der Parlamentarierinnen und Parlamentarier sowie der Journalistinnen und Journalisten, übereinstimmt. Abschliessend bespreche ich in Unterabschnitt 6.3, wie weiterführende Forschung zu dem Themenbereich dieser Arbeit in praktischer und in theoretischer Hinsicht aussehen kann und soll, sowie in Unterabschnitt 6.4, wie es um die Praxisrelevanz der Ergebnisse steht.

Open Access Dieses Buch wird unter der Creative Commons Namensnennung - Nicht kommerziell 4.0 International Lizenz (http://creativecommons.org/licenses/by-nc/4.0/deed. de) veröffentlicht, welche für nicht kommerzielle Zwecke die Nutzung, Vervielfältigung, Bearbeitung, Verbreitung und Wiedergabe in jeglichem Medium und Format erlaubt, sofern Sie den/die ursprünglichen Autor(en) und die Quelle ordnungsgemäß nennen, einen Link zur Creative Commons Lizenz beifügen und angeben, ob Änderungen vorgenommen wurden.

Etwaige Abbildungen oder sonstiges Drittmaterial unterliegen ebenfalls der genannten Creative Commons Lizenz, sofern sich aus der Abbildungslegende oder der Quellreferenz nichts anderes ergibt. Sofern solches Drittmaterial nicht unter der genannten Creative Commons Lizenz steht, ist eine Vervielfältigung, Bearbeitung oder öffentliche Wiedergabe nur mit vorheriger Zustimmung des betreffenden Rechteinhabers oder auf der Grundlage einschlägiger gesetzlicher Erlaubnisvorschriften zulässig.

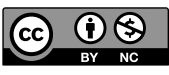




\section{Entdeckungszusammenhang}

\subsection{Medien, Öffentlichkeit und das Parlament}

\subsubsection{Zur Notwendigkeit des Entdeckungszusammenhanges}

[D]on't ask a barber if you need a haircut - and don't ask an academic if what he does is relevant. (Taleb 2010, S. 183)

In der vorliegenden Arbeit untersuche ich die Wechselwirkungen zwischen Medien und Parlament in der Schweiz. Es ist wenig überraschend, dass ich diesem Thema ein grosses Mass an Bedeutung zuschreibe - dass das Thema erforschenswert ist, ist für mich subjektiverweise geradezu selbstverständlich. Nicht selbstverständlich ist, dass meine subjektive Bedeutungsallokation deckungsgleich mit einer objektiven, oder zumindest intersubjektiv nachvollziehbaren, Einschätzung der Bedeutung der in dieser Arbeit untersuchten Thematik ist. Darum soll in den folgenden Abschnitten der Versuch unternommen werden, jene Überlegungen nachzuzeichnen, auf denen das in dieser Arbeit dokumentierte Forschungsvorhaben gründet. Das bedeutet, dass der Entdeckungszusammenhang (Howard 2006; Reichenbach 1938) zu beschreiben ist. Der Entdeckungszusammenhang ist in doppelter Hinsicht eine Besprechung wert. Zum einen wird damit, wie bereits argumentiert, eine Begründung geliefert, warum das, was in der vorliegenden Arbeit untersucht wird, untersuchenswert ist. Zum anderen ist aus einer wissenschaftstheoretischen Perspektive der Entdeckungszusammenhang als Begründung für Forschungsvorhaben bei sozialwissenschaftlicher Forschung, anders als bei naturwissenschaftlicher Forschung, notwendig. Wenn auch der Vorgang des Erkenntnisgewinns in den Sozial- und Naturwissenschaften empirisch gesehen nicht so unterschiedlich ist, wie bisweilen vielleicht angenommen wird (Cole 1983), unterscheiden sich die erkenntnistheoretischen Prämissen von Sozial- und Naturwissenschaft. Naturwissenschaft beobachtet Ursachen und Wirkungen, während Sozialwissenschaft eher mit den Konzepten von Motiven und des sinngeleiteten Handelns ${ }^{1}$ operiert (Winch 1990, S. 45-51). Sozialwissenschaftliche Forschung bedeutet letztlich also Sinnverstehen (Habermas 1982, S. 194-284). Zudem hat Sozialwissenschaft als «weiche Wissenschaft» anders als Naturwissenschaft nicht den Anspruch, Ursachen und Wirkungen verhältnismässig einfacher Systeme deterministisch und prognostisch zu analysieren, sondern, verhältnismässig komplexe und kontingente Systeme probabilistisch beschreibbar zu machen (Pigliucci 2010, S. 6-23). Das bedeutet, dass sozialwissenschaftliche Forschung nicht eo ipso

1 Von «sinngeleitetem Handeln» zu sprechen, ist ein Stück weit tautologisch, denn Handeln grenzt sich von blossem Verhalten gerade dadurch ab, dass es sinngeleitet ist (M. Weber 2006, S. 12). 
plausibel ist, sondern einer expliziten Begründung bedarf: Sozialwissenschaftliche Forschung ist ein Stück weit immer explorative Forschung, denn sozialwissenschaftliche Fragestellungen sind nicht direkt kausal-deterministisch aus dem bestehenden Wissenskorpus ableitbar, weil sie selber das Ergebnis einer sinnverstehenden Komplexitätsreduktion der sozialen Realität sind.

\subsubsection{Der Sinn massenmedialer Öffentlichkeit}

Die Überschrift dieses Unterabschnittes verrät, oder impliziert im Mindesten, vier Dinge. Erstens, dass ich davon ausgehe, dass es so etwas wie «Öffentlichkeit» gibt. Zweitens, dass ich Öffentlichkeit vor allem als durch Massenmedien hergestellte ${ }^{2}$ Öffentlichkeit verstehe. Drittens, dass massenmedial hergestellte Öffentlichkeit einen noch näher zu definierenden «Sinn» hat. Und viertens, dass ich nicht der erste Forscher bin, der diese drei Position vertritt; die Überschrift ist eine Anspielung auf Weßler, Peters und Habermas (2007).

Öffentlichkeit ist ein Konzept, das auf einer intuitiven Ebene eingängig ist. Dass es so etwas wie Öffentlichkeit gibt, ist für uns fast selbstverständlich; nicht zuletzt, weil wir fast selbstverständlich davon ausgehen, dass es so etwas wie Privatheit gibt - wenn etwas privat sein kann, muss im Gegenzug auch etwas öffentlich sein können. Diese Unterscheidung zwischen privat und öffentlich hat eine lange, auf die griechische Antike zurückgehende Tradition (Roy 1999; Weintraub 1997), und diese Tradition hat u.a. das westliche, demokratische Rechtsverständnis bedeutend geprägt (Horwitz 1982). Die Unterscheidung nach privat und öffentlich ist also de facto eine konstitutive Eigenschaft zeitgenössischer Gesellschaften. Diese Unterscheidung, welche die Funktion einer ordnenden gesellschaftlichen Heuristik ausübt, ist aber noch keine Definition. Es handelt sich dabei nämlich lediglich um eine ex negativo-Annäherung an Öffentlichkeit - die Öffentlichkeit ist jener Teil der Gesellschaft, welcher nicht unter das Private fällt - , aber nicht um eine Definition im eigentlichen Sinne. Alleine mit der Unterscheidung nach privat und öffentlich ist eine klare Negativdefinition von öffentlich nämlich noch nicht gegeben, da mit dieser Heuristik keine fixen, definierbaren Grenzen gezogen werden; die Grenzen zwischen privat und öffentlich sind Gegenstand gesellschaftlicher Aushandlungsprozesse (Solove 2002; Thompson 2011). Versuche, die Grenzen zwischen privat und öffentlich doch fix zu ziehen, können gar darin münden, dass Missstände innerhalb des Privaten wie auch innerhalb des Öffentlichen perpetuiert und legitimiert werden durch den Verweis, das jeweilige Problem sei «Privatsache» (Gavison 1992). Ein Öffentlichkeitsverständnis anhand der Heuristik privat vs. öffentlich ist also problembehaftet, da definitorisch ungenügend; Öffentlichkeit kann

«Herstellen» ist hier nicht als wertender, sondern als deskriptiver Begriff verwendet. 
mit einer blossen ex negativo-Annäherung nicht präzise genug gefasst werden. Aus diesem Grund ist eine eigenständige positive Definition von Öffentlichkeit nötig, welche jenseits der Heuristik privat vs. öffentlich operiert. Das Problem hierbei: Eine einheitliche und allgemein anerkannte positive Definition von Öffentlichkeit existiert nicht.

Das Konzept der Öffentlichkeit beschreibt kein empirisch manifestes Phänomen. Öffentlichkeit ist nicht ein konkreter Gegenstand, sonder eher eine Art «Phantom» (Lippmann 1927, S. 77), und dieses Phantom als die Konsequenz gesamtgesellschaftlicher Handlungen ist unorganisiert und formlos (Dewey 1954, S. 67). Damit ist das, was wir als Öffentlichkeit verstehen, fundamental flüchtig, also nicht strukturell verfestigt. Es ist zwar möglich, Öffentlichkeit in einem recht wörtlichen Sinn als physischen, strukturell zumindest im Prinzip klar messbaren Ort beschreibbar zu machen, etwa, wenn das Konzept der sogenannten Versammlungsöffentlichkeit (Gerhards 1998; Gerhards und Neidhardt 1990) in einem eher wortwörtlichen Sinn bemüht wird. Letztlich aber entsteht Öffentlichkeit aber nicht dadurch, dass bestimmte physische Kanäle gegeben sind (etwa ein öffentlicher Platz bei der Versammlungsöffentlichkeit). Öffentlichkeit besteht nicht aus den physischen Kanälen an und für sich, sondern Öffentlichkeit ist das, was über die wie auch immer gearteten physischen Kanäle vermittelt wird. Und das, was vermittelt wird, ist Kommunikation.

Eine viel zitierte Definition des flüchtigen Phantoms Öffentlichkeit als öffentlicher Kommunikation, welche auch die Grundlage des Öffentlichkeitsverständnisses dieser Arbeit darstellt, liefert Habermas (1992, S. 436):

Die Öffentlichkeit lässt sich am ehesten als Netzwerk für die Kommunikation von Inhalten und Stellungnahmen, also von Meinungen beschreiben; dabei werden die Kommunikationsflüsse so gefiltert und synthetisiert, dass sie sich zu themenspezifisch gebündelten öffentlichen Meinungen verdichten.

Diese Definition beinhaltet zwei wesentliche Aspekte, welche ich auch als Grundlage für das in dieser Arbeit verwendete Konzept von Öffentlichkeit verwende. Erstens ist Öffentlichkeit ein Netzwerk von Kommunikation. Das bedeutet, dass öffentliche Kommunikation eine solche Kommunikation ist, welche zumindest prinzipiell den Anspruch erhebt, alle Teilnehmerinnen und Teilnehmern der öffentlichen Kommunikation zu adressieren. Das wiederum bedeutet, dass öffentliche Kommunikation Kommunikation ist, welche an die Öffentlichkeit gerichtet ist. Das bedeutet weiter, dass öffentliche Kommunikation fundamental performativen Charakter hat: Dadurch, dass Kommunikation die Intention hat, die Öffentlichkeit zu adressieren, wird das, was kommuniziert wird, zu Öffentlichkeit. Dieser eigentümliche performative Charakter 
von Kommunikation ist denn auch das, was Kommunikation Handlungscharakter verleiht: Sprechen $^{3}$ transzendiert die blosse Lautäusserung und wird zu einer Sprechhandlung, oder einem Sprechakt (Habermas 1981, S. 44-61; Searle 1970, S. 22-53; Austin 1975, S. 1-11; Searle 2006; Habermas 1976), dadurch, dass wir gegenüber der Öffentlichkeit den Anspruch erheben, dass das, was wir sagen, Gültigkeit hat ${ }^{4}$.

Der zweite wichtige Aspekt der Definition von Öffentlichkeit ist die Idee der «gebündelten» Kommunikation. Wenn auch Kommunikationsbündel nur eine Metapher sind, ist es eine nützliche Metapher, die an die Idee öffentlicher Kommunikation als Netzwerk anknüpft. Öffentliche Kommunikation als performative Handlung entsteht dadurch, dass wir unsere Kommunikation and den «fiktiven» Adressaten Öffentlichkeit richten. Nun bedeutet Öffentlichkeit in einem zweiten Schritt, dass wir uns nicht nur generell an die Öffentlichkeit richten, sondern, dass darüber hinaus die Themen, mit denen wir mir uns an die Öffentlichkeit richten, (zumindest ein Stück weit) auch Themen sind, mit denen sich andere Diskursteilnehmende ebenfalls an die Öffentlichkeit richten. In dieser Lesart bedeuten «Kommunikationsbündel», dass wir über bestimmte Themen nicht nur öffentlich sprechen, sondern, dass wir über bestimmte Themen miteinander öffentlich sprechen.

Öffentlichkeit bedeutet also an die Öffentlichkeit gerichtete Kommunikation, in welcher Themen nicht nur atomistisch mitgeteilt, sondern auch sozial diskutiert werden. Oben ist erwähnt, dass solche öffentliche Kommunikation über unterschiedliche Kanäle vermittelt werden kann. Diese unterschiedlichen Kanäle unterscheiden sich nach einem zentralen Kriterium: Der Rolle der Örtlichkeit (oder, anders ausgedrückt: der Geografie). Bestimmte Öffentlichkeitsformen, etwa die oben erwähnte Versammlungsöffentlichkeit, sind abhänagig von geografischen Gegebenheiten. Das bedeutet ganz banal, dass es Einschränkungen gibt, um an solchen Formen öffentlicher Kommunikation teilzunehmen (es ist nötig, zu einer bestimmten Zeit an einem bestimmten Ort zu sein). Andere Formen von Öffentlichkeit heben die Limitation des Örtlichen auf, indem die Kommunikation auf eine solche Art vermittelt wird, dass Ortsgebundenheit kein Kriterium mehr ist. Die wichtigste Form dieser ortsungebundenen öffentlichen Kommunikation findet über Massenmedien statt (Saxer 2012, S. 569). Massenmedien als Organisationen (auf der Mesoebene) und als Journalistinnen und Journalisten (auf der Mikroebene) widmen sich exklusiv dem öffentlichen Kommunizieren im Sinne der Selektion, Interpretation und Darstellung

$3 \quad$ Bildlich als pars pro toto für den Vorgang des Kommunizierens allgemein verstanden.

4 Im engeren Sinn kann Sprechen Handeln bedeuten, indem das, was ausgedrückt wird, dadurch, dass es ausgedrückt wird, sozial wahr wird - das sind, im engeren Sinn, performative Sprechakte. In einem breiteren Sinn kann Sprechen Handeln bedeuten, indem dadurch, dass etwas gesagt wird, der Anspruch erhoben wird, dass die Bedingungen für die Gültigkeit des Gesagten erfüllt sind - das sind, in einem breiteren Sinn, Geltungsansprüche; performative Sprechakte sind eine Untergruppe von Geltungsansprüchen. 
von Themen. Als Organisationen, welche sich dem öffentlichen Kommunizieren verschrieben haben, können Massenmedien in diesem Sinn aus einer systemtheoretischen Perspektive als funktional ausdifferenziertes Teilsystem der Gesellschaft betrachtet werden (Imhof 2006a) ${ }^{5}$.

Massenmediale öffentliche Kommunikation bedeutet in der Regel ein Gefälle zwischen den Massenmedien und den restlichen Teilnehmenden der öffentlichen Kommunikation: Massenmedien kommunizieren mehr in die Öffentlichkeit, als sie selber im Stande sind, aus der Öffentlichkeit aufzunehmen. Die restlichen Teilnehmenden der öffentlichen Kommunikation, bestehend sowohl aus Kollektiv- wie aus Individualakteuren, sind in erster Linie Rezipientinnen und Rezipienten der massenmedialen Kommunikation; nur punktuell treten sie vereinzelt als Sprecherinnen und Sprecher auf. Das bedeutet damit de facto schlicht, dass Massenmedien Publika haben. Eine Kommunikation von Massenmedium zu Publikum bedeutet einen «Fewto-many»-Austausch, bei welchem also der Grossteil der an der öffentlichen Kommunikation Teilnehmenden die Rolle der Rezipienten einnimmt und dabei selber nur selten aktiv kommuniziert. Öffentlichkeit als massenmediale Öffentlichkeit kann darum durchaus als nicht-ideale Öffentlichkeit angesehen werden, weil die aktive Teilnahme am öffentlichen Diskurs nicht ohne Weiteres möglich ist. Der Grund dafür ist, erstens, dass die Teilnahme am massenmedialen öffentlichen Diskurs ein begrenztes Gut ist (die Kommunikationsleistung der Massenmedien ist endlich), und weil, zweitens, Massenmedien eine «Gatekeeper»-Funktion ausüben, also nach bestimmten Logiken einen Bruchteil aller verfügbaren Informationen aus der Gesellschaft selektieren, interpretieren und darstellen. Als eine mögliche Lösung, dennoch geografisch ungebundene öffentliche «Many-to-many»-Kommunikation zu realisieren, wurde schon früh das Internet angesehen (Graham 1998) und dabei teilweise bis hin zu einer «digitalen Agora» hochstilisiert (Rheingold 1993, S. 50), welche einen erstmals wirklich unvermachteten Diskursraum darstellt, in welchem dank dieser erstmals wirklich freien und gleichberechtigten öffentlichen Kommunikation etwa neue Arten politischer Akteure entstehen (Langman 2005). Diese idealistische Lesart des Internets als Ersatz für massenmediale Öffentlichkeit deckt sich indes bisher nicht mit der empirischen Realität, und zwar aus unterschiedlichen Gründen. Individuelle Teilnehmerinnen und Teilnehmer öffentlicher Online-Kommunikation wenden sich faktisch zwar an die Öffentlichkeit, tun dies oftmals aber in einem halbprivaten Kommunikationsmodus (Rasmussen 2009). Das bedeutet, dass eine der Prämissen von Öffentlichkeit als öffentlichem Kommunizieren - der imaginierte Adressat «Öffentlichkeit» - oftmals nicht erfüllt ist. Darüber hinaus gibt es gute Gründe, anzunehmen, dass ein Grossteil der nicht-journalistischen,

$5 \quad$ Ob diese Ausdifferenzierung ein tatsächlicher historischer Prozess war, oder, ob es sich beim Konzept der Ausdifferenzierung eher um eine systemtheoretische Metapher handelt, soll an dieser Stelle nicht weiter problematisiert werden. 
nicht-professionellen politischen Online-Kommunikation Anschlusskommunikation ist (Meraz 2011; Neuberger, Nuernbergk und Rischke 2007; Russell u. a. 2015; Sayre u. a. 2010): Das, was online für Gesprächsstoff sorgt, ist nach wie vor weitgehend das, was die klassischen Medien journalistisch herstellen (und durchaus auch über Online-Kanäle verbreiten). Das bedeutet, dass politische «Laienkommunikation» im Internet keinen Ersatz für klassische journalistische Arbeit in professionellen Medien darstellt, sondern allenfalls eine Ergänzung, denn Medien bilden nach wie vor das «Masterforum» (Ferree 2002, S. 10) öffentlicher Kommunikation.

Wenn also Öffentlichkeit nach wie vor am ehesten als massenmediale Öffentlichkeit verstanden werden kann, worin liegt nun der Sinn einer solchen massenmedialen Öffentlichkeit? Es ist zunächst naheliegend, eine Antwort auf diese Frage aus einer normativ-präskriptiven Perspektive zu suchen; der Sinn von Öffentlichkeit ist das, was Öffentlichkeit leisten soll. Es gibt allerdings keine einheitliche normative Sichtweise darüber, was genau Öffentlichkeit leisten soll. Eine der bekannteren Positionen in dieser Frage ist die Idee von Öffentlichkeit als diskursivem Raum, in welchem sich eine bestimmte Form rationalen Diskurses, «Deliberation», entfalten soll, um damit rationaliter die Qualität der politischen Entscheidungsfindung zu erhöhen (Habermas 2008; Habermas, S. Lennox und F. Lennox 1974). Andere normative Öffentlichkeitskonzepte postulieren andere Kriterien als normativ relevant, etwa in der liberalen Tradition einen Pluralismus bei den Kommunikationsteilnehmenden bzw. bei den in der Öffentlichkeit vorhandenen Meinungen (Dahlgren 2006), oder in der auf Rousseau (1762) zurückgehenden republikanischen Tradition die Forderung nach einer kommunitaristischen Transformation des Individuums zum Citoyen durch die Teilhabe an der Öffentlichkeit als öffentlichem Willen. Diese unterschiedlichen normativen Verständnisse vom Sinn (massenmedialer) Öffentlichkeit, welche ich hier nur mit grösster Oberflächlichkeit erwähne, widersprechen sich ein Stück weit, aber dennoch haben sie alle gleichzeitig Gültigkeit im Sinne normativer Akzeptabilität: Unterschiedliche normative Forderungen an Öffentlichkeit lassen sich rational begründen, und dabei ist keine Position notwendigerweise «richtiger» oder «falscher» als die andere. Damit weist Öffentlichkeit deutliche Eigenschaften eines grundlegend umstrittenen Konzeptes ${ }^{6}$ (Gallie 1955) auf. Diese grundlegende Umstrittenheit hinsichtlich einer normativ-präskriptiven Lesart des Sinnes massenmedialer Öffentlichkeit lässt sich also nicht auflösen. Der Sinn massenmedialer Öffentlichkeit, so meine ich, ergibt sich denn auch nicht aus einer normativ-präskriptiven Perspektive, sondern aus einer funktional-deskriptiven Perspektive: Der Sinn massenmedialer Öffentlichkeit ist nicht etwas, was diese Öffentlichkeit machen soll, sondern etwas, was Öffentlichkeit effektiv leistet.

\footnotetext{
6 «Essentially contested concept» im englischen Original.
} 
Die Idee, Öffentlichkeit im Sinne der Funktionen von Öffentlichkeit zu betrachten ist nicht neu. Das gängige Verständnis von Öffentlichkeitsfunktionen aber, beschrieben etwa bei Peters, Wessler und Habermas (2007, S. 62-65) und Imhof (2008, S. 20-27), ist wiederum ein normativpräskriptives, bei welchem letztlich wieder postuliert wird, was Öffentlichkeit idealiter leisten soll und nicht, was sie empirischerweise effektiv leistet. Es gibt auch einzelne Beiträge, in denen die funktionale Perspektive von Öffentlichkeit ohne normative Forderungen beschrieben wird, etwa bei Luhmann (1996). Eine solche systemtheoretische (Luhmann 1984) Perspektive hat aber den Nachteil, dass der Funktionalismus in der Öffentlichkeitsbeschreibung relativ empiriefern ist und eher den Zweck hat, die systemtheoretische Perspektive an und für sich zu validieren.

Einen Vorschlag, die Funktionen von Öffentlichkeit empirienah zu beschreiben, machen Rauchfleisch und Kovic (2016). In diesem Beitrag unterscheiden wir nach vier generalisierten und in einer quasi-Maslow'schen Manier hierarchischen Funktionen von Öffentlichkeit. Die erste und grundlegende Funktion ist das Stiften kollektiver Identität. Das Herausbilden kollektiver Identität ist spätestens seit dem Konzept der Vergemeinschaftung (M. Weber 2006, S. 328-361) eine in den Sozialwissenschaften stark verbreitete Idee. Die zweite Funktion von Öffentlichkeit ist «Agenda Setting» im Sinne der Rezeptivität der politischen Elite auf öffentliche Kommunikation als Reize ${ }^{7}$. Die dritte Funktion ist Kritik und Kontrolle im Sinne eines «Policy Cycle» (Lasswell 1956). Die vierte Funktion von Öffentlichkeit schliesslich ist Deliberation. Bei der Unterscheidung nach diesen vier Funktionen von Öffentlichkeit is eines unserer Hauptargumente, dass diese Funktionen grundsätzlich universal sind, dass öffentliche Kommunikation also überall grundsätzlich dieselben Funktionen ausübt. Aber, so argumentieren wir weiter, der Grad, in welchem die jeweiligen Funktionen auch wirklich aktualisiert werden, ist abhängig von dem empirischen, strukturell-institutionellen Kontext des jeweiligen politischen Geltungsraumes.

Der politische Geltungsraum, um welchen es in dieser Arbeit geht, ist die Schweiz. Wenn auch die Schweiz dank Proporz-Wahlsystem, ausgeprägtem Föderalismus, direkter Demokratie und Konkordanz einen «Prototypen» für ein Konsensus-System darstellt (Lijphart 2012, S. 245) und damit im internationalen Vergleich aus der Reihe tanzt, ist die Schweiz recht eindeutig eine funktionierende repräsentative Demokratie mit klarer Gewaltenteilung, Rechtsstaatlichkeit, freien Wahlen und ausgeprägten Bürgerrechten (Economist Intelligence Unit 2015). Der Umstand, dass die Schweiz als funktionierende Demokratie angesehen werden kann, hat eine bestimmte Implikation für die Institution der Legislative in der Schweiz. Dadurch, dass das Parlament in der Schweiz unter demokratischen Bedingungen operiert, wird eine der Öffent-

$7 \quad$ Damit ist die eher politikwissenschaftliche Auffassung von Agenda Setting gemeint, welche nicht ganz identisch mit der kommunikationswissenschaftlichen ist. Mehr zum Konzept des Agenda Setting in Unterabschnitt 3.1 . 
lichkeitsfunktionen nach Rauchfleisch und Kovic (2016), die Kontroll- und Kritikfunktion, zu einer notwendigen Bedingung für das Funktionieren des Parlamentes. Diese enge Verwebung des Parlamentes mit dieser spezifischen Funktion von Öffentlichkeit nenne ich die kommunikative Legitimität des Parlamentes, und diese kommunikative Legitimität ist die normative Triebfeder für die vorliegende Arbeit. In Unterunterabschnitt 2.1.4 soll die kommunikative Legitimität des Parlamentes hergeleitet und näher begründet werden. Zunächst aber lege ich im nachfolgenden Unterunterabschnitt 2.1.3 dar, wie genau ich das Handeln der für diese Arbeit relevanten Akteure konzipiere.

\subsubsection{Zweckrationales Handeln und Präferenzen}

Eine jede sozialwissenschaftliche Auseinandersetzung mit der Frage des menschlichen Handelns muss als Ausgangspunkt, so meine ich, mit Prämissen operieren, welche entweder direkt der ontologischen Realität des Menschen entspringen, oder im Mindesten mit diesen kompatibel sind. Sozialwissenschaftliche Konzepte zum menschlichen Handeln, welche in ihren Prämissen - um es in etwas grossen Worten auszudrücken - nicht mit der wahrscheinlichen Rolle des Menschen im Universum vereinbar sind, sind bestenfalls unnütz, schlimmstenfalls irreführend: Wenn wir in unseren Modellen menschlichen Handelns auf Prämissen rekurrieren, welche nicht belegt oder gar widerlegt sind, dann stellen diese Modelle ein Problem dar, denn die Schlussfolgerungen der in diesen Prämissen gründenden Forschung können in beträchtlich falsche Richtungen deuten ${ }^{8}$.

Wenn sich handlungstheoretische Modelle also an der ontologischen Realität des Menschen als Lebewesen orientieren sollen, wie genau sieht diese Realität aus? Was ist - um nochmals die grossen Worte aufzugreifen - die Rolle des Menschen im Universum? Alles, was wir über die Herkunft des Menschen wissen, deutet darauf, dass wir Menschen ebenso wie alles andere bisher bekannte Leben im Universum die Folge evolutionärer Vorgänge, also genetischer Mutation und Selektion, sind. Wir Menschen gehören zu der Domäne der Eukaryoten, zu dem Reich der Tiere, zu dem Genus Homo, zu der Spezies Homo Sapiens, und wir haben unseren evolutionären Ursprung mit hoher Wahrscheinlichkeit in Ostafrika (Horai u. a. 1995; Liu u. a. 2006; C. B. Stringer und Andrews 1988; C. Stringer 2003). Das bedeutet nicht, dass sozialwissenschaftliche

$8 \quad$ Mit falsch meine ich nicht falsch hinsichtlich der formalen Logik, sondern hinsichtlich der informalen Logik. So ist etwa die Schlussfolgerung Wenn die Sonne aufgeht, fährt Helios im Sonnenwagen über den Himmel. Die Sonne geht auf. Helios fährt im Sonnenwagen über den Himmel. formallogisch wahr. Damit ist aber die inhaltliche Wahrheit der Schlussfolgerung, also die Frage, ob es wirklich einen Helios gibt, der im Sonnenwagen über den Himmel fährt, nicht beantwortet. Genau dies meine ich mit der Forderung, die Prämissen sozialwissenschaftlicher Forschung müssten informal, also evidenzbasiert wahr sein: Forschung, welche auf falschen Prämissen aufbaut, kann durchaus formallogisch wahr sein. 
Handlungstheorie unmittelbar auf evolutionsbiologische Vorgänge rekurrieren soll. Es gibt Versuche, sozialwissenschaftliche Forschung direkt an evolutionsbiologische Vorgänge zu knüpfen, allen voran in der Forschungstradition der sogenannten evolutionären Psychologie, innerhalb derer psychologische Vorgänge als evolutionäre Adaptionen verstanden werden (Buss 2008, S. 69). Wenn auch die Verfechter der evolutionären Psychologie meinen, prüfund falsifizierbare Hypothesen zu untersuchen (Holcomb III 1996; Sell u.a. 2006), ist das, was im Kontext evolutionärer Psychologie untersucht wird, letztlich nicht Evolution - ob bestimmte Verhaltens- und Handlungsmuster im Laufe der menschlichen Entwicklung als funktionaler Nutzen evolutionär selektiert wurden, kann rein logischerweise nicht dadurch untersucht werden, dass wir beobachten, ob diese Verhaltens- und Handlungsmuster heute bei Menschen vorhanden sind. In diesem Sinne demonstriert evolutionäre Psychologie, dass Sozialwissenschaft nicht direkt an die evolutionsbiologische Natur des Menschen anknüpfen kann, da sich damit bestenfalls abduktive Vermutungen formulieren lassen. Eher geht es darum, dass Sozialwissenschaft mit der evolutionsbiologischen Natur des Menschen kompatibel sein soll. Diese Kompatibilität der Sozialwissenschaft mit der evolutionsbiologischen Natur des Menschen kann in einem negativen und in einem positiven Sinn gegeben sein.

Die negative, oder ausschliessende, Kompatibilität sozialwissenschaftlicher Handlungstheorien mit der evolutionsbiologischen Natur des Menschen besteht darin, nicht mit theoretischen Prämissen zu operieren, welche in direktem Widerspruch zu erhärtetem evolutionsbiologischen Wissen über den Menschen stehen. Bei diesen Prämissen handelt es sich in erster Linie um ideengeschichtlich vorwissenschaftliche Annahmen über die Natur des Menschen, etwa teleologische Vorstellungen aus der antiken Philosophie, religiös geprägte Vorstellungen u.a. im Kontext der abrahamitischen Religionen, Rassentheorien ${ }^{9}$, Dualismus ${ }^{10}$, und so fort.

Die positive Kompatibilität sozialwissenschaftlicher Handlungstheorien mit der evolutionsbiologischen Natur des Menschen besteht darin, solche theoretischen Prämissen zu verwenden, welche angesichts der menschlichen Biologie Sinn machen. Das bedeutet in erster Linie, dass als Ursprung des menschlichen Handelns das menschliche Hirn verstanden wird, und, dass folglich die Art und Weise, wie das menschliche Hirn operiert, in Einklang ist mit der Art und Weise, wie menschliches Handeln sozialwissenschaftlich gedacht wird. Diese Idee der positiven Kompatibilität sozialwissenschaftlicher Handlungstheorie mit der evolutionsbiologischen Natur des Menschen beschreibt Searle (2010, S. 4) wie folgt:

9 «Rasse» ist ein soziales, kein biologisches Konzept (A. Smedley und B. D. Smedley 2005; Templeton 2013).

10 Dualismus meint die Vorstellung, dass das menschliche Bewusstsein getrennt ist vom physischen menschlichen Körper (Feyerabend 1963). 
Our mental life depends on the basic facts. Both conscious and unconscious mental phenomena are caused by neurobiological processes in the brain and are realized in the brain $[\ldots]$.

This is the basic requirement of our enterprise: the account must be consistent with the basic facts and show how the nonbasic facts are dependent on and derived from the basic facts. [Kursiv im Original]

Die von Searle angesprochenen «nonbasic facts» meinen die Umstände der sozialen Realität: Dass Menschen in sozialen Gruppen handeln, wie sie handeln, ist nicht eine unmittelbar offensichtliche Konsequenz der grundlegenden biologisch-physikalischen Realität des Menschen als Produkt der Evolution, aber menschliches Handeln muss logischerweise in dieser grundlegenden Realität gründen, so wir sie akzeptieren. Searle fokussiert in seiner Analyse auf einen bestimmten Handlungstyp, die in Unterunterabschnitt 2.1.2 erwähnten Sprechhandlungen oder Sprechakte.

Für die vorliegende Arbeit interessiere ich mich nicht für den Handlungstyp der Sprechhandlungen, sondern für den Handlungstyp des zweckrationalen Handelns (M. Weber 2006, S. 32): Ich verstehe Menschen als Akteure, welche Ziele haben und auf eine solche Art handeln, dass sie ihre Ziele verwirklichen. Durch den Umstand, dass Akteure Ziele haben, haben sie automatisch Präferenzen. Akteure können unterschiedliche Ziele haben, und unter den möglichen unterschiedlichen Zielen bevorzugen sie einige Ziele mehr als andere (H. A. Simon 1955; Tversky und Kahneman 1986). Das bedeutet, dass zweckrational handelnde Akteure Ziele verfolgen und dabei wissen, welche Ziele sie eher verwirklichen wollen und welche weniger. Zweckrationalität wird bisweilen auch als strategisches Handeln (Habermas 1981, S. 131) oder als instrumentelle Rationalität (T. Kelly 2003) beschrieben.

Ist das Modell von Akteuren als zweckrational handelnden Akteuren eines, welches in einem positiven Sinn kompatibel mit den «basic facts», also mit der biologischen Realität des Menschen, ist? Ich meine ja, aber nicht selbstverständlicherweise, denn es kommt darauf an, mit was für Vorstellungen ein Modell zweckrational handelnder Akteure verbunden ist. Falls zweckrational handelnde Akteure Akteure sein sollen, welche entweder perfekt informiert sind, oder mindestens ihren Informationsstand perfekt einschätzen können, und sie diese perfekte Informiertheit in effiziente und perfekt zielorientierte Entscheidungsfindung einbringen können, dann ist das handlungstheoretische Modell nicht kompatibel mit den «basic facts». Das menschliche Hirn als Ursprung menschlichen Handelns ist nicht auf Perfektion ausgelegt, sondern eher auf ein «gut genug». Konkret findet menschliche Kognition in zwei tendenziell unterschiedlichen Modi statt, einem «schnellen» und einem «langsamen» (Evans 2003; 
Frankish 2010; Neys 2006). Den Grossteil unserer Denkarbeit erledigen wir in dem schnellen, automatisierten Modus. In diesem Denkmodus handeln wir nicht sinngeleitet, sondern sind näher an blossem Verhalten, da wir uns in diesem Modus mittels Routine und Heuristiken durch den Alltag manœvrieren. Im Gegensatz dazu meint der langsame Denkmodus das bewusste, reflektierte und explizite Analysieren und Überlegen, u.a. in Form von Metakognition, dem Denken über das eigene Denken (Amsel u. a. 2008; Fletcher und Carruthers 2012).

Ist das Modell des zweckrational handelnden Akteurs, welcher auf die Verwirklichung von Präferenzen hinarbeitet, angesichts der teils automatisierten und unreflektierten kognitiven Arbeit des menschlichen Gehirns haltbar? Ich denke, dass es das ist, aber in einer Form, in welcher die handlungsleitenden Motivstrukturen von Akteuren getrennt sind von der Frage der Realisierung der mit den Handlungen der Akteure verbundenen Präferenzen. Akteure können zielorientiert handeln und sie können dabei Präferenzordnungen haben - das bedeutet aber weder, dass die Präferenzen in irgendeiner Form umfassend oder wohlüberlegt sind, noch, dass die Akteure optimal auf das Erreichen ihrer Präferenzen hinarbeiten. Das Rationale an zweckrationalem Handeln besteht in dieser Lesart also nicht in der perfekten, aber realitätsfernen perfekten Denkleistung von Akteuren, sondern in der möglichen Motivstruktur der Akteure.

Für die vorliegende Arbeit unterstelle ich die Motivstruktur des zweckrationalen Handelns drei Gruppen von Akteuren und in drei Handlunkgskontexten: Bürgerinnen und Bürgern beim Wählen; Parlamentarierinnen und Parlamentariern beim Legiferieren; Journalistinnen und Journalisten beim Herstellen öffentlicher Kommunikation zum Parlament. Diese drei Akteursgruppen und ihre jeweiligen Präferenzen stehen in einem normativ aufgeladenen Verhältnis zueinander. In was für einem, erkläre ich zunächst in Unterunterabschnitt 2.1.4 für die Beziehung zwischen Bürgerinnen und Bürgern, um später in Unterunterabschnitt 2.2.2 zu erläutern, welche Rolle zusätzlich die Präferenzen von Journalistinnen und Journalisten spielen.

\subsubsection{Die kommunikative Legitimität des Parlamentes}

Wenn Politik als das Herstellen und Durchsetzen allgemein verbindlicher Regeln verstanden wird, ist demokratische Politik eine solche Politik, unter welcher jene, die sich Gesetzen fügen, gleichzeitig die Autorinnen und Autoren dieser Gesetze sind. Das ist, sehr kompakt zusammengefasst, das Prinzip der Volkssouveränität. Nun ist es aber nicht problemlos möglich, das Prinzip der Volkssouveränität im politischen Alltag auch tatsächlich umzusetzen. Dieses Argument ist nicht neu. So ist etwa bereits Rousseau (1762, S. 93) in seiner Analyse der Regierungsform der Demokratie berühmterweise zu diesem Schluss gekommen: 
S’il y avait un peuple de dieux, il se gouvernerait démocratiquement. Un gouvernement si parfait ne convient pas à des hommes.

Hiermit spricht Rousseau den Umstand an, dass es praktisch nicht möglich ist, dass alle Bürgerinnen und Bürger zu jedem Zeitpunkt ihre Souveränität in Form aktiven politischen Handelns ausüben - nur schon, weil Ressourcen wie Zeit begrenzt sind ${ }^{11}$. Dieser Umstand wurde also schon früh erkannt, und früh folgte auch die Schlussfolgerung aus diesem Umstand, so etwa prominent bei Locke (1689): Wenn Bürgerinnen und Bürger ihre Souveränität nicht permanent in Form aktiven politischen Handelns ausüben können, ist eine Arrangement von Institutionen nötig, welches im Auftrag des Volkes zeitlich befristet die Aufgaben des Politischen wahrnimmt. Repräsentative Demokratie ist also der Versuch, das Konzept der Volkssouveränität empirisch umzusetzen.

Das «Repräsentative» an repräsentativer Demokratie kommt nicht automatisch und selbstverständlich zustande, wenn versucht wird, Volkssouveränität in demokratischem Sinne empirisch umzusetzen. Für uns - als «wir» seien in etablierten demokratischen Gesellschaften sozialisierte Menschen angesprochen - mag es zwar selbstverständlich sein, dass wir über Parlamente verfügen, welche wir mit über Wahlen ermittelten Volksvertreterinnen und Volksvertretern besetzen. Im Grunde aber liesse sich Volkssouveränität auch über den einfacheren Mechanismus der Auslosung bestimmen (Manin 1997, S. 42-93), indem aus der Grundgesamtheit aller Bürgerinnen und Bürger periodisch Personen ausgelost werden, welche auf Zeit das Amt der Gesetzgebung ausüben. Der Anspruch, dass sich das politische Personal des Parlamentes aus der Grundgesamtheit aller Bürgerinnen und Bürger speisen soll, ist eine notwendige Bedingung auch für Volkssouveränität als repräsentative Demokratie, aber es ist noch keine hinreichende Bedingung. Repräsentativität speist sich nämlich zusätzlich aus der Art und Weise, wie das Parlament besetzt wird: Erst dadurch, dass die Besetzung des Parlamentes durch einen aktiven, nicht-zufälligen und informierten Entscheidungsmoment seitens des Souveräns, sprich, durch die oben erwähnten Wahlen, geschieht, kann davon ausgegangen werden, dass das parlamentarische Kollektiv den Willen des Kollektivs der Bürgerinnen und Bürger darstellt.

Volkssouveränität als repräsentative Demokratie bedeutet also, dass nicht nur die blosse Eigenschaft der Legislative, dass sie sich personnell aus der Grundgesamtheit der Bürgerinnen und Bürger speist, von Interesse ist. Es geht in einem zweiten Schritt ganz wesentlich auch um die Art und Weise, wie genau eine Auswahl an Bürgerinnen und Bürgern bestimmt wird, welche

$11 \quad$ Wenn Rousseau von Demokratie spricht, meint er eine radikal-direkte Demokratie; also eine, bei der effektiv alle Bürgerinnen und Bürger an der Herstellung und Durchsetzung aller allgemeinverbindlicher Regeln mitwirken. 
in der Legislative Einsitz findet - bei repräsentativer Demokratie geht es also nicht bloss um das Was, sondern auch ebenso zentral um das Wie. Das gängige Wie in funktionierenden repräsentativen Demokratien ${ }^{12}$, so auch in der Schweiz, sind Wahlen. Doch es ist nicht der blosse Akt des Wählens, welcher einen aktiven, nicht-zufälligen und informierten Entscheidungsmoment darstellt. Eine Wahl kann prinzipiell nämlich bedeuten, dass mehr oder weniger willkürlich Kreuze auf dem Wahlformular gesetzt werden. Rein formal mag das eine korrekte und gültige Wahl sein, aber in einem demokratischen Kontext, in welchem das Wie wichtig ist, ist eine Grundvoraussetzung, dass die Wahl sinngeleitet ist. Das bedeutet, dass jene, die wählen, dies in irgendeiner Art überlegt machen: Die Wählerschaft hat wie auch immer geartete Präferenzen und sie bringt diese bei der Wahl zum Ausdruck. Repräsentative Demokratie beruht also nicht bloss auf formalen Institutionen, sondern ebenso auf der Informiertheit und Reflektiertheit der Bürgerinnen und Bürger (Milner 2002, S. 11-50). Diese Reflektiertheit und Informiertheit als notwendige Bedingung für repräsentative Demokratie meint nicht, dass nur umfassend informierte und stets engagierte Bürgerinnen und Bürger, wie sie etwa de Tocqueville idealisierte (Goldstein 1964), Demokratie ermöglichen. Eher ist damit eine qualitative Minimalanforderung gemeint, welche aus zwei Bedingungen besteht:

- Das Ausmass der politischen Präferenzen der Bürgerinnen und Bürger ist grösser als Null.

- Der Grad der Informiertheit über das politische Geschehen der Bürgerinnen und Bürger is grösser als Null.

Die zweite Bedingung ist aus dem offensichtlichen Grund notwendig, dass nur eine informierte Entscheidungsfindung bedeuten kann, dass Bürgerinnen und Bürger ihre Präferenzen tatsächlich sinngeleitet umsetzen. Ein banales Beispiel: Wenn eine Bürgerin möchte, dass das Parlament die Landesverteidigung, also die Armee, stärker fördert (d.h., diese Bürgerin hat eine konkrete Präferenz im Spektrum aller möglichen Entscheidungen, welche das Parlament treffen kann), dann muss diese Bürgerin einen Grad der Informiertheit über die politischen Parteien wie auch die individuellen Kandidierenden haben, welcher grösser als Null ist. Nur dann ist es nämlich möglich, dass der Wunsch dieser Kandidatin zumindest potenziell mit ihrer Wahlentscheidung sinngeleiteterweise übereinstimmt. Diese Übereinstimmung ist nur potenziell, weil der Grad und die Qualität der Informiertheit der betroffenen Bürgerin sowie die Qualität der Informationsverarbeitung der Bürgerin variieren können. Die Wahlentscheidung

$12 \quad$ Praktisch alle Staaten auf der Welt haben eine Legislative, aber nur eine Minderheit der Staaten mit einer Legislative kann als funktionierende Demokratie angesehen werden (Power und Shoot 2013). 
ist aber dann und nur dann sinngeleitet, wenn der Grad der Informiertheit grösser als Null ist. Wenn der Grad der Informiertheit dieser fiktiven Bürgerin gleich Null ist, dann wird ihre Wahlentscheidung zu einer sinnentleerten Lotterie.

Repräsentative Demokratie, in welcher sich die Bürgerinnen und Bürger auf eine solche sinngeleitete Art und Weise in politischer Entscheidungsfindung üben, kann als prozeduralisierte Volkssouveränität (Habermas 1994, 1997) beschrieben werden. Wenn auch die aktive politische Entscheidungsfindung auf punktuelle Momente wie etwa Wahlen beschränkt ist, sind die Bürgerinnen und Bürger stetig in den Fluss des Politischen eingebunden, indem sie, erstens, Präferenzen haben, und indem, zweitens, das Mass, in welchem die Bürgerinnen und Bürger passive Teilnehmende des politischen Diskurses sind (d.h., über Informationen des politischen Geschehens verfügen), grösser als Null ist.

Dieses generelle Prinzip der doppelten Sinnhaftigkeit repräsentativer Demokratie - Präferenzen gekoppelt mit Informiertheit - äussert sich besonders stark im Verhältnis der Bürgerinnen und Bürger zum Parlament. Doppelte Sinnhaftigkeit ist zweifellos auch in anderen Aspekten der politischen Entscheidungsfindung relevant, etwa in Sachabstimmungen wie Referenden und Initiativen im Schweizer Kontext, aber das Prinzip äussert sich am klarsten bei der Legislative, der Kerninstitution repräsentativer Demokratie. Das Parlament steht in einer bestimmten und beständigen Beziehung zu den Bürgerinnen und Bürgern. Wahlen finden zwar in der Regel nur in bestimmten Zeitabständen statt; in der Schweiz dauert eine Legislatur auf Bundesebene vier Jahre. Das hat aber nicht zur Folge, dass die gewählten Parlamentarierinnen und Parlamentarier für die gesamte Amtszeit komplett losgelöst von den Bürgerinnen und Bürgern operieren, auch wenn sie, formal gesehen, für die Dauer ihrer Amtszeit nicht mehr aktiv abgewählt werden können. Das Parlament steht in einem steten Rechenschaftsverhältnis zu den Bürgerinnen und Bürgern (Przeworski, S. C. Stokes und Manin 1999): Die Wahl in das Parlament bedeutet einen Auftrag seitens der Wählerschaft, und dadurch, dass die Wählerschaft diesen Auftrag ausspricht, werden die in das Parlament Gewählten gegenüber den Bürgerinnen und Bürgern rechenschaftspflichtig. Dieses Rechenschaftsverhältnis ist nicht auf eine Art formalisiert, wie das beispielsweise in privatwirtschaftlichen Kontexten üblich ist: Es gibt keinen formalen Vertrag, den die Wählenden und die Gewählten unterschreiben; es gibt keine formalen Verpflichtungen, welche die Gewählten eingehen; es gibt keine formalen Mechanismen der Evaluation; es gibt, bis auf die Verweigerung der Wiederwahl, keine formalen Sanktionsmöglichkeiten für die Wählenden, falls sie mit der Arbeit der Gewählten nicht einverstanden sind; es existieren keinerlei juristische Rekursmöglichkeiten für «Vertragsbruch», weil es keinen Vertrag gibt. Das Rechenschaftsverhältnis des Parlamentes zu den Bürgerinnen und Bürgern ist recht eindeutig 
also kein vertraglich-legales Problem. Es handelt sich eher um ein informelles Problem, welches oft in politischen Konstellationen zu beobachten ist: Ein sogenanntes Prinzipal-Agent-Problem.

Eine Prinzipal-Agent-Beziehung zeichnet sich dadurch aus, dass ein Auftraggeber (Prinzipal) und Auftragnehmer (Agent) in einer zwangsläufig suboptimalen Beziehung stehen. Der Agent würde idealerweise die Präferenzen des Prinzipals zu 100\% umsetzen, aber es gibt einen wesentlichen Grund, warum eine perfekte Umsetzung unwahrscheinlich ist: Die Präferenzen des Prinzipals stimmen sehr wahrscheinlich nie perfekt mit den Präferenzen des Agenten überein, und infolge dieser Inkongruenz von Präferenzen unterlässt der Agent Handlungen zugunsten des Prinzipals, oder handelt aktiv zuungunsten des Prinzipals (Kiewiet und McCubbins 1991, S. 22-38; Grossman und Hart 1983; Laffont und Martimort 2002, S. 145-184; Shavell 1979). Die Ursache des Prinzipal-Agent-Problems ist Informationsasymmetrie zwischen dem Prinzipal und dem Agenten (G. J. Miller 2005; Spremann 1987). Der Prinzipal weiss, erstens, nicht perfekt, welche Präferenzen der Agent wirklich hegt. Der Prinzipal weiss, zweitens, nicht perfekt, wie der Agent effektiv handelt. Diese Informationsasymmetrie kommt im Wesentlichen dadurch zustande, dass der Prinzipal nicht perfekt beobachten kann, was der Agent macht (Hölmstrom 1979).

Das Verhältnis der Bürgerinnen und Bürger zum Parlament ist eine fast prototypische politische Prinzipal-Agent-Konstellation (Bergman, Müller und Strøm 2000; P. Mitchell 2000; Strøm 2000). Die Wählerschaft sind die Prinzipale und die von den jeweiligen Gruppen von Wählenden ins Parlament Gewählten sind die Agenten. Weiter oben argumentiere ich, dass prozeduralisierte Volkssouveränität als doppelte Sinnhaftigkeit der Wahlen bedeuten muss, dass Bürgerinnen und Bürger auf eine solche Art sinngeleitet wählen, dass sie ihre Präferenzen in ihrem Wahlakt abgebildet sehen möchten ${ }^{13}$. Die Personen, welche in das Parlament gewählt werden, sind keine präferenzlosen Automata, welche perfekt ihren Auftrag erfüllen, sondern sie verfügen über eigene Präferenzen, welche in einem bestimmten Masse mit den Präferenzen ihrer Wählerschaft übereinstimmen. Das Verhältnis der Wählerschaft als Prinzipal und den Gewählten als Agenten ist geprägt von Informationsasymmetrien. Auch unter fiktiven optimalen Bedinungen ist es empirisch unmöglich, dass die Wählerschaft über dieselben Informationen wie die Parlamentarierinnen und Parlamentarier verfügt. Ein banales Beispiel: Kommissionssitzungen des National- und des Ständerates unterliegen dem Amtsgeheimnis; vertrauliche Informationen aus den Sitzungen dürfen nicht öffentlich werden. Wenn auch Informationsasymmetrie in der Beziehung zwischen Bürgerinnen und Bürgern als der Wählerschaft und Oder, im «Rational Choice»-Jargon ausgedrückt: Die Wählerschaft will bei der Wahl ihren Nutzen («Utility»)
maximieren (Shepsle 1972). 
den gewählten Parlamentarierinnen und Parlamentariern unvermeidbar ist, kann das Mass an Informationsasymmetrie variieren - und zwar, weil das Mass an öffentlicher Beobachtbarkeit des Parlamentes variieren kann.

Hierin offenbart sich nun die kommunikative Legitimität des Parlamentes. Wenn die Beziehung zwischen Bürgerinnen und Bürgern und dem Parlament als eine zwischen Prinzipal und Agent zu verstehen ist, dann kann diese Beziehung dann und nur dann Bestand haben, wenn der Prinzipal grundsätzlich die Möglichkeit hat, das Handeln des Agenten zu beobachten. Beobachtbarkeit des Agenten als Beobachtbarkeit des Parlamentes bedeutet, dass das Handeln des Parlamentes Gegenstand öffentlicher Kommunikation ist. Erst dadurch, dass das Parlament Gegenstand öffentlicher Kommunikation ist, erhält das Parlament Legitimität im Sinne prozeduralisierter Volkssouveränität: Die Bürgerinnen und Bürger als Prinzipale erhalten Informationen über das Handeln des Parlamenten als Agent, und diese Information bedeutet, dass die Entscheidungsfindung der Bürgerinnen und Bürger das Kriterium der doppelten Sinnhaftigkeit erfüllt. Im Sinne der Öffentlichkeitsfunktionen nach Rauchfleisch und Kovic (2016) handelt es sich bei der kommunikativen Legitimität des Parlamentes um die Kritik- und Kontrollfunktion, denn die Beobachtbarkeit des Parlamentes ermöglicht es den Bürgerinnen und Bürgern als Prinzipal, das Handeln des Parlamentes als Agent zu kontrollieren und das eigene Handeln danach auszurichten, etwa in Form von Sanktionen (z.B. der Abwahl bzw. Nicht-Wiederwahl).

Die Notwendigkeit der öffentlichen Sichtbarkeit des Parlamentes wurde bereits verschiedentlich vermerkt, etwa bei Ettinger (2005), Marschall (2002, 2003) und Oberreuter (1997). Diese bisherigen Begründungen sind in ihrer Argumentation durchaus plausibel, aber, so meine ich, zu wenig radikal. Das Verhältnis des Parlamentes zur Öffentlichkeit wird in der Regel nämlich konzipiert als das Verhältnis einer politischen Institution, welche an und für sich abseits der Öffentlichkeit exisitiert, zur Öffentlichkeit. In Tat und Wahrheit, so das oben hergeleitete Argument, ist das Parlament aber nicht getrennt von der Öffentlichkeit, sondern fundamental mit ihr verwoben: Erst dadurch, dass das parlamentarische Handeln Gegenstand öffentlicher Kommunikation ist, dass also das Parlament öffentlich beobachtbar ist, kommt das Prinzip prozeduralisierter Volkssouveränität zur Entfaltung. Erst ein solches Parlament, welches Gegenstand öffentlicher Kommunikation ist, kann also als wirklich repräsentatives Parlament gelten - diese Eigenschaft des Parlamentes macht dessen kommunikative Legitimität aus. 


\subsection{Problemaufriss und Forschungsfragen}

\subsubsection{Medienwandel, Medienkrise und das Parlament}

Es ist an und für sich trivial, zu bemerken, dass sich Medien gegenwärtig in einem Wandel befinden, denn Medien als soziale Gebilde befinden sich grundsätzlich in einem Zustand steter Veränderung. Der Wandel, welchen Medien seit ca. Anfang der 2000er Jahre erleben, ist aber durch umbruchartige Veränderungen gekennzeichnet, welche weit mehr als blosse inkrementelle Routineveränderungen sind. Es handelt sich nämlich um Probleme, welche mit den bisher vorhandenen Problemlösungsstrategien nicht bewältigt werden können. Zudem haben die umbruchartigen Veränderungen Auswirkungen nicht nur auf das Mediensystem, sondern auch auf die weiteren gesellschaftlichen Teilsysteme. Die gegenwärtigen rasanten Veränderungen der Medien tragen somit Züge einer Krise (Seeger, Sellnow und Ulmer 2012) wir sehen uns folglich also mit einer Medienkrise (Meier 2012; Puppis, Künzler und Jarren 2012) konfrontiert.

Einer der Treiber der Medienkrise ist die Krise der finanzierungsmodelle der Medien (Starr 2012), welche aus der Unvereinbarkeit alter Geschäftsmodelle mit den neuen Dynamiken der Online-Kommunikation resultiert (Siles und Boczkowski 2012). Diese Finanzierungskrise lässt sich deutlich an der Entwicklung der Werbeeinnahmen bei Schweizer Zeitungen ablesen, wie sie in Abbildung 1 zusammengefasst ist. Die Werbeeinnahmen bei Zeitungen hatten ihren Höhepunkt Ende der 1980er Jahre und sie verlaufen grundsätzlich zyklisch. Dennoch ist seit dem zweithöchsten Jahresergebnis im Jahr 2000 ein deutlicher Abwärtstrend zu beobachten. Für denselben Zeitraum ist ein Wachstum des Werbevolumens beim Fernsehen zu beobachten, doch ist dieses Wachstum vor allem durch das Wachstum der Schweizer Werbefenster bei ausländischen Fernsehsendern bedingt. Die Volumina für Online-Werbung sind zwar im Wachsen begriffen, sie vermögen allerdings nicht den Rückgang bei den Zeitungen zu kompensieren, zumal nur die Kategorie Display ein eigentliches Online-Äquivalent von Werbung im Print ist. Suchmaschinenwerbung bewirtschaften die jeweiligen Unternehmen, welche die Suchmaschinen betreiben. Online-Rubriken und Online-Verzeichnisse sind zwar Geschäftsbereiche, in denen auch Medienhäuser aktiv sind, aber typischerweise handelt es sich dabei um Diversifikationsstrategien in Form des Aufkaufens bestehender Anbieter. Es ist deutlich, dass Online-Werbung den Wegfall klassischer Printwerbung nicht ohne Weiteres ersetzen wird. Dieser Befund trifft nicht nur für die Schweiz zu, sondern ebenso für den ungleich grösseren Markt der USA (A. Mitchell 2015; Waterman und Ji 2012). Da die Zahlungsbereitschaft für journalistische Inhalte im Internet gering ist (Chiou und Tucker 2013; J. E. Cook und Attari 
Abbildung 1: Entwicklung der Werbeeinnahmen unterschiedlicher Mediengattungen in der Schweiz, 1982 bis 2013 (inflationsbereinigt).

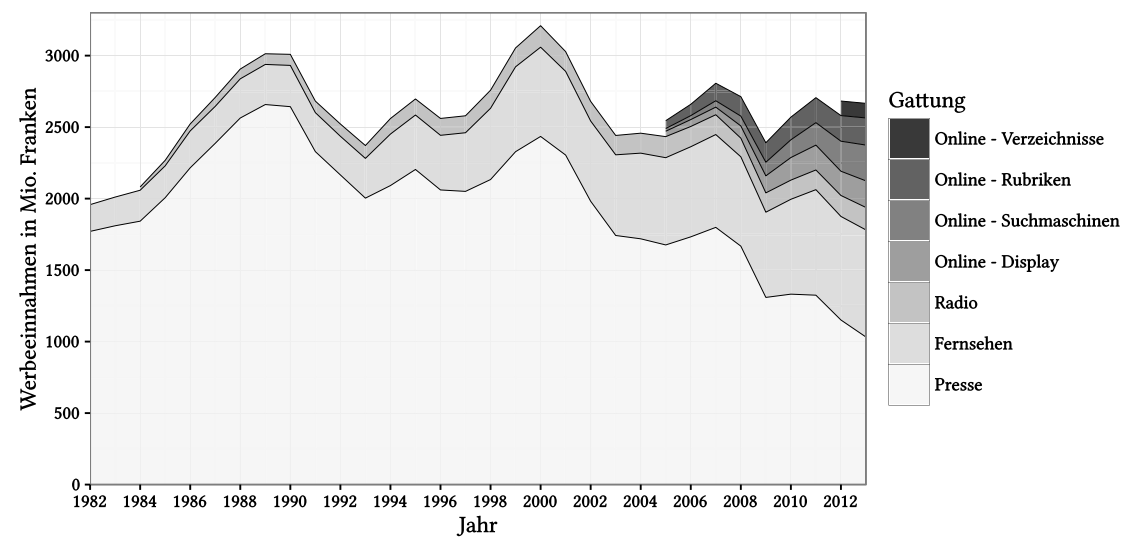

Quelle: Kovic und Sele (2014).

2012; Myllylahti 2014), verschärft sich die Medienkrise. Ein Schweizer Spezifikum befeuert diese Dynamik zusätzlich: Die sogenannten Pendlerzeitungen. Wie in Abbildung 2 dargestellt ist, haben Pendlerzeitungen im Vergleich zu anderen Zeitungstypen zwischen 2003 und 2008 massiv an Auflagen zugelegt und bewegen sich seitdem auf einem stabilen Niveau. Vom Geschäftsmodell her ähneln Pendlerzeitungen Anzeigern, den «klassischen» Gratiszeitungen. Allerdings sind Anzeiger in aller Regel Lokalzeitungen, während Pendlerzeitungen die jeweiligen Sprachregionen abdecken und ein maximal grosses Publikum ansprechen; u.a. dadurch, dass sie an Knotenpunkten des öffentlichen Verkehrs und z.T. innerhalb der Fahrzeuge des öffentlichen Verkehrs aufliegen (darum der Begriff «Pendlerzeitung»).

Die Medienkrise als Medienfinanzierungskrise bedeutet, auf den ersten Blick vielleicht paradoxerweise, nicht, dass Medienhäuser pauschal schlicht relativ kleiner werden, da sich relativ weniger Umsatz über Werbung und Verkauf generieren lässt. Das Gegenteil ist ein Stück weit der Fall, da in der Schweiz deutliche Trends hin zu Medienkonzentration zu beobachten sind (Bonfadelli, Schwarb u. a. 2005; Kamber und Imhof 2011). Mit der unternehmerischen Konzentration geht auch eine publizistische einher, denn nebst der Anzahl der Verlagshäuser sinkt auch die Anzahl der Zeitungstitel (Leonarz 2015, S. 21). Die Medienkonzentration ist am deutlichsten nicht auf sprachregionaler, sondern auf regionaler Ebene (Leonarz u. a. 2011); 
Abbildung 2: Relative Entwicklung der Auflagezahlen unterschiedlicher Zeitungstypen in der Schweiz, 2003 bis 2014 .

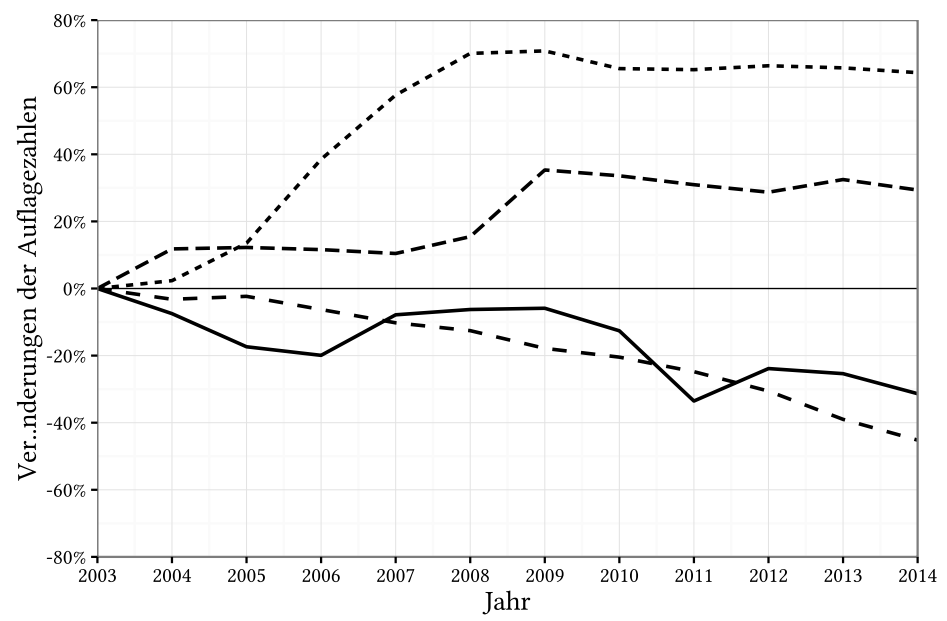

Zeitungstyp $\longrightarrow$ Gratiszeitungen - - - Pendlerzeitungen $\square$ Sonntagszeitungen - - Tageszeitungen (Auswahl)

Quelle: Kovic und Sele (2014). Die Auswahl der Tageszeitungen besteht aus folgenden Titeln: TagesAnzeiger, Neue Zürcher Zeitung, Blick, Le Temps, Le Matin, 24 Heures, Corriere del Ticino.

gegenwärtig verfügen 21 der 26 Kantone nur über eine oder gar keine publizistisch und unternehmerisch eigenständige Tageszeitung (Meier 2015, S. 59; Meier u. a. 2014, S. 59).

Die Medienkrise äussert sich nicht nur in der Finanzierungskrise und dem Trend hin zu Medienkonzentration. Die journalistische Arbeitspraxis hat in den letzten Jahren ebenfalls grosse Veränderungen erlebt (Puppis, Schönhagen u. a. 2014): Das journalistische Personal empfindet eine Verschlechterung der Arbeitsbedingungen infolge steigenden ökonomischen Druckes und beklagt, dass mehr Inhalt mit weniger Ressourcen hergestellt werden muss. Wenn auch Akteure aus dem Medienmanagement davon ausgehen, dass die (kriseninduzierten) Veränderungen im journalistischen Arbeiten die Qualität der Inhalte verbessern (Hofstetter und Schönhagen 2014), dürfte der tatsächliche Effekt der Folgen der Medienkrise auf die Medieninhalte differenzierter ausfallen. Wenn auch «Qualität» ein strittiger Begriff ist, gibt es empirische Indizien dafür, dass sich die Abnahme verfügbarer Ressourcen für das journalistische Arbeiten bei im Mindesten nicht abnehmender Arbeitslast auf inhaltlicher Ebene äussert, in Betreff sowohl der Themenwahl als auch der Themenbearbeitung (fög-Forschungsbereich Öffentlichkeit und 
Gesellschaft 2014, S. 27-50). Das ist nicht zuletzt insofern plausibel, als im Zuge der Medienkrise neue journalistische Formate entstanden sind, welche den Fokus auf das Maximieren von Werbeeinnahmen und das Minimieren der journalistischer Arbeitsleistung legen, etwa sogenannte «Click Bait»-Formate (Blom und Hansen 2015), oder auch das journalismusethisch heikle «Native Advertising» (Carlson 2014).

Der Umstand, dass sich die Schweiz seit ca. den frühen 2000er Jahren in einer Medienkrise befindet, scheint, im Grossen und Ganzen, recht offensichtlich. Das Konzept, oder die Diagnose, dieser Medienkrise ist dabei zunächst nur deskriptiver Natur. Die Beobachtung, dass sich Medien in einer Krise befinden, ist eo ipso noch keine normative Wertung. Der alleinige Umstand nämlich, dass der status quo eines Systems gefährdet ist, ist normativ gehaltlos. Es sind erst die konkreten Folgen einer Krise, welche eine normative Wertung erlauben. Im Falle der Medienkrise wurde schon früh befürchtet, dass die unterschiedlichen Facetten der Medienkrise ein normatives Problem für Demokratie darstellen 5-53 (Baker 2006, S. 5-53; Barnett 2002; McChesney 2003). Auch die vorliegende Arbeit gründet in einer normativen Problemstellung, welche allerdings keinen globalen Charakter hat (es geht nicht grundsätzlich um «die Demokratie»), sondern einen spezifischen. Die kommunikative Legitimität des Parlamentes ist dessen Eigenschaft, dass es demokratische Legitimität im Sinne prozeduralisierter Volkssouveränität dann und nur dann inne hat, wenn die Bürgerinnen und Bürger als Prinzipale die Möglichkeit haben, das Parlament als Agenten öffentlich zu beobachten. Diese öffentliche Sichtbarkeit des Parlamentes wird im Wesentlichen durch die Medien bereitgestellt. Wenn die Medien sich nun in einer Medienkrise befinden und diese Medienkrise einen Einfluss auf die von Medien hergestellte öffentliche Kommunikation hat, dann drängt sich zwangsläufig die Frage auf, wie es um die öffentliche Sichtbarkeit des Parlamentes im Zuge der Medienkrise steht.

\subsubsection{Die Schnittmengen der Politiklogiken und der Medienlogiken}

In der theoretischen Skizze der kommunikativen Legitimität des Parlamentes in Unterunterabschnitt 2.1.4 ist eine der Prämissen, dass Parlamentarierinnen und Parlamentarier zwei Sätze an Präferenzen haben. Zum einen die Präferenzen, welche sie mit ihren Wählerinnen und Wählern teilen. Zum anderen aber auch Präferenzen, welche nur sie selber hegen und ihre Wählerinnen und Wähler nicht. Diese suboptimale Kongruenz der Präferenzen zwischen der Wählerschaft und den Parlamentarierinnen und Parlamentariern bildet den Kern des Spannungsverhältnisses zwischen Prinzipal und Agent. Auch wenn diese Prämisse empirisch immer wahr ist ${ }^{14}$, ist

14 Wenn dem Agenten, in diesem Falle Parlamentarierinnen und Parlamentariern, Akteursstatus und damit die Eigenschaft des sinngeleiteten Handelns unterstellt wird, können dessen Präferenzen nie perfekt mit den 
damit noch nichts über die Natur der Präferenzen der Parlamentarierinnen und Parlamentarier ausgesagt. Eben diese Natur der Präferenzen ist aber wichtig, wenn es darum geht, das Handeln von Parlamentarierinnen und Parlamentariern im Kontext der öffentlichen Kommunikation zu verstehen.

Bisher habe ich argumentiert, dass Parlamentarierinnen und Parlamentarier handlungsfähige Akteure sind - eine nicht übertrieben gewagte Prämisse. Die Natur der Medien aber habe ich bisher nicht explizit thematisiert, sondern Medien eher ad hoc eingeführt und behandelt. Obschon im Zuge der Medienkrise der vollends automatisierte und autonome, durch Computer hergestellte Journalismus Einzug in Redaktionen hält (Carlson 2015; Clerwall 2014), erachte ich den Grossteil journalistischer Arbeit als nach wie vor sinngeleitetes Handeln ${ }^{15}$, und zwar mindesten in doppeltem Sinn. Auf der Meso-Ebene sind Medien Organisationen, welche strategisch handeln und journalistischen Output grundsätzlich steuern. Auf der Mikro-Ebene bestehen Medien (weitgehend) aus individuellen Journalistinnen und Journalisten, die innerhalb der Medienorganisation handeln, d.h. öffentliche Kommunikation herstellen. Auf der Mikro-Ebene sind Journalistinnen und Journalisten entsprechend auch Akteure mit eigenen Präferenzen, die nicht bloss passiv widergeben, sondern aktiv handeln. Auch für Medien stellt sich entsprechend die Frage, wie die Präferenzordnungen ausfallen, wenn es um Berichterstattung rund um das Parlament geht.

In dem Beziehungs-Dreieck von Wählerschaft, Parlament und Medien handelt es sich also um drei Sätze an Präferenzen, und diese Sätze an Präferenzen können sich mehr oder minder überschneiden. Um das Verhältnis dieser Präferenz-Sätze zueinander plastischer zu machen, können die Präferenzen als Mengen und die Überschneidungen der Präferenzen als Teilmengen gedacht werden, wie dies in Abbildung 3 visualisiert ist.

Die in Abbildung 3 dargestellten Schnittmengen sind nur schematischer Natur und geben nicht die empirische Realität wider. Dennoch sind damit die Beziehungen dieser drei PräferenzSätze erschöpfend und in weitreichender Hinsicht zusammengefasst. Jedes der drei Teilsysteme

Präferenzen des Prinzipals übereinstimmen. Die Genese von Präferenzen ist nicht ein exogener Vorgang, der für alle Akteure auf die gleiche Art verläuft und dieselben Ergebnisse produziert. Präferenzen entstehen zumindest in Teilen endogen, sind also Folge des Handelns der Akteure im Rahmen ihrer jeweiligen Lebenswelten. Daraus können wohl ähnliche, aber niemals identische Präferenzen in Betreff des Sozialen im Allgemeinen, des Politischen im Besonderen entstehen. Ein weiterer Grund für nicht-perfekte Übereinstimmung von Präferenzen sind Informationsasymmetrien, die immer vorhanden sind. Die Idee der perfekten Informiertheit des Prinzipals ist empirisch nicht umsetzbar; es ist lediglich eine asymptotische Fiktion.

15 Bei automatisierten, durch Computer hergestellten Formen journalistischer Arbeit handelt es sich um eine Anwendung künstlicher Intelligenz. Allerdings bedeutet künstliche Intelligenz noch keinen Akteursstatus, denn damit ist lediglich gemeint, dass Software die fix definierten Ziele, also Präferenzen, so gut wie möglich erreicht und dabei zielorientiert mit der (unvorhergesehenen) Umgebung interagiert. Diese Form von Intelligenz ist aber kein vernunftgeleitetes, autonomes Handeln (Brooks 1991). 
Abbildung 3: Schematische Darstellung der Präferenz-Schnittmengen der Wählerschaft, der Parlamentarierinnen und Parlamentarier sowie der Medien.

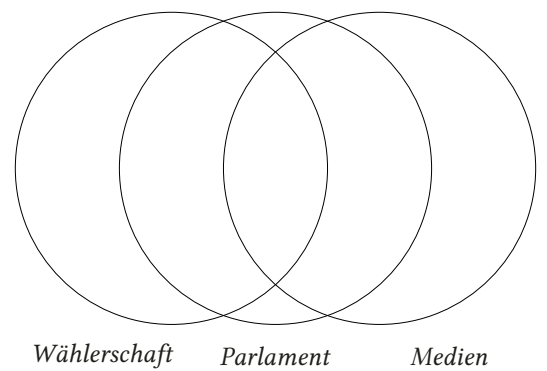

Quelle: Eigene Darstellung.

- die Wählerschaft, das Parlament (also die Parlamentarierinnen und Parlamentarier) und die Medien verfolgen bestimmte Präferenzen welche nicht den Präferenzen der je anderen zwei Teilsysteme entsprechen. Weiter verfolgen die Wählerschaft und das Parlament Präferenzen, welche nicht den Präferenzen der Medien entsprechen. Das Parlament und die Medien verfolgen Präferenzen, welche handkehrum nicht den Präferenzen der Wählerschaft entsprechen. Schliesslich gibt es noch bestimmte Präferenzen, welche die Wählerschaft, das Parlament und die Medien gemeinsam verfolgen. Im Kontext des Verhältnisses von Wählerschaft zum Parlament als Prinzipal-Agent-Beziehung sind alle Teilmengen des Parlamentes, welche nicht mit der Wählerschaft geteilt sind, problematisch, denn dabei handelt es sich um Präferenzen, welche zu der Erreichung der Präferenzen des Prinzipals, d.h. der Wählerschaft, bestenfalls nicht beitragen, ihnen schlimmstenfalls einen Abbruch tun.

Die schematische Unterscheidung nach Präferenzen der drei Teilsysteme sagt noch nichts über die konkreten spezifischen Präferenzen von Wählerschaft, Parlament und Medien aus. Die spezifischen Ziele, welche die Akteure der jeweiligen Teilsysteme verfolgen, können denn auch grundsätzlich unendlich vielfältig sein. Es ist darum nicht möglich, a priori alle Präferenzen spezifisch aufzufächern und zu vergleichen. Vielmehr ist die Frage, was für Ziele die jeweiligen Akteure verfolgen, eine empirische. Doch auch empirisch macht es wenig Sinn, nach allen möglichen handlungsleitenden Präferenzen zu fragen, denn das würde schnell in einer nicht bewältigbare Komplexität münden: Die kompletten Motivstrukturen aller individuellen Akteure müssten in ihrer Gänze berücksichtigt werden, was, offensichtlicherweise, nicht möglich ist. Es ist also weder möglich noch sinnvoll, die Präferenzen der Wählerschaft, der Parlamenta- 
rierinnen und Parlamentarier sowie der Medien komplett theoretisch zu modellieren oder empirisch zu erfassen. Es ist aber möglich, die schematische Darstellung der Schnittmengen aus Abbildung 3 zu einer Heuristik weiterzuentwickeln, welche es erlaubt, die unterschiedlichen Präferenzen als unterschiedliche Familien von Präferenzen im Kontext der öffentlichen Kommunikation über das Parlament zu verstehen. Das Verhältnis konkreter Präferenzen zu generellen Präferenzfamilien ist, in logischer Hinsicht, ein einfaches. Als Präferenzfamilie erachte ich eine Kategorie von Präferenzen, welche alle möglichen konkreten Präferenzen in allen möglichen konkreten Entscheidungssituationen umfasst, welche ein gemeinsames höherrangiges Ziel verfolgen. Präferenzfamilien sind also eine Meta-Präferenz, auf welche die einzelnen konkreten Präferenzen in unterschiedlichen Entscheidungssituationen ausgerichtet sind. Das Verhältnis von Präferenzfamilien zu konkreten Präferenzen lässt sich anhand eines banalen Beispieles veranschaulichen. Angenommen, eine Person XY beschliesst, im Sommer ins Ausland in der Urlaub zu reisen - somit hat die Person XY Sommerferien als übergeordnetes Meta-Ziel (im Gegensatz zum Meta-Ziel, zuhause zu bleiben). Um auf das übergeordnete Meta-Ziel der Sommerferien hinzuarbeiten, muss die Person XY noch eine reihe konkreter Präferenzen in konkreten Entscheidungssituationen umsetzen, beispielsweise den genauen Ferienort bestimmen. Bei der Bestimmung des Ferienortes kann die Person XY unter vielen Möglichkeiten wählen - darunter auch, als Ferienort das Zuhause auszuwählen und damit nicht fortzureisen. Aber wenn die Annahme ist, dass Person XY im Sinne der Präferenzfamilie der Sommerferien handelt und die Meta-Präferenz der Sommerferien darin besteht, ins Ausland zu reisen, dann wird Person XY bei der konkreten Bestimmung des Ferienortes die Option des Zuhause-Bleibens entweder gar nicht unter den konkreten Präferenzen führen, oder wenn doch, dann sehr tief in der Rangordnung.

Die Präferenzfamilien im Rahmen parlamentarischen Handelns sind in Abbildung 4 visualisiert.

Die erste Präferenzfamilie ist durch die Schnittmenge der Präferenzen der Wählerschaft mit den Präferenzen des Parlamentes gegeben (in Abbildung 4 hellgrau und dunkelgrau eingefärbt). Diese Schnittmenge macht das aus, was aus der Perspektive der Prinzipal-Agent-Beziehung normativ wünschenswert ist - diese Schnittmenge ist jenes parlamentarische Handeln, welche dem Auftrag des Prinzipals entspricht. Diese Präferenzfamilie nenne ich die Politiklogiken. $\mathrm{Zu}$ dieser ersten Präferenzfamilie der Politiklogiken gehört auch ein Teil der Präferenzen der Medien (die dunkelgraue Schnittmenge in Abbildung 4): Das ist jener Teil der drei Sätze an Präferenzen von Wählerschaft, Parlament und Medien, welcher von allen drei Sätzen geteilt wird. Diese Schnittmenge der drei Sätze bedeutet, dass im Rahmen des parlamentarischen 
Abbildung 4: Schematische Darstellung der Politiklogiken und der Medienlogiken im Rahmen parlamentarischen Handelns.

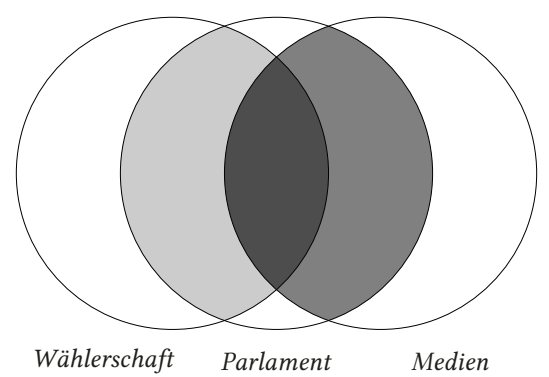

Legende: Hellgrau und dunkelgrau = Politiklogiken haben Priorität im parlamentarischen Handeln; mittleres grau $=$ Medienlogiken haben Priorität im parlamentarischen Handeln. Quelle: Eigene Darstellung.

Handelns nach wie vor die Politiklogiken zum Ausdruck kommen. Allerdings fällt nicht die gesamte Präferenz-Schnittmenge zwischen Parlament und Medien in diese Kategorie: Es gibt eine Schnittmenge zwischen Parlament und Medien, an welcher die Präferenzen der Wählerschaft nicht beteiligt sind (rot eingefärbt in Abbildung 4). Diese Schnittmenge parlamentarischen Handelns, bei der die Präferenzen der Wählerschaft nicht zum Ausdruck kommen, nenne ich die Präferenzfamilie der Medienlogiken.

Die Konzepte von Medienlogik und Politiklogik sind nicht grundsätzlich neu. Insbesondere das Konzept der Medienlogiken ist spätestens seit Altheide und Snow (1979) prominent in der Kommunikationswissenschaft vertreten. Altheide und Snow (ebd., S. 10) beschreiben Medienlogik folgendermassen:

In general terms, media logic consists of a form of communication; the process through which media present and transmit information. Elements of this form include the various media and the formats used by these media. Format consists, in part, of how material is organized, the style in which it is presented, the focus of emphasis on particular characteristics of behavior, and the grammar of media communication. Format becomes a framework or a perspective that is used to present as well as interpret phenomena. [Kursiv im Original]

In dieser Definition wird Medienlogik derart beschrieben, dass Medien nicht als bloss passiver und neutraler Spiegel der Gesellschaft zu verstehen sind, sondern, dass Medien als handelnde 
Akteure Inhalte selektieren, interpretieren und darstellen. Dieses Postulat ist der Kern des Konzeptes der Medienlogiken, und es ist im Grunde wichtiger als die Frage, wie genau die konkreten Medienlogiken ausfallen. Wenn Medien nämlich als handelnde Akteure konzipiert werden und ihnen dabei auf der Mikro-Ebene kontingentes Handeln unterstellt wird, dann bedeutet das, dass es keine universalen und raum-zeitlich verfestigten Medienlogiken gibt. Zwar wurde schon in der ersten Hälfte des 20. Jahrhunderts bemerkt, dass bestimmte Faktoren wie Neuigkeit, Konflikt und geografische Nähe relevant in der journalistischen Arbeit sind (Lippmann 1922; Warren 1934). Diese generellen Nachrichtenfaktoren (oder Nachrichtenwerte) beschreiben aber lediglich grobe Gemeinsamkeiten in der thematischen Berichterstattungspraxis, nicht aber universale Präferenzordnungen der Medien. Wenn solche groben Nachrichtenwerte (egal, wie umfangreich deren Katalog ist) als universal missverstanden werden, werden im Mindesten zwei Fehlschlüsse begangen. Erstens geht damit der Akteursstatus der Medien verloren. Medien werden zu passiven Herstellern von Berichterstattung, und die Regeln für das Herstellen der Berichterstattung sind komplett exogen, also ereignisgetrieben, wie dies sehr explizit u.a. bei Galtung und Ruge (1965) als Prämisse postuliert ist. Einige Autoren versuchen allerdings, den Handlungscharakter von Journalistinnen und Journalisten durch Erweiterungen dieser Nachrichtenwerttheorie wieder ein Stück weit einzubringen, etwa Staab (1990, S. 96), indem er in einem «Finalmodell» Journalistinnen und Journalisten als Quelle der Nachrichtenwerte sieht, oder auch Kepplinger und Ehmig (2006), indem sie beobachten, dass bei unterschiedlichen Zeitungstypen unterschiedliche Nachrichtenfaktoren bevorzugt werden. Der Ansatz der Nachrichtenwerte ist, zweitens, ahistorisch, denn, wie ich in Unterunterabschnitt 2.2.1 argumentiert, wandeln sich Medien konstant und manchmal, wie in der aktuellen Phase, krisenartig. Wenn sich Medien im Besonderen und die Gesellschaft im Allgemeinen wandeln und Medien handlungsfähige Akteure sind, dann ist auch anzunehmen, dass die Präferenzen der Medien nicht universal und konstant sind, sondern einem Wandel unterliegen. Diese Historizität der Medienlogiken als Präferenzen handelnder Akteure wird genauer in Unterabschnitt 3.2 diskutiert.

Das Konzept der Politiklogiken als Gegenstück zu Medienlogiken findet bisweilen Verwendung, aber oftmals werden Politiklogiken dabei schlicht in einer ex negativo-Definition als Nicht-Medienlogiken verstanden (Landerer 2013). Versuche, Politiklogiken im Kontext politischer Kommunikation doch explizit zu beschreiben, beispielsweise bei Mazzoleni (1987), T. Meyer (2002), Strömbäck (2008) und Strömbäck und Esser (2009), münden oft in einer generischen Beschreibung von Politik und schreiben der Medienlogik pauschal einen negativen oder zumindest nicht förderlichen Effekt auf politische Entscheidungsfindung zu. Ein solches 
Verständnis von Politiklogiken ist aber problematisch, weil Politik damit auf «Politics», also auf den Politikprozess, reduziert wird und a priori ein Antagonismus zwischen Medienlogiken und Politiklogiken vermutet wird: Medienlogiken beeinträchtigen den Politikprozess. In einer solchen Lesart bedeuten Medienlogiken automatisch eine Bedrohung für Politiklogiken, und dieser vermeintliche Antagonismus als Grundlage für empirische Forschungsprogramme bestätigt in zirkulärer Manier automatisch, dass Medienlogiken empirisch in der Tat den Politiklogiken zum Nachteil gereichen, sobald das wie auch immer empirisch bestimme Mass für Medienlogiken höher als Null ist.

Das Konzept der Politiklogiken, wie ich es hier vorschlage, umgeht diesen Fehlschluss. Ich beschreibe nicht universale Prozesscharakteristika des Politischen, sondern definiere Politiklogiken enger im Kontext der öffentlichen Kommunikation über das Parlament als die Schnittmenge der Präferenzen von Wählerschaft und Parlament. Ein Teil dieser Schnittmenge der Präferenzen von Wählerschaft und Parlament kann, wie in Abbildung 4 visualisiert, einen Teil der Präferenzen der Medien enthalten. Das bedeutet, dass Politiklogiken - also das Handeln der Parlamentarierinnen und Parlamentarier im Sinne der Präferenzen ihrer Wählerschaft in der Logik der Beziehung von Prinzipal und Agent - auch dann erhalten bleiben können, wenn gleichzeitig auch noch ein Teil der Präferenzen der Medien erfüllt ist.

Was aber bedeuten Politiklogiken im konkreten parlamentarischen Alltagsgeschehen? Sind Politiklogiken dann erfüllt, wenn Parlamentarierinnen und Parlamentarier perfekt wissen, was ihre jeweilige Wählerschaft möchte, und sie diese Präferenzen der Wählerschaft perfekt umsetzen? Nein. Eine solche Forderung würde, wie ich später in Unterabschnitt 3.3 beschreibe, ein Handlungsomdell bedeuten, welches realistischerweise unsinnig ist; es ist nicht möglich, dass Parlamentarierinnen und Parlamentarier permanent wissen können, was ihre Wählerschaft genau möchte. Im Sinne der Politiklogiken handeln meint darum stattdessen, dass Parlamentarierinnen und Parlamentarier im Glauben und nach bestem Wissen und Gewissen handeln, dass sie so handeln, wie es ihre Wählerschaft, wie es also der Prinzipal, möchte. Diese Anforderung bedeutet, dass Parlamentarierinnen und Parlamentarier einerseits aktiv reflektieren können und sollen, wie sie zugunsten ihrer Wählerschaft handeln können. Andererseits, und dominanterweise, meint Handeln nach Politiklogiken allerdings, dass Parlamentarierinnen und Parlamentarier aus aufrichtiger politischer Überzeugung handeln. Wenn Parlamentarierinnen und Parlamentarier im Parlament also handeln wie sie handeln, weil sie der Meinung sind, dass dieses Handeln normativ politisch richtig ist, dann handeln sie damit im Sinne der Politiklogiken. Es ist natürlich möglich, dass der Prinzipal, also die jeweilige Wählerschaft, im Nachhinein nicht ganz einverstanden mit diesem konkreten Handeln ist. Parlamentarisches Handeln aus 
normativer politischer Überzeugung ist aber die eigentliche normative Präsupposition für das Prinzipal-Agent-Verhältnis zwischen Volk und Parlament.

\subsubsection{Forschungsfragen}

Die bisherigen einleitenden Ausführungen sollten mit dem Argument, dass erst öffentliche Kommunikation prozeduralisierte Volkssouveränität ermöglicht, verständlich machen, warum das Parlament in einem besonderen Verhältnis zu massenmedial hergestellter öffentlicher Kommunikation steht. Die Herleitung dieser kommunikativen Legitimität des Parlamentes ist aber auch mit den Verweisen auf die Medienkrise und auf die Natur der Präferenzfamilien von Politiklogiken und Medienlogiken noch nicht spezifisch genug, um ein konkretes empirisches Forschungsprojekt durchzuführen. Darum werden in diesem Abschnitt die konkreten Forschungsfragen formuliert, deren Beantwortung das Ziel der vorliegenden Arbeit ist.

Die handlungstheoretische Prämisse dieser Arbeit ist, wie aus der vorangehenden Argumentation hervorgeht, die Annahme, dass die involvierten Akteure Präferenzen haben, und, dass die Akteure im Sinne dieser Präferenzen handeln. In Unterunterabschnitt 2.2.2 unterscheide ich zwei Präferenzfamilien im Rahmen parlamentarischen Handelns, die Politiklogiken und die Medienlogiken, wobei die Politiklogiken die Schnittmenge der Präferenzen der Wählerschaft und der Parlamentarierinnen und Parlamentarier abbilden, und die Medienlogiken die Schnittmenge der Präferenzen der Parlamentarierinnen und Parlamentarier und der Medien, welche ohne Bezug zu den Präferenzen der Wählerschaft sind. Dieses handlungstheoretische Verständnis bildet das konzeptuelle Gerüst der vorliegenden Arbeit. Dieses Gerüst beschreibt aber nur, dass und warum es unterschiedliche Präferenzen und Präferenzfamilien im Kontext parlamentarischen Handelns grundsätzlich gibt - in welchem Masse diese Präferenzfamilien konkreterweise ausgebildet sind, ist empirisch zu klären. Dies soll anhand der ersten zwei Forschungsfragen geschehen:

- FF1: In welchem Masse handeln Parlamentarierinnen und Parlamentarier nach Politiklogiken und in welchem Masse nach Medienlogiken?

- FF2: In welchem Masse handeln fournalistinnen und fournalisten in der Berichterstattung über das Parlament nach Medienlogiken und in welchem Masse nach Politiklogiken?

Diese zwei Forschungsfragen mögen auf den ersten Blick etwas unscheinbar wirken, sie engen aber das Forschungsvorhaben schon in bedeutendem Masse ein. Eine notwendige Bedingung für repräsentative Demokratie als prozeduralisierte Volkssouveränität ist, wie in Unterunterabschnitt 2.1.4 zur kommunikativen Legitimität des Parlamentes argumentiert, die grundsätzliche 
öffentliche Sichtbarkeit des Parlamentes. Die Minimalanforderung, dass das Parlament über massenmedial hergestellte Kommunikation öffentlich sichtbar sein soll, kann in der Schweiz als recht eigentlich erfüllt angesehen werden (Ettinger 2013). Entsprechend wäre eine Forschungsfrage, welche danach fragt, ob die öffentliche Sichtbarkeit des Parlamentes höher als Null ist, rasch beantwortet. Die ersten beiden Forschungsfragen zielen darum nicht auf diese grundsätzliche öffentliche Sichtbarkeit des Parlamentes ab. Stattdessen fokussieren die Forschungsfragen 1 und 2 auf die Beschaffenheit des Handelns der Parlamentarierinnen und Parlamentarier sowie der Journalistinnen und Journalisten. Wenn auch die Forschungsfragen dabei eine Dichotomie suggerieren - entweder Politiklogiken oder Medienlogiken - , eröffnen die Fragestellungen ein höheres Mass an Komplexität, wie in Abbildung 4 dargestellt ist. Die Präferenzen der Medien und die Präferenzen des Parlamentes haben eine Schnittmenge, welche unter die Politiklogiken fällt. Das bedeutet, dass Parlamentarierinnen und Parlamentarier auf eine solche Art handeln können, dass die Präferenzen der Medien erfüllt sind, aber gleichzeitig auch die Präferenzen der Wählerschaft. Wenn die Präferenzen der Medien erfüllt sind, hat das also nicht automatisch zur Folge, dass Medienlogiken Vorrang vor Politiklogiken haben.

Die ersten zwei Forschungsfragen spezifizieren keine zeitliche Komponente. Sie implizieren stattdessen, dass das Handeln von Medien und Parlament konstant ist. Nun ist Teil des Entdeckungszusammenhanges dieser Arbeit, wie in Unterunterabschnitt 2.2.1 argumentiert, der Umstand, dass die Schweiz sich gegenwärtig in einer Medienkrise befindet. Darum drängt sich eine weitere Forschungsfrage auf:

- FF3: Wandeln sich die Präferenzen von Parlamentarierinnen und Parlamentariern sowie von fournalistinnen und fournalisten im Zuge der Medienkrise?

Diese drei Forschungsfragen bilden das Fundament für das Forschungsprogramm der vorliegenden Arbeit. Ausgehend von diesen Forschungsfragen wird in Abschnitt 3 die genauere theoretische Rahmung besprochen und es werden die empirisch zu prüfenden Hypothesen formuliert. Zunächst aber skizziere ich in Unterabschnitt 2.3 den generellen Forschungsstand zu den drei Forschungsfragen. 


\subsection{Forschungsstand}

Die Diskussion des Forschungsstandes zu einem Forschungsbereich kann, grob zusammengefasst, eine von zwei Funktionen haben. Die erste dieser Funktionen ist, bestehende Forschung zu einem spezifischen Sachverhalt umfassend und systematisch zu analysieren, um die vorhandenen Einzelbefunde auf einer höherrangigen Ebene aussagekräftig zusammenzufassen. Typische Beispiele für diese Art von Analyse des Forschungsstandes sind systematische Reviews und, falls bestimmte Messgrössen oder Effekte über mehrere Studien hinweg statistisch verglichen werden, Metaanalysen (Akobeng 2005; Uman 2011).

Die zweite Funktion der Besprechung des Forschungsstandes ist weniger eine systematische und möglichst umfassende Analyse der bestehenden Forschung zu einer spezifischen Fragestellung, sondern mehr eine gut begründete Erklärung, warum ein bestimmtes Forschungsvorhaben notwendig ist. Der in diesem Abschnitt zu diskutierende Forschungsstand hat die zweite dieser Funktionen: Das Ziel ist, die wesentlichen Züge der bestehenden relevanten Forschung zusammenzufassen, um schliesslich die Notwendigkeit der vorliegenden empirischen Arbeit als Schliessung einer bestimmten Lücke herzuleiten. Eine derartige Diskussion des Forschungsstandes als narratives Review birgt Gefahren (Cipriani und Geddes 2003; Collins und Fauser 2005), vor allem den Umstand, dass das Vorgehen beim narrativen Review stark subjektiv geprägt sein kann, sodass das Ganze schlimmstenfalls zu einer Übung im Confirmation Bias (Nickerson 1998) verkommen kann. Um dieser Gefahr ein Stück weit vorzubeugen, ist das kompakte narrative Review in diesem Abschnitt nicht komplett unsystematisch, sondern erfolgt anhand der folgenden Punkte:

- Das Ziel ist, den Forschungsstand zu jeder der drei Forschungsfragen zusammenzufassen (Unterabschnitte 2.3.1, 2.3.2 und 2.3.3).

- Für jede Forschungsfrage sollen zwei Aspekte berücksichtigt werden:

- Forschung zur Forschungsfrage im engeren Sinn.

- Forschung zur Forschungsfrage im breiteren Sinn.

- Abschliessend wird in Unterunterabschnitt 2.3.4 zusammengefasst, warum und wie die vorliegende Arbeit gegeben der bestehenden Forschung eine relevante Ergänzung darstellt.

Forschung zu den Forschungsfragen im «engeren Sinn» meint jene Forschung, welche die Forschungsfragen recht explizit und direkt betrifft, also die Präferenzlagen von Parlament (Forschungsfrage 1) und Medien (Forschungsfrage 2) sowie den Wandel der Präferenzlagen im 
Zuge der Medienkrise (Forschungsfrage 3) adressiert. Der «breitere Sinn» dieser Forschungsfragen betrifft Forschung, welche zwar nicht auf der expliziten Idee der Präferenzlagen und Medienkrise fusst, aber dennoch empirisch Fragen behandelt, welche impliziterweise in den Bereich dieser Forschungsfrage fallen. Hierunter fällt Forschung, welche parlamentarisches Handeln beschreibt, dabei aber nicht explizit Handeln im Sinne von Präferenzen als handlungstheoretischen und normativen Ausgangspunkt formuliert. Forschung mit Bezug zur Schweiz, im engeren wie auch im breiteren Sinn, wird für jede Forschungsfrage explizit mitberücksichtigt.

Die Literatursuche habe ich gemäss den folgenden Kriterien durchgeführt:

- Für die Literatursuche habe ich ausschliesslich den Indexierungs-Dienst «Google Scholar» verwendet, anstatt einzelne Zeitschriften, Repositories o.ä. separat manuell zu durchsuchen. Bei Google Scholar sind schätzungsweise zwischen $85 \%$ und $100 \%$ aller englischsprachigen wissenschaftlichen Publikationen (Aufsätze in Journals, Bücher, Working Papers, und so fort) indexiert (Gehanno, Rollin und Darmoni 2013; Khabsa und Giles 2014).

- Die Suche in Google Scholar habe ich ausschliesslich auf Englisch und auf Deutsch durchgeführt.

- Für Literatur zu den ersten beiden Forschungsfragen im engeren Sinn habe ich folgende Stichworte verwendet:

- Englisch: «preferences», «parliament», «legislative», «media», «mp», «journalist», «congress», «senate», «house of representatives».

- Deutsch: «präferenzen», «parlament», «legislative», «medien», «parlamentarier», «journalist», «nationalrat», «ständerat», «congress», «senate», «house of representatives», «bundestag».

- Für Literatur zu den ersten beiden Forschungsfragen im breiteren Sinn habe ich folgende Stichworte verwendet:

- Englisch: «parliament», «legislative», «media», «mp», «journalist», «congress», «senate», «house of representatives», «influence», «interaction», «agenda», «effect», «topics».

- Deutsch: «parlament», «legislative», «medien», «parlamentarier», «journalist», «nationalrat», «ständerat», «congress», «senate», «house of representatives», «bundestag», «einfluss», «interaktion», «wechslwirkung», «agenda», «effekt», «themen».

- Für Literatur zu der dritten Forschungsfrage habe ich die Suchbegriffe für die ersten beiden Forschungsfragen kombiniert zusammengenommen und noch folgende zusätzliche Begriffe gesucht: 
- Englisch: «change», «crisis», «development», «transformation».

- Deutsch: «wandel», «veränderung», «krise», «entwicklung», «transformation».

Die Auswahl der Literatur für den Forschungsstand besteht aber nicht aus der Summe aller Suchtreffer anhand der dargelegten Suchstrategie. Google Scholar zeigt bei fast jeder Art von Suchanafrage Tausende von Ergebnissen an, von denen nur ein kleiner Bruchteil für die Suche wirklich von Belang ist. Somit war im Anschluss an die jeweilige Suche über Google Scholar trotzdem noch eine qualitative Einsicht in die Suchergebnisse von Nöten, um tatsächlich relevante Beiträge herauszufiltern. Ein nicht unwesentlicher Teil der Ergebnisse bezieht sich etwa auf Berichterstattung während Wählkämpfen, was für die vorliegende Arbeit explizit nicht im Fokus des Forschungsinteresses steht.

\subsubsection{Präferenzen der Parlamentarierinnen und Parlamentarier}

Forschung zu Präferenzen von Parlamentarierinnen und Parlamentariern im engeren Sinn hat vor allem für den US-amerikanischen Kontext Tradition. Die zentrale Fragestellung dieser Forschungstradition ist dabei schon früh, ob und in welchem Masse die Präferenzen der Parlamentarierinnen und Parlamentarier mit den Präferenzen ihrer Wählerschaft, der «Constituency», übereinstimmen. W. E. Miller und D. E. Stokes (1963, S. 45) beschreiben das Problem wie folgt:

The fact that our House of Representatives [...] has irregular party voting does not of itself indicate that Congressmen deviate from party in response to local pressure. And even more, the fact that many Congressmen feel pressure from home does not of itself establish that the local constituency is performing any of the acts that a reasonable definition of control would imply.[Kursiv im Original]

Die Befunde der frühen Forschung zu dieser Fragestellung legen nahe, dass die Mitglieder des US-Kongresses ${ }^{16}$ die Präferenzen ihrer Wählerschaft nur bedingt überhaupt einschätzen können und entsprechend grosse Unterschiede zwischen den Präferenzen der Wählerschaft und der Präferenzen des Kongresses vorhanden sind (Meller 1965; W. E. Miller und D. E. Stokes 1963). Diese Schlussfolgerung ist aber nicht auf die 1960er Jahre beschränkt; auch für die nachfolgenden Jahrzehnte zeichnen die Befunde ein anhaltend ähnliches Bild (Bafumi und Herron 2010; Erikson, Luttbeg und Holloway 1975; Levitt 1996; Poole und Rosenthal 1996; Uslaner und Der US-Kongress ist ein Zweikammer-Parlament, bestehend aus dem «House of Representatives» und dem
«Senate», analog zum Schweizer National- und Ständerat. 
R. E. Weber 1979). Diese konstante Diskrepanz zwischen den Präferenzen der Wählerschaft und den Präferenzen der Gewählten deuten Bafumi und Herron (2010) als «Extremismus» der in den Kongress Gewählten: Paradoxerweise setzen sich Kandidierende mit Präferenzen, welche moderater und damit näher an den Präferenzen der Wählerschaft sind, im Wahlkampf nicht gegen Kandiderende mit extremeren Positionen durch. Diese Befunde wurden vereinzelt mit dem Verweis auf unterschiedliche Politikfelder relativiert. Page u. a. (1984) argumentieren beispielsweise, dass die Übereinstimmung der Präferenzen relativ höher sei bei sozialpolitischen Angelegenheiten. Für die Diskrepanz der Präferenzen von Wählerschaft und Gewählten in den USA wird eine Reihe möglicher Erklärungen vorgeschlagen. Bishin (2000) argumentiert, dass Kongressabgeordnete sich nach der Wahl nicht mehr gross für ihre aktuelle Wählerschaft interessieren, sondern sich bereits prospektiv nach den zukünftigen Wählerschaften richten. Zudem gibt es Anzeichen dafür, dass Parlamentarierinnen stärker von den Präferenzen der Wählerschaft abweichen als Parlamentarier, weil sie tendenziell stärker links-liberal in Fragen der Sozialpolitik abstimmen (Poggione 2004; Swers 2002). Allgemein spielen Politikfelder eine Rolle: Die Diskrepanz der Präferenzen ist bei jenen Politikfeldern geringer, welche die Wählerschaft als wichtiger erachtet (Kuklinski und Elling 1977). Die Rolle der Politikfelder geht in der modernen Literatur ein Stück weit verloren, da moderne quantitative Verfahren wie etwa die «Bayesian Item-Response»-Modellierung (Tausanovitch und Warshaw 2013) konzeptuell in der Regel mit eindimensionalen Policy-Räumen arbeiten, bei denen keine inhaltliche Differenzierung nach Politikfeldern stattfindet. Forschung zu parlamentarischen Präferenzen ist nicht auf die USA beschränkt, aber die Forschungstradition ist doch grosso modo eine angelsächsische. Entsprechend sind Studien, welche die parlamentarischen Präferenzen ausserhalb der USA untersuchen, vor allem für Staaten wie Kanada (Kam 2001; Soroka, Penner und Blidook 2009) und das Vereinigte Königreich (Carman 2006; Kam 2001) vorhanden. Diese Studien sind insgesamt aber zaghafter als die Forschung zu der Situation in den USA; die Hauptergebnisse und -argumente dieser Studien sind, dass Präferenzen von Parlamentarierinnen und Parlamentariern und Präferenzen der Wählerschaft überhaupt eine untersuchenswerte Dimension des Parlamentes darstellen. Der Grund dafür ist, dass parlamentarisches Handeln in Staaten jenseits der USA lange Zeit in erster Linie durch die analytische Linse der Parteipolitik verstanden wurde - die Frage nach individuellem parlamentarischen Handeln mit der normativen Perspektive der Präferenzen der Wählerschaft stellte sich lange kaum, da parlamentarisches Handeln als Parteihandeln in Sinne einer «Black Box» verstanden wurde. Einige Studien im Schweizer Kontext plädieren mittlerweile aber für ein Abkommen von diesem parteifokussierten «Black Box»-Verständnis parlamentarischen Handelns. So argumentieren etwa Traber, Hug und Sciarini (2014, S. 209): 
Many sources of variation are quite possibly to be found on the level of individual legislators, which was not taken into account in this analysis, as the units of observation were party votes.

Diese An- und Einsicht in Betreff individueller Präferenzen in der Schweizer Bundesversammlung findet in einigen wenigen weiteren Studien Einzug. Bütikofer und Hug (2010) argumentieren beispielsweise, dass die individuellen Mitglieder des Ständerates nicht bedeutend unterschiedlichere Präferenzen als die Mitglieder des Nationalrates haben, wenn für die Parteizugehörigkeit kontrolliert wird. In einer weiteren Studie attestieren Bütikofer und Hug (2015) den individuellen Mitgliedern des Nationalrates strategisches Handeln bei Abstimmungen. Wenn auch diese Erkenntnis im Grunde trivial und der konzeptuelle Überbau der Studie nicht vollends klar ist ${ }^{17}$, stellt das implizite handlungstheoretische Verständnis eines solchen Vorgehens einen relevanten Fortschritt dar.

Alle Forschung zu Präferenzen von Parlamentarierinnen und Parlamentariern im engeren Sinn hat eine Gemeinsamkeit: Die Präferenzen der Medien spielen in dieser Literatur, so meine Wahrnehmung der Studienlage, so gut wie keine Rolle. Insbesondere die Forschung zum USamerikanischen Kontext arbeitet konzeptuell verhältnismässig explizit mit der Präferenzfamilie der Politiklogiken (also dem Präferenzverhältnis von Wählerschaft und Gewählten), die Präferenzfamilie der Medienlogiken fehlt aber komplett. Die Präferenzfamilie der Medienlogiken im Kontext der Präferenzen von Parlamentarierinnen und Parlamentariern kommt erst in der relevanten Forschung im breiteren Sinn zur Geltung.

$\mathrm{Zu}$ den Präferenzen von Parlamentarierinnen und Parlamentariern im engeren Sinn ist die Forschung für den US-amerikanischen Kontext, wie oben argumentiert, deutlich umfangreicher als die Forschung etwa zu westeuropäischen Staaten. Das Bild sieht für Forschung zu Präferenzen von Parlamentarierinnen und Parlamentariern im breiteren Sinn anders aus, wenn nicht gar umgekehrt: Zwar gibt es einige Literatur für die USA oder, breiter, für angelsächsische Staaten, doch ist die Forschungslinie für den kontinentaleuropäischen Kontext vor allem nach der Jahrtausendwende ausgeprägter. Das bedeutet aber nicht, dass Medienlogiken als Präferenzfamilie in einem breiten Sinn keine Rolle in der auf die USA bezogenen Forschung

17 Die Autorinnen verwenden etwa die Begriffe «strategic» und «sophisticated» synonym, obwohl damit im «Rational Choice»-Verständnis üblicherweise unterschiedliche Arten des Handelns gemeint sind (Shepsle 2010, S. 156-190). Handeln im Allgemeinen und Abstimmen im Besonderen ist dann «sophisticated», zu Deutsch in etwa «ausgeklügelt» oder «hochentwickelt», wenn ein Akteur vorläufig entgegen seinen Präferenzen handelt, um damit zu einem späteren Zeitpunkt seine höchste Präferenz zu realisieren. Im Unterschied dazu ist «strategic», zu Deutsch «strategisches», Handeln ein solches, bei welchem der Akteur versucht, seine Präferenz so gut wie möglich zu realisieren, wenn er antizipiert, dass seine höchste Präferenz unrealistisch ist. Sowohl ausgeklügeltes wie auch strategisches Handeln ist also vorausschauendes Handeln, aber deutlich unterschiedliches vorausschauendes Handeln. 
spielen. So hat etwa T. E. Cook (1990, S. 12) schon vor einiger Zeit die innige Beziehung der Mitglieder des House of Representatives zu den Medien beschrieben:

The media matter to members of the House of Representatives. Media strategies are important activities, not only to get reelected but increasingly to accomplish policyrelated goals in Washington. And it matters that the media have been welcomed into the legislative process.

In diesem Verständnis sind Parlamentarierinnen und Parlamentarier also strategisch handelnde Akteure, welche sich Medien und damit die Präferenzen der Medien als Medienlogiken zu Nutze machen; der Umstand, dass Politikerinnen und Politiker im Allgemeinen und Parlamentarierinnen und Parlamentarier im Besondern einen strategischen Umgang mit den Medien pflegen, wird bisweilen pejorativ als «spin», also Verdrehung, beschrieben (Sellers 2009). Bei der Frage, was für inhaltliche Folgen die Beziehung des US-Kongresses zu den Medien hat, deuten einige Untersuchungen auf schwache bzw. unstete Zusammenhänge. Parlamentarierinnen und Parlamentarier in den USA übernehmen weder systematisch Themen aus den Medien in ihrer parlamentarsichen Arbeit, noch setzen sie systematisch die Themen in den Medien (Baumgartner und Jones 2010, S. 103-125; Edwards III und Wood 1999; Tan und D. H. Weaver 2007). Eher orientieren sich Parlamentarierinnen und Parlamentarier punktuell und ereignisbezogen in strategischer Manier an den Medien. Es ist allerdings unklar, ob diese vagen Befunde der eher spärlich vorhandenen Forschung für den US-Kontext die effektive Situation zuverlässig widergeben. Möglicherweise ist die Forschungslage zu den Präferenzen der Parlamentarierinnen und Parlamentarier im breiten Sinn für den US-Kontext nicht übermässig umfassend, weil der Forschungsfokus im US-Kontext deutlich stärker auf der Exekutive als auf der Legislative liegt. Davon zeugen Konzepte wie etwa der «CNN-Effekt» (Robinson 2005) oder die «Indexing-Hypothese» (Bennett 1990), welche zu gebräuchlichen alltagstauglichen Begriffen jenseits des sozialwissenschaftlichen Diskurses avanciert sind. Auch ist die Forschung zum US-amerikanischen Kontext von einer möglichen Schwäche hinsichtlich der Forschungsanlage betroffen: Das Handeln des Parlamentes wird in den meisten Untersuchungen als pars pro toto anhand der parlamentarischen Anhörungen, der sogenannten «congressional hearings», analysiert. Das Problem mit Anhörungen ist, dass sie oftmals lediglich symbolpolitischen Charakter haben (Edelman 1985, S. 136) und entsprechend das an sich facettenreiche parlamentarische Handeln nur sehr unvollständig abbilden. Dass die Art und Weise, wie das parlamentarische Handeln im Zusammenhang mit Medien und Medienlogiken gemessen wird, eine wichtige Rolle spielt, demonstrieren Van Aelst und Walgrave (2011). Die Autoren zeigen auf, dass Parlamentarierinnen und Parlamentarier in Belgien, den Niederlanden, Schweden und Dänemark 
subjektiv einschätzen, dass die Inhalte der Medien stark die Inhalte der jeweiligen Parlamente prägen. Dieser individuelle Einschätzung der Parlamentarierinnen und Parlamentariern, so die Autoren weiter, decke sich nicht mit den inhaltsanalytischen Befunden, welche zwar auch Zusammenhänge finden, aber eher schwache und punktuelle. Solche schwachen Zusammenhänge beobachten etwa Noije, Kleinnijenhuis und Oegema (2008) für die Niederlande und für das Vereinigte Königreich; die Befunde von Walgrave, Soroka und Nuytemans (2008) für Belgien sind sehr ähnlich. Einen der Gründe, warum die Befunde eher schwache und punktuelle Zusammenhänge nahelegen, liefern Van Aelst, Sehata und A. V. Dalen (2010): In einer Befragung von Parlamentarierinnen und Parlamentariern in Belgien, den Niederlanden, Schweden, Norwegen und Dänemark zeigt sich, dass Parlamentarierinnen und Parlamentarier nicht universal gleich viel Kontakt mit Journalistinnen und Journalisten haben. Die Forschung zur ersten Forschungsfrage im breiteren Sinn ist für den europäischen Kontext insgesamt relativ facettenreicher als für die USA, aber auf der inhaltsanalytischen Ebene stützt sich auch die Forschung für den europäischen Kontext meistens auf eine pars pro toto-Heuristik, um das parlamentarische Handeln zu analysieren, was erneut die Frage der Generalisierbarkeit der Ergebnisse aufdrängt. In dieser Hinsicht findet aber Fortschritt statt, denn eine Reihe neuerer Untersuchungen interessiert sich explizit nur für einen Teil des parlamentarischen Handeln: Die sogenannten parlamentarischen Fragestunden. Fragestunden sind Gelegenheiten für Parlamentarierinnen und Parlamentarier, Fragen an die Regierung zu richten, welche diese entweder direkt vor Ort im Parlament mündlich oder schriftlich zu beantworten hat. Fragestunden existieren in zahlreichen europäischen Staaten, so auch in der Schweiz (Russo und Wiberg 2010). Studien, welche explizit den Zusammenhang von Fragestunden und Medien behandeln, sind nicht darum ein Fortschritt, weil Fragestunden bisher nie untersucht worden wären; einige pars pro toto-Studien, z.B. Walgrave, Soroka und Nuytemans (2008), analysieren durchaus auch Fragestunden. Die neueren, nur auf Fragestunden fokussierten Studien sind ein Fortschritt gerade weil sie ganz explizit nur Fragestunden untersuchen und es unterlassen, diese eine parlamentarische Arena auf das gesamte Parlament zu verallgemeinern. Somit sind Studien, welche diese Generalisierung nicht vornehmen, handlungstheoretisch plausibler, denn es ist nicht selbstverständlich, dass das parlamentarische Handeln in einem Teilbereich des Parlamentes ohne Weiteres dem parlamentarischen Handeln in anderen Teilbereichen entspricht - dieser fundamentalen handlungstheoretische Komponente des Parlamentes, der Rolle der institutionellen Konfigurationen der unterschiedlichen parlamentarischen Arenen, widme ich mich ausführlicher im Theorieteil dieser Arbeit in Unterabschnitt 3.3. Studien, welche sich explizit parlamentarischen Fragestunden widmen, z.B. Walgrave und Vliegenhart (2011) für Belgien 
und Dänemark, Van Aelst und Vliegenthart (2014) und Van Santen, Helfer und Van Aelst (2013) für die Niederlande, Frankreich und Deutschland sowie Vliegenthart und Montes (2014) für die Niederlande und Spanien, kommen zum Schluss, dass Parlamentarierinnen und Parlamentarier in ihren Fragen teilweise Bezug auf aktuelle Inhalte der Medien nehmen und, dass jene Fragen, welche Bezug auf aktuelle Inhalte der Medien nehmen, wahrscheinlicher im Nachhinein von den Medien als Berichterstattungsthema aufgegriffen werden. Forschung, welche die Präferenzen von Parlamentarierinnen und Parlamentariern im Kontext parlamentarischen Handeln in einem breiteren Sinn für die Schweiz untersucht, ist eher selten, aber nicht ganz inexistent. So zeigt Landerer (2014) auf, dass nicht alle Mitglieder des Nationalrates in gleichem Ausmass darauf bedacht sind, mit Medien strategisch umzugehen, um politische Ziele zu erreichen: Mitglieder der Polparteien SP, SVP und der GPS nutzten Medien stärker, um ihre Ziele zu erreichen. Diese Untersuchung ist in Betreff der Forschungsanlage aber heikel, da die Interviews, welche der Autor geführt hat, über einen längeren Zeitraum stattfanden und explizit unterschiedliche politische Geschäfte betreffen. Somit ist nicht klar, ob die Ergebnisse wirklich eine Aussagekraft haben, da sich die interviewten Parlamentarierinnen und Parlamentarier zu unterschiedlichen Dingen geäussert haben. Zudem ist die Einteilung bestimmter Parteien in die Kategorie «Polparteien» heikel, da nicht begründet; somit entsteht der Eindruck einer gewissen forschungslogischen Zirkularität. Cammarano (2013, S. 183) kommt bei der Frage, in welchem Masse Parlamentarierinnen und Parlamentarier Kontakte zu Medienschaffenden pflegen, zu einem ähnlichen Ergebnis wie Van Aelst, Sehata und A. V. Dalen (2010):

Die Analysen haben gezeigt, dass jeweils lediglich ein kleiner Teil der befragten Parlamentsmitglieder häufige Kontakte zu Medienschaffenden aufweist. Dieser Befund deutet möglicherweise darauf hin, dass lediglich ein kleiner Teil der Parlamentsmitglieder die Berichterstattung direkt oder indirekt beeinflusst.

Dieser Befund mag wenig überraschend sein, weil er für unser Alltagsverständnis sehr plausibel ist. Dennoch hat dieser Befund einen nicht zu unterschätzenden Wert, denn er verdeutlicht nochmals, dass eine Konzipierung des parlamentarischen Handelns nicht nur auf der abstrahierten Ebene von Parteien gründen soll, sondern, dass parlamentarisches Handeln in erster Linie als Handeln individueller Akteure gedacht werden muss. Wenn auch die direkten Kontakte von Parlamentarierinnen und Parlamentarierin zu Journalistinnen und Journalisten nicht sehr häufig sind, sind sich Parlamenterierinnen und Parlamentarier dennoch bewusst, dass Medienpräsenz für ihre politische Karriere wichtig ist, falls die frühe Untersuchung von Saxer und Hofer (1992, S. 95) noch Gültigkeit besitzt: Über 90\% der 168 befragten Parlamentarierinnen 
und Parlamentarier schätzen ein, dass Präsenz in den Medien eher bis sehr wichtig ist. Der Umstand, dass Medienpräsenz als so wichtig erachtet wird, erklärt vielleicht auch, warum die Parlamentarierinnen und Parlamentarier aus derselben Studie (ebd., S. 122) einen Einfluss von Journalistinnen und Journalisten spüren; eher nicht einen direkten Einfluss durch persönliche Kontakte, sondern stärker durch die Wahrnehmung der Berichterstattung sowie durch die vermutete öffentliche Meinung infolge der Berichterstattung.

\subsubsection{Präferenzen der Medien}

Forschung, welche Präferenzen von Parlamentarierinnen und Parlamentariern im engeren Sinne untersucht, ist verhältnismässig umfassend, wie ich in Unterunterabschnitt $2.3 .1 \mathrm{zu}-$ sammenfasse. Anders die Situation mit Präferenzen von Medien, also von Journalistinnen und Journalisten, im Kontext parlamentarischen Handelns, also im Kontext der Parlamentsberichterstattung: Hier ist gegenwärtig keine relevante Forschung vorhanden. Überhaupt ist Forschung zu Präferenzen von Journalistinnen und Journalisten sehr selten, und jene Studien, welche explizit mit dem Konzept der Präferenzen operieren, z.B. Boczkowski und Peer (2011) und Boczkowski und Mitchelstein (2015), tun dies eher in einem breiten Verständnis von Präferenz und nicht im engeren Sinne zielorientierten Handelns. Der Grund für diesen Mangel an Forschung dürfte in der unterschiedlichen Komplexität der Handlungssysteme von Parlamentarierinnen und Parlamentariern auf der einen und von Journalistinnen und Journalisten auf der anderen Seite liegen. Die Arbeit von Parlamenten ist an und für sich natürlich ausgesprochen komplex, aber ein bestimmter Teil ist für die Analyse von Präferenzen gut geeignet: Das Abstimmungsverhalten im Parlament. Abstimmungen bedeuten klare Entscheidungsmomente mit nur wenigen Handlungsoptionen (Ja, Nein, Enthaltung, Abwesenheit), und diese Entscheidungsmomente sind oftmals sehr gut dokumentiert und können entsprechend verhältnismässig einfach analysiert werden. Das Handeln von Journalistinnen und Journalisten im Rahmen ihrer Berichterstattung über das Parlament weist keine für eine eingängige Analyse derart zugängliche Handlungskomponente wie Abstimmungen auf. Präferenzen von Medienschaffenden können im Wesentlichen nur durch die sorgfältige inhaltliche Untersuchung des journalistischen Outputs sowie im direkten Gespräch ${ }^{18}$ mit oder Beobachtung von individuellen Journalistinnen und Journalisten eruiert werden, was ungleich aufwendiger ist als die Analyse von Abstimmungsverhalten. Ein anderer Grund für die Abwesenheit von Forschung zu Präferenzen von Medienschaffenden im Kontext parlamentarischen Handelns dürfte dem zwar imaginierten, aber operativ wirksamen Graben zwischen Politikwissenschaft und Kommunika-

18 Als «Gespräch» meine ich hier jede Form der Befragung. 
tionswissenschaft zu verdanken sein. Wie oben im Unterunterabschnitt 2.3.1 deutlich wird, spielen Medien in den engeren Überlegungen zu den Präferenzen von Parlamentarierinnen und Parlamentariern keinerlei Rolle.

Forschung zu Präferenzen von Journalistinnen und Journalisten im Zusammenhang mit Parlamentsberichterstattung im breiteren Sinn ist zwar nicht inexistent, aber dennoch eher selten. Für den Schweizer Kontext ist die frühe Studie von Saxer (Saxer und Hofer 1992, S. 122) wichtig, in welcher festgestellt wird, dass die befragten Journalistinnen und Journalisten, ähnlich wie die Parlamentarierinnen und Parlamentarier, einen Einfluss ihrerseits auf die Arbeit des Parlamentes wahrnehmen. Diesen Einfluss schätzen sie allerdings als deutlich geringer ein als die Parlamentarierinnen und Parlamentarier, denn sie vermuten praktisch keinen direkten Einfluss und nur einen schwachen Einfluss über die Wahrnehmung der Berichterstattung durch Parlamentarierinnen und Parlamentarier sowie über die vermutete Wahrnehmung der Berichterstattung durch die breitere Öffentlichkeit.

Studien, welche sich dem Zusammenhang von Parlament und Medien widmen, behandeln Medienberichterstattung in der Regel in der Logik einer unabhängigen Variable. Das bedeutet, dass im Fokus der Forschung das Handeln von Politikerinnen und Politikern steht, und Medienberichterstattung wird als externer Faktor betrachtet, welcher das Handeln der politischen Akteure beeinflusst und abbildet. Medien werden in solchen Forschungsanordnungen damit indirekt als «Black Box» modelliert, was ein epistemisches Problem darstellen kann:

- In Unterunterabschnitt 2.2.2 beschreibe ich, dass sich die bestehende Literatur mit den Konzepten von «Politiklogik» und «Medienlogik» schwer tut (und schlage darum das Verständnis von Politiklogik und Medienlogik als Präferenzfamilien im Lichte einer Prinzipal-Agent-Beziehung vor). Wie Landerer (2013) beschreibt, ist das Konzept der Medienlogiken in der bestehenden Literatur dominant und das Konzept der Politiklogiken untervertreten.

- In der bestehenden Literatur zum Zusammenhang von Parlament und Medien werden aber nur Parlamentarierinnen und Parlamentariern explizit handlungstheoretisch konzipiert. Medien als Journalistinnen und Journalisten werden als «Black Box» ohne direkten Akteursstatus behandelt.

- Diese Konfiguration mündet darin, dass nur Parlamentarierinnen und Parlamentariern als Akteure behandelt werden, ihr Handeln handlungstheoretisch aber nur als durch Medienlogik bedingt konzipiert wird.

Diese Problemdefinition ist ein wenig zugespitzt, doch sie zeigt auf, warum auch Journalistinnen und Journalisten explizit handlungstheoretisch konzipiert werden sollten. Es gibt im 
Themenbereich der Wechselwirkungen zwischen Parlament und Medien denn auch Studien, die eben diesen konzeptuellen und forschungsprogrammatischen Schritt machen. Die in Unterunterabschnitt 2.3.1 erwähnten Studien zu parlamentarischen Fragestunden (Van Aelst und Vliegenthart 2014; Van Santen, Helfer und Van Aelst 2013; Walgrave und Vliegenhart 2011) operieren zumindest implizit mit handlungstheoretischen Annahmen über Medien. Einer der Befunde in diesen Untersuchungen ist nämlich, dass Medien über jene Fragen in Fragestunden berichten, welche thematisch mit den allgemeinen Themenschwerpunkten der Medien stärker übereinstimmen. Dieser Nebenbefund der Studien ist handlungstheoretisch sehr relevant, da er empirisch Präferenzordnungen nachzeichnet. Die meisten Studien behandeln Medien im Zusammenhang mit Parlamentsberichterstattung aber als «Black Box», so auch Studien mit Bezug zur Schweiz. Beispielsweise widmet sich Tresch (2009) ganz explizit der Frage, wie Medien auswählen, über welche individuellen Parlamentarierinnen und Parlamenterier sie berichten. Obwohl im Zentrum der Fragestellung das Handeln von Journalistinnen und Journalisten steht, behandelt die Autorin Medien konzeptuell als passive «Black Box» und die «Determinanten» der Medienpräsenz sieht die Autorin in Nachrichtenwerten, nach welchen sich Medien richteten, und, welche Parlamentarierinnen und Parlamentarier mehr oder weniger erfüllten. In Unterunterabschnitt 2.2.2 argumentiere ich, dass die Idee von Nachrichtenwerten impliziert, dass Medien als Journalistinnen und Journalisten keine handelnden Akteure sind und, dass Berichterstattung nicht kontingent, sondern durch universale Nachrichtenwerte determiniert ist. In Tat und Wahrheit hat das Konzept der Nachrichtenwerte hier keinen Erklärungsgehalt, denn es wird lediglich etwas, was empirisch beobachtet wird - Medien berichten stärker über Parlamentarierinnen und Parlamentarier in Führungsfunktionen - als «Nachrichtenwert» etikettiert. Ein solches «Black Box»-Verständnis von Medien liegt auch anderen Studien mit Bezug zur Schweiz zugrunde, beispielsweise Kriesi und Hänggli (2012) und Hänggli (2012).

\subsubsection{Wandel der Präferenzen von Parlament und Medien}

Bei der Frage, wie sich die Präferenzen von Parlamentarierinnen und Parlamentariern auf der einen und von Journalistinnen und Journalisten auf der anderen Seite wandeln, und, ob es sich um Wandel im engeren Sinn (aktuelle Medienkrise) oder im breiten Sinn (Wandel über die aktuelle Medienkrise hinaus) handelt, ist die Forschungslage sehr uneben. Darum ist der Forschungsstand für die unterschiedlichen Konfigurationen von Präferenzen und Wandeln zunächst schematisch in Tabelle 1 zusammengefasst.

Die Forschung, welche sich dem Wandel von Präferenzen von Parlamentarierinnen und Parlamentariern im breiteren Sinn im Zuge der Medienkrise widmet, ist überschaubar. Jost, Sülflow 
Tabelle 1: Forschungsstand zu den Präferenzen von Parlament und Medien mit Bezug zur Medienkrise und zu allgemeinem Medienwandel.

\begin{tabular}{lllll}
\hline & Präferenzen Parlament & Präferenzen Medien \\
\hline & eng & breit & eng & breit \\
Medienkrise & $x$ & $\checkmark$ & $x$ & $x$ \\
Allgemeiner Wandel & $x$ & $\checkmark$ & $x$ & $\checkmark$ \\
\hline
\end{tabular}

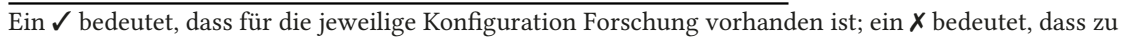
der jeweiligen Konfiguration keine Forschung vorhanden ist. Die Präferenzen der Medien beziehen sich nur auf die Präferenzen von Journalistinnen und Journalisten im Zusammenhang mit Parlamentsberichterstattung.

und Maurer (2015) untersuchen, wie sich die Generaldebatten des deutschen Bundestages von 2000 bis 2010 verändert haben, und, wie Medien über die Generaldebatten berichten. Die Autoren stellen fest, dass die Reden der Oppositionsführer tendenziell negativer und die Reden der Regierung sowie der Oppositionsführer tendenziell personalisierter werden. Allerdings habe nur die Negativität einen relevanten Einfluss auf die Berichterstattung; negativere Inhalte würden eher von den Medien übernommen. Diese Studie ist aber in mehrfacher Hinsicht nicht unproblematisch. So findet der Vergleich zwischen Generaldebatten und Berichterstattung nicht in Form einer Zeitreihe statt, womit letztlich unklar bleibt, ob der Effekt der Negativität durch die Zunahme der Negativität bedingt ist, oder, ob dieser Effekt über die Zeit stabil bleibt. Zudem operiert auch diese Studie mit dem «Black Box»-Modell von Medien und damit verbunden mit dem Konzept von Nachrichtenwerten. Obschon ein deutlicher Befund dieser Studie ist, dass die Nachrichtenwerttheorie in dieser Studie offenbar nicht funktioniert (der Nachrichtenwert der Personalisierung zeitigt keine relevanten Effekte), hinterfragen die Autoren diesen Ansatz nicht.

Der allgemeine, nicht auf die Medienkrise bezogene Wandel der breiten Präferenzen von Parlamentarierinnen und Parlamentariern ist in der Literatur umfassender behandelt. Eine wichtige Entwicklung in den USA ist der Beginn des Service public-Fernsehsenders «C-SPAN» ${ }^{19}$ im Jahr 1979. C-SPAN widmet sich nicht nur, aber in grossen Umfang auch dem US-Kongress, und mit C-SPAN hat sich die öffentliche Wahrnehmbarkeit des Kongresses dramatisch verändert (Frantzich und Sullivan 1996, S. 255-328). C-SPAN berichtet nicht nur selber über den KongressBetrieb, sondern hat seit den Anfängen auch die Funktion eines grossen Reservoirs an Filmund Tonmaterial rund um den Kongress, welches von anderen Medien genutzt werden kann. Der grosse Öffentlichkeitsgewinn des Parlamentes durch C-SPAN hat denn auch bei einigen

19 Eine Abkürzung für «Cable-Satellite Public Affairs Network». 
Forschenden die Frage aufgeworfen, ob sich das Handeln der Kongressabgeordneten durch C-SPAN verändert hat. Die Befunde sind nicht ganz einheitlich, deuten aber darauf, dass die Medienbedachtheit der Kongressabgeordneten sich vor allem in den 1980er Jahren verstärkt hat, ohne aber die Arbeitsweise des Kongresses fundamental zu verändern (T. E. Cook 1986; A. J. Taylor 1998). Im Kontext des allgemeinen Wandels in Deutschland haben Vowe und Dohle (2009) anhand von Stichproben von 1954 bis 2004 untersucht, wie sich u.a. die Anzahl der Medienbezüge in den Bundestagsdebatten verändert. Der Höhepunkt der Medienbezüge (der expliziten Verweise auf Medien innerhalb einer Bundestagsdebatte) war in den späten 1970er Jahren, die generelle Entwicklung ist leicht zyklisch. Tendenziell steigt die Anzahl Medienbezüge aber zwischen 1954 und 2004, wohl aber nicht in einem solchen Masse, dass von einer starken Veränderung der Präferenzen bei den Bundestagsabgeordneten die Rede sein könnte. Allerdings argumentiert auch Marschall $(2001,2009)$ - wenn auch eher anekdotisch und weniger systematisch als Vowe und Dohle (2009) - , dass das deutsche Parlament sich mit der Zeit stärker den Medienlogiken angepasst habe. Ein ähnliches Bild zeichnen ElmelundPræstekær, Hopmann und Nørgaard (2011) für Dänemark. In einem Vergleich zweier Umfragen von 1980 und 2000 zeigt sich, dass dänische Parlamentarierinnen und Parlamentarier im Jahr 2000 in mehrfacher Hinsicht stärker auf Medien bedacht sind (obwohl ein Vergleich nur zweier Zeitpunkte, 1980 und 2000, grundsätzlich auch zufällige Ergebnisse produzieren kann.).

Studien, welche den allgemeinen Wandel der Präferenzen von Journalistinnen und Journalisten im Kontext von Parlamentsberichterstattung untersuchen, sind selten. Ettinger (2013) untersucht den Wandel der Parlamentsberichterstattung in Schweizer Zeitungen anhand von Stichproben zwischen 1962 und 2011. Die Ergebnisse legen nahe, dass sich die Schwerpunkte der Parlamentsberichterstattung gewandelt haben. Mit der Zeit erfolgt die Berichterstattung über Parlamentarierinnen und Parlamentarier zunehmend in Kontexten jenseits des direkten Parlamentsgeschehens (Sessionen und Kommissionsarbeit). Zudem wandelt sich mit der Zeit das Verhältnis der Berichterstattung über das Parlament und über die Exekutive, sprich über den Bundesrat und die Verwaltung: Die Exekutivberichterstattung nimmt zuungunsten der Parlmentsberichterstattung zu. Diese Entwicklungen sieht der Autor als durch den «neuen Strukturwandel der Öffentlichkeit» Imhof (2006b) verursacht. Der neue Strukturwandel postuliert, im Wesentlichen, eine Kommerzialisierung der Medien infolge der gesellschaftlichen Ausdifferenzierung und damit einhergehend einen Wandel der Medienlogiken hin zu grösserer Bindung von Aufmerksamkeit des Publikums durch Themenselektion, -interpretation und -darstellung, welche tendenziell stärkeres Gewicht auf Personalisierung, Skandalisierung, Emotionalisierung und Konfliktinszenierung legt. 


\subsubsection{Fazit und Beitrag der vorliegenden Arbeit}

Die Ausführungen zum Forschungsstand hinsichtlich der drei in dieser Arbeit zu untersuchenden Forschungsfragen zeigen Unterschiedliches auf. Eine erste wichtige Erkenntnis ist, dass Forschung zur Frage von Präferenzen im engeren handlungstheoretischen Sinn bisher mehrheitlich für den US-amerikanischen Kontext stattfindet, und zwar ausschliesslich für die Frage des Abstimmungsverhaltens US-amerikanischer Parlamentarierinnen und Parlamentarier - Journalistinnen und Journalisten als zusätzlich relevante Akteure mit eigenen Präferenzen sind in dieser Forschungstradition inexistent.

Eine zweite wichtige Erkenntnis aus der bestehenden relevanten Literatur ist, dass jene Forschung, welche das Verhältnis von Parlament und Medien untersucht, nicht mit solchen theoretischen Modellen operiert, welche explizit nach Motivstrukturen der Akteure, also nach ihren Präferenzordnungen fragen. Eine solche handlungstheoretische Komponente dringt bei einigen Studien impliziterweise durch, ohne aber explizit und a priori ausformuliert zu sein.

Eine dritte Erkenntnis aus der bestehenden Literatur ist, dass Forschung, welche das Verhältnis von Parlament und Medien untersucht, nur zu einem kleinen Teil den Wandel dieses Verhältnisses über die Zeit untersucht. Jene wenigen Studien, welche das Verhältnis von Parlament und Medien anhand der wechselseitigen inhaltlichen Beeinflussung über einen längeren Zeitraum untersuchen, können, auf den ersten Blick paradoxerweise, keine Aussagen über Veränderungen machen. Bei quantitativen Zeitreheanalysen wird nämlich gemessen, ob über die Zeitreihe hinweg stabil bleibende Effekte bestehen oder nicht; ob sich Effekte über die Zeit verändern, wird damit nicht erfasst.

Eine vierte Erkenntnis aus der bestehenden Literatur ist, dass Forschung rund um das Parlament und um Medien für die Schweiz verhältnismässig umfangreich ist. Diese Erkenntnis mag ein Stück weit dem Umstand geschuldet sein, dass ich auf die Schweiz bezogene Literatur intensiver gesucht habe als sonstige Literatur. Wie dem auch sei, verhältnismässig umfangreiche und vor allem verhältnismässig jüngere Literatur zu diesem Themengebiet mit Schweizer Bezug ist vorhanden. Das bedeutet, dass ich mit der vorliegenden Arbeit weder vollkommen explorative Forschung betreibe, noch, dass ich einen vergessen gegangenen Forschungsbereich wiederbelebe.

Die wesentliche Erkenntnis aus der bestehenden Literatur ergibt sich erst in der Summe der bisher genannten Punkte. Forschung rund um die Frage der Präferenzen von Parlament und Medien in einem engeren und in einem breiteren Sinn ist vorhanden, und zwar nicht zu knapp. Allerdings handelt es sich dabei um eher isolierte Forschungsprogramme, welche in erster Linie durch eine gewisse Schnittmenge in Betreff des Forschungsgegenstandes mit- 
einander verbunden sind. Die zentralen Fragestellungen der vorliegenden Arbeit, und damit ihre theoretisch-normative Herleitung, ist so bisher noch nicht behandelt. Das Ziel der vorliegenden Arbeit ist es denn auch, diese Lücke zu füllen. Die Forschungslücke umfasst folgende Komponenten im Sinne der in Unterunterabschnitt 2.2.3 formulierten Forschungsfragen:

- Die normative Ausgangslage der Untersuchung ist die prozeduralisierte Volkssouveränität als Prinzipal-Agent-Beziehung zwischen den Bürgerinnen und Bürgern (Prinzipal) und den Parlamentarierinnen und Parlamentariern (Agent).

- Die empirischen Fragestellungen fokussieren auf das Handeln der Parlamentarierinnen und Parlamentarier sowie der Journalistinnen und Journalisten im Kontext öffentlicher Kommunikation über das Parlament.

- Politiklogiken und Medienlogiken werden konzeptuell als zwei Präferenzfamilien im Rahmen parlamentarischen Handelns vorgeschlagen.

- Ziel der Arbeit ist es, zu analysieren, in welchem Masse die Akteure im Parlament und in den Medien nach den Politiklogiken und in welchem Masse nach den Medienlogiken handeln.

- Ein weiteres Ziel besteht darin, in Erfahrung zu bringen, ob sich das Handeln der Akteure im Parlament und in den Medien im Zuge der Medienkrise verändert.

Bisher existieren keine Studien, welche diese Komponenten in einer Untersuchung miteinander verweben. In diesem Zusammenhang muss an dieser Stelle bereits auf einen Aspekt des Forschungsdesigns vorgegriffen werden (das Forschungsdesign ist genauer in Abschnitt 4 besprochen), da ich die Designentscheidungen auch angesichts der bestehenden Literatur treffe. Die erste Designentscheidung betrifft die Frage, welcher Teil der Bundesversammlung warum untersucht werden soll. In Unterunterabschnitt 2.3.1 kritisiere ich Forschung, welche teils ohne gute Begründungen einen Teil des Parlamentes untersucht und die Ergebnisse pars pro toto generalisiert. Ein solches Vorgehen bedeutet in der Regel, dass der forschungsökonomisch am einfachsten zu untersuchende Teil des Parlamentes analysiert wird, mit der Ungewissheit, ob dieser untersuchte Teil ein genaues Bild der allgemeinen Vorgänge zeichnet. In dieser Arbeit fokussiere ich nur auf einen Aspekt der Bundesversammlung, im Wissen, dass andere Aspekte womöglich anders funktionieren: Die Sessionen. Die Schweizer Bundesversammlung als Milizparlament ${ }^{20}$ tagt nicht ganzjährig, sondern in vier regulären Sessionen à drei Wochen und

20 Ob die Bezeichnung «Milizparlament» für die Bundesversammlung angesichts des professionalisierten Parlamentsbetriebes überhaupt noch Sinn macht, ist unklar (Bütikofer 2014, S. 58-90). Normativ gesehen muss eine Professionalisierung des Milizparlamentes aber nicht problematisch sein. Wie bereits M. Weber (1919, S. 10-13) bemerkt, hat Politik als «Nebenberuf» zumindest historisch recht eigentlich wenig mit Basisdemokratie zu tun und mehr mit den Partikularinteressen potenter Gruppen. 
optional in je einer einwöchigen Sondersession pro Kammer. Zwei anderen bedeutenden Phasen im Parlamentskontext widme ich mich nicht bzw. nur am Rande: Der Kommissionsarbeit sowie den Wahlen.

Open Access Dieses Buch wird unter der Creative Commons Namensnennung - Nicht kommerziell 4.0 International Lizenz (http://creativecommons.org/licenses/by-nc/4.0/deed. de) veröffentlicht, welche für nicht kommerzielle Zwecke die Nutzung, Vervielfältigung, Bearbeitung, Verbreitung und Wiedergabe in jeglichem Medium und Format erlaubt, sofern Sie den/die ursprünglichen Autor(en) und die Quelle ordnungsgemäß nennen, einen Link zur Creative Commons Lizenz beifügen und angeben, ob Änderungen vorgenommen wurden.

Etwaige Abbildungen oder sonstiges Drittmaterial unterliegen ebenfalls der genannten Creative Commons Lizenz, sofern sich aus der Abbildungslegende oder der Quellreferenz nichts anderes ergibt. Sofern solches Drittmaterial nicht unter der genannten Creative Commons Lizenz steht, ist eine Vervielfältigung, Bearbeitung oder öffentliche Wiedergabe nur mit vorheriger Zustimmung des betreffenden Rechteinhabers oder auf der Grundlage einschlägiger gesetzlicher Erlaubnisvorschriften zulässig.

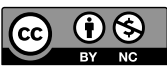




\section{Theoretische Einbettung}

\subsection{Agenda Setting}

Agenda Setting ist ein zentraler Ansatz der Kommunikationswissenschaft (Bryant und Miron 2004). Die Attraktivität dieses Ansatzes liegt vermutlich nicht zuletzt in dessen zeitloser Eleganz, denn die elementare Idee von Agenda Setting ist seit der wegweisenden Studie von McCombs und Shaw (1972) nach wie vor unverändert: In einem bestimmten Teilsystem werden bestimmte Themen unterschiedlich häufig behandelt, und diese relative Wichtigkeit von Themen (oftmals als «Salienz», im Englischen «salience», beschrieben) in einem Teilsystem hat einen Effekt auf die Wahrnehmung der relativen Wichtigkeit der Themen in einem anderen Teilsystem. Ein Grossteil der Agenda Setting-Forschung interessiert sich dabei für das Verhältnis zweier Teilsysteme, jenes der Medien und jenes der Öffentlichkeit im Sinne der individuellen Bürgerinnen und Bürger (McCombs 2004, S. 36-52, 2005). Bei dem Verhältnis dieser zwei Teilsysteme wird in erster Linie eine Wirkungsrichtung untersucht, nämlich der allfällige Einfluss der Agenda der Medien auf die Agenda der Bürgerinnen und Bürger; sprich, ob die Themenprioritäten der Medien die Themenprioritäten bei den Bürgerinnen und Bürgern beeinflussen. Dieser Agenda Setting-Effekt von den Medien auf individuelle Bürgerinnen und Bürger wurde in Hunderten von Studien geprüft, und obschon die Kontexte und konkreten empirischen Vorgehensweisen in keinen zwei Studien identisch sind, kann davon ausgegangen werden, dass dieser Agenda Setting-Effekt existiert (Wanta und Ghanem 2006).

Der Agenda Setting-Ansatz hat eine Reihe von Erweiterungen und Weiterentwicklungen erfahren. Eine davon ist die Überlegung, dass Agenda Setting nicht nur bedeutet, worüber wir denken, sondern auch, wie wir darüber denken. Die Annahme, dass Agenda Setting nicht nur das Was, sondern auch das Wie betrifft, ist als «Second Level»-Agenda Setting beschrieben (Golan und Wanta 2001; McCombs, Llamas und Rey 1997). «Second Level»-Agenda Setting ist auch unter dem Begriff «Attribute»-Agenda Setting bekannt (Kim, Scheufele und Shanahan 2002; McCombs, Llamas und Rey 1997); die Verwendung der Begriffe ist synonym. Dass Medien nicht nur beeinflussen, was für Themen wir als wichtig erachten, sondern auch, wie wir über diese Themen denken, ist besonders für Wahlkämpfe gut beforscht. Ein berühmtes Beispiel für diesen Effekt ist die Debatte zwischen John F. Kennedy und Richard Nixon im Jahr 1960. Personen, welche die Debatte im Radio verfolgt hatten, waren eher der Meinung, Nixon habe besser debattiert. Personen, welche die Debatte im Fernsehen verfolgt haben, hatten hingegen einen schlechteren Eindruck von Nixon (A. A. Cohen 1976); dieser spezifische Effekt liess sich in einem Versuch viele Jahre nach der Debatte rekonstruieren (Druckman 2003). Das Beispiel 
der Nixon-Kennedy-Debatte verdeutlicht, dass Berichterstattung, dass also journalistisches Handeln, immer ein Vorgang der selektiven Aufnahme, Verarbeitung und Darstellung von Informationen zum Zwecke der intersubjektiven Bedeutungsherstellung ist. Darum ist es unumgänglich, dass nicht nur ein Was, sondern auch ein Wie im Zuge der Berichterstattung kommuniziert wird.

Das Wie steht auch im Zentrum des «Framing»-Ansatzes, welcher den Fokus auf die Frage legt, wie genau die Inhalte der Berichterstattung kontextualisiert werden (Entman 1993). Der Framing-Ansatz ist nicht aus dem Agenda Setting-Ansatz entstanden, sondern hat sich, mehr oder weniger, parallel zu diesem entwickelt, und ihn in seiner Beliebtheit überholt, um das wohl dominanteste Konzept innerhalb der Kommunikationswissenschaft zu werden (D. H. Weaver 2007). Die genaue Genese des Framing-Ansatzes innerhalb der Kommunikationswissenschaften ist dabei unklar. Historisch werden bisweilen der sozialkonstruktivistische Ansatz nach Goffman und Berger (1986) und der kognitionspsychologische Ansatz nach Tversky und Kahneman (1986) als Ursprünge des kommunikationswissenschaftlichen Framing-Ansatzes verstanden, doch trotz des jeweils geteilten Begriffes «Frame» ist eine solche Genealogie unsicher. Framing bezieht sich in der kommunikationswissenschaftlichen Praxis nämlich nicht so sehr auf die kognitiven Effekte unterschiedlicher «Verpackungen» von Berichterstattung bei Individuen, sondern vor allem auf die inhaltliche Ausgestaltung der Berichterstattung selber (Reese 2007; Scheufele und Tewksbury 2007). Wie genau die Herkunft des Framing-Ansatzes auch aussehen mag, dessen Zukunft scheint in der Verschmelzung mit Agenda Setting, vor allem mit AttributeAgenda Setting zu liegen (McCombs und Ghanem 2001), da sich diese zwei Ansätze im Grunde für dasselbe Phänomen interessieren (das Wie der Berichterstattung), scherpunktmässig aber auf unterschiedliche Aspekte davon abzielen (Effekte bei Individuen bei Agenda Setting und die konkrete Herstellung des Wie innerhalb der Berichterstattung bei Framing).

Eine dritte Weiterentwicklung im Kontext von Agenda Setting ist der sogenannten «Priming»-Ansatz. Der Priming-Ansatz geht davon aus, dass Berichterstattung nicht nur die subjektive Wichtigkeit eines Themas beeinflusst, sondern darüber hinaus auch die subjektiv wahrgenommene Bedeutung von Kriterien, nach denen Politikerinnen und Politiker (Iyengar und A. Simon 1993; Sheafer und Weimann 2005) oder auch politische Inhalte (Kim, Han u. a. 2012) beurteilt werden. Ähnlich wie bei Framing lässt sich die genaue Entstehung des Priming-Ansatzes innerhalb der Kommunikationswissenschaft nicht genau nachzeichnen. Der Priming-Effekt als psychologisches Phänomen beschreibt den Umstand, dass der Kontakt mit einem Reiz die Reaktion auf einen anderen Reiz beeinflussen kann (D. E. Meyer und Schvaneveldt 1971; D. E. Meyer, Schvaneveldt und Ruddy 1974; Schvaneveldt, D. E. Meyer und Becker 1976; 
Tulving und Schacter 1990). Ähnlich wie bei Framing ist hier nicht ohne Weiteres klar, wie die Brücke von dieser psychologischen Forschung zu den kommunikationswissenschaftlichen Konzepten geschlagen wird - es handelt sich in beiden Fällen wohl zumindest ein Stück weit um metaphorische und weniger um konkrete inhaltliche Brücken.

In der kommunikationswissenschaftlichen Forschung mögen die Weiterentwicklungen des Agenda Setting-Ansatzes mittlerweile prominenter vertreten sein als als einfaches Agenda Setting, aber ich stütze mich in der vorliegenden Arbeit trotzdem auf das einfache Agenda Setting. Einer der Gründe dafür ist die nach meinem Dafürhalten solidere Plausibilität der Minimalvariante des Ansatzes. Während Agenda Setting auf individueller Ebene gut mit kognitionspsychologischen Mechanismen wie etwa der «Availability»-Heuristik (N. Schwarz u. a. 1991; Tversky und Kahneman 1973) und dem «Mere Exposure»-Effekt (Zajonc 1968, 2001) unterfüttert werden kann, sind die Grundlagen von Attribute-Agenda Setting, Framing und Priming weniger gesichert.

\subsubsection{Agenda Setting und die Politikagenda}

Das Forschungsinteresse bei Agenda Setting-Fragestellungen liegt oftmals, wie oben argumentiert, in der Beziehung der Agenda der Medien zu der Agenda der individuellen Bürgerinnen und Bürger. Für die vorliegende Arbeit ist aber ein anderer, etwas weniger umfangreich beforschter Zusammenhang von Belang: Die Beziehung der Agenda der Medien mit der Agenda des Parlamentes. Forschung zur Agenda des Parlamentes im Besonderen und der Agenda staatlicher Akteure im Allgemeinen hat vor allem in politikwissenschaftlichem Kontext Tradition, etwa bei Baumgartner und Jones (2010), Jones und Baumgartner (2005) und Kingdon (2010). In diesem Verständnis wird die Politikagenda als System konzipiert, welches Reize aus der Umwelt, darunter auch den Medien, wahrnimmt und je nach Reizschwellen reagiert. Die politikwissenschaftliche Agenda Setting-Forschung operiert dabei unter dem «Punctuated Equilibrium»-Paradigma (Baumgartner, Jones und Mortensen 2014), welches besagt, dass Wandel politischer Inhalte, also politsicher Agenden, sehr langsam und träge ist, punktuell aber starke Umbruchphasen aufweisen kann. Ein zentraler Befund der politikwissenschaftlichen Forschungstradition ist, dass politsche Systeme ihre Agenden im Sinne der «Punctuated Equilibrium»-These empirisch nach dem «Feast or Famine»-Muster wandeln - meistens passiert sehr wenig bis nichts, und selten passiert viel. Die politikwissenschaftliche Forschungstradition liefert wertvolle Erkenntnisse, aber es handelt sich bei dieser Perspektive um ein rein systemtheoretisches Denken ohne handlungstheoretische Komponente. Die Politikagenda komplexitätsreduziert als «Black Box» zu konzipieren, welche auf Reize reagiert, liefert auf einer abstrakten Ebene relevante 
Erkenntnisse, aber ein solches Vorgehen alleine hat noch keinerlei Erklärungsgehalt. Darum bediene ich mich für diese Arbeit eines stärker kommunikationswissenschaftlich, oder, genauer gesagt, interdisziplinär-handlungstheoretischen Verständnisses von Agenda Setting zwischen Medien und Parlament.

Die klassische Agenda Setting-Forschung untersucht grossteils eine Wirkungsrichtung, nämlich den Einfluss der Medien auf individuelle Bürgerinnen und Bürger. Aus meinen bisherigen Ausführungen insbesondere in Abschnitt 2 rund um den Entdeckungszusammenhang wird aber ersichtlich, dass im Lichte der Fragestellungen nicht nur von Interesse ist, ob und wie Medien das Parlament beeinflussen, sondern auch umgekehrt, ob und wie das Parlament die Medien beeinflusst. Die Frage, wie die Agenda der Medien zustande kommt, wird in der Agenda Setting-Literatur als «Agenda Building» beschrieben (D. Weaver und Elliott 1985; Wirth u. a. 2010). Die Unterscheidung nach Agenda Setting und Agenda Building ist auf der sprachlichen Ebene etwas unglücklich. In kommunikationswissenschaftlichem Kontext meint Agenda Building das Herstellen der Medienagenda (Denham 2010). Es ist konzeptuell allerdings wenig schlüssig, den Vorgang der Agenda-Herstellung bei individuellen Bürgerinnen und Bürgern wie auch bei politischen Akteuren als «Setzen» («Setting») zu beschreiben, bei Medien hingegen als «Erstellen» («building») - diese unnötig unterschiedliche Terminologie ist eher, so meine Vermutung, disziplinärem Grabendenken zu verdanken denn konzeptuellen Notwendigkeiten. Darum verzichte ich auf die sprachliche Unterscheidung nach Agenda Setting und Agenda Building und verwende der konzeptuellen Einfachheit wegen stattdessen universal den Begriff Agenda Setting.

\subsubsection{Theorie oder proto-theoretisches Framework?}

Bisher habe ich bewusst von Agenda Setting als Ansatz und nicht von Agenda Setting als Theorie gesprochen. Damit will ich nicht signalisieren, dass Agenda Setting grundsätzlich keine Theorie sei. Wenn eine wissenschaftliche Theorie als Menge von Sätzen, oder, zeitgemässer, als Familie von Modellen verstanden wird (Chakravartty 2001; Suppe 2000) und Modelle bedeuten, dass aus den generellen Prämissen der Theorie Formulierungen erstellt werden, deren Elemente einen oder mehrere Aspekte der Welt repräsentieren (Giere 2004), dann hat Agenda Setting grundsätzlich die Qualität einer Theorie. Zwar gibt es im Agenda SettingAnsatz strittige Punkte (Takeshita 2005), aber diese gibt es in allen wissenschaftlichen Theorien. Das potenzielle Problem ist eher, dass die Familie an Modellen, welche sich aus der Agenda Setting-Theorie ableiten lassen, in erster Linie auf die klassische Frage des Effektes der Medien auf individuelle Bürgerinnen und Bürger gerichtet ist. Aus den sozialkonstruktivistischen 
und kognitionspsychologischen Prämissen der Agenda Setting-Theorie für die individuelle Mikro-Ebene ergeben sich keine Modelle, welche die Wechselwirkungen zwischen Medien und politischen Akteuren im Allgemeinen, dem Parlament im Besonderen beschreiben. Zwar ist es möglich, politische Akteure einfach als eine weitere Instanz der Mikro-Ebene von Bürgerinnen und Bürgern zu konzipieren, aber ein solches Modell ist banal. Es ist durchaus wahrscheinlich, dass Individuen, welche das Personal der politischen Akteure bilden, denselben kognitionspsychologischen Effekten unterliegen wie der Rest der Bevölkerung. Allerdings ist aus einer handlungstheoretischen Perspektive nicht anzunehmen, dass nur solche Effekte das Agenda Setting zwischen Medien und politischen Akteuren erklären. Darum besitzt Agenda Setting in Betreff der Beziehungen zwischen Medien und politischen Akteuren keinen wirklichen Theoriecharakter, sondern den Charakter eines proto-theoretischen Gebildes. Erst, wenn der Agenda Setting-Ansatz um plausible, generalisierte Annahmen ob der Effekte zwischen Medien und politischen Akteuren ergänzt wird, erhält der Ansatz auch in dieser Agenden-Konfiguration Theoriecharakter.

Einen wichtigen Schritt in diese Richtung unternehmen Walgrave und Van Aelst (2006). Die Autoren behandeln zwar nicht ganz explizit die Frage des Theoriecharakters von Agenda Setting im Kontext von Medien und Politik, sondern gehen quasi-induktiv vor: Anhand der bestehenden Forschung, welche zwar überschaubar, aber nicht komplett ohne konzeptuelle Überlegungen ist (darum nenne ich das Vorgehen der Autoren quasi-induktiv anstatt rein induktiv), versuchen die Autoren festzuhalten, warum was für Agenda Setting-Effekte zwischen Medien und politischen Akteuren zu erwarten sind. In dieser Studie wird denn auch betont, dass es nötig ist, Agenda Setting zwischen Medien und politischen Akteuren handlungstheoretisch zu konzeptualisieren, da das klassische Erklärungsmodell von Agenda Setting auf passiv-kognitiven Annahmen beruht (ebd., S. 99):

Although it is easy for a member of the public to shift attention - it does not cost a thing because attention is just caring about - shifting attention is a completely different thing for political actors. It entails the laborious and conflictual reallocation of time, personnel, and resources. Precisely for that reason, we need a specific behavioral theory of political actors and we cannot rely on the cognitive public agenda setting model. [Kursiv im Original]

Die Autoren identifizieren fünf Kontextfaktoren, welche Agenda Setting von Medien auf politische Akteure beeinflussen: Die Politikphase (Wahl- vs. Routinephasen), institutionelle Regeln, die interne Arbeitsweise (z.B. Absprachen innerhalb der Partei), die politische Konfiguration 
(Regierung und Opposition) sowie die individuellen Eigenschaften der Politikerinnen und Politiker. Von diesen Kontextfaktoren sind nicht alle gleichermassen geeignet, den Agenda Setting-Ansatz zu einer Theorie anzureichern. Die spezifische politische Konfiguration, die interne Arbeitsweise von Akteuren sowie die Politikphase sind ganz konkrete, fallbezogene Faktoren. Individuelle Eigenschaften und institutionelle Regeln hingegen sind zwei Aspekte, welche sich generalisieren und theoretisch aufladen lassen. Die individuellen Eigenschaften meinen, so mein Argument in Unterabschnitt 3.2, die Präferenzen von Politikerinnen und Politikern, bzw. konkreter, von Parlamentarierinnen und Parlamentariern - es gibt gute Gründe, anzunehmen, dass die Präferenzfamilie der Medienlogiken eine Rolle für sie spielt. Die institutionellen Regeln erklären in einem zweiten Schritt, so mein Argument in Unterabschnitt 3.3, in welchen parlamentarischen Arenen Parlamentarierinnen und Parlamentarier in welchem Masse nach Politiklogiken und in welchem Masse nach Medienlogiken handeln.

\subsection{Medialisierung}

Massenmedien spielen in demokratischen Systemen eine sehr bedeutende Rolle, nicht zuletzt, weil sie aus funktionaler Perspektive eine notwendige Bedingung für die Existenz von Öffentlichkeit sind. Die Rolle der Medien in zeitgenössischen Gesellschaften erschöpft sich aber nicht in einzelnen funktionalen Komponenten. Medien, und damit öffentliche Kommunikation, sind ein Bestandteil zahlreicher Handlungssphären und Teilsysteme der Gesellschaft. Das bedeutet nicht unbedingt, dass Medien immer und überall aktiv «dabei sind»; Medien sind nicht omnipräsent in modernen Gesellschaften. Medien sind aber auf eine solche Art mit der gesamten Gesellschaft verwoben, dass die restlichen gesellschaftlichen Teilsysteme und Handlungssphären Medien und Medienlogiken tendenziell wahrnehmen, und sie ein Stück weit auch in die eigenen Funktionslogiken integrieren. Dieses Phänomen beschreibt Hjarvard (2008, S. 119) folgendermassen:

By the mediatization of society, the process whereby society to an increasing degree is submitted to, or becomes dependent on, the media and their logic. This process is characterized by a duality in that the media have become integrated into the operations of other social institutions, while they also have acquired the status of social institutions in their own right. [Kursiv im Original]

Die Annahme, dass Medien nicht einfach ein abgeschlossenes Teilsystem der Gesellschaft sind, sondern, dass die Logiken der Medien alle Teilsysteme und Handlungssphären derart 
durchdringen, dass Anpassungsleistungen stattfinden, ist der Kern der Theorie der Medialisierung $^{21}$. Anpassungsleistungen an die Logiken der Medien ist zwar die Kernannahme von Medialisierung, doch es gibt keinen abgeschlossenen Katalog an medialisierungsinduzierten Anpassungsleistungen; eher gibt es Versuche, unterschiedliche Dimensionen von Medialisierung zu fassen. Schulz (2004) unterscheidet in diesem Sinne vier Dimensionen von Medialisierung: «Extension» (Medien erweitern die menschlichen Kommunikationspotenziale), «Substitution» (Medien ersetzen Formen «klassischer» sozialer Interaktion), «Amalgamation» (Medien werden Teil von Handlungssphären, in welche sie bisher nicht hineinspielten) und «Accommodation» (Die eigentlichen Anpassungsleistungen an die Medien). «Accommodation» als Anpassungsleistungen beschreibt der Autor anhand des Beispiels politischer Akteure, welche sich den Medienlogiken fügen, um mehr Publizität zu erhalten. Imhof (2006a, S. 207) schlägt aus einer differenzierungstheoretischen Perspektive einen breiteren Katalog konkreter Medialisierungseffekte vor und ordnet diese nach zwei Dimensionen, der gesellschaftlichen Ebene (mikro, meso und makro) sowie der Art der Differenzierung (funktional, stratifikatorisch, segmentär). Dieser umfassende Katalog an Effekten sprengt aber das Medialisierungskonzept. So ist es beispielsweise wenig zweckdienlich, die Zunahme internationaler Politik ${ }^{22}$ in den konzeptionellen Dunstkreis der Medialisierung zu stellen. Eher handelt es sich bei internationaler Politik um ein klassisches Prinzipal-Agent-Problem, welches dadurch verschärft wird, dass die öffentliche Beobachtbarkeit internationaler Akteure gering ist.

Es ist ausgesprochen schwierig, einen umfassenden und gleichzeitig trennscharfen Katalog an Medialisierungseffekten aufzustellen. Darum ist es sinnvoll, Medialisierung als Theorie von konkreten, aber situativen Medialisierungseffekten zu trennen - die konkreten Folgen von Medialisierung müssen nicht für alle empirischen Konstellationen universal identisch sein. Medialisierung ist die theoretische Triebfeder, welche die empirischen Beobachtungen erklärt. Wenn Medialisierung als breites theoretisches Erklärungsmodell nützlich sein soll, dann muss Medialisierung verstanden als Anpassungsleistungen an Medienlogiken nicht nur punktuelle, sondern allgemeine Relevanz haben. Diese allgemeine, gesellschaftsweite Bedeutung von Medialisierung wird bisweilen etwas alarmistisch mit dem Konzept des Totalphänomens

21 Im Deutschen werden sowohl «Medialisierung» wie auch «Mediatisierung» verwendet, um das englische «Mediatization» zu beschreiben. Die zwei deutschen Begriffe erachte ich als synonym und verwende ausschliesslich «Medialisierung».

22 Imhof (2006a, S. 197) beschreibt u.a. die G8, die WTO und das IMF als «transnational». Ich verwende den Begriff «international», weil es im Jargon der Forschung zu internationalen Beziehung üblich ist, multilaterale Politik mit staatlicher Beteiligung als «international» zu bezeichnen. «Transnational» hingegen meint eher solche internationale Politik, welche zivilgesellschaftlichen Charakter hat, also ohne Beteiligung staatlicher oder wirtschaftlicher Akteure ist. Soziale Bewegungen werden als typisches Beispiels für transnationale Politik gehandhabt (Tarrow 2001). 
beschrieben. Ein wichtiger Verfechter des Konzepts der Medialisierung als Totalphänomen, zumindest im deutschsprachigen Raum, ist Saxer (2012, S. 271):

[...] Medialisierung [operiert] dementsprechend als gesellschaftliches Totalphänomen, das alle Dimensionen des sozialen Seins durchwirkt und im Sozietätstyp Mediengesellschaft kulminiert.

Wenn Medialisierung konzeptuell als Totalphänomen gedacht wird, welches «alle Dimensionen des sozialen Seins» durchwirkt, dann werden fast automatisch normative Assoziationen geweckt. Bei der Vorstellung, dass ein Teilsystem, die Medien, mit ihren Logiken alle anderen Teilsysteme beeinflusst, schwingt nämliche eine gewisse zumindest bildliche Konnotation von Übergriff, Belagerung und Invasion mit. Tatsächlich wird Medialisierung im Kontext der politischen Kommunikation oftmals als für Demokratie bestenfalls nicht schädlich, schlimmstensfalls als die Demokratie erodierend beschrieben. So warnen etwa Mazzoleni und Schulz (1999, S. 250):

Mediatized politics is politics that has lost its autonomy, has become dependent in its central functions on mass media, and is continuously shaped by interactions with mass media.

Auch Matthes und Esser (2013, S. 177) konnotieren Medialisierung negativ und beschreiben diese als «Eindringen» von Medienlogiken in Orte, wo diese offenbar nicht hingehören:

We define mediatization as the growing intrusion of media logic as an institutional rule into fields where other rules of defining appropriate behavior prevailed [...].

Eine a priori normativ ablehnende Haltung gegenüber Medialisierung und Medienlogiken ist in mindestens vierfacher Hinsicht problematisch. Erstens gründet eine solche normative Haltung auf der impliziten Prämisse, dass der status quo normativ immer Vorrang vor Veränderung hat. Die Idee, dass Konservatismus immer die bessere Wahl als Veränderung darstellt, ist rational kaum begründbar und hat eher Züge des status quo-Bias (Samuelson und Zeckhauser 1988). Das bedeutet aber auch nicht, dass Medialisierung im Sinne von Anpassungsleistungen immer positiv sein muss. Mir scheint, eine vernünftige Haltung a priori ist, Medialisierung sowohl potenzielle negative wie auch potenzielle positive Effekte zuzusprechen. In Abbildung 4 mache ich dieses Argument explizit für die Präferenzfamilien der Politiklogiken und der Medienlogiken. Wenn im Zuge parlamentarischen Handeln Medienlogiken zum Ausdruck kommen, 
ohne, dass sie eine Schnittmenge mit Politiklogiken haben, erachte ich dies als normativ negativ. Der Vorteil in dem vorgeschlagenen normativen Bezugsrahmen der prozeduralisierten Volkssouveränität als Prinzipal-Agent-Beziehung ist dabei, dass das normative Problem relativ klar beschrieben wird und es nicht nötig ist, sehr umfassende, dafür aber nur leidliche präzise allgemeine «Gefahren» für Demokratie heraufzubeschwören. Im Modell in Abbildung 4 wird zudem ersichtlich, dass ich davon ausgehe, dass es durchaus eine Schnittmenge geben kann zwischen Medienlogiken und Politiklogiken. Diese Schnittmenge ist medialisiertes Handeln, welches aber zugunsten der Präferenzen der Bürgerinnen und Bürger ist. In welchem Masse Medienlogiken zuungunsten der Politiklogiken zum Ausdruck kommen und, in welchem Masse Politiklogiken unter Einbezug der Medienlogiken zum Ausdruck kommen, ist eine Frage der Empirie - und diese Arbeit ein Versuch, diese Frage ein Stück weit zu klären.

Zweitens impliziert eine a priori normativ ablehnende Haltung gegenüber Medialisierung oft, wie Pontzen (2006, S. 11) bemerkt, dass sich Politik den Medienlogiken unterwirft, dass also Medialisierung als ominöser und unaufhaltbarer «Meta-Prozess» (Krotz 2007, S. 38), gegenüber welchem die Politik ohnmächtig ist, die Gesellschaft verändert. Ein solches Verständnis von Täter und Opfer ist dramaturgisch nicht zuletzt darum ansprechend, weil damit auch Metaphern wie «Kolonisierung» (T. Meyer 2001) bedient werden können. Konzeptuell allerdings sind solche Schemata Rückschritte in die Zeit der befürchteten Medienallmacht (Bonfadelli und Friemel 2014, S. 35). Wenn das politische System als aus Akteuren zusammengesetzt gedacht wird - und das ist das dieser Arbeit zugrunde liegende Politikbild - , dann greift ein Verständnis von Medialisierung als blosse passive Reaktion auf exogene Reize zu kurz. Erneut lohnt sich ein Blick auf Abbildung 4 mit den Schnittmengen der Politiklogiken und der Medienlogiken. Es ist möglich, dass medialisiertes Handeln im Kontext des Parlamentes bedeutet, dass Parlamentarierinnen und Parlamentarier nolens volens Handlungszwang ausgesetzt sind. Aber genau so muss es plausiblerweise möglich sein, dass medialisiertes Handeln bedeutet, dass Parlamentarierinnen und Parlamentarier im Sinne der Medienlogiken handeln, um dadurch strategisch die eigenen Ziele zu erreichen (egal, ob das in diesem Beispiel Politiklogiken sind, also die Ziele der Bürgeinnen und Bürger, oder sonstige andere Ziele). Wenn Medialisierung Anpassungsleistungen im (politischen) Handeln bedeutet, dann müssen die Anpassungsleistungen auch den Charakter bewussten, strategischen Handelns haben können.

Drittens befindet sich eine a priori normativ ablehnende Haltung gegenüber Medialisierung nicht in einem agnostischen, also empirisch völlig unbeforschten Raum. Zahlreiche Studien ${ }^{23}$ widmen sich der Suche nach negativen Medialisierungseffekten und kommen zum insgesamt

23 Um nur einige zu nennen: Matthes und Esser (2013), Newton (2006), Reinemann (2010) und Vowe (2006) 
ernüchternden Schluss, dass die befürchteten für Demokratie dysfunktionalen Effekte doch weitgehend ausbleiben. Das bedeutet allerdings nicht, dass Medialisierung nicht tiefgreifende Veränderungen bewirkt. Ein viel diskutiertes Beispiel sind rechtspopulistische Parteien, welche vor allem in Europa nach der Jahrtausendwende grosse Erfolge verzeichneten. Der Aufstieg rechtspopulistischer Parteien kann aus der theoretischen Perspektive der Medialisierung miterklärt werden, denn diese Parteien bedienen die Logiken der Medien in der Regel sehr erfolgreich (Mudde 2007, S. 248-253). Auch wenn rechtspopulistische Parteien als durch Medialisierung mitbedingt verstanden werden, bedeutet das nicht, dass rechtspopulistische Parteien automatisch eine wie auch immer geartete Gefahr für Demokratie darstellen (Mudde 2010).

Viertens, und die drei vorherigen Punkte zusammenfassend: Das Konzept der Medialisierung in der «klassischen» Verwendung als Totalphänomen, welches das politische Handeln fundamental dominiert, ist fast sicher massloss übertrieben (Brants und Praag 2015). Die Idee, dass Medialisierung als Totalphänomen beschrieben werden kann und damit jedes Handeln erklärt, ist eine Art von Denkfehler, die Maslow (1966, S. 15) bildlich folgendermassen beschreibt:

I suppose it is tempting, if the only tool you have is a hammer, to treat everything as if it were a nail.

Verfechter der Medialisierungsthese tendieren also bisweilen, Medialisierung als Pauschalerklärung zu verwenden, inklusive der Idee der «Kolonisierung» und der aufgezwungenen Anpassungsleistungen seitens politischer Akteure. Eine so gedachte allumfassende Medialisierung hat aber letztlich keinen empirischen Gehalt - eine Theorie, die meint, alles zu erklären, erklärt nichts. Das bedeutet allerdings nicht, dass das Konzept der Medialisierung grundsätzlich nutzlos ist, wie ich im Folgenden argumentiere.

\subsubsection{Medialisierung oder Medialisiertheit?}

Der Begriff «Medialisierung» impliziert die Existenz im Mindesten zweier Zustände, eines nichtmedialisierten und eines medialisierten. Medialisierung impliziert also direkt einen Vorgang, und Medialisierung hat bereits auf der begrifflichen Ebene Prozesscharakter. Der implizierte Prozesscharakter im Begriff der Medialisierung ist nicht bloss ein sprachlicher Zufall des Deutschen; auch in dem englischen Originalbegriff für Medialisierung, «Mediatization», schwingt der Prozesscharakter mehr als nur konnotativ mit. Wenn Medialisierung als Prozess verstanden wird, dann stellt sich die Frage, wie genau dieser Prozess aussieht. Die Medialisierungsliteratur adressiert diese Frage, eigentmülicherweise, nicht in einem Masse, wie das angesichts des einen 
Prozess andeutenden Begriffes Medialisierung zu erwarten wäre. In einem viel beachteten Aufsatz zu dieser Problematik bemerkt Strömbäck (2008, S. 229) denn auch:

Moreover, there is a lack of analysis of the process of mediatization, although the concept as such denotes a process.

In dieser Studie unternimmt Strömbäck den Versuch, Medialisierung historisch zu verorten, und zwar für Westeuropa und für die Zeit nach dem zweiten Weltkrieg. Er identifiziert vier Phasen. In der ersten Phase werden Medien zur wichtigsten Informationsquelle für Bürgerinnen und Bürger in politischen Belangen. In der zweiten Phase emanzipieren sich die Medien vom politischen System und handeln nach Medienlogiken anstatt nach Politiklogiken. In der dritten Phase entwickeln sich die Medien stärker weiter in ihrer Unabhängigkeit und werden noch autonomer; so autonom, dass politische Akteure sich anpassen müssen, um das gewünschte Gut, Publizität, zu erhalten. In der vierten Phase wandeln sich die Anpassungsleistungen politischer Akteure an die Medienlogiken zu internalisierten Handlungslogiken und politische Akteure handeln damit primär nach Medienlogiken und nicht mehr nach Politiklogiken. Diese vier Phasen nach Strömbäck (ebd.) sind, im Grunde, ein vereinfachtes differenzierungstheoretisches Modell, wie es z.B. Imhof (2006a) mit dem neuen Strukturwandel der öffentlichkeit beschreibt. Wenn aber Medialisierung als ein solcher quasi-historischer Wandel hin zu einer von Medienlogiken geprägten Gesellschaft im Allgemeinen, einer von Medienlogiken geprägten Politik im Besonderen gedacht wird, muss auch mitreflektiert werden, ob sich die Gesellschaft unabhängig der Medien wandelt. Wenn nämlich die Gesellschaft als statisch konzipiert wird und nur den Medien Wandel unterstellt wird, dann ist Medialisierung ein rein mediengetriebener Prozess. Wenn aber die Gesellschaft (bzw. die gesellschaftlichen Teilsysteme) jenseits der Medien in der quasi-historischen Phase nach dem zweiten Weltkrieg nicht statisch ist, dann kann Medialisierung als ein Aspekt oder Folge eines allgemeinen sozialen Wandels aufgefasst werden. Eben dieser Fragestellung widmen sich Blumler und Kavanagh (1999). Die Autoren gehen der Frage nach, was zu dem tiefgreifenden Wandel der politischen Kommunikation, welchen sie zwischen dem zweiten Weltkrieg und den 1990er Jahren beobachten, führt. Die Autoren machen eine Reihe gesellschaftlicher Veränderungen aus, welche zum Wandel politischer Kommunikation führen: Modernisierung, Individualisierung, Säkularisierung, Ökonomisierung, Ästhetisierung, Rationalisierung und Medialisierung. Das Erste, was bei diesen unterschiedlichen Strömen gesellschaftlicher Entwicklung auffällt, ist, dass die Autoren Medialisierung als eine Entwicklung auffassen, welche getrennt von dem Wandel politischer Kommunikation stattfindet und ein exogener Einflussfaktor auf diesen Wandel ist. Die Autoren führen aber nicht weiter aus, 
was genau Medialisierung ausmacht und, wie politische Kommunikation getrennt von Medialisierung zu denken ist. Als Medialisierung beschreiben die Autoren denn auch nur eine über Medien hergestellte Öffentlichkeit («media-constructed public sphere»), was die Frage aufwirft, ob es während und vor dem zweiten Weltkrieg keine massenmediale Öffentlichkeit gegeben haben soll. Auch die anderen Formen gesellschaftlichen Wandels beschreiben die Autoren nicht systematisch genug. Modernisierung, Individualisierung und Säkularisierung beispielsweise beschreiben alle den Prozess des intergenerationalen Wertewandels (Inglehart und Welzel 2005, 2009, 2010).

Der Beitrag von Blumler und Kavanagh (1999) ist aber dennoch darum wertvoll, weil er verdeutlicht, dass Medialisierung als längerfristiger Prozess nicht isoliert von sonstigem sozialen Wandel stattfindet. Die bestehenden Skizzen von Medialisierung als Prozess umreissen aber trotzdem alle in etwa dasselbe: In der Zeit nach dem zweiten Weltkrieg bis in etwa in die 1990er Jahre hinein findet Medialisierung statt, und ab dann haben wir es nur noch mit statischer Medialisiertheit zu tun. Ein solches Verständnis «abgeschlossener» Medialisierung ist aber in mindestens zweifacher Hinsicht problematisch.

Erstens gilt es erneut, individuelle Akteure handlungstheoretisch eben als Akteure aufzufassen. Das bedeutet nicht zuletzt, medialisiertes Handeln als sinngeleitet zu denken - auch wenn wir davon ausgehen, dass alle Akteure medialisiert handeln, stellt sich dennoch die Frage, warum sie medialisiert handeln. Erneut ist hier ein Verweis auf Abbildung 4 angebracht: Wenn für das Beispiel des Parlmamentes individuelle Parlamentarierinnen und Parlamentarier so handeln, dass die Präferenzen der Medien zum Ausdruck kommen, bedeutet das nicht automatisch, dass das zuungunsten der Präferenzen der Bürgerinnen und Bürger geschieht - Parlamentarierinnen und Parlamentarier können grundsätzlich ganz strategisch medialisiert handeln, um die Präferenzen der Bürgerinnen und Bürger zu erfüllen. Weiter sind alle politischen Akteure auf der Mikro-Ebene letztlich auch einem biologischen Zyklus unterworfen. Keine Politikerin, kein Politiker kommt medialisiert auf die Welt. Medialisierung als Anpassungsleistungen müssen entsprechend für jeden individuellen Akteur neu stattfinden, heute ebenso wie in der Vergangenheit und wie in der Zukunft. Die grossen und groben Veränderungen auf der Makro-Ebene, welche die Medialisierungsliteratur wie oben beschrieben skizziert, können als Kontextfaktoren begriffen werden: Die Bedingungen, unter denen sich individuelle politische Akteure medialisieren, haben sich im Laufe der Zeit verändert.

Zweitens ist die Fixierung auf die Periode zwischen dem Ende des zweiten Weltkrieges und in etwa dem Ende des 20. Jahrhunderts willkürlich. Diese Periode ist von grossen Innovationen und Umbrüchen geprägt, allen voran der breiten Einführung elektronischen Fernsehens in west- 
lichen Staaten. Andere Mediengattungen existierten aber auch während und vor des zweiten Weltkrieges, allen voran Zeitungen und Radio. Wenn also Medialisierung als Anpassungsleistungen an die Logiken von Massenmedien verstanden wird, dass müsste eine historisch sinnvolle Rekonstruktion von Medialisierung bei der Geburt von Massenmedien an und für sich beginnen. Eine derart umfassende Analyse von Medialisierung ist natürlich schwer umzusetzen, ein Stück weit auch, weil das «einfache» quasi-historische Narrativ (nach dem zweiten Weltkrieg ist die Gesellschaft in einem linearen Anstieg immer stärker medialisiert) gesprengt würde. Es ist aber hilfreich, die Vorstellung, dass Medialisierung so alt ist wie massenmedial hergestellte Öffentlichkeit, als gedankliche Stütze zu behalten. Diese Stütze rückt nämlich die dominierende eher pessimistische Sichtweise, dass Medialisierung immer gefährlichere Früchte trägt, in ein anderes Licht. Medialisierung bedeutet, dass Akteure Anpassungsleistungen an Medienlogiken erbringen - aber diese Medienlogiken sind nicht zwingend eine historisch fixe konstante, sondern selber auch von Wandel geprägt. Das bedeutet, dass auch gegenwärtig Wandel stattfindet, welcher Medialisierung bewirken kann. Um ein plakatives Beispiel zu nennen: Mit dem Smartphone aufgenommene fotografische Auto-Portraits, sogenannte «Selfies», sind in einem breiteren Kontext ein Medialisierungseffekt, denn Akteure passen ihr Handeln an bestimmte neue Medienlogiken an. Diese spezifische Anpassungsleistung findet auch auf solche Arten statt, dass tradierte normative Konventionen geritzt bis ignoriert werden, z.B. «Selfies» in Form von Selfies an Beerdigungen. Dieses medialisierte Ritual hat im Jahre 2013 sogar der amtierende US-amerikanische Präsident Barak Obama durchgeführt hat (Gibbs u. a. 2015).

Medialisierung kann also, zusammenfassend, als ein Prozess verstanden werden. Dieser Prozess fand allerdings nicht, wie bisweilen argumentiert, ausschliesslich in der Phase zwischen dem Ende des zweiten Weltkrieges und dem Ende des 20. Jahrhunderts statt. Medialisierung ist ein generalisierter Prozess, welcher auf der Ebene individueller Akteure beständig stattfindet (bzw. stattfinden kann) und durch andere soziale Entwicklungen mitbedingt ist.

\subsubsection{Medialisierung und parlamentarisches Handeln}

Aus meinen bisherigen Überlegungen zu Medialisierung dürfte sich abzeichnen, wie und warum ich Medialisierung als relevant für parlamentarisches Handeln erachte. Parlamentarierinnen und Parlamentarier, so meine Argumentation in Unterunterabschnitt 2.2.2, können in dem für diese Arbeit relevanten Kontext im Sinne der Präferenzen der Wählerschaft und im Sinne der Präferenzen der Medien handeln. Wenn Parlamentarierinnen und Parlamentarier im Sinne der Präferenzen von Medien handeln, dann erachte ich dieses Handeln als medialisiert. Medialisier- 
tes Handeln seitens der Parlamentarierinnen und Parlamentarier muss aber nicht zwangsläufig bedeuten, dass dieses Handeln zuungungsten der Präferenzen der Wählerschaft stattfindet, wie die Schnittmengen in Abbildung 4 visualisieren. Parlamentarierinnen und Parlamentarier können medialisiert handeln und mit diesem medialisierten Handeln den Präferenzen der Bürgerinnen und Bürger zur Entfaltung verhelfen.

Das bedeutet, dass ich Medialisierung als Treiber der Präferenzgenese erachte, welcher erklärt, warum Agenda Setting von Medien auf das Parlament stattfinden kann. Wie aber ist die zweite Seite der Medaille, das Handeln von Journalistinnen und Journalisten, im Kontext von Medialisierung zu verstehen? Dieser Aspekt spielt in der Medialisierungsliteratur eine untergeordnete Rolle. Medialisierung interessiert sich für Anpassungsleistungen vor allem politischer Akteure; allfällige Veränderungen in der journalistischen Arbeit werden, verständlicherweise, nicht unter dem Label der Medialisierung geführt - schliesslich soll journalistisches Handeln als Agens der Medialisierung gedacht werden. Bei genauerer Betrachtung spricht aber nichts dagegen, auch journalistisches Handeln als mehr oder weniger medialisiert aufzufassen. In der gängigen Ansicht zum Prozesscharakter von Medialisierung wird, wie in Unterunterabschnitt 3.2.1 beschrieben, eine quasi-historische Entwicklung zwischen dem Ende des zweiten Weltkrieges und in etwa dem Ende des 20. Jahrhunderts nachgezeichnet. Diese Entwicklung ist u.a. durch einen Prozess der Ausdifferenzierung der Medien gekennzeichnet, und diese Ausdifferenzierung besteht in einer stärkeren Professionalisierung, Kommerzialisierung und Emanzipierung von politischen Eliten. Medialisierung als Anpassungsleistungen politischer Akteure an dieser Veränderungen sind das dominante Motiv der Medialisierungsliteratur, aber bereits dieser quasi-historische Prozess der Ausdifferenzierung der Medien impliziert auch Veränderungen im Handeln seitens der Journalistinnen und Journalisten. Strömbäck (2008) beschreibt diese Veränderung als eine Art Emanzipierung der Medien, die sich nicht mehr an den Politiklogiken orientierten, sondern neu an den eigenen Medienlogiken. Nun sind diese quasi-historischen Schilderungen eben nur quasi-historisch, aber sie legen verständlich dar, dass Medialisierung auch Medien, d.h. Journalistinnen und Journalisten selber betrifft. Im Kontext der Argumentation in Unterunterabschnitt 2.2.2 lässt sich die Idee, dass auch Journalistinnen und Journalisten mehr oder weniger medialisiert handeln können, begründen: Auch Medien, d.h. Journalistinnen und Journalisten, können im Kontext von Parlamentsberichterstattung entweder ausschliesslich nach den eigenen Präferenzen handeln (Medienlogiken), oder sie können auch in einem solchen Masse nach den Politiklogiken handeln (Präferenzen von Bürgerinnen und Bürgern), dass die Berichterstattung diesen dienlich ist. 


\subsection{Rational Choice-Institutionalismus}

Medialisierung als theoretischer Rahmen ist ein Erklärungsvorschlag für die Motivstruktur von Parlamentarierinnen und Parlamentariern bei der Frage, warum sie im Sinne der Präferenzen der Medien handeln. In theoretischer Hinsicht ist damit allerdings noch zu wenig Substanz gegeben, um parlamentarisches Handeln wirklich zu verstehen. Parlamentarisches Handeln findet nämlich nicht in einem kontextlosen Raum statt, denn es ist an institutionelle Regeln und Verfahren gebunden. Diese Überlegung ist an und für sich trivial, denn jedes soziale Handeln ist an teils latente, teils formalisierte Regeln, Normen und Konventionen gebunden. Im Kontext des Parlamentes haben die institutionellen Regeln aber nebst einer solchen generellen handlungsstrukturierenden Funktion auch ganz unmittelbare und explizite Konsequenzen. Wenn Präferenzen als Motivstrukturen erklären, warum Akteure im Parlament handeln, dann erklären Institutionen, wie die Akteure handeln, um diese Präferenzen zu verwirklichen. Um dieses Wie des parlamentarischen Handelns als Folge der institutionellen Konfiguration des Parlamentes zu verstehen, ist eine dieses Phänomen umfassende theoretische Perspektive notwendig. Diese ist mit dem Rational Choice-Institutionalismus gegeben.

\subsubsection{Die Theorie nutzenmaxierender Akteure: Rational Choice}

Der Begriff «Rational Choice-Institutionalismus» verrät, dass es sich bei dieser Theorie um einen Verbund zweier Theorieaspekte handelt: Der Komponente des «Rational Choice» sowie der Komponente des Insitutionalismus. Weil die Komponente des Rational Choice bei Rational Choice-Institutionalismus lexikalischen Vorrang hat ${ }^{24}$ und, weil die Komponente des Rational Choice wohl umstrittener ist als die Komponente des Institutionalismus, ist es notwendig, zunächst auf die Rational Choice-Theorie einzugehen.

Die Grundannahme von Rational Choice als Handlungstheorie ist ausgesprochen simpel, da sparsam (Shepsle 2010, S. 17):

A rational individual is one who combines his or her beliefs about the external environment and preferences about things in that environment in a consistent manner.

Individuen haben Präferenzen, wollen also bestimmte Ziele eher erreichen als andere, und sie handeln nach bestem Wissen so, dass sie auf die Erreichung dieser Ziele hinwirken. Obschon

\footnotetext{
$24 \quad$ Wenn Rational Choice-Institutionalismus gültig sein soll, dann muss zunächst Rational Choice gültig sein.
} Mengenlogisch ausgedrückt: Rational Choice ist eine Teilmenge von Rational Choice-Institutionalismus. 
dieses Modell individuellen Handelns sehr einfach ist, ist es nach wie vor umstritten. So bemerken etwa Jarren und Donges (2011, S. 46):

Einwände gegen ökonomische Erklärungsansätze beziehen sich zumeist auf die Grundannahme, dass Akteure tatsächlich rational handeln. Sowohl aus der ökonomischen Theorie selbst heraus als auch aufgrund empirischer (und lebensweltlicher) Erfahrungen kann vielmehr davon ausgegangen werden, dass die Rationalität von Akteuren zumeist begrenzt ist.

Wenn Akteure in Tat und Wahrheit nicht rational im Sinne von zweckorientiert handeln, Rational Choice aber davon ausgeht, dass dem so ist, dann sieht sich die Rational Choice-Theorie mit einer unüberwindbaren Hürde konfrontiert. Wie ist dieses scheinbar fundamentale Problem zu lösen? Rund um Rational Choice gibt es viel Verwirrung, welche entsteht, weil es nicht eine einzige Rational Choice-Theorie gibt, sondern eher unterschiedliche Forschungslinien mit Rational Choice-Impetus (Quackenbush 2004). Die fundamentale Kritik an Rational Choice richtet sich denn auch nicht an gemässigtere Lesarten von Rational Choice, sondern vor allem auf eine eher «puristische» Auslegung (Goode 1997). Dieser Purismus besteht in der Annahme, wie sie etwa H. A. Simon (1955) schon früh kritisiert, dass Akteure zwar nicht allwissende Wesen sind, aber in ihrem Handeln dennoch insofern immer rational vorgehen sollen, als sie auch ihren eigenen Mangel an Informationen rational in Entscheidungen einberechnen. Diese Annahme widerspricht der biologischen Realität des Menschen, wie ich sie in Unterunterabschnitt 2.1.3 skizziere, denn das menschliche Denken ist nachweisbar nicht stets perfekt kalkuliert. So demonstriert nicht zuletzt verhaltensökonomische Forschung, dass zwischen einem idealisierten Modell von Rational Choice und den tatsächlichen kognitiven Vorgängen und den auf diesen Vorgängen basierenden Entscheidungen bisweilen grosse Unterschiede vorhanden sind; nicht zuletzt, weil das menschliche Denken anfällig für systematische Verzerrungen, sogenannte «kognitive Biases», ist (Kahneman 1994; Smith 1991; Tversky und Kahneman 1986). Dieses verzerrte Denken ist die Folge des in Unterunterabschnitt 2.1.3 beschriebenen schnellen Denkmodus des menschlichen Hirns, welcher über Routine und Heuristiken operiert. In einem nicht-puristischen Verständnis von Rational Choice, wie ich es für die vorliegende Arbeit verwende, ist die Idee der perfekten Rationalität nicht vorausgesetzt. Der Umstand, dass das idealisierte, puristische Verständnis von Rational Choice so gut wie definitiv immer empirisch falsch ist, hat dazu geführt, dass in der Literatur bisweilen der Begriff der «Bounded Rationality» (Camerer 1998; Jones 1999; Kahneman 2003) verwendet wird, um zu betonen, dass mit Rational Choice explizit die nicht-puristische Auslegung gemeint ist. Hechter und 
Kanazawa (1997) unterscheiden in Anlehnung an Ferejohn (1991) das puristische von dem realistischen Verständnis von Rational Choice mit den Begriffen «thick» und «thin», wobei das «dünne» Verständnis von Rational Choice der realistischeren Lesart entspricht (Hechter und Kanazawa 1997, S. 194):

Thin rational choice models are unconcerned with the particular values (or goals) which individuals pursue. These models are based on a small number of strong assumptions: for example, that whatever an individual's values may be, they must be stable and transitive (if someone prefers a to b, and b to c, they must prefer a to c).

In einem «dünnen» Verständnis bedeutet Rational Choice dementsprechend, dass Akteure Präferenzen haben können, und, dass sie generell danach streben, Präferenzen zu verwirklichen. Auch andere Kritikpunkte an Rational Choice betreffen in erster Linie die puristische Auslegung, so etwa die Annahme, dass Rational Choice bedeute, rationales Handeln sei nur egoistisches Handeln. Diese Kritik ist insofern ein Missverständnis, als damit die ökonomische Herkunft von Rational Choice als Verallgemeinerung von Rational Choice missverstanden wird. In einer ökonomischen Anwendung des Rational Choice-Modells ist der Nutzen, welchen ein Akteur als Marktteilnehmer zu maximieren sucht, der finanzielle Gewinn. Das bedeutet nicht, dass das Ziel der Gewinnmaximierung aus einem ökonomischen Modell das Ziel in allen anderen Anwendungen von Rational Choice darstellt. Es gibt keinen Grund, warum das Handeln von Akteuren für andere gesellschaftliche Kontexte nicht durch ganz andere Präferenzordnungen, z.B. solche mit utilitaristischen, nicht egoistischen Zielen, geleitet sein kann (Sen 1977). In einem Rational Choice-Modell für Katastrophenhilfe, um ein fiktives Beispiel heranzuziehen, ist der Nutzen, welcher maximiert werden soll, Menschenleben zu retten. Das Ziel, Menschenleben zu retten, ist aber ebensowenig ein universales Rational Choice-Ziel wie das Ziel, Gewinn zu maximieren, denn Rational Choice beschreibt, warum Akteure handeln, aber ohne vorzuschreiben, was für Motivstrukturen die Akteure haben müssen.

Nicht alle Kritik an der Rational Choice-Theorie ist aber die Folge der Verwechslung der puristischen mit der realistischen Variante von Rational Choice. Für die politikwissenschaftliche Verwendung der Rational Choice-Theorie ist bis heute die Kritik von Green und Shapiro (1996) wirkmächtig. Diese umfassende Kritik befasst sich nicht mit den Grundannahmen von Rational Choice und den Unterschieden zwischen einem puristischen und einem moderaten Verständnis von Rational Choice. Die Autoren kritisieren viel mehr die Art und Weise, wie Rational Choice in der politikwissenschaftlichen Forschung rezipiert wurde: Vielen theoretischen Modellierungen 
standen nur wenige empirische Arbeiten gegenüber. Das Problem ende aber nicht einfach mit einem Ungleichgewicht zwischen theoretischer und empirischer Arbeit. Auch fände eine Art Immunisierung statt, indem sich viele Rational Choice-Arbeiten de facto einer empirischen Untersuchung verschlössen. Zwar bemerkt Hug (2014), dass sich seit der Kritik von Green und Shapiro (1996) in empirischer Hinsicht die Situation gebessert habe, muss die theoretische Natur von Rational Choice-Modellen nach wie vor reflektiert werden. Bestimmte argumentative Immunisierungsstrategien zu Rational Choice sind nämlich nach wie vor im Umlauf, wie zum Beispiel das Argument, Rational Choice sei keine Handlungstheorie (Lovett 2006), und darum könne man Rational Choice nicht aufgrund eines mangelnden handlungstheoretischen Erklärungsgehaltes kritisieren. Diese Ansicht teile ich mit der vorliegenden Arbeit nicht. Das Ziel dieser Arbeit ist es, das Handeln von Parlamentarierinnen und Parlamentarierin und Journalistinnen und Journalisten zu untersuchen, und die theoretische Rahmung ist nur Mittel zu diesem Zweck. Es ist wichtig, dies zu betonen, denn einige Literatur zu Rational Choice verfolgt, wie Green und Shapiro (1996) argumentieren, das Ziel, Rational Choice zu «bestätigen», wobei allfällige Empirie der Mittel zum Zweck ist.

\subsection{2 «Institutions matter»: Rational Choice-Institutionalismus}

Wenn Parlamentarierinnen und Parlamentarier sowie Journalistinnen und Journalisten als Akteure gedacht werden, welche Präferenzen haben und nach diesen Präferenzen handeln, dann ist die theoretische Rahmung dieser Akteure naheliegenderweise (oder auch zwangsläufig) ein Rational Choice-Modell. Ein maximal komplexitätsreduziertes Rational Choice-Modell ignoriert dabei situative soziale Kontexte und fokussiert nur auf die Präferenzen an und für sich sowie auf die Frage, wie diese Präferenzen zu kollektiven Entscheidungen aggregieren. Ein solch reduziertes Modell ist aber oftmals unbefriedigend, um das reale Handeln politischer Akteure zu verstehen. Der Grund hierfür ist, wie Shepsle (1989) argumentiert, dass politische Akteure in der Realität nie in einem kontextlosen Raum handeln, sondern stets an formale Regeln, Prozeduren und Normen, also an Institutionen, gebunden sind. Rational Choice-Modelle nähern sich erst dann der Realität an, wenn also nicht nur bedacht wird, warum Akteure handeln (Präferenzen), sondern darüber hinaus auch, wie die Akteure genau handeln können. Diese an sich simple Idee, dass Instiutionen auch für Rational Choice-Modelle relevant sind, ist die Grundlage des Rational Choice-Institutionalismus. Rational Choice-Institutionalismus gehört, gemeinsam mit u.a. historischem Institutionalismus und soziologischem Institutionalismus, zur Theoriefamilie des «Neo-Institutionalismus» (Hall und R. C. R. Taylor 1996). Das Neue an neo-institutionalistischen Theorien gegenüber klassischen Theorien zu Institutionen ist 
der genaue Ort des Forschungsinteresses. Klassische Forschung zu Institutionen interessiert sich für Institutionen an und für sich, während sich neo-institutionalistische Ansätze für das Handeln von Akteuren interessieren, welches durch Institutionen ermöglicht und begrenzt wird (Immergut 1998).

Rational Choice-Institutionalismus ist eine elegante und plausible Erweiterung des Rational Choice-Modells, um das Handeln politischer Akteure zu analysieren. Rational ChoiceInstitutionalismus bleibt aber eine komplexitätsreduzierte Modellierung und ist nur begrenzt erklärungstauglich. Ein wichtiger Aspekt, der die Grenzen des Rational Choice-Institutionalismus aufzeigt, ist das Verständnis von Institutionen innerhalb dieser theoretischen Perspektive. Institutionen werden als Arrangements angesehen, welche lediglich existieren, weil sie Transaktionskosten senken (Koelble 1995). Eine solche Erklärung erklärt in Tat und Wahrheit weder soziologisch noch historisch irgendetwas. Rational Choice-Institutionalismus kann darum, erstens, nur Handeln analysieren, für welches die institutionelle Konfiguration als gegebener und stabiler exogener Faktor betrachtet wird. Zweitens ist Rational Choice-Institutionalismus für sich genommen theoretisch derart reduziert, dass das Handeln der Akteure erst dann als sinnvoll erachtet werden kann, wenn weitere sinnstiftende theoretische Ansätze zur Ergänzung herangezogen werden; Rational Choice postuliert, dass Akteure Ziele haben und diese anstreben, aber Rational Choice alleine sagt nichts darüber aus, was für Ziele die Akteure warum haben.

\subsubsection{Rational Choice-Institutionalismus und parlamentarisches Handeln}

In Unterunterabschnitt 2.3.4 erkläre ich, dass der Fokus der vorliegenden Arbeit auf den regulären Sessionen der Bundesversammlung liegt. Sessionen unterscheiden sich deutlich von der parlamentarischen Arbeit zwischen Sessionen. Sessionen stellen gewissermasen den öffentlich besser sichtbaren Teil der Parlamentsarbeit dar, in welchem das, was zwischen den Sessionen von den unterschiedlichen Kommissionen bearbeitet wurde, im Plenum behandelt wird. Die Sessionen bestehen aus unterschiedlichen Teilarenen, welche institutionell bedingt sind. Die erste und offensichtlichste institutionelle Komponente ist die die Unterscheidung nach zwei gleichberechtigte Kammern, dem Ständerat und dem Nationalrat . In den Plenarphasen der Sessionen behandeln sowohl der Nationalrat wie auch der Ständerat Geschäfte, welche spätestens zwei Wochen vor Beginn der Session im Sessionsprogramm traktandiert sind. Das Sessionsprogramm wird vom Büro des Nationalrates und vom Büro des Ständerates erstellt, unterstützt durch die Parlamentsdienste. Zu einem Teil der Geschäfte finden in beiden Kammern Abstimmungen statt (Eintretensdebatte, Detailberatung, Gesamtabstimmung, 
Schlussabstimmung) (Bundesgesetz, Verordnung oder Bundesbeschluss). In beiden Kammern wird während der Session also über Geschäfte gesprochen, und über einen Teil der Geschäfte wird abgestimmt. Das behandeln traktandierter Geschäfte stellt aber nur eine der Teilarenen während der Session dar. Eine weitere Teilarena sind Vorstösse: Während der Session können Mitglieder beider Kammern unterschiedliche Arten von Vorstössen (einfache Anfragen, Interpellationen, Postulate, Motionen sowie parlamentarische Initiativen) einreichen. Vorstösse werden meistens von individuellen Parlamentarierinnen und Parlamentariern eingereicht, seltener auch von Fraktionen. Eine dritte Teilarena schliesslich bilden die Fragestunden im Nationalrat. In jeder regulären Session finden zwei Fragestunden statt, jeweils zu Beginn der zweiten und der dritten Sessionswoche, bei denen Mitglieder des Bundesrates Fragen von Nationalrätinnen und Nationalräten beantworten. Die Fragen werden aber nicht spontan vor Ort gestellt, sondern schriftlich bis am Mittwoch der jeweiligen Vorwoche eingereicht.

Die institutionelle Konfiguration der Bundesversammlung während der Session besteht also aus drei Teilarenen: Erstens, regulären, traktandierten Geschäften im National- und im Ständerat; zweitens, den eingereichten Vorstössen von National- und Ständerat; und drittens, den Fragestunden im Nationalrat. Aus der Perspektive des Rational Choice-Institutionalismus bedeutet das, dass es sich bei diesen drei Teilarenen um drei unterschiedliche institutionelle Konfigurationen handeln, in denen die relevanten Akteure, also die Parlamentarierinnen und Parlamentarier, auf unterschiedliche Art gemäss ihren Präferenzen handeln. Die Möglichkeiten des Handelns sind dabei nicht für jede der Teilarenen identisch. Die Inhalte der regulären Geschäfte können während der Session nicht verändert werden. Darum ist bei regulären Geschäften während der Session der Ort der Entscheidungsfindung das Abstimmen über Geschäfte. Anders sieht es bei Vorstössen aus. Jedes individuelle Mitglied sowohl des National- wie auch des Ständerates kann sowohl Vorstösse während der Session einreichen und diese inhaltlich frei gestalten. Allerdings werden Vorstösse frühestens in der darauffolgenden Session behandelt; es findet also keine Entscheidungsfindung zu den eingereichten Vorstössen in der jeweils laufenden Session statt. Die Fragestunden schliesslich sind in Betreff der Handlungsmöglichkeiten identisch mit den Vorstössen, aber den Mitgliedern nur einer Kammer vorbehalten: Nationalrätinnen und Nationalräte können Fragen einreichen und sie inhaltlich frei gestalten. Fragen werden direkt in den Fragestunden (bzw. bei vielen Fragen zusätzlich schriftlich) von Mitgliedern des Bundesrates beantwortet.

Die institutionelle Konfiguration spielt auch für Journalistinnen und Journalisten eine Rolle. Medien können grundsätzlich das Geschehen während Sessionen von Aussen mitverfolgen und für ihre Berichterstattung Parlamentsprotokolle verwenden. Viele Medien aber sind mit 
Journalistinnen und Journalisten vor Ort in Bern, der Schweizer Bundesstadt, wo auch das Bundeshaus steht und die Sessionen tagen. Zahlreiche Journalistinnen und Journalisten sind dabei nicht nur in Bern vor Ort, sondern haben als akkreditierte Bundeshauskorrespondentinnen und -korrespondenten Zugang zum Bundeshaus. Der Zugang ist dabei nicht ein totaler: Akkreditierte Journalistinnen und Journalisten dürfen nicht die zwei Ratssäle, Sitzungszimmer und die Zuschauertribünen betreten. Der Rest des Bundeshauses, inklusive Vorzimmer der beiden Ratssäle sowie der Wandelhalle des Nationalrates, steht ihnen aber offen.

\subsection{Theoriesynthese und Hypothesen}

Die vorherigen Abschnitte sollten jene theoretischen Perspektiven einführen, welche dazu dienen, den Gegenstand und die Fragestellungen dieser Arbeit greifbar zu machen. Zunächst habe ich Agenda Setting als Theorie vorgestellt, welche sich dafür interessiert, wie unterschiedliche thematische Agenden einander beeinflussen. Agenda Setting ist für die vorliegende Arbeit darum nützlich, weil das Handeln nach der Präferenzfamilie der Politiklogiken oder nach der Präferenzfamilie der Medienlogiken gut in das Konzept von Agenda Setting überführt werden kann. Um diese Überführung aber plausibel zu machen, muss ein Agenda Setting-Modell noch um weitere theoretische Impulse angereichert werden. Blosses Agenda Setting impliziert, dass eine Agenda die andere kraft ihrer Salienz beeinflusst - was in der einen Agenda prominent ist, beeinflusst eher die andere Agenda. Eine solche Auffassung von Parlament und Medien würde an und für sich bereits genügen, um eine empirische Forschungsarbeit durchzuführen. Es ist ohne grössere Probleme möglich, zu vergleichen, was für Inhalte im Parlament vorkommen, was für Inhalte in der massenmedialen Berichterstattung, um anschliessend allfällige Ähnlichkeiten in der Prominenz von Themen als Agenda Setting-Effekt zu deklarieren. Die konzeptuell-normative Basis der vorliegenden Arbeit ist aber, wie in Unterunterabschnitt 2.2.2 dargelegt, eine fundamental handlungstheoretische Auffassung von Parlament und Medien. Das bedeutet, dass ein blosser Vergleich von Agenden mit anschliessender Proklamation von Agenda Setting-Effekten (oder deren Abwesenheit) nicht genügt. Ein solches Vorgehen genügt darum nicht, weil Parlament und Medien handlungstheoretisch aus individuellen Akteuren bestehen, und allfällige Agenda Setting-Effekte sind die Folge des Handelns dieser Akteure. Eine handlungstheoretische Perspektive erschöpft sich aber nicht in der trivialen Feststellung, dass die Akteure auf Mesoebene, Parlament und Medien, aus Akteuren auf den Mikro-Ebene, Parlamentarierinnen und Parlamentariern sowie Journalistinnen und Journalisten, bestehen. Handlungstheoretisch interessieren neben der Frage des Was (Agenda Setting) auch die Fragen des Warum und des Wie. Die Frage des Warum soll Aufschluss über die Motivstrukturen der 
involvierten Akteure im Agenda Setting-Prozess geben. Die Frage des Wie soll plausible Erwartungshaltungen hinsichtlich der konkreten Abläufe von Agenda Setting generieren und prüfbar machen.

Antworten auf die Frage des Warum suche ich in Form der Medialisierungstheorie. Medialisierung bedeutet, wie in Unterabschnitt 3.2 argumentiert, Anpassungsleistungen an die Logiken der Medien. Im Kontext der konzeptuellen Basis der vorliegenden Arbeit, der prozeduralisierten Volkssouveränität als Prinzipal-Agent-Beziehung zwischen Bürgerinnen und Bürgern und Parlamentarierinnen und Parlamentariern, bedeutet Medialisierung das Handeln im Sinne der Präferenzfamilie der Medienlogiken. Medialisiertes Handeln im parlamentarischen Kontext betrifft sowohl Parlamentarierinnen und Parlamentarier als auch Journalistinnen und Journalisten. Beide Akteursgruppen können nämlich stärker im Sinne der Politiklogiken oder stärker im Sinne der Medienlogiken handeln. Die Präferenzfamilien der Politiklogiken und der Medienlogiken sind dabei, wie in Abbildung 4 visualisiert, nicht zwingend exklusiv: Medialisiertes Handeln kann Handeln sein, welches dennoch zugunsten der Politiklogiken ist.

Wie genau können aber Parlamentarierinnen und Parlamentarier sowie Journalistinnen und Journalisten handeln? Dieses Wie wird durch die theoretische Komponente des Rational Choice-Institutionalismus ergänzt. Während der Session können Parlamentarierinnen und Parlamenterier in unterschiedlichen parlamentarischen Teilarenen aktiv sein. Eine erste Handlungsoption ist das Abstimmen bei den regulär traktandierten Geschäften, sowohl im Nationalwie auch im Ständerat. Eine zweite Handlungsoption, welche ebenfalls für Mitglieder beider Kammern offen ist, ist das Einreichen von Vorstössen. Eine dritte Handlungsoption, welche Mitgliedern des Nationalrates vorbehalten ist, ist das Einreichen von Fragen für die Fragestunden. Journalistinnen und Journalisten haben nur eine Handlungsoption: Die Auswahl von Themen aus den drei parlamentarischen Teilarenen für die Berichterstattung.

Die erste Forschungsfrage fragt danach, in welchem Masse Parlamenterierinnen und Parlamentarier nach Politiklogiken und in welchem Masse nach Medienlogiken handeln:

- FF1: In welchem Masse handeln Parlamentarierinnen und Parlamentarier nach Politiklogiken und in welchem Masse nach Medienlogiken?

Die aus der Rational Choice-Perspektive beschriebenen Handlungsmöglichkeiten bedeuten, dass diese Frage für die unterschiedlichen parlamentarischen Arenen - traktandierte Geschäfte, Vorstösse und Fragestunden - separat untersucht werden müssen. Agenda Setting beudeutet im Kontext dieser Forschungsfrage, dass das Handeln der Parlamentarierinnen und Parlamentarier in den unterschiedlichen Teilarenen durch Medieninhalte beeinflusst werden kann, da 
die betroffenen Akteure medialisiert sein können, also im Sinne der Medienlogiken handeln können. An dieser Stelle drängt sich die Frage auf, inwiefern es eine Präferenz der Medien, d.h. der Journalistinnen und Journalisten ist, dass Parlamentarierinnen und Parlamentarier Inhalte der Berichterstattung in Ihr parlamentarisches Handeln einfliessen lassen. Ob ein solcher Einfluss eine direkte Präferenz darstellt, ist nicht ohne Weiteres, also nicht a priori klar. Es mag sein, dass Journalistinnen und Journalisten, oder ganzen Medien auf Ebene der Organisationsstrategie, daran gelegen ist, dass die Berichterstattung eine Wirkung auf Parlamentarierinnen und Parlamentarier als politische Eliten hat. Ein Einfluss von Medieninhalten auf das Handeln von Parlamentarierinnen und Parlamentariern stellt a priori, also generalisiert, aber eine indirekte Präferenz der Medien dar. Dadurch, dass bestimmte Themen Gegenstand medialer Berichterstattung sind, gehören sie zu den thematischen Präferenzen der betroffenen Medien. Wenn Parlamentarierinnen und Parlamenterier diese Inhalte in ihr parlamentarisches Handeln einfliessen lassen, dann reproduzieren sie damit die Präferenzen von Journalistinnen und Journalisten.

Die ersten zwei Hypothesen formuliere ich mit Bezug auf das Abstimmen im National- und im Ständerat:

- Hypothese H1: Abstimmungen im Nationalrat sind nicht durch die vorangehende Berichterstattung zu den betroffenen Geschäften beeinflusst.

- Hypothese H2: Abstimmungen im Ständerat sind nicht durch die vorangehende Berichterstattung zu den betroffenen Geschäften beeinflusst.

Die in den Hypothesen formulierte Erwartung ist, dass das Abstimmungsverhalten ${ }^{25}$ weder im Nationalrat noch im Ständerat durch Berichterstattung in den Medien beeinflusst wird. Abstimmungen zu Geschäften sind individuell wie auch parteipolitisch strategische und im Voraus reflektierte Entscheidungen. Mit diesen Hypothesen prüfe ich, ob die Vermutung, dass das im Voraus bedachte Abstimmen Vorrang vor aktueller Berichterstattung geniesst, zutrifft. Die Situation bei Vorstössen und den nationalrätlichen Fragestunden schätze ich anders ein:

- Hypothese H3: Die eingereichten Vorstösse sind durch die vorangehende Berichterstattung beeinflusst.

- Hypothese H4: Die Fragen in den Fragestunden des Nationalrates sind durch die vorangehende Berichterstattung beeinflusst.

$25 \quad$ Den Begriff Abtsimmungsverhalten verwende ich nur der Geläufigkeit halber. Das Abstimmen als Abstimmungsverhalten zu beschreiben, ist unglücklich, denn es handelt sich um Abstimmungshandeln. 
Ich gehe davon aus, dass sowohl die Vorstösse wie auch die Fragestunden im Nationalrat durch aktuelle Medienberichterstattung innerhalb der Session beeinflusst werden. Weiter gehe ich davon aus, dass die Vorstösse und die Fragestunden in unterschiedlichem Masse durch aktuelle Medienberichterstattung beeinflusst werden, wie ich mit der folgenden Hypothese postuliere:

- Hypothese H5: Fragen in den Fragestunden sind stärker durch Berichterstattung beeinflusst als die eingereichten Vorstösse.

Die Fragestunden werden im Gegensatz zu eingereichten Vorstössen bereits innerhalb der jeweiligen Session behandelt. Das bedeutet, das Fragestunden ein ideales Fenster für Nationalrätinnen und Nationalräte darstellen, um aktuelle Themen aus den Medien aufzugreifen und den Bundesrat mit diesen zu konfrontieren. Fragestunden sind also von ihrer institutionellen Natur her dafür vorgesehen, aktuelle Themen kurzfristig auf eine Teilarena der parlamentarischen Agenda zu bringen.

Wenn ich auch, gesamthaft gesehen, davon ausgehe, dass Parlamentarierinnen und Parlamentarier ein Stück weit aktuelle Inhalte von Medien in ihr Handeln einbringen, gehe ich davon aus, dass sie insgesamt stärker an den Politiklogiken denn an den Medienlogiken orientiert sind:

- Hypothese H6: Parlamentarierinnen und Parlamentarier handeln stärker nach Politiklogiken als nach Medienlogiken.

Die Frage, wie stark Parlamentarierinnen und Parlamentarier insgesamt an Politiklogiken und wie stark an Medienlogiken orientiert sind, ist getrennt von der weiterführenden Frage, ob jener Teil ihres Handelns, welcher medialisiert ist und damit die Präferenzen der Medien reproduziert, darüber hinaus das Ziel hat, zugunsten der Politiklogiken zu sein, also zugunsten der Präferenzen der Bürgerinnen und Bürger. Das ist eine Konsequenz des mengentheoretischen Verhältnisses der Präferenzfamilien der Politiklogiken und der Medienlogiken, welches in Abbildung 4 visualisiert ist. Beispielsweise ist eine Konfiguration denkbar, in welcher der Grossteil des Handelns ausschliesslich nach den Politiklogiken erfolgt und ein kleiner Teil nach Medienlogiken. Dieser kleine Teil kann nun ausschliesslich nach Medienlogiken erfolgen, oder, er kann nach den Medienlogiken und gleichzeitig nach den Politiklogiken erfolgen, womit er letztlich wieder ein Teil der Politiklogiken wird. Anders formuliert wäre in diesem fiktiven Beispiel ein kleiner Teil des Handelns medialisiert und zugunsten der Politiklogiken. Wie gross oder wie klein der Anteil des medialisierten Handelns ist, ist Gegenstand von Hypothese H6. Ob dieser medialisierte Anteil eher zugunsten der Politiklogiken oder zugunsten der Medienlogiken ist, ist Gegenstand einer separaten Hypothese: 
- Hypothese H7: Parlamentarierinnen und Parlamentarier handeln medialisiert als Mittel zum Zweck zugunsten der Politiklogiken.

Die Hypothesen H6 und H7 stehen in keinem kausalen Verhältnis zueinander, denn Hypothese H7 fragt nach dem Zweck des medialisierten Handelns, unabhängig davon, wie umfangreich dieses Handeln ist. Hypothese H6 hingegen interessiert sich nicht für die Schnittmenge der Medienlogiken und der Politiklogiken, sondern nur für die sich ausschliessenden Mengen der exklusiven Politiklogiken auf der einen Seite, der exklusiven Medienlogiken sowie der Schnittmenge zwischen Medienlogiken und Politiklogiken auf der anderen Seite.

Die zweite Forschungsfrage widmet sich den Präferenzen von Journalistinnen und Journalisten im Kontext ihrer Berichterstattung über das Parlament während der Session:

- FF2: In welchem Masse handeln fournalistinnen und fournalisten in der Berichterstattung über das Parlament nach Medienlogiken und in welchem Masse nach Politiklogiken?

Wie in Unterunterabschnitt 2.2.3 argumentiert, gibt es gute Gründe, davon auszugehen, dass Medien gesamthaft über das Parlamentsgeschehen während der Session berichten, und dabei alle drei Teilarenen abdecken. Ich gehe aber davon aus, dass nicht alle Teilarenen im gleichen Umfang behandelt werden:

- Hypothese H8: Medien berichten am meisten über die traktandierten Geschäfte, weniger über die eingereichten Vorstösse und am wenigsten über die Fragestunden im Nationalrat.

Am meisten Berichterstattung erwarte ich über die regulären, traktandierten Geschäfte, und zwar aus mehreren Gründen. Erstens ist dieser Teil des Parlamentsgeschehens, verglichen mit der Anzahl eingereichter Vorstösse und dem Umfang der Fragestunden, der grösste. Zweitens sind die regulären Geschäfte der Ort, an dem unmittelbare Entscheidungspolitik stattfindet; Fragen in der Fragestunde sind ohne Konsequenz (daraus entstehen keine Erlasse), und Vorstösse werden erst in Zukunft politisch aktuell. Traktandierte Geschäfte stellen denn auch einen wesentlichen Teil der in Abbildung 4 visualisierten Schnittmenge zwischen den Medienlogiken und den Politiklogiken dar. Das bedeutet also, dass ich weiter davon ausgehe, dass nicht nur Parlamentarierinnen und Parlamentarier, sondern auch Journalistinnen und Journalisten sich nach Politiklogiken richten. Allerdings erwarte ich, dass sich Journalistinnen und Journalisten weniger stark nach Politiklogiken richten als Parlamentarierinnen und Parlamentarier:

- Hypothese H9: Journalistinnen und Journalisten handeln in gleichem Umfang nach Medienlogiken wie nach Politiklogiken. 
Die dritte Forschungsfrage bringt die zeitliche Perspektive in die Untersuchung, indem sie sich dem Wandel der in den ersten beiden Forschungsfragen angesprochenen Handlungsdynamiken widmet:

- FF3: Wandeln sich die Präferenzen von Parlamentarierinnen und Parlamentariern sowie von fournalistinnen und fournalisten im Zuge der Medienkrise?

$\mathrm{Zu}$ den ersten beiden Forschungsfragen lassen sich theoriegeleitete Hypothesen ableiten. Bei der dritten Forschungsfrage ist das nicht der Fall. Wenn auch insbesondere die Medialisierungstheorie einen inhärenten Prozesscharakter hat, wie ich in Unterunterabschnitt 3.2.1 beschreibe, ist dies eine zu wenig fundierte Grundlage, um gut begründete Hypothesen für Veränderungen, welche durch die Medienkrise verursacht sind, herzuleiten. Darum verzichte ich für die dritte Forschungsfrage auf Hypothesen und gehe stattdessen explorativ vor.

Open Access Dieses Buch wird unter der Creative Commons Namensnennung - Nicht kommerziell 4.0 International Lizenz (http://creativecommons.org/licenses/by-nc/4.0/deed. de) veröffentlicht, welche für nicht kommerzielle Zwecke die Nutzung, Vervielfältigung, Bearbeitung, Verbreitung und Wiedergabe in jeglichem Medium und Format erlaubt, sofern Sie den/die ursprünglichen Autor(en) und die Quelle ordnungsgemäß nennen, einen Link zur Creative Commons Lizenz beifügen und angeben, ob Änderungen vorgenommen wurden.

Etwaige Abbildungen oder sonstiges Drittmaterial unterliegen ebenfalls der genannten Creative Commons Lizenz, sofern sich aus der Abbildungslegende oder der Quellreferenz nichts anderes ergibt. Sofern solches Drittmaterial nicht unter der genannten Creative Commons Lizenz steht, ist eine Vervielfältigung, Bearbeitung oder öffentliche Wiedergabe nur mit vorheriger Zustimmung des betreffenden Rechteinhabers oder auf der Grundlage einschlägiger gesetzlicher Erlaubnisvorschriften zulässig. 


\section{Forschungsdesign und Methoden}

\subsection{Forschungsdesign}

Van Aelst und Walgrave (2011) bemerken, dass die Ergebnisse der Forschung zu Agenda Setting zwischen Parlament und Medien stark davon abhängen, was für Methoden, oder breiter, Forschungsdesigns, gewählt werden. Konkret vergleichen die Autoren die Diskrepanz der Ergebnisse zwischen quantitativen Analysen von Medieninhalten und parlamentarischen Inhalten sowie quantitativen oder qualitativen Befragungen von Parlamentarierinnen und Parlamentariern. Es gibt zwei grobe, generelle Herangehensweisen an diese Problematik. Die erste ist, die Schwierigkeiten mit dem gewählten Design und den dazugehörigen Methoden bewusst anzusprechen und die gewonnenen Ergebnisse entsprechend kritisch zu reflektieren. Die zweite Herangehensweise ist, beide Designs, das auf Inhaltsanalyse und das auf Befragungen fokussierte, in einem einzigen Forschungsprogramm zu vereinen. Für die vorliegende Arbeit habe ich mich für die zweite Herangehensweise entschlossen.

Eine Kombination unterschiedlicher Forschungsdesigns und Methoden zum Zwecke der besseren Erfassung eines Forschungsgegenstandes wird metaphorisch als Triangulation bezeichnet. Die Grundidee von Triangulation ist simpel. Denzin (1970, S. 13) beschreibt Triangulation folgendermassen:

If each method leads to different features of empirical reality, then no single method can ever capture all the relevant features of that reality; consequently, sociologists must learn to employ multiple methods in the analysis of the same empirical events.

Wenn auch die Grundidee von Triangulation simpel ist, herrscht Uneinigkeit darüber, wie genau Triangulation umzusetzen ist. Ein Teil der Uneinigkeit rührt daher, dass nicht immer klar ist, ob und wie sich Triangulation vom Konzept des «Mixed Methods Research» unterscheidet oder unterscheiden soll. Johnson und Onwuegbuzie (2004) bemerken in einem Übersichtsartikel, dass es keine einheitliche Definition von Mixed Methods Research gibt und sie schlagen darum eine solche vor (ebd., S. 132):

Mixed methods research is the type of research in which a researcher or team of researchers combines elements of qualitative and quantitative research approaches (e.g., use of qualitative and quantitative viewpoints, data collection, analysis, inference techniques) for the broad purposes of breadth and depth of understanding and corroboration. 
Eine solche breite Definition ist wohl prinzipiell kompatibel mit der Idee der Triangulation. So gibt es denn auch Autoren, welche Triangulation als die gleichzeitige Verwendung qualitativer und quantitiativer Methoden definieren (Jick 1979). Das ursprüngliche Verständnis von Triangulation zielt aber nicht auf das Kombinieren quantitativer und qualitativer Methoden ab, sondern auf das Kombinieren unterschiedlicher qualitativer Methoden (Denzin 2012). Mixed Methods Research hat den Ursprung bzw. die häufigste Verwendung im Gesundheitsbereich (Sale, Lohfeld und Brazil 2002). Ein typisches Design in einem solchen Kontext ist eine qualitative Vorstudie für eine quantitative Hauptstudie, oder allenfalls eine qualitative «Nachstudie» nach der quantitativen Hauptstudie. Mixed Methods Research hat schwerpunktmässig somit zwei Haupteigenschaften: Der qualitative und der quantitative Teil stehen in einem sequentiellen Verhältnis, und der Hauptfokus liegt auf dem quantitativen Teil. Flick u. a. (2012) erachten Mixed Methods Reasearch und Triangulation gemäss der angesichts der unterschiedlichen dahinterstehenden Konzepte als zwar prinzipiell separat, aber als letztlich doch vereinbar: Triangulation hat zum Ziel, denselben Gegenstand mit unterschiedlichen qualitativen Methoden zu untersuchen, während Mixed Methods Research quantitative und qualitative Methoden kombiniert, aber nicht denselben Gegenstand trianguliert. Es gibt a priori keinen Grund, warum diese zwei Prinzipien nicht kombiniert werden können, indem also mit qualitativen und quantitativen Methoden derselbe Gegenstand untersucht wird. Wenn ein Mixed Methods-Design als Triangulation umgesetzt wird, ändern sich diese Haupteigenschaften: Der qualitative und der quantitative Teil stehen in einem parallelen Verhältnis - derselbe Gegenstand wird untersucht -, und die qualitativen und quantitativen Daten werden als gleich bedeutsam in Betreff ihrer Aussagekraft betrachtet ${ }^{26}$. Das ist das Design der vorliegenden Arbeit.

Leech und Onwuegbuzie (2007) haben eine Typologie innerhalb der Mixed MethodsDenkschule erarbeitet, mit der das verwendete Design in dieser Arbeit genauer beschrieben werden kann. Die Autoren unterscheiden nach acht Mixed Methods-Designs, welche sich anhand von drei Dimensionen unterscheiden: Der «Mixing»-, der zeitlichen und der «Betonungs»Dimension. Für die «Mixing»-Dimension unterscheiden die Autoren nach teilweisen und vollen Mixed Methods. Teilweise Mixed Methods sind Designs, bei denen es einen qualitativen und einen quantitativen Teil gibt, wobei die Datenerhebung und die Datenauswertung jeweils zwei Mal qualitativ und zwei Mal quantitativ ist. Als volle Mixed Methods betrachten die

26 Howe (2012) beschreibt den Unterschied zwischen einem sequentiellen und einem parallelen Verhältnis als Unterschied zwischen einer «disjunctive», also trennenden, und einer «conjunctive», also verbindenden Anwendung von Triangulation. Eine solche Unterscheidung scheint mir weniger präzise als die Unterscheidung nach sequentiell und parallel; nicht zuletzt, weil die Metapher der Triangulation impliziert, dass Triangulation eben darum zustande kommt, weil die Methoden in einem parallelen Verhältnis stehen. Eine Triangulation, bei der die Methoden «disjunct», also getrennt sind, sorgt bloss für definitorische Verwirrung. 
Autoren Designs, bei denen es auch zwischen Datenerhebung und Datenauswertung einen Methodenwechsel gibt, z.B., indem qualitative erhobene Daten quantitativ ausgewertet werden. Die Zeitdimension betrifft die Frage, ob der qualitative und der quantitative Teil in einem sequentiellen oder in einem parallelen Verhältnis stehen - und die Zeitdimension ist letztlich entscheidend für die Klassifikation eines Designs als Triangulation; nur paralleles Vorgehen ist wirkliche Triangulation im engeren Sinn. Die Betonungs-Dimension schliesslich ist der bereits erwähnte Aspekt, dass es zu entscheiden gilt, welchem Teil des Designs, dem qualitativen oder dem quantitativen, in der Untersuchung mehr Aussagekraft und Bedeutung zugesprochen wird. Die Eigenschaften des Designs dieser Arbeit sind ein volles Mixing, ein paralleles Verhältnis der qualitativen und quantitativen Teile sowie eine gleichwertige Bedeutung der qualitativen und quantitativen Ergebnisse. Die detaillierten Aspekte des Designs sind in den nachfolgenden Abschnitten 4.2 bis 4.4 besprochen.

\subsection{Datenerhebung}

Die Datenerhebung besteht aus zwei separaten Teilen: Der Inhaltsanalyse von Medien und Parlamentsprotokollen zum einen, Leitfadeninterviews zum anderen. Das Vorgehen für die Inhaltsanalyse ist in Unterunterabschnitt 4.2.1 beschreiben, das Vorgehen für die Leitfadeninterviews in Unterunterabschnitt 4.2.2.

\subsubsection{Inhaltsanalyse}

Das Ziel der Inhaltsanalyse in der vorliegenden Arbeit ist es, zutage zu fördern, welche konkreten Themen wann und wie oft in welchen parlamentarischen Arenen sowie in den Medien vorkommen, sowie, zu welchen Politikfeldern die einzelnen konkreten Themen in den parlamentarischen Arenen und in den Medien zugehörig sind. Mit dieser knappen Beschreibung ist im Grunde erschöpfend aufgezählt, was für Variablen erhoben werden, nämlich:

- Konkretes Thema der Untersuchungseinheit

- Politikfeld der Untersuchungseinheit

- Erhebungseinheit (Wo)

- Datum der Erhebungseinheit (Wann)

Die Anzahl der inhaltsanalytisch erhobenen Variablen ist also sehr überschaubar, aber nicht ganz trivial. Ein zentrale Entscheidung für die Inhaltsanalyse betrifft nämlich die Frage, welche Inhalte des Parlamentes und welche Inhalte der Medienstichprobe genau untersucht werden 
sollen - was also die Erhebungs- und die Untersuchungseinheiten sein sollen. In Unterabschnitt 3.1 beschreibe ich, dass sich die Agenda Setting-Forschung in erster Linie der Frage der Themen widmet. Darum fokussiere ich auch bei der Inhaltsanalyse auf diese direkte inhaltliche Komponente und nicht auf erweiterte Aspekte, z.B. die Frage, wie genau die Themen präsentiert werden. Für die Inhaltsanalyse sind zwei Kernvariablen erfasst: Die konkreten Themen sowie die Politikfelder. Diese zwei Variablen, das jeweilige Thema und das jeweilige Politikfeld einer Untersuchungseinheit, sind ähnliche Variablen, aber nicht identische. Bevor ich aber erkläre, was die konkreten Themen und was die Politikfelder sind, ist ein Verweis auf Tabelle 2 angebracht. In Tabelle 2 ist zusammengefasst, was die Erhebungs- und was die Untersuchungseinheiten für die Kernvariablen Thema und Politikfeld für die unterschiedlichen Quellen sind.

Tabelle 2: Erhebungs- und Untersuchungseinheiten für die Kernvariablen Thema und Politikfeld.

\begin{tabular}{lll}
\hline & Erhebungseinheit & Untersuchungseinheit \\
\hline Zeitungen & Zeitungsausgabe & Zeitungsartikel \\
Fernsehen & Einzelne Sendung & Beitrag innerhalb der Sendung \\
Radio & Einzelne Sendung & Beitrag innerhalb der Sendung \\
Parlament: Geschäfte NR & Tagesdebatte & Wortmeldung \\
Parlament: Geschäfte SR & Tagesdebatte & Wortmeldung \\
Parlament: Vorstösse & Eingereichte Vorstösse pro Tag & Vorstoss \\
Parlament: Fragestunden NR & Fragestunde & Wortmeldung \\
Parlament: Abstimmungen NR & Geschäft & Geschäft \\
Parlament: Abstimmungen SR & Geschäft & Geschäft \\
\hline
\end{tabular}

Für die Medien sind die Erhebungseinheiten einzelne Zeitungsausgaben bzw. einzelne Sendungen. Die Untersuchungseinheiten für Medien sind einzelne Artikel in Zeitungen und einzelne Beiträge in Sendungen. Die Erhebungseinheit, welche auf Seiten der Medien von Interesse ist, ist also der Korpus der Berichterstattung des jeweiligen Mediums an einem Tag. Die Erhebungsund Untersuchungseinheiten auf Seiten das Parlamentes sind weitgehend in derselben Logik festgelegt: Bei den traktandierten Geschäften des National- und des Ständerates ist die Erhebungsheinheit die an diesem Tag geleistete Arbeit, also die Tagesdebatte, und die Untersuchungseinheiten sind einzelne Wortmeldungen. Für Vorstösse ist die Erhebungseinheit die Gesamtheit der an einem Tag eingereichten Vorstösse und die Untersuchungseinheit der einzelne Vorstoss. Bei Fragestunden sind, analog dazu, die gesamten Fragestunden die Erhebungseinheiten und die einzelnen Wortmeldungen die Untersuchungseinheiten ${ }^{27}$. Einzig bei den Abstimmungen im National- und im Ständerat sieht es anders aus: Hier sind sowohl die

\footnotetext{
27 Die meisten Fragen in den Fragestunden bestehen aus zwei Wortmeldungen: Der Frage einer Nationalrätin oder eines Nationalrates und der Antwort eines Bundesratsmitglieds.
} 
Erhebungs- wie auch die Untersuchungseinheiten einzelne Geschäfte. Für Abstimmungen würde es, logischerweise, keinen Sinn machen, diese nach einzelnen Wortmeldungen zu erfassen, denn Abstimmungen erfolgen auf der Ebene von Geschäften. Bei Abstimmungen wird also lediglich erfasst, wie viele Ja- und Nein-Stimmen es gibt. Die Ebene der Geschäfte ist praktischerweise auch für die anderen parlamentarischen Untersuchungseinheiten relevant: Alle Wortmeldungen zu einem Geschäft sowie alle Wortmeldungen zu einer Frage in der Fragestunde haben automatisch dasselbe Thema und dasselbe Politikfeld. Dieser Automatismus kommt darum zustande, weil unterschiedliche Wortmeldungen zu einem einzelnen Geschäft alle dieses eine Geschäft betreffen. Das klingt ein wenig verworren, meint aber ganz etwas Banales: Bei einem Geschäft im Parlament kann es mehrere Wortmeldungen geben.

Was genau ist unter konkreten Themen und Politikfeldern zu verstehen? Seit der Studie von McCombs und Shaw (1972) besteht das übliche Vorgehen in der Agenda Setting-Forschung darin, die zu vergleichenden Agenden deduktiv als «Issues» zu bestimmen. Diese Issues befinden sich dabei in einer erkenntnistheoretisch eigentümlichen Schwebe zwischen spezifischen Themen und allgemeinen Politikfeldern. McCombs und Shaw (ebd.) etwa arbeiten mit den Issues «Foreign policy», «Law and Order», «Fiscal policy», «Public welfare», «Civil rights». Andere Studien arbeiten mit anderen Issues. Beispielsweise operationalisieren Shehata und Strömbäck (2013) Agenden nach den Issues «Unemployment», «Health insurance», «Integration/immigration», «Education», «Elderly care», «Environment», «Taxes», «Health care», «Crime». Keine zwei Studien arbeiten mit denselben Issues; es lassen sich auch kurios anmutende Issues finden, wie etwa «value politics (such as immigration and foreign aid)» bei Hopmann, Vreese und Albaek (2011), bei denen komplett unklar ist, wie sie zustande kommen. Der gängige Umgang mit Issues in Agenda Setting-Forschung ist ein gewichtiges, aber vollends unbeachtetes Problem. Wenn Issues deduktiv a priori definiert werden, um sie in qualitativer Inhaltsanalyse in Agenden zu suchen, dann müsste die Herleitung dieser Issues begründbar sein. Diese Diskussion fehlt aber in der Agenda Setting-Forschung, und die Natur der Issues und deren erkenntnistheoretischer Stand wird ignoriert - der Umgang mit Issues in der Agenda Setting-Forschung ist weitgehend von einer ad hoc-Herangehensweise geprägt. ${ }^{28}$. Ich verzichte auf den Einsatz von Issues, weil sie zu beliebig, da nicht genau und präzise begründbar sind. Stattdessen verwende ich für die Inhaltsanalyse einerseits generalisierte Politikfelder und andererseits spezifische Themen der Untersuchungseinheiten.

$\overline{28} \quad$ Die einzige Ausnahme bildet der Versuch, Issues danach zu unterscheiden, ob sie für gewöhnliche Bürgerinnen und Bürger im Alltag direkt spürbar und von Belang sind («obtrusive» Issues), oder, ob die Issues für sie eher abstrakt bleiben und keinen Bezug zum Alltag haben («unobtrusive» Issues) (Demers u. a. 1989). 
Wenn Politik in den drei Dimensionen Politics (politische Prozesse), Polity (politische Strukturen) und Policy (politische Inhalte) gedacht wird, dann ergeben sich Politikfelder aus dem Versuch, die konkreten Inhalte von Politik nach Bereichen zu bündeln. Politikfelder sind letzlich aber induktive Konstrukte, welche nicht immer trennscharf sind (Blum 2011, S. 14-17). Dennoch ist die Definition von Politikfeldern in einem genüngenden Masse ausgereift, dass es möglich ist - anders als bei Issues - auf eine nicht-willkürliche Kategorisierung zurückzugreifen. Für den in dieser Arbeit verwendeten Katalog an Politikfeldern stütze ich mich auf die Einteilung nach Knoepfel u. a. (2014, S. 623-911). Die verwendeten Politikfelder für die Inhaltsanalyse sind in Tabelle 3 zusammengetragen. Nebst den Politikfeldern sind in Tabelle 3 auch die Politikdimensionen Politics und Polity vermerkt. Diese Politikdimensionen sind zwar keine Politikfelder, aber im Laufe der Erarbeitung des inhaltsanalytischen Vorgehens hat es sich als sinnvoll erwiesen, auch diese zwei zusätzliche Variablen mitzuerfassen. Medien berichten auch über politische Prozesse ohne spezifischen Bezug zu politischen Inhalten (etwa bei personellen Veränderungen bei Parteien, oder bei Analysen von Abstimmungsverhalten, und so fort) wie auch über politische Strukturen, ebenfalls ohne Bezug zu konkreten politischen Inhalten (etwa in Analysen zu Vor- und Nachteilen direkter Demokratie, und so fort). Darüber hinaus können auch parlamentarische Inhalte Bezug zu Politics oder zu Polity und weniger zu konkreten Politikfeldern haben. Von der Logik her stellen die einzelnen Politikfelder das Spektrum der Politikdimension Policy dar. Darum wurde Policy nicht nochmals als separate Politikdimension erfasst.

Wenn auch die Politikfelder deduktiv vorgegeben sind, ist die inhaltsanalytische Arbeit mit ihnen nicht trivial. Die Zuweisung einzelner Politikfelder zu den in Tabelle 2 zusammengefassten Untersuchungseinheiten ist ein qualitativer, sinnverstehender Vorgang, der aber systematisiert werden muss. Die systematische Zuweisung deduktiver Kategorien, wie sie für diese Inhaltsanalyse vorgenommen wurde, kann nach Mayring (2010) als strukturierende Inhaltsanalyse bezeichnet werden. Die genaue Bezeichnung für diese Art der deduktiven qualitativen Inhaltsanalyse ist aber nicht einheitlich; Hsieh und Shannon (2005) beispielsweise nennen dieses deduktive Vorgehen «directed content analysis». Wie auch immer die Bezeichnung ausfällt, das Vorgehen bleibt dasselbe: Die qualitative Kategorisierung des Materials erfolgt anhand expliziter Regeln und idealerweise durch mehrere Personen. Für diese Analyse hat ein Team von drei Personen zuerst gemeinsam die Analyseregeln geübt und anschliessend die Inhaltsanalyse durchgeführt. Die Intercoder-Reliabilität anhand des Krippendorff Alpha-Wertes bewegt sich dabei im Bereich von 0.7, was akzeptabel ist (Krippendorff 2004). Dieser Wert repräsentiert allerdings nur eine Trockenübung und nicht das tatsächliche Vorgehen im Inhaltsanalyse-Team. 
Tabelle 3: Liste der verwendeten Politikfelder, in alphabetischer Reihenfolge.

\begin{tabular}{ll}
\hline Politikfelder & Aussenpolitik \\
& Äussere Sicherheit \\
& Bildungspolitik \\
& Einwanderungs- und Asylpolitik \\
& Finanzpolitik \\
& Forschungspolitik \\
& Gesundheitspolitik \\
& Infrastrukturpolitik \\
& Innere Sicherheit \\
& Internationale Politik \\
& Kulturpolitik \\
& Landwirtschaftspolitik \\
& Medienpolitik \\
& Sozialpolitik \\
& Umwelt- und Raumordnungspolitik \\
& Wirtschaftspolitik \\
\hline Politikdimensionen & Politics \\
& Polity \\
\hline
\end{tabular}

Bei einer Trockenübung weist jede Person separat Kategorien zu, unabhängig davon, wie sicher oder unsicher sie ist. Bei der effektiven Inhaltsanalyse haben wir hochgradig kollaborativ gearbeitet: Nebst regelmässigen Treffen mit Besprechungen von Problemfällen und dergleichen haben wir mit einem interaktiven Online-Codiersystem steten Austausch gepflegt. Damit war es u.a. möglich, bei Unklarheiten und Problemfällen die anderen Teammitglieder in Echtzeit zu benachrichtigen, um eine gemeinsame Lösung zu bestimmen. Dadurch fällt die Reliabilität der deduktiven Inhaltsanalyse höher aus als ein Test auf Intercoder-Reliabilität ohne diese kollaborative Komponente suggeriert.

Der zweite Teil der Inhaltsanalyse ist das induktive bestimmen der spezifischen Themen der Untersuchungseinheiten. Ein induktives Vorgehen stellt andere Anforderungen als ein deduktives, denn die zuzuweisenden Kategorien sind nicht a priori definiert, sondern werden aus den Inhalten herausgearbeitet. Das Herausarbeiten der Themen der Untersuchungseinheiten als Form der qualitativen Inhaltsanalyse wird in der Literatur auf unterschiedliche Art beschrieben. Hsieh und Shannon (2005) nennen dieses Vorgehen «conventional content analysis», weil, so die Autoren, diese Form der qualitativen Inhaltsanalyse die zentrale Form qualitativer Inhaltsanalyse darstellt. Mayring (2010) beschreibt diese Form der Inhaltsanalyse als zusammenfassende Inhaltsanalyse. Der Arbeitsablauf bei dieser zusammenfassenden Inhaltsanalyse ist aber nicht komplett induktiv, sondern auch iterativ-deduktiv. Das bedeutet 
für die durchgeführte Inhaltsanalyse konkret, dass die thematische Zusammenfassung einer Untersuchungseinheit in einem ersten Schritt qualitativ-induktiv stattfindet. Die durch diese induktive Zusammenfassung definierte Kategorie wird anschliessend - und das ist ein zentraler Schritt - mit einer Datenbank aller definierten Kategorien abgeglichen. Falls bisher keine Einträge in diesem Thementopf vorhanden sind, welche die induktiv definierte neue Kategorie bereits abdecken, dann wird der Thementopf um diese neue Kategorie erweitert. Falls aber bereits eine Kategorie im Thementopf vorhanden ist, welche die induktiv definierte neue Kategorie bereits abdeckt, dann wird die im Thementopf bereits vorhandene Kategorie verwendet. Das grundlegende Vorgehen für die qualitative Bestimmung der konkreten Themen ist also für jede einzelne Untersuchungseinheit induktiv, die einzelnen induktiven Arbeitsschritte werden aber darüber hinaus zusätzlich iterativ-deduktiv angereichert. Diese Form der qualitativen Inhaltsanalyse wird im Englischen bisweilen als «thematic analysis» beschrieben (Braun und Clarke 2006; Fereday und Muir-Cochrane 2008). Der Vorteil eines solchen kombinierten induktiven und iterativ-deduktiven Vorgehens ist, dass die Kategorisierung jeder einzelnen Untersuchungseinheit sinngeleitet und reflektiert ist. Für jede Untersuchungseinheit fallen zwei kognitiv anspruchsvolle Schritte an, die induktive Zusammenfassung und der anschliessende Abgleich des Ergebnisses dieser Zusammenfassung mit dem iterativ erarbeiteten Thementopf. Die Kehrseite dieses Vorgehens ist der Aufwand: Der Prozess des induktiven Zusammenfassens, des Konsultierens und ggf. Erweiterns des Thementopfes sowie das häufige und ausführliche Diskutieren im Team erfordert viel Zeit.

Die Inhalte des Parlamentes werden anhand der Parlamentsprotokolle und der eingereichten Vorstösse in toto analysiert, für die Medienstichprobe erfolgt angesichts der aufwändigen qualitativen Inhaltsanalyse aber eine Einschränkung. Nur jene Zeitungsartikel bzw. Beiträge in Sendungen fliessen in die Inhaltsanalyse, welche folgende Bedingungen erfüllen:

- Es werden ausschliesslich Inhalte der journalistischen, redaktionellen Teile berücksichtigt (Anzeigen, Inserate, Unterhaltendes und dergleichen werden nicht berücksichtigt).

- Bei den journalistischen, redaktionellen Teilen müssen Artikel zwei Bedingungen erfüllen, um für die Inhaltsanalyse berücksichtigt zu werden:

- Die Artikel bzw. Beiträge müssen einen expliziten Bezug zu Politik haben.

- Die Artikel bzw. Beiträge müssen einen expliziten Bezug zur Schweiz haben.

Nachdem definiert ist, was die Erhebungs- und was die Untersuchungseinheiten sind, sowie, wie die konkreten Themen und die Politikfelder erhoben werden, gilt es noch, das Wann zu klären, also die zu untersuchenden Zeiträume zu bestimmen. Da eine Totalerhebung aller 
Parlamentsaktivitäten und aller Berichterstattung zum Parlament keine ernsthafte Option ist, arbeite ich mit Stichproben von Sessionen in der Periode der Medienkrise. Konkret untersuche ich die Aktivitäten der Bundesversammlung und der Medien innerhalb dreier Sessionen. Die Eckpunkte zu den drei ausgewählten Sessionen sind in Tabelle 4 festgehalten.

Tabelle 4: Sessionen für die Inhaltsanalyse.

\begin{tabular}{llll}
\hline Sessionsbeginn & Sessionsende & Sessionstage & Sessionstyp \\
\hline 20.09 .1999 & 08.10 .1999 & 13 & Herbstsession \\
19.09 .2005 & 07.10 .2005 & 13 & Herbstsession \\
12.09 .2011 & 30.09 .2011 & 13 & Herbstsession \\
\hline
\end{tabular}

Für die Analyse betrachte ich drei Herbstsessionen, jene von 1999, jene von 2005 und jene von 2011. Jede dieser Sessionen dauert reguläre drei Wochen, bzw. genauer, 13 Tage (In der den ersten zwei Sessionswochen wird von Montag bis Donnerstag getagt, in der dritten Woche von Montag bis Freitag.). 1999 ist ein Jahr, in welchem die Medienkrise noch nicht in vollem Zug ist, sondern sich erst ankündigt. Wie in Abbildung 1 festgehalten ist, sind in dieser Zeit die Werbeeinnahmen bei Printtiteln noch im Aufstieg begriffen, und Pendlerzeitungen sind noch kaum auf dem Markt vertreten ${ }^{29} .1999$ stellt somit einen Zeitpunkt vor der Medienkrise dar, und 2005 und 2011 sind zwei Zeitpunkte, zu denen die Medienkrise jeweils bereits im Gange ist (2005) und sich weiter verschärft (2011). Die Aktivitäten aller drei Sessionen sind über das amtliche Bulletin (Protokolle der Debatten und Abstimmungen sowie der Fragestunden) sowie über Curia Vista (eingereichte Vorstösse) zugänglich. Die Inhaltsanalyse der Parlamentsprotokolle und der Vorstösse erfolgt mittels Totalerhebung; für die drei Sessionen werden also alle Aktivitäten inhaltsanalytisch erfasst.

Der nächste Schritt der Stichprobenbildung betrifft die Tage innerhalb der Sessionen, für welche die noch zu bestimmende Medienstichprobe untersucht werden soll. Eine Minimalvariante diesbezüglich wäre es, die Medienstichprobe nur an genau jenen 13 Tagen pro Session zu ziehen, an denen auch die Bundesversammlung tagt. Eine solche Minimalvariante dürfte aber den journalistischen Umgang mit der Session nicht gerecht werden. Es ist nämlich anzunehmen, dass eine Session am Wochenende vor Sessionsbeginn und am Wochenende nach Sessionsende in den Medien behandelt wird, und, dass während der Session auch an tagungsfreien Tagen über die Session berichtet wird. Darum habe ich den jeweils vollen Sessionszeitraum, inklusive des Wochnendes vor Sessionsbeginn und des Wochenendes nach Sessionsende, als Zeitraum für die Medienstichprobe gewählt, wie in Tabelle 5 zusammengefasst ist.

$29 \quad 20$ Minuten kam im Dezember 1999 als erste Pendlerzeitung auf den Markt. 
Tabelle 5: Zeiträume für die Medienstichproben.

\begin{tabular}{llll}
\hline Beginn & Ende & Tage & Wochentage \\
\hline 18.09 .1999 & 10.10 .1999 & 23 & 15 \\
17.09 .2005 & 09.10 .2005 & 23 & 15 \\
10.09 .2011 & 02.10 .2011 & 23 & 15 \\
\hline
\end{tabular}

Die Auswahl der konkreten Medientitel ist ein heikler Schritt. Mit der konkreten Stichprobe an Medien wollte ich zwei Ziele erreichen. Zunächst habe ich im Vorfeld bestimmt, dass Titel aus Print, Fernsehen und Radio in die Stichprobe einfliessen sollen. Das zweite Kriterium sollte sein, dass nur solche Titel pro Gattung in die Auswahl kommen, welche den Status von Leitmedien haben. Nun gibt es aber keine einheitliche Definition der Kriterien, welche ein Medium zu einem Leitmedium machen. Künzler (2012) argumentiert etwa, dass die Bezeichnung des Leitmediums stark davon abhängt, für welche Zielgruppe das Medium eine leitende funktion haben soll. Pragmatischerweise habe ich solche Titel in die Medienstichprobe aufgenommen, welche in der Deutschschweiz reichweitenstark hinsichtlich politischer Kommunikation sind. Die Stichprobe an Zeitungstiteln und Sendungen ist in Tabelle 6 zusammengefasst.

Tabelle 6: Medienstichprobe pro Jahr, inklusive der Anzahl Zeitungsausgaben bzw. Fernseh- und Radiosendungen pro Stichprobenahr.

\begin{tabular}{llll}
\hline & 1999 & 2005 & 2011 \\
\hline Neue Zürcher Zeitung & 19 & 19 & 19 \\
Tages-Anzeiger & 19 & 19 & 19 \\
Blick & 19 & 19 & 19 \\
20 Minuten & - & 15 & 15 \\
Blick am Abend & - & - & 15 \\
SonntagsBlick & 4 & 4 & 4 \\
SonntagsZeitung & 4 & 4 & 4 \\
NZZ am Sonntag & - & 4 & 4 \\
Echo der Zeit & 15 & 23 & 23 \\
Tagesschau & 23 & 23 & 23 \\
\hline
\end{tabular}

Wenn eine Zelle mit «-» markiert ist, bedeutet das, dass in dem betroffenen Jahr im Zeitraum der Session der betroffene Titel noch nicht existierte. Die Anzahl der Echo der Zeit-Ausgabe ist für 1999 geringer als für 2005 und 2011, weil es 1999 noch keine Wochenendausgaben des Echo der Zeit gab.

Wie in Tabelle 6 ersichtlich ist, ist die Anzahl Zeitungstitel weit grösser als die Anzahl der Fernseh- und Radiosendungen in der Stichprobe; für das Fernsehen ist lediglich die Tagesschau in der Stichprobe, für das Radio lediglich das Echo der Zeit. Das mag auf den ersten Blick als Verzerrung erscheinen, ist in Tat und Wahrheit einigermassen repräsentativ für die Medien- 
landschaft der Schweiz: Bei Fernsehen und Radio werden de facto nur die Kanäle von SRF in der gesamten jeweiligen Sprachregion rezipiert ${ }^{30}$. Die Printzeitungen in der Medienstichprobe sollen eine möglichst grosse Vielfalt aufweisen. Darum sind nebst den Tageszeitungen NZZ, Tages-Anzeiger und Blick auch die Pendlerzeitungen 20 Minuten und Blick am Abend in der Stichprobe, ebenso wie die Sonntagszeitungen SonntagsBlick, SonntagsZeitung und NZZ am Sonntag.

In Tabelle 4 ist zusammengefasst, welche Zeiträume für welche Sessionen erfasst werden, und in Tabelle 6, welche Stichprobentage für die Medienstichprobe. Gemeinsam mit den oben beschriebenen Auswahlkriterien für Artikel in Zeitungen und Sendungsbeiträge in Fernsehen und Radio lässt sich daraus nun die totale resultierende Stichprobe anhand der Anzahl in der Inhaltsanalyse analysierter Untersuchungseinheiten bestimmen. Die Gesamtsrichprobe ist in Tabelle 7 zusammengefasst.

Tabelle 7: Anzahl der für die qualitative Inhaltsanalyse erfassten Untersuchungseinheiten.

\begin{tabular}{llll}
\hline & 1999 & 2005 & 2011 \\
\hline Neue Zürcher Zeitung & 439 & 464 & 596 \\
Tages-Anzeiger & 449 & 460 & 406 \\
Blick & 243 & 193 & 222 \\
20 Minuten & - & 173 & 165 \\
Blick am Abend & - & - & 75 \\
SonntagsBlick & 161 & 110 & 66 \\
SonntagsZeitung & 160 & 123 & 125 \\
NZZ am Sonntag & - & 126 & 177 \\
Echo der Zeit & 146 & 180 & 136 \\
Tagesschau & 156 & 106 & 91 \\
\hline Geschäfte NR & 1113 & 1580 & 1535 \\
Geschäfte SR & 561 & 561 & 695 \\
Fragestunden & 150 & 201 & 210 \\
Vorstösse & 148 & 222 & 316 \\
\hline
\end{tabular}

Wenn eine Zelle mit «-» markiert ist, bedeutet das, dass in dem betroffenen Jahr im Zeitraum der Session der betroffene Titel noch nicht existierte. Die Anzahl Untersuchungseinheiten für den Ständerat sind für 1999 und 2005 bloss aus Zufall gleich.

Die Fallzahlen der finalen Stichprobe für die Medien und die Fallzahlen der Totalerhebung für das Parlament sind, wie in Tabelle 7 zusammengefasst ist, je nach Medium bzw. parlamentari-

30 Ein wenig anders sieht es im Kanton Tessin aus, wo private Radio- und Fernsehstationen einen grossen Teil der Sprachregion erfassen - dies aber nur, weil die italienischsprachige Sprachregion der Schweiz im Wesentlichen nur aus dem Kanton Tessin besteht. Nebst des Tessins wird Italienisch noch in einigen Tälern des Kantons Graubünden dominanterweise gesprochen. 
scher Arena im zweistelligen bis vierstelligen Bereich. Bei dieser Gesamthaft verhältnismässig hohen Fallzahl pro Stichprobenjahr überrascht es nicht, dass auch die Anzahl der erfassten spezifischen Themen verhältnismässig hoch ausfällt: Für 1999 wurden 947 unterschiedliche Themen erfasst, für 2005 deren 1'115 und für 2011 mit 1'252 am meisten. Der Umstand, dass zunehmend mehr Themen erfasst wurden, überrascht nicht, denn 2005 sind mehr Medientitel in der Stichprobe enthalten als 1999, und 2011 nochmals mehr als 2005.

\subsubsection{Leitfadeninterviews}

Die zweite qualitative Datenerhebung als triangulierende Ergänzung der Inhaltsanalyse sind teilstrukturierte Interviews mit Parlamentarierinnen und Parlamentariern sowie mit Journalistinnen und Journalisten. Teilstrukturierte Interviews, oder synonym, Leitfadeninterviews, sind in zweifacher Hinsicht eine sinnvolle Ergänzung der Inhaltsanalyse. Erstens sind die Untersuchungsgegenstände ${ }^{31}$ bei Leitfadeninterviews deutlich andere als bei der Inhaltsanalyse - Triangulation macht dann am meisten Sinn, wenn nicht identisches Datenmatierial mit bloss unterschiedlichen Methoden analysiert wird, sondern, wenn grundlegend unterschiedliches Datenmaterial generiert wird. Zweitens bedeuten Leitfadeninterviews, dass direkt jene Akteure zu Wort kommen, für welche der Gegenstand der vorliegenden Untersuchung nicht ein abstraktes Thema ist, sondern unmittelbarer Teil ihrer Lebenswelt.

Qualitative Interviews können sehr unterschiedliche Formen aufweisen, denen gemein ist, dass, anders als in standardisierten Befragungen, die Interviewpartner prinzipiell offen auf Fragen antworten bzw. frei sprechen und nur ausnahmsweise mit geschlossenen Fragen konfrontiert werden. Es ist dabei üblich, die Fragen für das Interview nicht im Voraus fix zu definieren, sondern auch situativ innerhalb des Gespräches mit (Nach-)Fragen zu reagieren Weiss (1995). Hopf (2004) schlägt vor, die mögliche Vielfalt an qualitativen Interviewmethoden anhand dreier Dimensionen zu typologisieren. Erstens kann die inhaltliche Ausrichtung des Interviews unterschiedlich stark gesteuert werden; eine starke Steuerungsmöglichkeit ist beispielsweise eine rigide Katalogisierung von Fragen, welche in einer bestimmten Reihenfolge im Interview beantwortet werden müssen. Zweitens kann der Gegenstand des Gespräches sehr fokussiert bis sehr breit sein. Sehr fokussierte Gesprächsgegenstände könne beispielsweise Gespräche sein, bei denen ein spezifischer Filmausschnitt besprochen wird. Drittens gilt es zu entscheiden, ob das Ziel des Interviews eher auf aktivem Fragen seitens der Forscherin oder des Forschers liegen soll, oder eher das Ziel ist, dass die Forscherin oder der Forscher eine

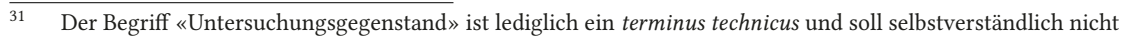
bedeuten, dass meine Gesprächspartnerinnen und -partner Gegenstände sind. 
passive Rolle einnimmt und die Interviewpartner von sich aus aktiv und frei erzählen. Wenn auch diese drei Dimensionen nicht ganz unabhängig voneinander sind, ist das Argument, dass qualitative Interviews ein Spektrum von gesteuert und rigide bis offen und flexibel einnehmen können, wichtig. Hopf (ebd., S. 204) bemerkt, dass eine bestimmte Form der Interviews, welche eine mittlere Position in diesem Spektrum einnimmt, besonders häufig zum Einsatz kommt:

The variants of qualitative interviews that are used particularly frequently are between these extremes and may be described as relatively flexibly applied semistandardized interviews: researchers orient themselves according to an interview guide, but one that gives plenty of freedom of movement in the formulation of questions, followup strategies and sequencing.

Dise sogenannten halbstrukturierten Interviews verbinden einen verhältnismässig hohen Grad der Strukturiertheit Strukturiertheit bezüglich der Diskussionsziele mit der Flexibilität, im Verlaufe des Gespräches situativ zu reagieren und wenn nötig zu vertiefen. Flick (2014, S. 227) schlägt vor, teilstrukturierte Interviews, welche nicht auf die allgemeine Biographie der Interviewpartner abzielen, sondern auf das Fachwissen der Interviewpartner in einem bestimmten Bereich, als Experten- oder Elite-Interviews zu verstehen. Die Gespräche, welche ich für die vorliegende Arbeit durchgeführt habe, fallen in diesen Typus von Interviews: Sinn und Zweck der Interviews ist es, empirische Einsichten zum professionellen Handeln von Parlamentarierinnen und Parlamentariern sowie von Journalistinnen und Journalisten zu erlangen. Teilstrukturierte Interviews im Sinne von Experteninterviews sind gerade im Bereich der - um einen breiten und vielleicht nicht sehr präzisen Begriff zu verwenden - qualitativen Medienforschung sehr verbreitet und erprobt (Riesmeyer 2011).

Für die Interviews habe ich zwischen Juni 2014 und Dezember 2014 insgesamt 26 Gespräche geführt. Die Verteilung der Interviews auf Parlamentarierinnen und Parlamentarier sowie Journalistinnen und Journalisten ist in Tabelle 8 zusammengefasst.

Um die Anonymität der Journalistinnen und Journalisten zu wahren, spezifiziere ich in Tabelle 8 nicht die Zugehörigkeiten zu einzelnen Medien. Als Population für potenzielle Interviewpartnerinnen und -partner kamen nur jene Journalistinnen und Journalisten in Frage, welche für die Medien, welche in der Stichprobe für die Inhaltsanalyse vorkommen, als Bundeshauskorrespondentinnen und -korrespondenten arbeiteten. Auf Seiten des Parlamentes ist die parteiliche Durchmischung befriedigend, wie in Tabelle 9 festgehalten ist. Die Beschreibung «FDP = FDP.Die Liberalen» mag eigentümlich erscheinen, da es eine rekursive Namensauf-
lösung ist. Die Freisinnig-Demokratische Partei (FDP) ist 2009 mit der kleineren Liberalen Partei der Schweiz 
Tabelle 8: Anzahl und Zugehörigkeiten der Interviewpartnerinnen und -partner.

\begin{tabular}{ll}
\hline Zugehörigkeit & Anzahl \\
\hline Nationalrat & 11 \\
Ständerat & 7 \\
Zeitungen & 5 \\
Rundfunk & 3 \\
\hline
\end{tabular}

Bemerkung: Die drei Personen in der Kategorie Rundfunk sind angehörige des öffentlichen Deutschschweizer Rundfunks SRF, bestehend aus Radio und Fernsehen.

Tabelle 9: Parteizugehörigkeiten der interviewten Mitglieder von National- und Ständerat.

\begin{tabular}{ll}
\hline Partei & Anzahl \\
\hline CVP & 5 \\
SP & 5 \\
SVP & 4 \\
FDP & 3 \\
GLP & 1 \\
\hline
\end{tabular}

Legende: CVP = Christlichdemokratische Volkspartei der Schweiz; SP = Sozialdemokratische Partei der Schweiz; SVP = Schweizerische Volkspartei; FDP = FDP.Die Liberalen ${ }^{32}$; GLP = Grünliberale Partei.

Die parteiliche Durchmischung der Interviewpartnerinnen und Interviewpartner seitens des Parlamentes ist zwar befriedigend, aber nicht optimal: Die Parteien sind nicht repräsentativ gemäss ihren Anteilen im National- und Ständerat vertreten (dazu wären grundsätzlich mehr Interviews nötig gewesen), und einige relevante Parteien, etwa die Grüne Partei der Schweiz oder die Bürgerlich-Demokratische Partei, fehlen gänzlich. Aus diesem Grund sind die Interviews nicht geeignet, Aussagen über Gemeinsamkeiten oder Unterschiede der einzelnen Parteien zu machen. Ein letzter Aspekt der Zusammensetzung der Interviewpartnerinnen und Interviewpartner, die Verteilung der Geschlechter, ist in Tabelle 10 zusammegefasst.

Tabelle 10: Geschlechterverteilung der Interviewpartnerinnen und -partner.

\begin{tabular}{lll}
\hline & Frauen & Männer \\
\hline Parlament & 5 & 13 \\
Medien & 4 & 4 \\
\hline
\end{tabular}

In der Stichprobe der Parlamentarierinnen und Parlamentarier sind deutlich mehr Männer als Frauen vertreten. Der Anteil der Gesprächspartnerinnen liegt aber mit knapp 28\% sehr nahe 
am durchschnittlichen Frauenanteil in der Bundesversammlung; 2014 waren im Nationalrat knapp 30\% der Mitglieder Frauen, im Ständerat knapp 20\%. Ich habe unter Einwilligung aller Gesprächspartnerinnen und Gesprächspartner die Interviews als digitale Audiodatei aufgezeichnet. Die aufgezeichneten Interviews wurden anschliessend in einem Team von vier Personen (mich eingeschlossen) zu geschriebenem Text transkribiert. Bei der Transkription von Tonaufnahmen zu Text gibt es unterschiedliche Konventionen. Es gilt beispielsweise, abzuwägen, ob die ganzen Gespräche oder nur bestimmte Ausschnitte transkribiert werden, oder auch, ob wortwörtlich oder leicht paraphrasierend transkribiert wird. Für die Transkription habe ich die Vorschläge von McLellan, MacQueen und Neidig (2003) umgesetzt: Volle Transkription unter Beibehaltung der morphologischen Eigenheiten und syntaktischen Strukturen. Ein solches Vorgehen hat den Vorteil, dass bei der Umwandlung von Ton zu Text so wenig Information wie möglich verloren geht. Der Nachteil ist der hohe zeitliche Aufwand für dieses detaillierte Transkribieren. Bei fünf Interviews wurde mit der Transkribierung zusätzlich eine «Übersetzung» vorgenommen: Fünf Gespräche fanden auf Wunsch der betroffenen Interviewpartnerinnen und -partner in schweizerdeutschem Mundart statt. Diese Gespräche wurden bei der Transkription in Standarddeutsch überführt.

Die Leitfäden für die Interviews sowohl mit Mitgliedern der Bundesversammlung wie auch mit Journalistinnen und Journalisten decken sechs Themenblöcke ab:

1. Präsenz von Medien während der Session allgemein.

2. Umgang mit Medien.

3. Agenda Setting allgemein.

4. Strategisches Handeln.

5. Entwicklung.

6. Normative Bewertung.

Diese Themenblöcke sind inhaltlich nicht trennscharf und in den konkreten Gesprächen bezüglich der Reihenfolge und des Umfangs unterschiedlich. Der erste Themenblock, Präsenz von Medien während der Session allgemein, ist bei allen Gesprächen der sogenannte «Eisbrecher»: $\mathrm{Zu}$ Beginn sollten alle Interviewpartnerinnen und Interviewpartner darüber berichten, wie sie die Präsenz von Journalistinnen und Journalisten während der Session erleben. Bei den Interviews mit Journalistinnen und Journalisten haben diese, logischerweise, über ihren eigenen Berufsalltag berichtet. Der erste Themenblock diente nicht zuletzt dazu, Anknüpfungspunkte für Fragen hinsichtlich der anderen Themenblöcke zu finden. Der zweite Themenblock, Umgang mit Medien, übt vor allem die Funktion der kognitiven Aktivierung aus, durchaus 
im Sinne von Priming (vgl. Unterabschnitt 3.1). Für diesen Themenblock wollte ich von den Parlamentarierinnen und Parlamentariern erfahren, wie sie in ihrer politischen Biographie gelernt haben, mit Medien umzugehen. Die interviewten Journalistinnen und Journalisten sollten hier nicht über sich selber berichten, sondern die Thematik der medialen Erfahrung der Parlamentarierinnen und Parlamentariern aus ihrer Sicht einschätzen, ebenfalls zum Zwecke der kognitiven Aktivierung. Für den dritten Themenblock, Agenda Setting allgemein, bat ich die Interviewpartnerinnen und -partner um ihre Einschätzungen hinsichtlich zweier Agenda Setting-Ströme: Die Auswahl von Themen für die Berichterstattung seitens der Medien sowie die allfällige Beeinflussung des Parlamentes durch die Medien. Für die Auswahl von Themen für die Berichterstattung wurde in den Interviews nach den drei Teilarenen der regulären Geschäfte, der Vorstösse und der Fragestunden unterschieden. Für den allfälligen Einfluss von Medien haben die Gesprächsparterinnen und -partner über dieselben Teilarenen reflektiert, mit dem Unterschied, dass bei regulären Geschäften die Themen institutionellerweise nicht gesetzt, aber eventuell Abstimmungen durch Berichterstattung beeinflusst werden können. Bei diesem Themenblock dreht sich das Gespräch nicht nur darum, $o b$ es solche Beeinflussungen gibt, sondern auch darum, warum es die Beeinflussungen gibt. Der vierte Themenblock, Strategisches Handeln, fokussiert auf die Thematik, was Parlamentarierinnen und Parlamentarier mit Medienpräsenz erreichen wollen sowie, was Journalistinnen und Journalisten mit Berichterstattung über das Parlament erreichen wollen. Beide Akteursgruppen reflektieren dabei ihre eigene Motivstruktur und jene der jeweils anderen Akteursgruppe. Bei dem Themenblock der Entwicklung geht es für alle Interviewpartnerinnen und -partner um die Frage, ob sich rund um die im Laufe des Gespräches an- und besprochenen Dinge etwas verändert hat in den letzten 10 bis 15 Jahren. In dem Themenblock Normative Bewertung schliesslich treffen die Interviewpartnerinnen und -partner Einschätzungen rund um die Frage, ob es, erstens, aus ihrer Sicht Aspekte in dem besprochenen Themenkomplex gibt, welche aus einer demokratischen Perspektive problematisch sind und wie sie, zweitens, die Gesamtsituation des Verhältnisses von Parlament und Medien aus einer demokratischen Perspektive einschätzen.

Nicht alle Themenblöcke der Interviews haben direkten Bezug zu den Forschungsfragen und Hypothesen. Die ersten zwei Themenblöcke haben, wie beschrieben, vor allem eine aktivierende Funktion: Die Interviewpartnerinnen und -partner werden angeregt, sich aktiv in die Wechselwirkungen von Parlament und Medien hineinzuversetzen. Der letzte Themenblock, die normative Beurteilung, ist ebenfalls nicht direkt an die Forschungsfragen und Hypothesen gekoppelt. Die normative Einschätzung ist Teil der Interviews, um jenseits der empirischen Fragestellungen zusätzlich auf einer Metaebene zu reflektieren, wie nah oder wie fern das in 
Unterunterabschnitt 2.2.2 beschriebene normative Modell der prozeduralisierten Volkssouveränität an den normativen Modellen der Parlamentarierinnen und Parlamentarier sowie der Journalistinnen und Journalisten ist (vgl. dazu Unterunterabschnitt 6.2.1).

\subsection{Datenauswertung}

Die Auswertung der Daten erfolgt analog zur Erhebung der Daten in zwei getrennten Schritten. Die mittels qualitativer Inhaltsanalyse erhobenen Daten werte ich mittels quantitativer statistischer Verfahren aus, die Transkripte der teilstrukturierten Interviews hingegen qualitativ. Die genauere Vorgehensart beschreibe ich in den nachfolgenden Unerabschnitten 4.3.1 und 4.3.2. In Unterunterabschnitt 4.3.3 beschreibe ich schliesslich noch kurz, wie die Ergebnisse der separaten Auswertungen für die Besprechung der Ergebnisse zusammengeführt werden.

\subsubsection{Auswertung der Inhaltsanalyse}

Für die quantitative Auswertung der inhaltsanalytisch erfassten Daten bediene ich mich zweier statistischer Verfahren. Zum einen modelliere ich einfache lineare OLS-Regressionen, zum anderen lineare, auf OLS basierende Zeitreihen-Modelle. Das Modell der einfachen OLSRegressionen, welche ich modelliere, hat folgende Form (in Matrix-Notation):

$$
Y=X \beta+\varepsilon
$$

Analog dazu modelliere ich Zeitreihen mit der folgenden Form:

$$
Y_{i, t}=X_{i, t} \beta+\varepsilon_{i, t} ; i=1, \ldots, N ; t=1, \ldots, T
$$

Die so modellierte Zeitreihe ist ein gepooltes OLS-Modell. Auf den ersten Blick mag es überraschen, dass ich keine «konventionelleren» Zeitreihen modelliere, wie etwa ARIMA- oder VAR-Modelle (Hyndman und Athanasopoulos 2013, S. 213-258, 272-275). Der Grund ist die Komplexität der Struktur der inhaltsanalytisch erhobenen Daten. Einfache Zeitreihen sind «zweidimensional»: Sie bestehen aus einer oder mehreren Variablen, deren Werte für unterschiedliche Zeitpunkte im Datensatz enthalten sind. Die Zeitreihe, welche ich verwende, ist aber «dreidimensional»: Nach wie vor werden eine odere mehrere Variablen für unterschiedliche Zeitpunkte erfasst. Diese Variablen werden aber zusätzlich noch für unterschiedliche Einheiten erfasst. Die Daten, mit denen ich für die Zeitreihen arbeite, sind also sogenannte «time-series cross-section»-Daten (Beck 2001). Die Beschreibung dieser Datenstruktur als «time-series cross-section» veranschaulicht die Struktur der Daten. Es handelt sich um Querschnittsdaten, 
welche für mehr als einen Zeitpunkt erhoben werden. Time-series cross-section-, oder abgekürzt, TSCS-Daten sind gängig in der Forschung auf ländervergleichender Ebene, und in diesem Kontext kann gut nachvollzogen werden, was für eine Struktur TSCS-Daten haben. Wenn wir in einem fiktiven Beispiel für die Schweiz, für Deutschland und für die USA vergleichen, wie hoch das BIP und wie hoch die Lebenserwartung im Jahr 2014 war, dann handelt es sich um einen Querschnitt. Wenn wir aber vergleichen, wie hoch das BIP und die Lebenserwartung in der Schweiz, Deutschland und den USA in den Jahren 2012, 2013 und 2014 waren, dann handelt es sich um TSCS-Daten: Bestimmte Variablen werden für bestimmte Einheiten für unterschiedliche Zeitpunkte erfasst. Die Struktur von TSCS-Daten als wiederholter Querschnittserhebungen ist in formaler Hinsicht identisch mit Panel-Daten, obschon bei Panel-Erhebungen die Anzahl der Zeitpunkte tendenziell geringer als bei TSCS-Daten ist (Beck 2006).

Die Modellierungen sind in der Statistikumgebung R durchgeführt (R Core Team 2015). Für die TSCS-Zeitreihen verwende ich zusätzlich das Paket «plm» (Croissant und Millo 2008) innerhalb der Statistikumgebung R. Bei der Berechnung der TSCS-Zeitreihen implementiere ich die von Beck und Katz (1995, 2011) vorgeschlagenen panel-korrigierten Standardfehler, damit die Effekte in den Modellen nicht überschätzt werden. Die inhaltsanalytisch erfassten Daten sind Zählvariablen; wenn eine Untersuchungseinheit einer deduktiven (Politikfeld) und induktiven (konkretes Thema) Kategorie zugeteilt wird, dann erhöht sich die Anzahl dieser Kategorien innerhalb der Erhebungseinheit um je eins. Zählvariablen als abhängige Variablen sind für OLS-Regressionsmodelle aber suboptimal; stattdessen sollten Regressionsmodelle verwendet werden, denen passendere Verteilungsannahmen zugrunde liegen, etwa Poisson- und NegativBinomial-Modelle (Cameron und Trivedi 2013, S. 71-88). Das Problem bei diesen Modellen für Zählvariablen ist, dass für deren Umsetzung als Zeitreihe die Berechnung der panel-korrigierten Standardfehler nicht ohne Weiteres möglich ist. Aus diesem Grund verzichte ich auf die für Zählvariablen optimierten Modelle. Stattdessen transformiere ich die Zählvariablen zu relativen Anteilen, wie dies z.B. Brandenburg (2002) vorschlägt. Die Variablen der Politikfelder und der konkreten Themen kommen in einer Erhebungseinheit unterschiedlich oft vor. Um diese absoluten Häufigkeiten zu relativen Anteilen pro Erhebungseinheit zu transformieren, genügt eine simple Bruchrechnung. Wenn beispielsweise interessiert, welchen Anteil das Politikfeld Aussenpolitik an der gesamten Berichterstattung des Tages-Anzeiger an einem Tag ausmacht, dann muss schlicht die Anzahl der Kategorie Aussenpolitik in der betroffenen Ausgabe durch die Summe aller Politikfelder (d.h., aller codierten Untersuchungseinheiten, in diesem Beispiel aller Artikel) geteilt werden. Mit demselben einfachen Vorgehen lassen sich die absoluten Häufigkeiten der konkreten Themen zu den relativen Anteilen transformieren. 


\subsubsection{Auswertung der Interviews}

Die Auswertung der Interview-Transkripte erfolgt qualitativ mit dem Ziel, in den Transkripten induktive Kategorien zu den einzelnen in Unterunterabschnitt 4.2.2 beschriebenen Themenblöcken herauszuarbeiten. Um diesen Arbeitsablauf übersichtlich zu halten, habe ich die Software MaxQDA (Kuckartz 2015) verwendet. Die Auswertung der Interviews ist somit de facto eine qualitative Inhaltsanalyse, und zwar eine, nach Mayring (2010), strukturierende Inhaltsaalyse.

\subsubsection{Zusammenführen der Ergebnisse}

Der Designansatz der Triangulation beschreibt, wie in Unterabschnitt 4.1 dargelegt, nicht ein einziges konkretes Forschungsdesign, sondern meint eher eine bestimmte erkenntnistheoretische Herangehensweise an empirische Forschung. Damit können die konkreten Forschungsanlagen, welche im Sinne des Paradigmas der Triangulation aufgestellt werden, eine grosse Vielfalt aufweisen. Sie haben aber alle eine Gemeinsamkeit: Triangulation fördert im Mindesten zwei separate empirische Ergebnisse zutage. Das Ziel von Triangulation ist aber nicht, disparate Empirie herzustellen, sondern, einen Untersuchungsgegenstand aus mehreren Winkeln auszuleuchten. Darum ist es unumgänglich, die mittels triangulierenden Verfahren gewonnenen empirischen Ergebnisse zu einem empirischen Gesamtbild zusammenzuführen.

Eine wichtige Entscheidung in diesem Zusammenhang ist, wie Jick (1979) bemerkt, die Gewichtung der einzelnen Ergebnisse. In Unterabschnitt 4.1 erkläre ich, dass ich die Inhaltsanalyse und die teilstrukturierten Interviews als gleichwertig erachte. Folglich gewichte ich auch die Ergebnisse der Datenauswertung nicht unterschiedlich, sondern erachte sie auch in dieser Hinsicht als gleichwertig.

Eine prinzipielle Gleichwertigkeit der empirischen Ergebnisse gibt für sich genommen aber noch nicht vor, wie genau die Ergebnisse miteinander zu verbinden sind. O’Cathain, Murphy und Nicholl (2010) beschreiben einige Vorgehensweisen für das Verbinden der Ergebnisse bei Mixed Methods-Studien in medizinischen Kontexten. Wenn auch die Autoren das wichtige Argument nennen, dass das Zusammenführen der Ergebnisse das eigentliche Triangulieren darstellt, auf dessen Grundlage die Interpretation der Ergebnisse stattfindet, bleiben die Vorschläge für das Zusammenführen der Daten eher allgemeiner Natur. Dies aus dem Grund, dass es keine formalen Prozeduren gibt, um triangulierte Ergebnisse zu einer einzigen Aussage zu vermengen, wie dies beispielsweise bei Metaanalysen möglich ist. Moran-Ellis u. a. (2006) unterscheiden zwei Formen der Zusammenführung der Ergebnisse, «seperate methods, integrated analysis» und «separate methods, separate analysis, theoretical integration». 
Tabelle 11: Methoden zur Prüfung der Hypothesen.

\begin{tabular}{ll}
\hline Hypothese & Methoden \\
\hline H1 & Inhaltsanalyse + Interviews \\
H2 & Inhaltsanalyse + Interviews \\
H3 & Inhaltsanalyse + Interviews \\
H4 & Inhaltsanalyse + Interviews \\
H5 & Inhaltsanalyse + Interviews \\
H6 & Inhaltsanalyse + Interviews \\
H7 & Interviews \\
H8 & Inhaltsanalyse \\
H9 & Inhaltsanalyse + Interviews \\
\hline
\end{tabular}

Bemerkung: Hypothese H7 wird lediglich anhand der Interview-Daten geprüft, Hypothese H8 lediglich anhand der inhaltsanalytischen Daten. Alle anderen Hypothesen werden trianguliert durch eine gleichgewichtete Betrachtung sowohl der Interview-, wie auch der inhaltsanalytischen Daten.

Die Ergebnisse der Triangulation für die vorliegende Arbeit führe ich in der letzteren Variante zusammen: Die Datenauswertung führe ich separat für die inhaltsanalytischen und die interviewbasierten Daten durch und führe erst die Ergebnisse zusammen. Die erste Variante der «integrierten Analyse» meint den Vorschlag, die trianguliert erhobenen Daten gleichzeitig in quasi-explorativer Manier auszuwerten; ein nur leidlich systematischer und kaum reproduzierbarer Vorgang. Da ich die Datenanalysen separat durchführe, besteht die einzige Möglichkeit, sie zusammenzuführen, darin, die Ergebnisse argumentativ zu verbinden und zu reflektieren. Ein leicht verkomplizierender Aspekt in dieser Hinsicht ist der Umstand, dass ich nicht für alle in Unterabschnitt 3.4 formulierten Hypothesen trianguliere. Tabelle 11 fasst zusammen, welche Hypothesen mit welchen Methoden geprüft werden.

Die Hypothesen H7 und H8 werden nicht trianguliert, sondern nur anhand der Interviews bzw. anhand der Inhaltsanalyse geprüft. Das ist nicht mangelnden oder mangelhafen Daten geschuldet, sondern der Natur der Hypothesen. Die Hypothese $\mathrm{H} 7^{33}$ spricht die Motivstruktur der Parlamentarierinnen und Parlamentarier an, welche sich nicht aus den inhaltsanalytisch erfassten Daten ablesen lässt. Für Hypothese $\mathrm{H} 8^{34}$ ist das Gegenteil der Fall, denn sie betrifft die Häufigkeiten, mit denen Journalistinnen und Journalisten über die unterschiedlichen Teilarenen der Bundesversammlung berichten; diese Grössen lassen sich zuverlässig aus den inhaltsanalytischen Daten ablesen.

33 «Parlamentarierinnen und Parlamentarier handeln medialisiert als Mittel zum Zweck zugunsten der Politiklogiken».

34 «Medien berichten am meisten über die traktandierten Geschäfte, weniger über die eingereichten Vorstösse und am wenigsten über die Fragestunden im Nationalrat». 


\subsection{Schwächen des Designs und der Methoden}

Das Forschungsdesign ist, funktional gesehen, Mittel zum Zweck der Beantwortung der Forschungsfragen. Die Aussagekraft der Ergebnisse, welche über die Erhebung und Auswertung der Daten zutage gefördert werden, lässt sich denn auch nur einschätzen, wenn auch die Schwächen des Designs bedacht werden. Das Forschungsdesign gibt in diesem Sinne Aufschluss darüber, in welchem Masse das, was untersucht werden sollte, mit dem, was effektiv untersucht wurde, korrespondiert.

Das Design der vorliegenden Arbeit hat in Betreff der analysierten Stichproben einen bedeutenden Mangel, welcher die Aussagekraft der Ergebnisse mindert. Bei der Inhaltsanalyse erfasse ich die gesamten parlamentarischen Aktivitäten der drei Sessionen, aber nicht die gesamten Aktivitäten der Medien in diesem Zeitraum. Nun macht es natürlich Sinn, nicht alle Medien und alle Medienberichterstattung während der Sessionen zu untersuchen, sondern nur eine Stichprobe. Das Problem mit der gewählten Stichprobe an Medientiteln ist aber, dass ich ausschliesslich Deutschschweizer Medien beachte. Das schafft eine deutliche Asymmetrie: Für die parlamentarischen Aktivitäten während der Sessionen mache ich eine Totalerhebung, und zwar nicht zuletzt, weil eine sprachregionale Trennung innerhalb der Sessionsaktivitäten sinnfrei wäre. Wenn auf Medienseite aber nur Deutschschweizer Titel beachtet werden, ist die damit implizierte Annahme, dass eine Deutschschweizer Stichprobe an Medien als pars pro toto auch für Medien aus der Romandie und dem Tessin gelten kann. Auch wenn diese Annahme empirischen Gehalt haben sollte - wofür ich keine Evidenz vorzuweisen habe - , bleiben die asymmetrischen Daten aus einer handlungstheoretischen Perspektive ein Problem. Es ist nämlich anzunehmen, dass die Interaktionen zwischen Parlamentarierinnen und Parlamentariern und Journalistinnen und Journalisten intensiver innerhalb der Sprachgruppen als zwischen den Sprachgruppen sind. Das bedeutet konkret, dass Deutschschweizer Parlamentarierinnen und Parlamentarier stärker mit Deutschschweizer Journalistinnen und Journalisten interagieren, Westschweizer Parlamentarierinnen und Parlamentarier stärker mit Westschweizer Journalistinnen und Journalisten, Tessiner Parlamentarierinnen und Parlamentarier stärker mit Tessiner Journalistinnen und Journalisten. Diesen Umstand der sprachlichen Segmentierung hat ein Journalist in einem der Interviews ebenfalls angesprochen, als er zu beschreiben versuchte, nach welchen allgemeinen Logiken und mit was für Heuristiken Medienschaffende ihrer Arbeit nachgehen:

Ich würde Mal so sagen, in der Westschweiz kennt man die wenigsten Parlamentarier aus der Deutschschweiz. [...] Weshalb? Weil die Medien natürlich immer 
diejenigen Leute nehmen, die aus ihrem Sprachgebiet kommen. Ist ganz interessant, oder. Unsere Westschweizer Kollegen [...], die nehmen natürlich ihre Leute aus der Westschweiz. [...] Deshalb gibts zum Teil auch eine etwas, eine mediale Verzerrung.

Wenn nun eine Asymmetrie zwischen der Totalerhebung der Parlamentsaktivität und der sprachregional begrenzten Stichprobe der Medien besteht, bedeutet das mit hoher Wahrscheinlichkeit, dass die quantitativ gemessenen Agenda Setting-Effekte unvollständig abgebildet werden, weil ein Teil der Wechselwirkungen mit Medien aus den anderen Sprachregionen stattfindet. Das hat zwangsläufig zur Folge, dass die quantitativ gemessenen Effekte tendenziell zu schwach sind. Bei der Auswahl der Interviewpartnerinnen und -partner habe ich mich ebenfalls nur auf Deutschschweizer Mitglieder des National- und Ständerates sowie auf Deutschschweizer Journalistinnen und Journalisten beschränkt. Auch hier stellt sich die generelle Frage, ob die Erfahrungen und Einschätzung dieser Deutschschweizer Stichprobe eine generalisierbare Aussagekraft besitzen. Das Problem ist aber auf der rein analytischen Ebene nicht akzentuiert wie bei der Inhaltsanalyse, weil die sprachregionale Zugehörigkeit der zwei Gruppen dieselbe ist. Eine ähnlich problematische Konfiguration wie bei der Inhaltsanalyse wäre es gewesen, die Stichprobe an Parlamentarierinnen und Parlamentariern so zu belassen, wie sie ist, die Stichprobe an Journalistinnen und Journalisten aber auf die Romandie und das Tessin auszuweiten.

Das bedeutet aber nicht, dass die Interviews grundsätzlich unproblematisch sind. Qualitative Forschung sieht sich, im Allgemeinen, mit der Grundsatzproblematik konfrontiert, dass die Trennlinie zwischen qualitativen Daten und blossen Anekdoten nicht immer sehr klar ist (Merriam 1995). Bei der Methode der qualitativen Interviews, im Besonderen, kommen noch weitere erschwerende Umstände hinzu, allen voran vielleicht die Reaktivität der Methode (Onwuegbuzie und Leech 2006; Roulston 2010). Diese zwei Gefahren werden im Rahmen des verwendeten Forschungsdesigns in zweierlei Hinsicht eingedämmt. Erstens ist es genau die Stärke eines triangulierenden Designs, die Schwachpunkte einer bestimmten methodischen Herangehensweise durch das Verwenden mindestens einer zweiten methodischen Herangehensweise aktiv zu kompensieren. Zweitens verträgt sich meine erkenntnistheoretische Ambition der durchgeführten Interviews mit der erkenntnistheoretischen Güte von Interviews als Methode. Bei den durchgeführten Interviews handelt sich nämlich, wie in Unterunterabschnitt 4.2.2 beschrieben, um Experteninterviews, und das Ziel der Interviews ist es, Handlungsdynamiken und Motivstrukturen von Akteuren innerhalb dieser überschaubaren Teilsysteme zu erkunden. Das bedeutet, dass die Akteure als Angehörige sehr spezifischer Teilsysteme Auskunft geben über ihr Handeln in ihrer Rolle als Mitglieder dieser sehr spezifischen Teilsysteme. 
Nebst diesen gewichtigeren Aspekten sind einzelne weitere Details im Rahmen des Forschungsdesigns suboptimal, welche aber nicht in einem Masse schwerwiegend sind, dass wegen ihnen das Design an und für sich in Frage gestellt würde. So habe ich ein Teil der Interviews nicht auf Standarddeutsch, sondern auf Schweizerdeutschem Mundart geführt. Das hat einerseits einen leichtem Informationsverlust bei der Transkription der Interviews zur Folge, da das Mundart auf Standarddeutsch «übersetzt» wird. Andererseits, und vielleicht wichtiger, ist der Umstand, dass das individuelle Denken und Empfinden durch die Sprache, welche verwendet wird, geprägt ist - egal, ob Schweizerdeutsch und Standarddeutsch als gleichwertige Sprachen bei bi- oder multilingualen Individuen betrachtet werden (Luna, Ringberg und Peracchio 2008), oder, ob Standarddeutsch als Fremdsprache betrachtet wird (Keysar, Hayakawa und An 2012). Somit ist es etwa möglich, dass das Sprechen auf Standarddeutsch eine systematische Verzerrung bedeutet. Diese Möglichkeit kann ich nicht ausschliessen, aber ich gehe davon aus, dass der verzerrende Effekt, so er existiert, eher klein ist.

Ein weiterer Aspekt, welcher nicht optimal gelöst ist, betrifft die Granularität der Inhaltsanalyse. Wie in Unterunterabschnitt 4.2.1 festgehalten ist, hat die aufwendige qualitative Inhaltsanalyse rund 1'000 unterschiedliche konkrete Themen pro Stichprobenjahr zutage gefördert. Für die quantitative Auswertung dieser qualitativen Daten interessieren aber nicht individuelle Themenkarrieren, sondern aggregiert beobachtbare Effekte. Es wäre darum an und für sich angebracht gewesen, einen weiteren Triangulationsschritt einzuführen: Die qualitative Rekonstruktion einzelner Themenkarrieren. Ein solcher Triangulationsschritt würde eine empirische Brücke zwischen generellen, quantitativ gemessenen Effekten und den individuellqualitativen Einschätzungen der betroffenen Akteure schlagen. Hier spielt aber schlicht das Nullsummenspiel der verfügbaren Ressourcen eine Rolle: Je mehr Triangulationsschritte mit den endlichen für die Forschung verfügbaren Ressourcen durchgeführt werden, desto oberflächlicher fallen die Triangulationsschritte aus. Die Anzahl Triangulationsschritte ist also ein Abwägen von Breite gegen Tiefe der Triangulation - für die vorliegende Arbeit habe ich mich für mehr Tiefe entschieden.

Open Access Dieses Buch wird unter der Creative Commons Namensnennung - Nicht kommerziell 4.0 International Lizenz (http://creativecommons.org/licenses/by-nc/4.0/deed. de) veröffentlicht, welche für nicht kommerzielle Zwecke die Nutzung, Vervielfältigung, Bearbeitung, Verbreitung und Wiedergabe in jeglichem Medium und Format erlaubt, sofern Sie den/die ursprünglichen Autor(en) und die Quelle ordnungsgemäß nennen, einen Link zur Creative Commons Lizenz beifügen und angeben, ob Änderungen vorgenommen wurden.

Etwaige Abbildungen oder sonstiges Drittmaterial unterliegen ebenfalls der genannten Creative Commons Lizenz, sofern sich aus der Abbildungslegende oder der Quellreferenz nichts anderes ergibt. Sofern solches Drittmaterial nicht unter der genannten Creative Commons Lizenz steht, ist eine Vervielfältigung, Bearbeitung oder öffentliche Wiedergabe nur mit vorheriger Zustimmung des betreffenden Rechteinhabers oder auf der Grundlage einschlägiger gesetzlicher Erlaubnisvorschriften zulässig. 


\section{Ergebnisse und Diskussion}

\subsection{Ergebnisse}

Die Ergebnisse zu den einzelnen Hypothesen präsentiere ich in den nachfolgenden Unterabschnitten, gebündelt nach Hypothesen, welche nah beieinander liegen. Für jede Hypothese werden dabei jene Ergebnisse - die quantitativen und/oder die qualitativen - besprochen, welche gemäss der Auflistung in Tabelle 11 für die jeweilige Hypothese verfügbar sind. Für die quantitative Auswertung der Inhaltsanalyse kommen teils Zeitreihenmodelle zum Einsatz, teils einfache lineare Modelle (vgl. Unterunterabschnitt 4.3.1). Um was für ein Modell es sich warum jeweils handelt, wird im Text besprochen. Der Unterschied lässt sich aber auch aus den zusammenfassenden Tabellen Ablesen: Wenn in einer Tabelle die Spalte «SE» («Standard Error»; Standardfehler) vorhanden ist, handelt es sich um ein einfaches lineares Modell. Wenn hingegen die Spalte «PCSE» («Panel-Corrected Standard Error»; panel-korrigierter Standardfehler) vorhanden ist, bedeutet dies, dass es sich um eine Zeitreihe handelt. Alle Zeitreihen verwenden die Zeiteinheit eines Tages.

In die Medienstichprobe fallen, wie in Unterunterabschnitt 4.2.1 beschrieben, eine Reihe Deutschschweizer Titel. Für die quantitative Auswertung arbeite ich nicht mit den Themenagenden auf der Ebene einzelner Titel, sondern aggregiere die ganze Medienstichprobe zu einer Medienagenda. Für diese Aggregation habe ich eine Transformation bei drei Titeln bzw. Sendungen gemacht, damit die aggregierte Medienagenda Sinn macht. Echo der Zeit, die Tagesschau und Blick am Abend erscheinen jeweils abends, das restliche Mediensample frühmorgens. Das bedeutet, banalerweise, dass Echo der Zeit, Tagesschau und Blick am Abend an dem Tag, an welchem sie erscheinen, über diesen Tag berichten. Auf die Bundesversammlung bezogen: Diese drei Titel können dann, wenn sie erscheinen, über das Parlamentsgeschehen an demselben Tag berichten, da der National- und Ständerat morgens und nachmittags tagen. Die anderen Titel in der Medienstichprobe hingegen erscheinen allesamt frühmorgens, sodass sie erst am nächsten Tag über das Parlamentsgeschehen berichten können. Für das Aggregieren der Medienstichprobe ist es darum nötig, alle Titel in der Medienstichprobe zeitlich aneinander anzugleichen. Dazu habe ich vor der Aggregation bei Echo der Zeit, Tagesschau und Blick am Abend einen «Lag», also eine Verzögerung, eingebaut. In der Datenstruktur sind die Agenden dieser drei Titel um einen Tag verzögert, damit vom zeitlichen Bezug her mit den anderen Titeln identisch sind. Das bedeutet beispielsweise, dass eine Ausgabe der Tagesschau von einem Montag mit der Verzögerung zeitlich auf einen Dienstag gelegt wurde, um die zeitliche Logik von Zeitungen zu simulieren. 


\subsubsection{H1 und H2: Medieneinfluss auf Abstimmungsverhalten}

Die ersten zwei Hypothesen betreffen das Abstimmungsverhalten im National- und Ständerat. Abstimmungen zu unterschiedlichen Vorlagen stellen gewissermassen den wesentlichen Moment der Entscheidungsfindung während der Session dar. Ich postuliere, dass das Abstimmungsverhalten sowohl im Nationalrat als auch im Ständerat nicht dadurch beeinflusst wird, dass über die abzustimmenden Geschäfte in den Medien berichtet wird:

- Hypothese H1: Abstimmungen im Nationalrat sind nicht durch die vorangehende Berichterstattung zu den betroffenen Geschäften beeinflusst.

- Hypothese H2: Abstimmungen im Ständerat sind nicht durch die vorangehende Berichterstattung zu den betroffenen Geschäften beeinflusst.

Diese Hypothesen teste ich sowohl mit Daten der Inhaltsanalyse wie auch mit Daten aus den Interviews. Für den inhaltsanalytischen Aspekt betrachte ich Gesamt- und Schlussabstimmungen im National- und im Ständerat. Um eine quantifizierende Annäherung zwischen Abstimmungsverhalten und Berichterstattung zu schaffen, verwende ich ein einfaches lineares Modell. Konkret vergleiche ich für jede Session, ob der Anteil der Ja-Stimmen bei einer Gesamtoder Schlussabstimmung durch den Anteil der Medienberichterstattung zu diesem Geschäft beeinflusst wird. Die Fallzahlen sind insgesamt eher gering, sodass die Ergebnisse als explorative Annäherung, nicht aber als abschliessendes Ergebnis betrachtet werden können. Die Anzahl der erfassten Abstimmungen pro Kammer und Jahr ist in Tabelle 12 zusammengefasst.

Tabelle 12: Anzahl der untersuchten Abstimmungen für 1999, 2005 und 2011.

\begin{tabular}{lll}
\hline Jahr & Kammer & Abstimmungen \\
\hline 1999 & Nationalrat & 41 \\
& Ständerat & 41 \\
\hline 2005 & Nationalrat & 22 \\
& Ständerat & 18 \\
\hline 2011 & Nationalrat & 15 \\
& Ständerat & 21 \\
\hline
\end{tabular}

Falls es einen Einfluss der Berichterstattung auf das Abstimmungsverhalten geben sollte, ist nicht klar, wie dieser aussehen könnte. In einem einfachen linearen Modell wird ein einfacher linearer Zusammenhang geprüft: Mehr Medienberichterstattung bedeutet entweder einen höheren oder einen tieferen Ja-Anteil. Ein solcher linearer Zusammenhang erschöpft das mögliche Spektrum an Effekten aber nicht. So ist es etwa denkbar, dass mehr Berichterstattung bis zu 
einem gewissen Punkt zu weniger Ja-Anteilen führt, also zu knapperen ${ }^{35}$ Abstimmungsergebnissen, um nach diesem Punkt der Sättigung wieder zu mehr Ja-Anteilen zu führen. Um auch die Möglichkeit solcher nicht-linearer Zusammenhänge zu prüfen, vergleiche ich für jede Kammer und für jedes Jahr zwei lineare Modelle: Eines, bei welchem nur der Berichterstattungsanteil den Prädiktor («unabhängige Variable») bildet, und zusätzlich ein lineares Modell mit einer polynomialen Komponente zweiten Grades in Form des quadrierten Berichterstattungsanteils. In beiden Modellen bildet, unverändert, der Ja-Anteil die Antwortvariable («abhängige Variable»). Über die Natur polynomialer Modelle herrscht bisweilen leichte Verwirrung, weil solche Modelle nicht-lineare Zusammenhänge abbilden. Es handelt sich bei polynomialen Modellen aber nach wie vor um lineare Modelle; die abgebildeten Zusammenhänge sind zwar nicht linear, aber das diesen Schätzungen zugrundeliegende Modell ist es (Montgomery, Peck und Vining 2012; Seber und Lee 2012). Um einzuschätzen, welches der Modelle für die jeweilige Kammer und das jeweilige Jahr besser zu den Daten passt, habe ich die Modelle anhand des Schwarz Criterion (G. Schwarz 1978), auch als Bayesian Information Criterion bekannt, verglichen. Die Ergebnisse der Schätzungen sind in Tabelle 13 zusammengefasst.

In der Mehrheit der Fälle hat passt das polynomiale Modell besser zu den Daten als das einfache lineare Modell. Für 1999 ist ein polynomiales Modell in beiden Fällen das passendere, aber für 2005 nur für den Ständerat und 2011 nur für den Nationalrat. Wenn für die Prädiktoren in den Modellen allerdings die p-Werte ${ }^{36}$ als Annäherung für die wahrscheinliche Güte der Modelle betrachtet werden, zeigt sich, dass die Schätzungen nicht überall gleich hohe Güte haben. Die Schätzungen für 1999 und 2005 haben tiefere p-Werte, und für 1999 und 2005 sind auch die Effektstärken grösser als 2011. Die stärksten Effekte sind für 1999 vorhanden, die schwächsten für 2011. Dieses Bild wird auch durch den Anteil der erklärten Varianz pro Modell, dem korrigierten $R^{2}$, gestützt. Die Modelle für 2011 erklären am wenigsten, jene für 1999 und 2005 annähernd gleich viel - bis auf den Ausreisser des Ständerates 2005, bei welchem 0.860 der Varianz durch das polynomiale Modell erklärt wird.

Die Effektstärken der quadrierten Prädiktoren in Tabelle 13 sind intuitiv nicht einfach nachvollziehbar, da es sich um die quadrierten Berichterstattungsanteile handelt. Es ist darum

35 Als Indikator für «Knappheit» verwende ich schlicht den Ja-Anteil bei den Abstimmungen. Da alle untersuchten Abstimmungen mehr als 50\% Ja-Anteil haben, funktioniert dieses Vorgehen. Gäbe es allerdings auch Abstimmungen mit weniger als 50\% Ja-Anteil, müssten die Daten umgewandelt werden, z.B. nach dem Muster $\langle$ Knappheit $=1-$ fa-Anteil $\rangle$.

36 Ich verzichte auf das Markieren von «Signifikanzen» anhand der konventionellen Grenzen von 0.001, 0.01 und 0.05. Das frequentistische Arbeiten mit p-Werten ist grundsätzlich problematisch (J. Cohen 1990, 1994; Zhu 2012), und ein Abstufen der p-Werte in «Signifikanzen» ist schlicht komplett willkürlich. Der p-Wert gibt an, wie wahrscheinlich die beobachteten Daten gegeben des Nullmodells sind (Gelman 2013); den p-Wert in «Signifikanzen» abzustufen, ist widersinnig. 
Tabelle 13: Einfluss des Berichterstattungsumfangs auf das Abstimmungsverhalten 1999, 2005 und 2011.

\begin{tabular}{|c|c|c|c|c|c|}
\hline Jahr & Kammer & Prädiktoren & Schätzung & SE & $\mathrm{p}$ \\
\hline \multirow[t]{4}{*}{1999} & \multirow[t]{2}{*}{ Nationalrat } & Berichterstattungsanteil & -3.598 & 1.042 & 0.001 \\
\hline & & Berichterstattungsanteil $^{2}$ & 9.063 & 2.866 & 0.003 \\
\hline & \multirow[t]{2}{*}{ Ständerat } & Berichterstattungsanteil & -2.950 & 0.738 & 0.000 \\
\hline & & Berichterstattungsanteil $^{2}$ & 6.915 & 1.873 & 0.001 \\
\hline \multirow[t]{4}{*}{2005} & \multirow{2}{*}{ Nationalrat } & Berichterstattungsanteil & -1.089 & 0.426 & 0.019 \\
\hline & & Berichterstattungsanteil $^{2}$ & - & - & - \\
\hline & \multirow[t]{2}{*}{ Ständerat } & Berichterstattungsanteil & -2.990 & 0.416 & 0.000 \\
\hline & & Berichterstattungsanteil $^{2}$ & 5.236 & 1.097 & 0.000 \\
\hline \multirow[t]{4}{*}{2011} & \multirow[t]{2}{*}{ Nationalrat } & Berichterstattungsanteil & -0.893 & 0.481 & 0.088 \\
\hline & & Berichterstattungsanteil $^{2}$ & 1.101 & 1.610 & 0.133 \\
\hline & \multirow[t]{2}{*}{ Ständerat } & Berichterstattungsanteil & -0.341 & 0.396 & 0.399 \\
\hline & & Berichterstattungsanteil $^{2}$ & - & - & \\
\hline
\end{tabular}

Berücksichtigt sind nur Gesamt- und Schlussabstimmungen. Der Berichterstattungsanteil gibt an, welchen Anteil die Berichterstattung zu einem einzelnen Geschäft an der gesamten Berichterstattung zu den Geschäften mit Gesamt- oder Schlussabstimmung ausmacht. Wenn für bestimmte Werte nur «-» angegeben ist, bedeutet das, dass das beste Modell für diesen Fall ein einfaches lineares ohne polynomiale Komponente ist. Das korrigierte $R^{2}$ beträgt 1999 für den Nationalrat 0.204, für den Ständerat 0.265; 2005 für den Nationalrat 0.209 und für den Ständerat 0.860; 2011 für den Nationalrat 0.116 und für den Ständerat -0.013 .

nützlich, die Modelle in visueller Form zu betrachten. Die Modelle für den National und den Ständerat für 1999 sind in Abbildung 5 visualisiert.

Beide Modelle weisen eine $\cup$-Kurve auf. Das bedeutet, dass mit höherem Berichterstattungsanteil der Ja-Anteil zunächst abnimmt, um ca. bei dem Punkt 0.2 des Berichterstattungsanteiles wieder zuzunehmen. Allerdings ist aus Abbildung 5 auch ersichtlich, dass dieser Verlauf im Wesentlichen durch je zwei Punkte bedingt ist. Allein auf dieser Datengrundlage kann nicht behauptet werden, dass es sich um tatsächliche Zusammenhänge handelt und nicht bloss um Effekte, welche durch Ausreisser verursacht sind. Aus den Visualisierungen der Modelle für 1999 wird darüber hinaus ersichtlich, dass, erstens, viele Geschäfte, über welche abgestimmt wird, nur wenig Berichterstattung erhalten. Die meisten Abstimmungen haben, zweitens, klare Mehrheiten in der Grössenordnung von 75\% und mehr Ja-Stimmen. Die zwei Modelle der Abstimmungen im National- und im Ständerat 2005 sind in Abbildung 6 visualisiert.

Für 2005 ist das beste Modell für die Abstimmungen im Nationalrat ein einfaches lineares, jenes für die Abstimmungen im Ständerat ein polynomiales. Für das Modell des Ständerates ist erneut eine $\cup$-förmige Verteilung vorhanden, auch dieses Mal mit einem Wendepunkt um 
Abbildung 5: Zusammenhang zwischen Abstimmungverhalten im National- und Ständerat und dem Umfang der Berichterstattung für 1999.

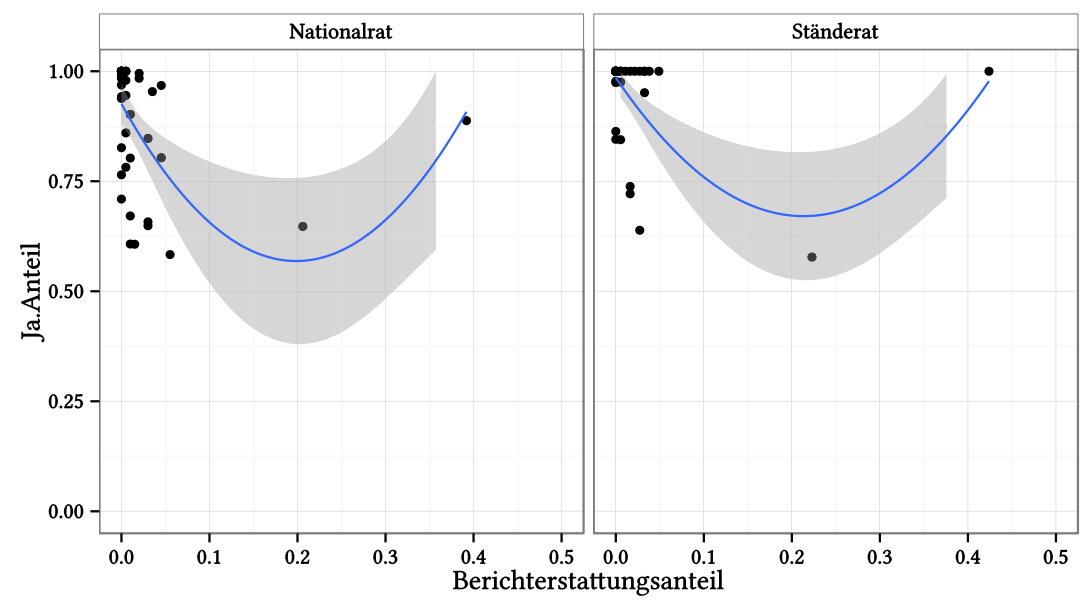

Berücksichtigt sind nur Gesamt- und Schlussabstimmungen. Der Berichterstattungsanteil gibt an, welchen Anteil die Berichterstattung zu einem einzelnen Geschäft an der gesamten Berichterstattung zu den Geschäften mit Gesamt- oder Schlussabstimmung ausmacht. Somit zeigt jeder Punkt an, wie hoch der Ja-Anteil zu diesem Gegenstand bei der Abstimmung ist, sowie, wie hoch der Berichterstattungsanteil dieses Gegenstandes ist. Die Art der Regressionslinie gibt an, welches Modell den besten Fit zu den Daten hat; ein einfaches lineares Modell (gerade Linie) oder ein polynomiales Modell ersten Grades (Kurve). Die graue Fläche ist das 95\%-Konfidenz-Band.

ca. 0.2 bei dem Berichterstattungsanteil. Anders als bei den Modellen für 1999 in Abbildung 5 sind es möglicherweise nicht bloss Ausreisser, welche diesen Zusammenhang hervorbringen. Allerdings ist die allgemeine Fallzahl gering, sodass das Modell auch nicht sehr zuverlässig ist. Dasselbe gilt für das lineare Modell für den Nationalrat, bei welchem der Verlauf des Zusammenhanges sehr stark durch einen Ausreisser geprägt ist. Die Modelle für 2011 schliesslich sind in Abbildung 7 visualisiert.

Für 2011 weist das Modell der Abstimmungen im Nationalrat eine $\cup$-förmige Verteilung auf. Auch bei dieser Kurve liegt der Sättigungspunkt bei ca. 0.2 des Berichterstattungsanteils. Für den Ständerat ist das beste Modell ein einfaches lineares, aber trotzdem kein sehr plausibles: Verhältnismässig viele Abstimmungen sind einstimmig angenommen, ohne, dass Berichterstattung mit dem Ergebnis einhergeht. Handkehrum weisen einige Abstimmungen einen variierenden Grad an Ja-Anteilen auf, und zwar wiederum ohne, dass das Ausmass an Berichterstattung 
Abbildung 6: Zusammenhang zwischen Abstimmungverhalten im National- und Ständerat und dem Umfang der Berichterstattung für 2005.

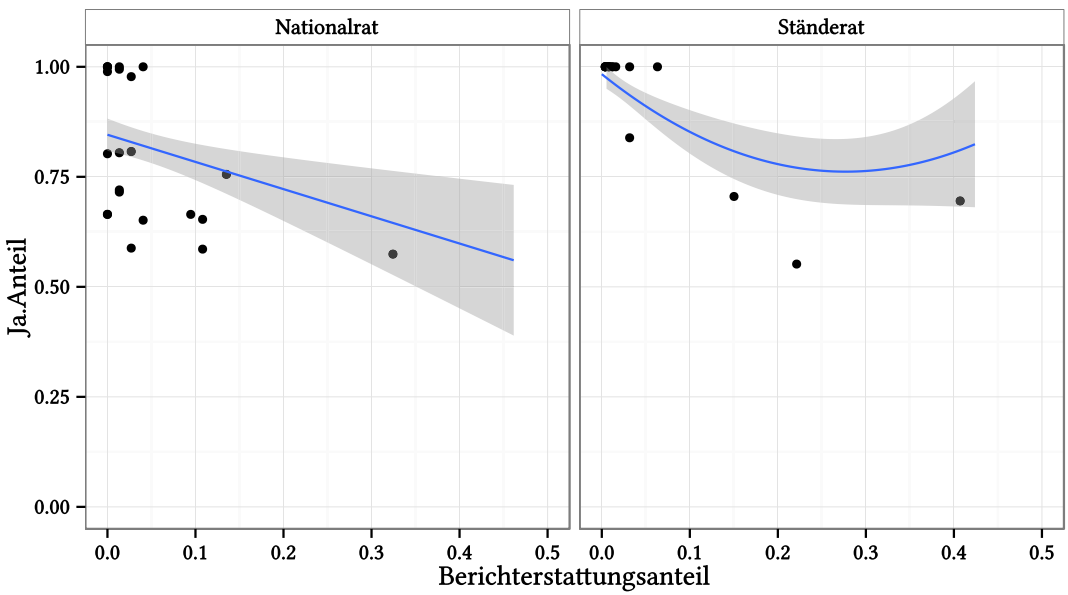

Berücksichtigt sind nur Gesamt- und Schlussabstimmungen. Der Berichterstattungsanteil gibt an, welchen Anteil die Berichterstattung zu einem einzelnen Geschäft an der gesamten Berichterstattung zu den Geschäften mit Gesamt- oder Schlussabstimmung ausmacht. Somit zeigt jeder Punkt an, wie hoch der Ja-Anteil zu diesem Gegenstand bei der Abstimmung ist, sowie, wie hoch der Berichterstattungsanteil dieses Gegenstandes ist. Die Art der Regressionslinie gibt an, welches Modell den besten Fit zu den Daten hat; ein einfaches lineares Modell (gerade Linie) oder ein polynomiales Modell ersten Grades (Kurve). Die graue Fläche ist das 95\%-Konfidenz-Band.

damit einherginge. Angesichts der in Tabelle 13 festgehaltenen geringen Erklärungskraft der Modelle für 2011 und der hohen p-Werte der Schätzungen müssen die Modelle für 2011 als sehr unzuverlässig angesehen werden.

Die quantitativen Ergebnisse zum Einfluss der Berichterstattung auf das Abstimmungsverhalten sind insgesamt eher unklar. Die Fallzahlen sind insgesamt gering, sodass die quantitativen Schätzungen nur grobe Annäherungen ist. Es ist aber auffällig, dass die Mehrheit der Modelle einen $\cup$-förmigen Verlauf zeichnet und, dass bei allen Verläufen der Sättigungspunkt in etwa bei demselben Umfang an Berichterstattung liegt. Sollte es tatsächlich einen solchen Effekt geben, würde das bedeuten, dass mehr Berichterstattung tendenziell zu geringeren Ja-Anteilen führt, um nach einem Sättigungspunkt wieder zu mehr Ja-Anteilen zu führen. Was könnte das aus der Perspektive der betroffenen Akteure bedeuten? Mit einer steigenden Prominenz eines Geschäftes steigen möglicherweise auch die Anreize, nicht konsensuell abzustimmen, aber nur 
Abbildung 7: Zusammenhang zwischen Abstimmungverhalten im National- und Ständerat und dem Umfang der Berichterstattung für 2011.

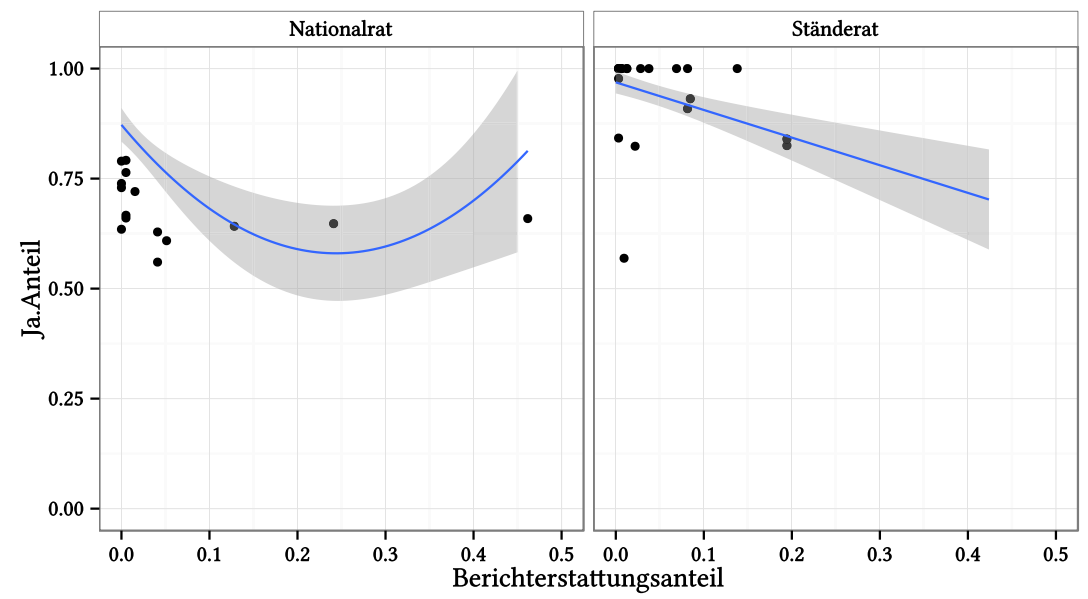

Berücksichtigt sind nur Gesamt- und Schlussabstimmungen. Der Berichterstattungsanteil gibt an, welchen Anteil die Berichterstattung zu einem einzelnen Geschäft an der gesamten Berichterstattung zu den Geschäften mit Gesamt- oder Schlussabstimmung ausmacht. Somit zeigt jeder Punkt an, wie hoch der Ja-Anteil zu diesem Gegenstand bei der Abstimmung ist, sowie, wie hoch der Berichterstattungsanteil dieses Gegenstandes ist. Die Art der Regressionslinie gibt an, welches Modell den besten Fit zu den Daten hat; ein einfaches lineares Modell (gerade Linie) oder ein polynomiales Modell ersten Grades (Kurve). Die graue Fläche ist das 95\%-Konfidenz-Band.

bis zu einem gewissen Grad. Ab dann dreht der Effekt und mit steigender Berichterstattung nehmen die Anreize, konsensuell abzustimmen, wieder zu. Ob ein solcher Effekt ein Handeln nach den Politiklogiken oder nach den Medienlogiken bedeuten würde, ist unklar. Es könnte einerseits sein, dass Parlamentarierinnen und Parlamentarier die unterschiedliche umfangreiche öffentliche Thematisierung der Geschäfte als Signal für die Präferenzen des Prinzipals, also der Bürgerinnen und Bürger deuten und ihr Handeln darum anpassen. Es könnte andererseits aber auch sein, dass sich Parlamentarierinnen und Parlamentarier strategisch nach dem Umfang der Berichterstattung richten, allein, um auf die über die Berichterstattung wahrgenommenen Präferenzen der Medien hinzuhandeln. Für beide Lesarten genügt aber die Analyse des blossen Umfangs der Berichterstattung nicht. Handlungstheoretisch spielt es für die beteiligten Akteure sowohl im Parlament wie auch in den Medien möglicherweise eine Rolle, welche konkreten Geschäfte zur Abstimmung anstehen. 
Diese Vermutung, dass der Einfluss der Berichterstattung auf das Abstimmungsverhalten kein konstanter Effekt ist, sondern, dass der Effekt punktuell und kontigent ist, wird durch die betroffenen Akteure selber in den Interviews geäussert. Ein Mitglied des Nationalrates beschreibt den Einfluss auf das Abstimmungsverhalten wie folgt:

Und insofern, ja, ist es tatsächlich, ist der Einfluss auch auf Parlamentsgeschäfte nicht zu unterschätzen medial. Aber es ist, so direkt, wie ich es jetzt beschreibe, ist es nicht die Regel. Man kann vielleicht von aussen den Eindruck erhalten, weil es ist, es hängt mit dem, was ich vorher gesagt habe zusammen, weil das Gros der Geschäfte geht wie unter dem medialen Radar durch und ist gar nie medial derart präsent.

Ein Mitglied des Ständerates beschreibt die Kontingenz dieses Einflusses als vor allem bei komplexen Geschäften spürbar:

Also bei komplexen Themen bin ich überzeugt, dass das so ist. Dass die Stimme, wie es dann draussen tönt, natürlich zum Teil auch dann zurück in den Ratssaal geht und, dass man da natürlich entweder verunsichert oder dann aber auch, ja als positiv dann betrachtet wird, dass man da sicher ist: Ja, dann sind wir auf dem richtigen Weg. Also eindeutig.

Ein weiteres Mitglied des Ständerates beschreibt, dass nicht nur der blosse Umfang der Berichterstattung eine Rolle spielt, sondern auch, was genau der Zweck der Berichterstattung ist:

Ja, da bin ich überzeugt. Also wenn jetzt irgendein vermeintliches Problem hochgekocht wird, und sich dann eigentlich herausstellt, dass es halb so schlimm ist und man sich fragt, ob das ein Problem war oder nicht.

In dieser Beschreibung des Einflusses der Berichterstattung auf das Abstimmungsverhalten hat eine solche Berichterstattung einen Einfluss, welche nicht bloss hohen Umfang hat, sondern, welche zusätzlich aktiv ein Thema problematisiert und damit die Parlamentarierinnen und Parlamentarier zu einem bestimmten Abstimmungsverhalten bewegt. Eine solche Dynamik von Nachrichtenwellen im Kontext von Problemakzentuieren, oder auch Skandalisierung, wird bisweilen als «Medienhype» beschrieben (Wien und Elmelund-Præstekær 2008, 2009). Dieselbe Dynamik nimmt auch ein weiteres Mitglied des Ständerates wahr: 
Ja, das ist sicher so und ich glaube, da ist der Vorteil des Zweikammersystems in der Schweiz, weil dann reicht etwas, das heute sehr aktuell ist und brennt, dass das heute im Nationalrat diskutiert wird und beschlossen wird und dann 3 Monate später im Ständerat ist dann vielleicht der Pulverdampf etwas verzogen, also ich glaube insofern schon, ja.

Auch Journalistinnen und Journalisten nehmen diese Medienhype-Dynamik im Kontext parlamentarischer Abstimmungen wahr. Ein Journalist hat eine solche Hype-Episode geschildert:

Sicher. Ganz klar. [...] Es gab vor ein paar Jahren mehrere Zwischenfälle mit gefährlichen Kampfhunden; es gab ein, zwei schlimme Unfälle, wo auch Kinder getötet wurden. Und nachher hat dann der Blick eine Art Petition lanciert, welche Unterschriften gesammelt, "Macht jetzt endlich etwas", oder.

In diesem Beispiel hat der Hype zu einer zunächst symbolischen Geste geführt, einer Petition, im weiteren Verlauf dann aber auch zu einem von der Bundesversammlung verabschiedeten Gesetz. Diese Hype-Dynamik kommt, so eine Journalistin, dann zur Geltung, wenn mit dem behandelten Thema eine gewisse emotionale soziale Erwünschtheit einhergeht:

Aber es gibt natürlich schon sehr heikle Themen. Also ich erinnere mich an die Pädophilie-Initiative zum Beispiel. Die wurde ja medial sehr eng begleitet, schon vor der Abstimmung und im Parlament. [...] Also, dass dann unter dem medialen Druck von: Jetzt weiss man genau, jetzt schauen die Medien zu, wie jetzt abgestimmt wird. Dass sich da jemand der Stimme enthält, zufällig nicht im Saal ist oder anders stimmt als er vielleicht sonst gestimmt hätte, das mag durchaus vorkommen.

Aber solche Beeinflussung des Abstimmungsverhalten bleibt, so auch die Meinung der Journalistinnen und Journalisten, punktuell und kontingent. Es ist eher die Ausnahme als die Regel, wie eine Journalistin einschätzt:

Wahrscheinlich gibt es Fälle. Auch das wäre wissenschaftlich zu untersuchen. Wahrscheinlich gibt es Fälle. Aber in der Mehrheit kann ich mir das wenig vorstellen.

Eine notwendige Bedingung für Fälle, in denen Medien einen Einfluss auf das Abstimmungsverhalten ausüben, ist, dass die Berichterstattung nicht nur in einzelnen Titeln vorhanden ist, sondern titelübergreifend behandelt wird. Dazu meint ein Journalist: 
Ja, es muss aber eine grosse Medienöffentlichkeit sein. Ich glaube, es ist jetzt; eine einzige Zeitung, die irgendwas macht, glaube ich weniger, aber wenn vielleicht mehrere Medien über irgendwas berichten.

Die quantitativen und qualtitativen Befunde zeichnen insgesamt ein schlüssiges Bild. Das Abstimmungsverhalten im National- und Ständerat kann durch Berichterstattung beeinflusst werden. Dieser Effekt ist aber nicht universal, sondern punktuell und kontingent. Mit diesen Befunden sind die Hypothesen $\mathrm{H} 1$ und $\mathrm{H} 2$ deutlich widerlegt.

\subsubsection{H3, H4 und H5: Medieneinfluss auf Vorstösse und Fragestunden}

Mit den Hypothesen H3 und H4 postuliere ich, dass sowohl die während einer Session eingereichten Vorstösse wie auch die Fragen in den Fragestunden inhaltlich durch die Berichterstattung der Medien beeinflusst wird. Mit der Hypothese H5 postuliere ich zusätzlich, dass dieser inhaltliche Einfluss der Medien bei den Fragestunden stärker als bei den Vorstössen ist:

- Hypothese H3: Die eingereichten Vorstösse sind durch die vorangehende Berichterstattung beeinflusst.

- Hypothese H4: Die Fragen in den Fragestunden des Nationalrates sind durch die vorangehende Berichterstattung beeinflusst.

- Hypothese H5: Fragen in den Fragestunden sind stärker durch Berichterstattung beeinflusst als die eingereichten Vorstösse.

Die Hypothesen H3, H4 und H5 prüfe ich sowohl quantitativ anhand der auf der Inhaltsanalyse basierenden Daten, als auch qualitativ anhand der Interviews. Für das quantitative Vorgehen arbeite ich dabei mit Zeitreihen wie auch mit einfachen linearen Modellen. Letzteres betrifft nur die Fragestunden, welche nicht geeignet für Zeitreihe-Analysen sind. Dies, weil es pro Session nur zwei Fragestunden gibt, welche jeweils zu Beginn der zweiten und der dritten Sessionswoche stattfinden. Die Fragestunden innerhalb der Zeitreihe für die anderen Teilarenen der Session, also der Geschäfte im National- und im Ständerat sowie der Vorstösse, zu analysieren, wäre darum nicht sinnvoll.

Die Zeitrihenmodelle können auf zwei Arten umgesetzt werden. Die erste Variante ist, die komplette Sessionsdauer, also die kompletten 23 Tage, als eine einzige Zeitreihe zu modellieren. Eine solche Analyse ist aber suboptimal, weil zwar die Medien alle 23 Tage eine Agenda haben, nicht aber das Parlament. Das Parlament tagt für alle drei Stichprobenjahre in der ersten Sessionswoche von Montag bis Donnerstag, in der zweiten Sessionswoche ebenfalls 
von Montag bis Donnerstag, und in der letzten Sessionswoche von Montag bis Freitag. Darum habe ich für die Zeitreihen ein zweites, leicht aufwändigeres Vorgehen gewählt: Ich analysiere pro Session drei separate Zeitreihen, je eine pro Sessionswoche. Für die Hypothese H3 sind die eingereichten Vorstösse die Antwortvariable und die Medien die Prädiktorvariable. Dabei modelliere ich, dass die Medienagenda eines Tages die Vorstösse am folgenden Tag beeinflusst. Zusätzlich baue ich noch die Agenden der Geschäfte des National- und des Ständerates in das Modell mit ein. Mit diesen Kontrollvariablen wird zusätzlich geprüft, ob die Geschäfte des National- und des Ständerates an einem Tag die am Folgetag eingereichten Vorstösse beeinflussen. Die Ergebnisse der drei Zeitreihen für 1999 sind in Tabelle 14 zusammengefasst.

Tabelle 14: Einfluss der Berichterstattung auf eingereichte Vorstösse für 1999.

\begin{tabular}{llrrr}
\hline Zeitraum & Prädiktoren & Schätzung & PCSE & $\mathrm{p}$ \\
\hline 19.09. - 23.09. & Medien $_{t-1}$ & 0.057 & 0.031 & 0.065 \\
& Ständerat $_{t-1}$ & -0.002 & 0.012 & 0.849 \\
& Nationalrat $_{t-1}$ & -0.001 & 0.010 & 0.882 \\
\hline \multirow{2}{*}{ 26.09. - 30.09. } & Medien $_{t-1}$ & 0.059 & 0.047 & 0.207 \\
& Ständerat $_{t-1}$ & -0.003 & 0.020 & 0.868 \\
& Nationalrat $_{t-1}$ & -0.003 & 0.012 & 0.808 \\
\hline \multirow{203.10.\text{-08.10.}}{}{ Medien $_{t-1}$} & 0.000 & 0.012 & 0.997 \\
& Ständerat $_{t-1}$ & 0.012 & 0.011 & 0.269 \\
& Nationalrat $_{t-1}$ & -0.003 & 0.006 & 0.575 \\
\hline
\end{tabular}

Das korrigierte $R^{2}$ beträgt für die erste Woche 0.001 , für die zweite Woche 0.001 und für die dritte Woche 0.000 .

Für 1999 ist das Bild ein sehr deutliches: Die Vorstösse sind weder durch die Agenda Medien, noch durch die Agenden der Geschäfte im National- und Ständerat beeinflusst. Einzig in der ersten Woche 1999 üben die Medien einen sehr kleinen Effekt aus, dessen p-Wert im Vergleich zu den anderen Schätzungen auch für die anderen zwei Wochen Anlass dazu gibt, einen möglichen Effekt zu vermuten.

Die Ergebnisse der Modelle für 2005 sind in Tabelle 15 zusammengefasst. Das Bild ist für die Modelle für 2005 ähnlich wie für jene für 1999. Erneut sind nur in der ersten Woche potenziell relevante Effekte vorhanden. Dieses Mal üben Medien mit 0.333 aber einen sehr starken Effekt aus, was gepaart mit dem tiefen p-Wert die Wahrscheinlichkeit erhöht, dass es sich um einen tatsächlichen Effekt handelt. Auch die Geschäfte des Nationalrates üben in dem Modell für die erste Woche 2005 einen Effekt mit verhältnismässig tiefem p-Wert aus. Der Effekt ist aber, erstens, sehr klein und, zweitens, negativ. Das würde bedeuten, dass Themen, welche 
Tabelle 15: Einfluss der Berichterstattung auf eingereichte Vorstösse für 2005.

\begin{tabular}{llrrr}
\hline Zeitraum & Prädiktoren & Schätzung & PCSE & $\mathrm{p}$ \\
\hline 18.09. - 22.09. & Medien $_{t-1}$ & 0.333 & 0.035 & 0.000 \\
& Ständerat $_{t-1}$ & -0.001 & 0.012 & 0.905 \\
& Nationalrat $_{t-1}$ & -0.019 & 0.009 & 0.030 \\
\hline 25.09. - 29.09. & Medien $_{t-1}$ & -0.003 & 0.019 & 0.857 \\
& Ständerat $_{t-1}$ & -0.003 & 0.009 & 0.753 \\
& Nationalrat $_{t-1}$ & -0.001 & 0.006 & 0.871 \\
\hline 02.10. - 07.10. & Medien $_{t-1}$ & -0.013 & 0.018 & 0.473 \\
& Ständerat $_{t-1}$ & -0.007 & 0.009 & 0.426 \\
& Nationalrat $_{t-1}$ & -0.002 & 0.005 & 0.656 \\
\hline
\end{tabular}

Das korrigierte $R^{2}$ beträgt für die erste Woche 0.029 , für die zweite Woche 0.000 und für die dritte Woche 0.000 .

Gegenstand der nationalrätlichen Debatten sind eher nicht am Folgetag als Vorstoss eingereicht werden. Die Ergebnisse der Modelle für 2011 sind in Tabelle 16 zusammengefasst.

Tabelle 16: Einfluss der Berichterstattung auf eingereichte Vorstösse für 2011.

\begin{tabular}{llrrr}
\hline Zeitraum & Prädiktoren & Schätzung & PCSE & $\mathrm{p}$ \\
\hline 11.09. - 15.09. & Medien $_{t-1}$ & -0.008 & 0.036 & 0.818 \\
& Ständerat $_{t-1}$ & -0.002 & 0.014 & 0.882 \\
& Nationalrat $_{t-1}$ & 0.003 & 0.012 & 0.789 \\
\hline 18.09. - 22.09. & Medien $_{t-1}$ & -0.023 & 0.060 & 0.705 \\
& Ständerat $_{t-1}$ & -0.004 & 0.025 & 0.878 \\
& Nationalrat $_{t-1}$ & -0.001 & 0.014 & 0.921 \\
\hline 25.09. - 30.09. & Medien $_{t-1}$ & 0.003 & 0.016 & 0.842 \\
& Ständerat $_{t-1}$ & 0.004 & 0.008 & 0.626 \\
& Nationalrat $_{t-1}$ & -0.002 & 0.006 & 0.727 \\
\hline
\end{tabular}

Das korrigierte $R^{2}$ beträgt für die erste Woche 0.000 , für die zweite Woche 0.000 und für die dritte Woche 0.001 .

Die Vorstösse sind 2011 für keines der Modelle durch die Inhalte der Medien beeinflusst, ebenso wenig wie durch die Inhalte der Geschäfte des National- und des Ständerates. Gesamthaft also zeigen die Modelle, dass die Vorstösse in der ersten Woche 1999 möglicherweise leicht durch Medien beeinflusst sind, in der ersten Woche 2005 sehr wahrscheinlich durch die Medien beeinflusst sind und in den Wochen für $2011 \mathrm{sehr}$ wahrscheinlich nicht durch Medien beeinflusst sind. 
Um zu prüfen, ob die Fragestunden durch die Agenda der Medien beeinflusst sind, sind unterschiedliche Vorgehensweisen möglich. Die Fragen für die Fragestunden müssen spätestens am Mittwoch in der Woche vor der jeweiligen Fragestunde eingereicht werden. Es wäre somit möglich, einfach zu prüfen, ob die Medienagenda des Mittwochs in der Woche vor den Fragestunden einen Einfluss auf die eingereichten Frage hat. Fragen können aber auch vor diesem Mittwoch eingereicht werden. Darum erachte ich es als sinnvoll, mehr als nur einen Tag der Medienagenda als möglichen Prädiktor für die Fragestunden zu modellieren. Konkret untersuche ich den Einfluss der fünf letzten Tage bis und mit dem Mittwoch vor den Fragestunden. Die fünf Tage sind nicht willkürlich gewählt: Wenn die fünf Tage bis und mit Mittwoch in der Woche vor der jeweiligen Fragestunde analysiert werden, umfassen diese Tage auch das Wochenende, also Samstag und Sonntag. Die Ergebnisse der zwei Modelle für die Fragestunden 1999 sind in Tabelle 17 zusammengefasst.

Tabelle 17: Einfluss der Medienagenda auf Fragestunden für 1999.

\begin{tabular}{llrrr}
\hline & Prädiktor & Schätzung & SE & $\mathrm{p}$ \\
\hline Fragestunde 1 & Medien $_{t-9}$ & 0.285 & 0.053 & 0.000 \\
& Medien $_{t-8}$ & 0.104 & 0.032 & 0.001 \\
& Medien $_{t-7}$ & -0.025 & 0.030 & 0.413 \\
& Medien $_{t-6}$ & -0.027 & 0.049 & 0.580 \\
& Medien $_{t-5}$ & 0.011 & 0.043 & 0.795 \\
\hline Fragestunde 2 & Medien $_{t-9}$ & 0.344 & 0.032 & 0.000 \\
& Medien $_{t-8}$ & 0.042 & 0.025 & 0.094 \\
& Medien $_{t-7}$ & 0.034 & 0.038 & 0.359 \\
& Medien $_{t-6}$ & 0.397 & 0.047 & 0.000 \\
& Medien $_{t-5}$ & -0.018 & 0.040 & 0.653 \\
\hline
\end{tabular}

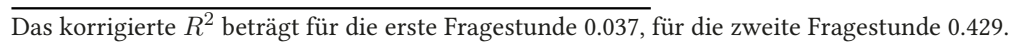

Bei der ersten Fragestunde 1999 üben die Medienagenden der zwei am weitesten zurückliegenden Tage, des Samstags (t-9) und des Sonntags (t-8) starke Effekte bei tiefem p-Wert aus. Für die zweite Fragestunde übt ebenfalls der Samstag vor der Eingabefrist (t-9) einen starken Effekt mit tiefem p-Wert aus, nicht aber der Sonntag. Dafür hat für die zweite Fragestunde die Medienagenda vom Donnerstag ( $t-6)$ vor Ablauf der Eingabefrist einen starken Effekt mit tiefem p-Wert. Die Schätzungen für 1999 legen also nahe, dass bei der ersten Fragestunde die Berichterstattung am Wochenende einen starken Einfluss auf die eingereichten Fragen hat. Bei der zweiten Fragestunde hat nur der Samstag vor Ablauf der Eingabefrist einen Effekt und nicht mehr das gesamte Wochenende. Dafür ist die zweite Fragestunde durch die Medienagenda vom 
Donnerstag (t-6), also einem Tag vor Ablauf der Eingabefrist, beeinflusst. Die Ergebnisse der Modelle für die Fragestunden 2005 sind in Tabelle 18 zusammengefasst.

Tabelle 18: Einfluss der Medienagenda auf Fragestunden für 2005.

\begin{tabular}{llrrr}
\hline & Prädiktor & Schätzung & SE & $\mathrm{p}$ \\
\hline Fragestunde 1 & Medien $_{t-9}$ & 0.087 & 0.041 & 0.032 \\
& Medien $_{t-8}$ & 0.104 & 0.048 & 0.030 \\
& Medien $_{t-7}$ & -0.041 & 0.041 & 0.322 \\
& Medien $_{t-6}$ & 0.002 & 0.038 & 0.949 \\
& Medien $_{t-5}$ & -0.002 & 0.029 & 0.948 \\
\hline Fragestunde 2 & Medien $_{t-9}$ & 0.137 & 0.027 & 0.000 \\
& Medien $_{t-8}$ & -0.006 & 0.037 & 0.878 \\
& Medien $_{t-7}$ & -0.060 & 0.027 & 0.027 \\
& Medien $_{t-6}$ & 0.073 & 0.047 & 0.118 \\
& Medien $_{t-5}$ & 0.014 & 0.032 & 0.670 \\
\hline
\end{tabular}

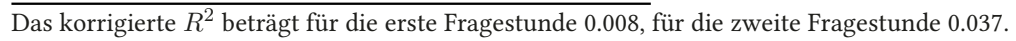

Die Ergebnisse der Modelle für 2005 sind jenen für die Modelle für 1999 ähnlich. Bei der ersten Fragestunde 2005 übt das Wochenende vor Ablauf der Einreichungsfrist, und damit die am weitesten entfernten Tage, die einzigen wahrscheinlich relevanten Effekte aus. Obschon aber die p-Werte verhältnismässig tief sind für den betroffenen Samstag ( $t-9)$ und Sonntag ( $t-8)$, ist der Effekt des Samstags (t-9) deutlich kleiner als in der ersten Fragestunde 1999. Für die zweite Fragestunde übt der Samstag (t-9) vor Ablauf der Eingabefrist einen relevanten, da grösseren Effekt aus, bei tiefem p-Wert. Zusätzlich zeigt sich für den Montag (t-7) angesichts des eher tiefen p-Wertes ein möglicherweise relevanter Effekt. Dieser Effekt ist allerdings schwach und darüber hinaus negativ, was bedeuten würde, dass die eingereichten Fragen eher nicht durch die Medienagenda an diesem Tag beeinflusst sind. Die Ergebnisse der Modelle für die Fragestunden 2011 schliesslich sind in Tabelle 19 zusammengefasst.

Die Ergebnisse für 2011 unterscheiden sich von jenen für 1999 und 2005. Sowohl bei der ersten als auch bei der zweiten Fragestunde 2011 übt die Medienagenda des Mittwochs (t-5), des letzten Tages der Eingabefrist für Fragen, einen möglichen Effekt aus. Dieser ist für die erste Woche allerdings sehr schwach und angesichts des nicht übermässig tiefen p-Wertes zusätzlich unsicher. In der zweiten Woche ist der Effekt deutlich stärker und der p-Wert deutlich tiefer. In der zweiten Woche spielt zudem erneut die Medienagenda des Samstags ( $t$-9) vor Ablauf der Eingabefrist eine Rolle. Der Effekt ist aber sehr klein und negativ, was erneut bedeuten würde, dass die Medienagenda die Inhalte der eingereichten Fragen nicht beeinflusst. 
Tabelle 19: Einfluss der Medienagenda auf Fragestunden für 2011.

\begin{tabular}{llrrr}
\hline & Prädiktor & Schätzung & SE & $\mathrm{p}$ \\
\hline Fragestunde 1 & Medien $_{t-9}$ & 0.007 & 0.030 & 0.818 \\
& Medien $_{t-8}$ & 0.019 & 0.026 & 0.453 \\
& Medien $_{t-7}$ & 0.007 & 0.014 & 0.642 \\
& Medien $_{t-6}$ & 0.012 & 0.024 & 0.627 \\
& Medien $_{t-5}$ & 0.037 & 0.019 & 0.051 \\
\hline Fragestunde 2 & Medien $_{t-9}$ & -0.049 & 0.022 & 0.028 \\
& Medien $_{t-8}$ & -0.014 & 0.025 & 0.572 \\
& Medien $_{t-7}$ & -0.014 & 0.022 & 0.522 \\
& Medien $_{t-6}$ & 0.042 & 0.029 & 0.139 \\
& Medien $_{t-5}$ & 0.114 & 0.028 & 0.000 \\
\hline
\end{tabular}

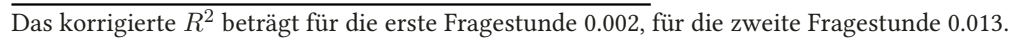

Die quantitativen Analysen zur Prüfung der Hypothesen H3, H4 und H5 zeichnen insgesamt ein konsistentes Bild. Sowohl Vorstösse wie auch Fragestunden werden von den Medien inhaltlich beeinflusst. Die unterschiedlich grossen und unterschiedlich häufigen Effekte deuten darauf hin, dass die Fragestunden stärker von Medien beeinflusst werden als die Vorstösse.

Die Annahme, dass die Inhalte der Berichterstattung in die Inhalte von Vorstössen fliessen, ist für die interviewten Parlamentarierinnen und Parlamentarier eine Selbstverständlichkeit. So meint etwa ein Mitglied des Nationalrates dazu:

Das spielt natürlich eine Rolle. Politiker reagieren natürlich auf aktuelle Themen. Das ist klar. Teilweise natürlich. Es ist nicht so, dass man ausschliesslich auf aktuelle Themen reagiert, aber das spielt natürlich eine Rolle. Politiker lesen auch Zeitung. Die öffentliche Meinung, die öffentliche Wahrnehmung, die öffentliche Befindlichkeit wird ja sehr stark durch Medien geprägt, das ist logisch. Insofern reagieren Politiker natürlich auch darauf. Das ist klar und das sieht man auch.

Wie stark genau eingereichte Vorstösse durch aktuelle Berichterstattung geprägt sind, können die meisten Parlamentarierinnen und Parlamentarier nicht quantifizieren. Ein Mitglied des Ständerates schätzt aber ein, dass die Bedeutung der Berichterstattung für Vorstösse gross ist:

Ich denke, es ist sicher ein Grossteil - ein Grossteil wird lanciert, weil in den Medien etwas stand, etwas thematisiert wurde.

Ein anderes Mitglied des Ständerates erachtet vor allem dein Einfluss der Sonntagsmedien auf die Vorstösse als relevant: 
Ah, das kann man natürlich immer (Lachen), wenn man die Sonntagsmedien liest und nachher in der anderen Woche, was eingegeben wird, das kann man schön nachvollziehen. In den Sonntagsmedien, wenn irgendetwas aufgegriffen wird, dann kommt schon die Reaktion teilweise am Montag

Die Rolle der Medien erachten die Parlamentarierinnen und Parlamentarier bei Fragestunden im Wesentlichen identisch wie bei Vorstössen. Ein Mitglied des Nationalrates etwa erachtet den Einfluss aktueller Berichterstattung bei Fragestunden und bei Vorstössen im Grunde als denselben:

Eben, das ist der gleiche Mechanismus wie die Fragestunden, einfach auf höherem Niveau. Wenn so jemand etwas liest, denkt, das ist ein Problem, und was soll das, und dann macht man einen Vorstoss.

Ein weiteres Mitglied des Nationalrates bringt diesen Einfluss sowohl auf Fragestunden wie auch auf Vorstösse prägnant auf den Punkt und bemerkt zusätzlich, dass sowohl die meisten Fragen wie auch die meisten Vorstösse keine weitreichenden politischen Konsequenzen haben:

Es ist klar, dass die Medien Einfluss auf das Verhalten der Politiker haben, sei es in der Fragestunde oder bezüglich persönlicher Vorstösse, in denen gewisse Ereignisse als relevant bezeichnet werden, auch wenn die meisten von ihnen letztlich im Nichts versanden.

Von den Parlamentarierinnen und Parlamentariern, mit welchen ich Interviews geführt habe, hat keine einzige und kein einziger die Ansicht geäussert, dass die Inhalte der Medien die Inhalte der Fragestunden und der eingereichten Vorstösse nicht beeinflussen. Obschon es für einzelne Parlamentarierinnen und Parlamentarier schwierig ist, genau zu quantifizieren, in welchem Masse die Fragestunden und in welchem die Vorstösse durch Medien beeinflusst werden, ist die geteilte Meinung, dass die Fragestunden relativ gesehen stärker durch Medien beeinflusst sind. In den Worten eines Mitgliedes des Ständerates:

Ja, ich glaube bei den Fragen ist es extrem wichtig, dass es medienrelevante Themen sind - dann kommt man. Sonst versinken die Fragen, also sind die Fragen einfach gestellt und die Antworten zu Kenntnis genommen und finito - oder?

Die Einschätzung, dass die nationalrätlichen Fragestunden stärker durch Medien beeinflusst sind als die Vorstösse ist nicht bloss der «Aussenperspektive» des Ständerates geschuldet. So meint ein Mitglied des Nationalrates zu den Fragestunden: 
Ja die Fragestunde ist ganz stark eine mediale Angelegenheit, weil da geht es am allerseltensten darum, dass man wirklich Infos erhält. Es sind oft ja rhetorische Fragen oder es ist halt die niederschwelligste Möglichkeit, innert kürzester Zeit ein Thema aufzunehmen, das gerade in der Luft liegt, weil der Bundesrat innert einer Woche eine Antwort geben muss.

Gemäss dieser Einschätzung sind Fragestunden darum geeignet, aktuelle Themen aus den Medien aufzunehmen, weil Fragen in den Fragestunden im Unterschied zu eingereichten Vorstössen fast unmittelbar ein Ergebnis zeitigen. Die Auffassung, dass Fragestunden stärker durch Medien geprägt sind als die eingereichten Vorstösse teilt auch eine Journalistin:

Das andere ist vielleicht ein bisschen, ein Vorstoss ist ja, oft braucht es ein bisschen länger als eine Frage. Und da ist es dann vielleicht ein längerer Prozess. [...] Aber die Fragen, bei den Fragen ist es wirklich sehr auffällig, ja.

Eine weitere Journalistin relativiert aber den Einfluss der Medien auf die Fragestunden. Auch, wenn es diesen Einfluss gibt bestehen die Fragestunden nicht komplett aus Themen, welche aktueller Berichterstattung entspringen:

Ich glaube, sie bilden einen Teil der Medienberichterstattung ab, also da kann eine Wechselwirkung stattfinden. Aber zum Teil sind das auch völlig andere Themen, von denen noch nie jemand etwas gehört hat.

Die genaue Art und Weise, wie Inhalte der Medien in die Inhalte von Fragestunden und Vorstössen fliessen, kann unterschiedlich sein. Oftmals ist es, wie aus den obigen Zitaten hervorgeht, individuelle Wahrnehmung seitens der Parlamentarierinnen und Parlamentarier. Es gibt aber auch eine strategischere, parteipolitische Dimension, wie ein Mitglied des Nationalrates mit Führungsfunktion erklärt:

Selbstverständlich. Das gibt es sowieso. Also auf das achtet man täglich. Also meine, unter anderem eine meiner Aufgaben ist es auch, medial das so zu beobachten, damit, wenn ich sehe, da läuft irgendwas, dass ich schaue, dass irgendjemand irgendwo im Umfeld ist, der zum Beispiel zu diesem Thema etwas lanciert.

Die Wahrnehmung von Themen seitens der Parlamentarierinnen und Parlamentarier geschieht nicht nur in Form einseitiger Wahrnehmung von Medieninhalten, sondern auch im direkten Austausch mit Journalistinnen und Journalisten. In einzelnen Fällen kann diese Austausch soweit gehen, dass ein Journalist oder eine Journalistin ein Mitglied des National- oder Ständerates zu einem Vorstoss motiviert, wie ein Mitglied des Nationalrates erklärt: 
Manchmal gibt es auch einen Journalist, der hat das Gefühl, er habe eine Story, oder einen Missstand. Aber es ist noch nicht in der Zeitung gewesen, sondern er fragt jetzt den Politiker. Und dann ruft er an und sagt, «Ah, haben Sie schon gehört, das ist doch so und so», oder «Ich habe herausgefunden, so und so, was halten denn Sie davon?». Und wenn man dann sagt, ja, finde ich eigentlich auch nicht richtig, dann sagt er, «Ja, dann machen Sie jetzt einen Vorstoss?», oder, weil, er würde am liebsten darüber schreiben, dass jemand schon einen Vorstoss macht. Aber weil es noch niemand gemacht hat, wird der Journalist zum Anstifter.

Eine solche Konstellation, in welcher die Journalistin oder der Journalist zum «Anstifter» wird, dürfte eher selten sein, wie ein Journalist bemerkt:

Ist noch heikel. Ich glaube, es gibt es. Ich glaube, das ist auch schon vorgekommen. Ja. Aber also nicht so, dass das in wahnsinniger Regelmässigkeit passieren würde. Ich kann auch nur in dem Sinne von mir sprechen.

Ein Mitglied des Nationalrates meint, dass es solchen Medieneinfluss als «Anstiftung» seitens Journalistinnen und Journalisten sowohl bei Fragestunden wie auch bei Vorstössen gibt:

Ich würde sagen, bei Fragestunden, Vorstössen, sogar bis zu politischen Voten kann das der Fall sein. Aber vor allem bei Fragestunden und Vorstössen, dass er sagt, «Das wäre doch noch Etwas, oder das oder das oder das».

Die meisten Parlamentarierinnen und Parlamentarier, welche eine solche Form des Einflusses beschreiben, nehmen diesen Einfluss wahr, sehen sich selber als diesem nicht direkt ausgesetzt. Nur ein Mitglied des Nationalrates hat direkt erklärt:

Es ist auch schon vorgekommen, dass ich dann gedacht habe: Warum eigentlich nicht? Und das dann tatsächlich gemacht habe.

Ob tatsächlich nur eine derart kleine Minderheit der Parlamentarierinnen und Parlamentarier Fragen oder Vorstösse auf Anregung von Journalistinnen und Journalisten einreicht, oder, ob es sich dei diesem Teilergebnis der Interviews um eine Kombination aus sozialer Erwünschtheit und «Third Person»-Effekt (Davison 1983) handelt, sei dahingestellt. In Betreff der Hypothesen $\mathrm{H} 3, \mathrm{H} 4$ und $\mathrm{H} 5$ deuten die qualitativen Interviews in dieselbe Richtung wie die quantitativen Auswertungen: Sowohl Vorstösse wie auch Fragestunden werden durch die Inhalte aktueller Berichterstattung beeinflusst. Dieser Einfluss scheint bei Fragestunden grösser zu sein als bei eingereichten Vorstössen. Damit können alle drei Hypothesen als gestützt angesehen werden. 


\subsubsection{H6: Politiklogiken und Medienlogiken im Parlament}

In der Hypothese H6 postuliere ich, dass sich Parlamentarierinnen und Parlamentarier in ihrem Handeln stärker nach Politiklogiken denn nach Medienlogiken richten:

- Hypothese H6: Parlamentarierinnen und Parlamentarier handeln stärker nach Politiklogiken als nach Medienlogiken.

Diese Hypothese spricht die in Abbildung 4 visualisierten Präferenzenfamilien der Politiklogiken und der Medienlogiken an. Dabei interessieren zwei Teilmengen: Zum einen die Teilmenge der reinen Politiklogiken, ohne Bezug zu der Präferenzfamilie der Medienlogiken. Andererseits die Teilmenge der Medienlogiken inklusive der Schnittmenge von Medienlogiken und Politiklogiken. Das bedeutet, dass ich in Hypothese H6 postuliere, dass die Teilmenge der reinen Politiklogiken grösser ist als die Teilmenge der reinen Medienlogiken und die Schnittmenge der Politik- und Medienlogiken. Ein erster Schritt, um Hypothese H6 zu prüfen, ist, die Ergebnisse der Hypothesen H1 bis H5 in einer miniaturisierten Pseudo-Metaanalyse zu aggregieren. Zu diesem Zweck fasse ich in einem ersten Schritt die Ergebnisse der Hypothesen H1 bis H5 in Tabelle 20 zusammen.

Tabelle 20: Zusammenfassung der Hypothesen H1 bis H5.

\begin{tabular}{llll}
\hline \multirow{2}{*}{ Hypothese } & \multicolumn{3}{c}{ Ergebnis } \\
\cline { 2 - 4 } & quantitativ & qualitativ & total \\
\hline H1 & 0.3 & 0.2 & 0.25 \\
H2 & 0.3 & 0.2 & 0.25 \\
H3 & 0.6 & 0.9 & 0.75 \\
H4 & 0.8 & 0.9 & 0.85 \\
H5 & 0.7 & 0.6 & 0.65 \\
\hline
\end{tabular}

Die Werte geben an, für wie wahrscheinlich ich die Gültigkeit der Hypothesen gegeben der Evidenz halte. 0 bedeutet, dass ich die jeweilige Hypothese mit der jeweiligen Evidenz als sicher ungültig erachte; 1 bedeutet, dass ich die jeweilige Hypothese mit der jeweiligen Evidenz als sicher gültig erachte.

Die Zusammenfassung in Tabelle 20 mag auf den ersten Blick etwas ungewöhnlich erscheinen. Anstatt wie üblich einfach zu deklarieren, ob eine Hypothese bestätigt oder widerlegt ist, gebe ich an, für wie wahrscheinlich ich es halte, dass die jeweilige Hypothese gegeben der Evidenz, also der Ergebnisse der quantitativen sowie der qualitativen Analysen, ist. Eine solche Beurteilung von Hypothesen ist rationaler als eine dichotome Einteilung nach sicher bestätigt und sicher widerlegt: Empirische Forschung, egal, ob quantitativ oder qualitativ in 
der Auswertung, bedeutet letztlich immer, dass ein Teil der Realität anhand einer Stichprobe geprüft wird. Die erkenntnistheoretische Frage, wie stark diese Stichprobe mit der Realität korrespondiert ist immer eine, so meine ich, probabilistische, nicht eine deterministische.

In den Hypothesen $\mathrm{H} 1$ und $\mathrm{H} 2$ postuliere ich, dass weder der National-, noch der Ständerat in Betreff des Abstimmungsverhaltens durch aktuelle Berichterstattung in den Medien beeinflusst werden. Sowohl quantitativ, stärker noch qualitativ zeigt die Evidenz aber stark in die umgekehrte Richtung, dass nämlich beide Kammern in ihrem Abstimmungsverhalten durch Berichterstattung beeinflusst werden, wenn auch nicht universal und permanent. Mit den Hypothesen H3 und H4 postuliere ich, dass sowohl die Vorstösse wie auch die Fragestunden durch aktuelle Berichterstattung in den Medien beeinflusst werden. Sowohl die quantitative als auch die qualitative Evidenz spricht deutlich für beide Hypothesen, und darum gehe ich entsprechend deutlich davon aus, dass beide Hypothesen Gültigkeit ${ }^{37}$ besitzen; für Hypothese H4 zu den Fragestunden erachte ich die Evidenz als ein wenig stärker, da die quantitativ vorgefundenen Effekte deutlicher sind. Weniger deutlich schätze ich die Hypothese H5 ein. Die quantitative Evidenz ist insofern deutlich, als die Effekte bei den Modellen für die Fragestunden deutlich stärker sind. Allerdings kann das durchaus der Natur der Modelle geschuldet sein: Mit den Zeitreihen modelliere ich ganz explizit nur Zusammenhänge auf der Ebene t-1, für die einfachen linearen Modelle zu den Fragestunden gehe ich aber mehrere Zeitpunkte zurück. Die qualitative Evidenz zu Hypothese H5 spricht an und für sich deutlich für die Hypothese, aber der Umfang der Evidenz ist sehr gering - die allermeisten Interviewpartnerinnen und -partner haben keine Meinung in Kontext der Hypothese H5.

Was bedeutet diese Einschätzung nun für die Hypothese H6? Mit den ersten zwei Hypothesen behaupte ich Handeln nach Politiklogiken, und die Wahrscheinlichkeit, dass diese Hypothesen gültig sind, erachte ich mit je 0.25 als tief. Die Hypothesen H3 und H4 postulieren Handeln nach Medienlogiken ${ }^{38}$, und gegeben der Evidenz erachte ich die Wahrscheinlichkeit, dass diese Hypothesen Gültigkeit haben, als sehr hoch. Hypothese H5 hat nicht unmittelbare Relevanz für Hypothese H6, da Hypothese H5 lediglich aus einem Vergleich aus den Hypothesen H3 und H4 besteht. Die Wahrscheinlichkeiten für die Hypothesen H1 bis H4 in Tabelle 20 lassen sich aber recht einfach zu einer Wahrscheinlichkeit, dass Hypothese H6 gültig ist, aggregieren. Die Wahrscheinlichkeit, dass Parlamentarierinnen und Parlamentarier stärker nach reinen Politiklogiken als nach Medienlogiken handeln, beträgt demgemäss:

37 Ich spreche von der «Gültigkeit» der Hypothesen, um den etwas bedeutungsschwangeren Begriff «Wahrheit» zu vermeiden.

38 Ob dieses Handeln nach Medienlogiken eine Schnittmenge mit Politiklogiken darstellt der nicht ist Gegenstand von Hypothese H7. 


$$
\frac{0.25+0.25+(1-0.75)+(1-0.85)}{4}=0.225
$$

Die Wahrscheinlichkeit, dass Hypothese H6 gültig ist, beträgt angesichts der Hypothesen $\mathrm{H} 1$ bis $\mathrm{H} 4$ also lediglich 0.225. Ist ein solch tiefer Wert als Ergebnis einer simplen, formalen Aggregation schon plausibel? Dass der Wert derart tief ist, mag zunächst verwirren. Es müssen aber im Mindesten zwei Dinge zu dieser tiefen Wahrscheinlichkeit bedacht werden. Erstens bedeutet diese Einschätzung der Wahrscheinlichkeit nicht, dass die Parlamentarierinnen und Parlamentarier mit einer Wahrscheinlichkeit von (1 - 0.225) ausschliesslich nach den Medienlogiken handeln. Die Wahrscheinlichkeit von 0.225 betrifft nur die Einschätzung, dass Parlamentarierinnen und Parlamentarier ausschliesslich nach den Politiklogiken handeln. Die Wahrscheinlichkeit (1 - 0.225) enthält also sowohl die Wahrscheinlichkeit, dass Parlamentarierinnen und Parlamentarier ausschliesslich nach Medienlogiken handeln, als auch die Wahrscheinlichkeit, dass sie nach der Schnittmenge von Politiklogiken und Medienlogiken handeln. Zweitens fokussiere ich mit der vorligenden Arbeit auf das Parlamentsgeschehen während Sessionen. Ich gehe nicht davon aus, dass eine geschätzte Wahrscheinlichkeit von 0.225 auch für die Parlamentsarbeit ausserhalb der Sessionen zutrifft, also für die Kommissionsarbeit - für Kommissionsarbeit würde ich die Wahrscheinlichkeit, dass Parlamentarierinnen und Parlamentarier ausschliesslich nach Politiklogiken handeln, deutlich höher ansetzen. Dies sehen im übrigen auch die Parlamentarierinnen und Parlamentarier selber so, wie eine Einschätzung eines Mitgliedes des Nationalrates illustriert:

Nein. Das darf nicht sein. Das wäre falsch, weil bei den Medien ist der Journalist nicht dabei bei der Kommission, die 25 Leute umfasst, wo die Sache intensiv und wirklich sachlich vorgesprochen wird, durchgesprochen wird, wo Anträge gestellt werden, wo man Kompromisse findet.

Wenn diese zwei Aspekte bedacht werden, ist eine geschätzte Wahrscheinlichkeit von 0.225, dass Hypothese H6 gültig ist, plausibel.

\subsubsection{H7: Ziele medialisierten Handelns im Parlament}

Mit der Hypothese H7 postuliere ich, dass Parlamentarierinnen und Parlamentarier strategisch medialisiert handeln, um damit auf Politiklogiken hinzuarbeiten:

- Hypothese H7: Parlamentarierinnen und Parlamentarier handeln medialisiert als Mittel zum Zweck zugunsten der Politiklogiken. 
Diese Hypothese postuliert also, dass jene in Abbildung 4 dargestellte Schnittmenge zwischen der Präferenzfamilie der Politiklogiken und der Präferenzfamilie der Medienlogiken die gesamte Schnittmenge zwischen Medien und Parlament ausmacht. Diese Hypothese prüfe ich qualitativ anhand der Interviews. Dabei fokussiere ich auf drei Aspekte aus den Interviews:

- Für den in Unterunterabschnitt 5.1.1 beschriebenen wahrscheinlichen Einfluss der Medienberichterstattung auf das Abstimmungsverhalten vertiefe ich die Gründe für diesen Einfluss.

- Für den in Unterunterabschnitt 5.1.2 beschriebenen wahrscheinlichen Einfluss der Medienberichterstattung auf die Vorstösse sowie auf die Fragestunden vertiefe ich die Gründe für diesen Einfluss.

- Zuletzt analysiere ich die Argumente und Erklärungen der Parlamentarierinnen und Parlamentarier zum Interview-Themenblock der Ziele, welche sie mit Medienpräsenz verfolgen.

Die Motivstrukturen der Parlamentarierinnen und Parlamentarier im Kontext des Medieneinflusses bei Abstimmungen werden, wie in Unterunterabschnitt 5.1.1 beschrieben, teilweise geschildert als Beugen vor oder Mitmachen bei einer medialen Welle der akzentuierten Problematisierung emotional aufgeladener Themen - Parlamentarierinnen und Parlamentarier reagieren auf Medienhypes. Ein Mitglied des Ständerates beschreibt das Handeln im Kontext solcher Medienhypes als die Reaktion auf einen spürbaren Druck am Beispiel einer Volksinitiative, welche eine Verschärfung des Berufsverbotes für pädosexuelle Personen forderte:

Nehmen Sie die Pädophilen-Initiative, über die wir jetzt gerade abgestimmt haben. Also da wurde im Nationalrat sogar die Ja-Parole abgegeben am Schluss. Und da wird dann irgendwie, obwohl das Parlament selber schon das Gesetzt geändert hat, und obwohl klar war, dass das gegen das Verhältnis, alle Moral, alles was festgehalten war, Grundprinzipien, würde ich sagen, der Verfassung verstösst. Da gibt es Leute, die getrauen sich dann nicht mehr... also ja, wenn du dagegen bist, dann bist du, schützt du die Pädophilen und ich weiss nicht was, oder du bist selber noch einer. Und der Druck ist dann gross.

Ein weiteres Mitglied des Ständerates beschreibt die Situation rund um Medienhypes als «Aktivismus» am Beispiel der Unfälle mit Kampfunden:

[A]ls dieser tödliche Unfall passiert ist, so tragisch wie das ist, weil Kampfhunde haben ein Kind zu Tode gebissen, das hat ja nachher einen unglaublichen Aktivismus gegeben. Und ich behaupte, der Unfall kann noch heute passieren, trotz 
den Massnahmen, die man gemacht hat, den Kursen, welche die Hundehalter machen und das ganze Theater da, kann immer noch passieren. Das sind tragische Einzelfälle, die nie mit keinem Gesetz zu vermeiden sind. Nie und nimmer.

Diese Einschätzung ist darum bemerkenswert, weil der gesetzgeberische Prozess, der durch den Medienhype ausgelöst wurde, das eigentliche Problem nicht gelöst hat. In diesem Beispiel handelt es sich also um ein Hype-induziertes Handeln, welches keinen Bezug zu den Politiklogiken hat. Medienhypes als Beeinflussung des Abstimmungsverhaltens dürften aber die Ausnahme bilden. Aber auch bei Routine-Berichterstattung spüren die Parlamentarierinnen und Parlamentarier einen gewissen Druck. Ein Mitglied des Ständerates beschreibt dies als Verunsicherung, wenn die eigene Position nicht mit der wahrgenommenen Position der Medien übereinstimmt:

Dass die Stimme wie es dann draussen tönt, natürlich zum Teil auch dann zurück in den Ratssaal geht und dass man da natürlich entweder verunsichert oder dann aber auch, ja als positiv dann betrachtet wird, dass man da sicher ist: Ja, dann sind wir auf dem richtigen Weg.

Dieses Mitglied des Ständerates begründet diese Orienterungsfunktion, welche die Medien für Parlamentarierinnen und Parlamentarier haben, damit, dass Medien als Treiber der Meinung der Bürgerinnen und Bürger vermutet werden:

Also die Medien haben eine recht, das darf man nicht unterschätzen, haben eine gewaltige Macht. Und die können natürlich dann schon, vor allem dann im Volk Stimmung machen, dass das Volk aufbegehrt, dass das Volk natürlich dann nach irgendetwas, ja sage jetzt einmal schreit. Oder über die Medien dann wieder, sie machen dann gezielte Umfragen, bringen vielleicht nur das, was sie eben hören wollen oder eben diese Richtung.

Es ist aber nicht so, dass die Parlamentarierinnen und Parlamentarier die Inhalte und Meinungen der Medien direkt gleichsetzen mit den Präferenzen der Bürgerinnen und Bürger. Ein weiteres Mitglied des Ständerates fasst diesen Aspekt mit dem Argument zusammen, dass es wichtig sei, die Inhalte der Medien zu reflektieren und sie nicht vorschnell mit der Volksmeinung gleichzusetzen:

Man muss einfach aufpassen, dass man dann nicht schon interpretiert: Das ist die Volksmeinung, wenn es schon in der Zeitung steht. Sondern da muss man analysieren: Warum gibt es diese Meinung? 
Dieses Mitglied des Ständerates erklärt denn auch, dass ein allfälliger Einfluss der Medien auf das Abstimmungsverhalten auch das Ergebnis eines Meinungsbildungsprozesses seitens der Parlamentarierinnen und Parlamentarier sein kann:

Und dann sind erste Rückmeldungen aus den Medien, die man kritisch betrachten muss: Woher kommen sie? Warum kommen sie so? Haben die die Ausgangslage identisch gehabt wie wir, dass sie zu diesem Ergebnis kommen? Oder hatten sie eine andere Ausgangslage, um zu diesem Ergebnis zu kommen? Das finde ich eigentlich hilfreich für meine Meinungsbildung.

Ein solcher Mechanismus ist gewissermassen das Gegenteil eines Handelns unter dem Druck von Medienhypes. Das Mitglied des Ständerates beschreibt hier, dass Rückmeldungne aus den Medien zu Geschäften dazu dienen können, neue Perspektiven und Argumente einzubringen. In diese Sinne ist ein Einfluss der Medien auf Abstimmungsverhalten die Folge erweiterter Reflexion der Themen, weil über die Medien neue Argumente in die individuelle Abwägung einfliessen. In extremis würde das bedeuten, dass Medien ein Korrektiv darstellen zu dem parlamentarischen «Group think», also zu der Tendenz, dass Gruppen oft im Sinne gruppendynamischer kognitiver Verzerrungen zuungunsten rationaler Entscheidungsfindung handeln (Janis 1982, S. 174-197).

Die Motivstrukturen der Parlamentarierinnen und Parlamentarier sind hinsichtlich des Medieneinflusses auf eingereichte Vorstösse und Fragen für die Fragestunde gemischt. Zum einen wird betont, dass jene Themen aufgegriffen werden, welche man selber aufrichtig als wichtig und einer Problematisierung wert erachtet. Ein Mitglied des Nationalrates erklärt das folgendermassen:

Das kann mir auch passieren. Ich meine, vielleicht, ist ja nicht ausgeschlossen, dass man auf ein ernsthaftes Problem stösst, auf das man vorher nicht gestossen ist. Also irgendwie müssen wir ja auf unsere Anregungen kommen, wie man das Land noch besser aufstellen könnte.

Zum anderen erklären alle Parlamentarierinnen und Parlamentarier aber auch, dass im Mindestens auch der individuelle Wunsch nach Profilierung eine Rolle spielt, wenn nicht sogar eine dominante. Ein Mitglied des Nationalrates beschreibt dies mit den Worten:

Also, sehr viele Vorstösse sind Reaktionen auf Geschichten in den Medien oder werden deponiert, damit dann Medienarbeit gemacht werden kann. Zur eigenen Profilierung, aber teilweise auch für die Sache. 
Bei dieser kurzen Beschreibung ist bemerkenswert, dass es zunächst um die eigene Profilierung geht, sekundär auch um die Sache an sich. Auf die Frage, ob eher die inhaltliche Überzeugung ob der politischen Relevanz oder das Streben nach medialer Präsenz für das Aufgreifen von Themen aus den Medien wichtig ist, antwortet ein weiteres Mitglied des Nationalrates:

Es ist sicher immer beides. Es ist immer beides. Also das muss man ganz klar wissen. Es soll niemand, wenn man ausschliesslich aus fundamentalistisch politischen Gründe Vorstösse machen würde, dann hätten wir 90 Prozent weniger Vorstösse.

In dieser Einschätzung fällt das Verhältnis nur politisch motivierter Vorstösse zu solchen Vorstössen, welche zusätzlich den einreichenden Parlamentarierinnen und Parlamentariern zu medialer Resonanz verhelfen sollen, sehr deutlich aus. Ein weiteres Mitglied des Nationalrates schätzt die Situation sehr ähnlich ein und argumentiert, dass Vorstösse auf inhaltlicher Ebene grundsätzlich wenig Substanz haben, da die eigentliche inhaltliche Arbeit in den Kommissionen stattfinde:

Die wirklich relevanten Möglichkeiten, sich einzubringen, sind überhaupt nicht via persönliche Vorstösse. Das sind Anträge in der Kommission und dann wirklich konkrete Gesetzesänderungen in einer Revisionsdebatte. Ich behaupte, die Vorstösse haben wirklich eine untergeordnete Relevanz. Es gibt Ausnahmen. Aber in der Regel. Und trotzdem kommen sie so zahlreich. Das hat, das ist praktisch zu $80 \%$ mindestens mit den Medien zu erklären und das ist dann nicht nur die Medien, die berichten und man macht Vorstösse, sondern es ist halt auch oft so, dass jemand findet: Jetzt muss ich wieder einmal ein bisschen präsent sein und am besten mache ich einen Vorstoss, versuche das einem Journalisten zu verkaufen, zu verklickern, dass es interessant sei.

Auch gemäss dieser Einschätzung haben Vorstösse das primäre Ziel, öffentliche Resonanz für die diese Vorstösse einreichenden Parlamentarierinnen und Parlamentarier zu erzeugen. Auch die Fragestunden operieren nach ähnlichen Logiken gemäss eines Mitgliedes des Nationalrates:

Das ist etwas wo jetzt, bedient man die News, also man bedient die Journalisten in der Fragestunde auch. Man stellt etwas, wo gerade in den Zeitungen kommt bzw. etwas das gerade brennt.

Die Fragestunden beurteilt ein weiteres Mitglied des Nationalrates als auf eine Art ritualisiert medialisiertes Handeln zum Zweck der Selbstdarstellung: 
Und da wollen einfach die Leute alle zeigen, dass sie Zeitung gelesen haben und, dass sie das ganz ernst nehmen, was da aktuell wieder Unglaubliches passiert in dem Land. Also zum Teil finde ich es bemühend.

In der Tendenz zeigt sich also, das Parlamentarierinnen und Parlamentarier einschätzen, dass bei Vorstössen und Fragestunden die inhaltliche Dimension zweitrangig ist und mediale Resonanz die erste Präferenz darstellt. Das drängt die Frage auf, was für Ziele Parlamentarierinnen und Parlamentarier mit öffentlicher Präsenz verfolgen. Hier gibt es seitens der Parlamenarierinnen und Parlamentarier, grosso modo, zwei Haupteinschätzungen. Zum einen meinen Parlamentarierinnen und Parlamentarier, öffentliche Präsenz sei Mittel zum Zweck, um über politische Inhalte zu kommunizieren und politische Debatten in der Gesellschaft auszulösen. Zum anderen aber schätzen die Parlamentarierinnen und Parlamentarier aber auch ein, dass der Wunsch nach öffentlicher Präsenz als individueller Profilierung Selbstzweck ist, und zwar, weil öffentliche Präsenz mit höheren Chancen bei den nächsten Wahlen gleichgesetzt wird.

Die Perspektive von öffentlicher Präsenz als Mittel zum Zweck beschreibt ein Mitglied des Nationalrates wie folgt:

Also es geht ja eigentlich darum: Es muss eine Diskussion darüber stattfinden. Und da ist halt, die Medien sind da halt ein ideales Portiermittel. Weil sonst, es werden ja so viele Vorstösse eingereicht und es läuft so viel. Wenn niemand darüber liest, dann weiss auch niemand, was gelau..., also was gemacht wurde. Und deshalb ist es eine sehr sinnvolle Zusammenarbeit.

In einem solchen Verständnis sind Medien ein «Portiermittel» und üben die Funktion aus, politische Inhalte öffentlich zu präsentieren. Ähnlich pragmatisch schätzt ein weiteres Mitglied des Nationalrates diese Übermittlungsfunktion der Medien ein:

Man will ja als Politiker gehört werden, dass die Sachen sollen nach Aussen transportiert werden, die Botschaften sofern man welche hat - oder? - sollen nach Aussen transportiert werden.

Ein anderes Mitglied des Nationalrates beschreibt dieselbe Intention und betont noch die persuasive Dimension:

Die bewusste Kontaktaufnahme mit Medienleuten seitens der Politiker soll dazu führen, dass in der Gesellschaft Goodwill für irgendwelche Ideen geschaffen wird. 
Das Schaffen von «Goodwill» in der Gesellschaft für Ideen im Sinne persuasiver Kommunikation muss dabei keine negative Konnotation haben. Für Ideen mit Argumenten einstehen kann nämlich auch bedeuten, dass ein Diskurs stattfindet, also eine vernunftgeleitete Debatte. Diese persuasive Dimension beschreibt ein weiteres Mitglied als Nationalrates als die Natur von Politik an und für sich:

Aber, schlussendlich besteht Politik darin, seine eigenen Gedanken so rüberzubringen, dass sie am Schluss zu einer Mehrheit werden und man so kann politische Gestaltung ausüben. Und ja, das ist noch zentral. Plus minus machen das alle da drin, sonst wären sie nicht in der Politik.

Ein weiteres Mitglied des Nationalrates kommt ebenfalls zum Schluss, dass öffentliche Kommunikation zum Kern von Politik gehört, und, dass es darum unabdingbar ist, über Medien die Gesellschaft zu erreichen:

Mit der Medienpräsenz kommen sie einfach besser zum Durchbruch. Sie wollen ja in der Politik - und das traue ich, nehme ich einmal an, traue ich allen zu - sie wollen etwas in der Politik beeinflussen. Sie wollen etwas verändern. Ich sage jetzt einmal, sie wollen es verbessern - oder? Nicht? Die meisten meinen es jedenfalls. Und jetzt, der Journalist ist natürlich eine gute Möglichkeit, dass das eben hinausgeht. Und wir haben ja praktisch keine Andere. Also, es hat einmal einer gesagt: Ein Plakat, das nicht beachtet wird oder das kein Aufsehen erregt, hat seinen Beruf verfehlt. Das kann man auch, ein Politiker der keine Botschaft durchbringt, der nichts verändert, der in Vergessenheit gerät, auch wenn er noch so fleissig ist, der hat seinen Beruf verfehlt. Und da helfen die Medien eben schon markant mit, dass einer quasi, dass man jemandem einen Steigbügel, eine Plattform bietet - und das braucht er, das braucht er.

Diese Funktion öffentlicher Präsenz zum Zwecke der gesellschaftlichen Debatte findet auf der inviduellen Ebene statt, aber auch, wie ein Mitglied des Ständerates beschreibt, auf parteipolitischer Ebene:

Ja, also man will natürlich, insbesondere gerade die politischen Parteien, die machen dann ja auch Vorstösse oder man gibt, man steckt sie jemandem, das kann dann ein Fraktionsmitglied sein oder so. Und da will man natürlich dann schon evtl. einen Stein oder respektive eine Lawine lostreten. 
Ein Mitglied des Nationalrates macht eine wichtige Bemerkung. Es ist möglich, Inhalte, Themen und Ideen zu öffentlicher Präsenz zu verhelfen, ohne, dass die eigene Person dabei zwingend zu öffentlicher Präsenz kommt:

Es gehen alle ein bisschen anders mit den Journalisten um. Die einen versuchen, ihre eigenen Geschichten möglichst präsent zu halten und machen sich nicht so stark Überlegungen: Was könnte das Bedürfnis des Journalisten sein? Und andere versuchen, irgendwelche Geschichten zu prägen, ohne dass sie überhaupt vorkommen. Also, weil sie die Sache irgendwie, der Sache dienen wollen.

Diese Bemerkung führt zu der zweiten Art der Ziele, welche Parlamentarierinnen und Parlamentarier mit Medienpräsenz verfolgen, der öffentlichen Präsenz als Selbstzweck. Ein Mitglied des Nationalrates konstatiert in dieser Hinsicht, dass die Bundesversammlung aus Personen besteht, welche allesamt ein gewisses Sendungsbewusstsein haben:

[I]ch habe das Gefühl, wir haben alle recht Freude, uns als Person auch zu zeigen, weil, wir sind da eine Auswahl von 246 Leuten die ein gewisses Sendungsbewusstsein haben, sonst ist man Fehl am Platz.

Dieses Sendungsbewusstsein ist aber, so dasselbe Mitlied des Nationalrates, unterschiedlich ausgesprägt. In einigen Fällen geht es soweit, dass das Streben nach öffentlicher Präsenz Vorrang vor der politischen Arbeit hat:

Es gibt Leute, wo ich ganz genau weiss, die alles machen, das medien-, das öffentlichkeitswirksam ist und nichts machen, wenn man es nicht sieht. Also wir haben Leute in der Kommission, zum Teil, also weniger, aber einzelne, die Zero machen in der Kommission, absolut Null.

Ein weiteres Mitglied des Ständerates beschreibt auch, dass die Suche nach öffentlicher Präsenz nicht bei allen Parlamentarierinnen und Parlamentariern in gleichem Masse ausgeprägt ist:

Das passiert übrigens viel, oder, dass, es gibt solche Politiker, die funktionieren vor allem so, oder. Also ein bisschen böse ausgedrückt: Die funktionieren nach Präsenz in den Medien. Die suchen immer diese dauernde Präsenz. Und es gibt schon solche, die das immer wieder auch so machen. Es gibt andere, die sind sehr fokussiert auf ihre Themen, die wollen einfach, dass ihre Themen weiterverfolgt werden. 
Diese Unterschiede zwischen öffentlicher Präsenz und der parlamentsinternen Arbeit geht so weit, dass das öffentliche, durch Medien erstellte Bild des Parlamentes ein verzerrtest ist, meint ein Mitglied des Nationalrates:

Nicht nur, was sie erreichen wollen, sondern sie erreichen es auch. Man nimmt sie als Schwergewichte wahr und sagt, das sei ein Schwergewicht. Eine meiner erstaunlichsten Feststellungen war, dass es eine Innen- und eine Aussenansicht gibt.

In der Aussenansicht werden jene Parlamentarierinnen und Parlamentarier als politisch arbeitsam wahrgenommen, welche qua ihrer Medienpräsenz öffentlich wahrnehmbar sind. Diese Medienpräsenz hat aber, so die Meinungen der Parlamentarierinnen und Parlamentarier, nicht unbedingt viel mit der parlamentsinternen Arbeit gemein. Den Zweck dieser Medienpräsenz erachten Parlamentarierinnen und Parlamentarier einerseits in dem beschriebenen Sendungsbewusstsein, andererseits aber auch in der Vermutung, dass mehr Medienpräsenz mit höheren Chancen für die Wiederwahl einhergeht. Ein Mitglied des Nationalrates beschreibt diesen Umstand in leicht resigniertem Duktus:

Aber es gibt sicher, man stellt das ja fest, Politiker, die vor den Wahlen extrem präsent sind, und die Bürger sind dann auch, sagen dann auch, jetzt hab ich drei Jahre nichts gehört, oder, und jetzt, na ja.

Ein anderes Mitglied des Nationalrates beobachet ebenfalls ein solches «mediales Erwachen» vor den Wahlen, betrachtet dieses aber aus wohlwollenderer Perspektive:

Also es werden, das kann man wahrscheinlich wissenschaftlich auch feststellen, dass die Vorstossflut wahrscheinlich auf die Wahlen hin zunimmt. Also Wahlkampf hat natürlich mit politischer Tätigkeit zu tun. Also man will ja seinen Wählerinnen und Wählern auch zeigen, dass man politisch aktiv ist.

Der Tenor unter den interviewten Parlamentarierinnen und Parlamentariern bezüglich dieses medialen Erwachens vor den Wahlen ist aber eher ein negativer. Ein Mitglied des Nationalrates wählt entsprechend direkte Worte, um diese Situation zu beschreiben:

Also das ist schon so, dass im Allgemeinen auf die Wahl, auf die Wahlen hin wird der durchschnittliche Politiker, auch wenn er relativ faul ist, plötzlich aktiv und braucht wiederum die Medien. 
Die Motivstrukturen der Parlamentarierinnen und Parlamentarier hinsichtlich ihres medialisierten Handelns besteht also aus unterschiedlichen Dimensionen für unterschiedliche Aspekte medialisierten Handelns. Für die Beeinflussung des Abstimmungsverhaltens zeigt sich, dass diese Form medialisierten Handelns durch zwei Mechanismen bedingt ist. Erstens empfinden Parlamentarierinnen und Parlamentarier einen Druck seitens der Medien, bei Abstimmungen auf eine bestimmte Art und Weise abzustimmen. Am deutlichsten ausgeprägt ist dieser Druck in Phasen von Medienhypes, bei denen Medien ein emotional aufgeladenes Thema in Nachrichtenwellen problematisieren ${ }^{39}$ und damit einen starken Reaktionsdruck auf die Parlamentarierinnen und Parlamentarier ausüben. Ein solches Handeln infolge eines wahrgenommenen Druckes seitens der Medien - ob es einen wie auch immer gearteten Druck tatsächlich gibt, ist irrelevant - bedeutet ein klares Handeln ausschliesslich nach Medienlogiken. Der zweite Mechanismus der Beeinflussung des Abstimmungsverhaltens ist ein Meinungsbildungsprozess der Parlamentarierinnen und Parlamentarier infolge der Wahrnehmung themenbezogener Berichterstattung. Diese Form medialisierten Handelns bei dem Abstimmungsverhalten stellt ein Handeln zugunsten der Politiklogiken dar: Wenn Parlamentarierinnen und Parlamentarier den Auftrag haben, zugunsten der Präferenzen der Wählerschaft zu handeln, dann ist ein Meinungsbildungsprozess unter Einbezug der medialen Berichterstattung prinzipiell durchaus kompatibel mit diesem Auftrag. Ein solcher Meinungsbildungsprozess kann nämlich dazu beitragen, dass Parlamentarierinnen und Parlamentarier zu reflektieren Meinungen gelangen und somit rationaliter zugunsten der Präferenzen der Bürgerinnen und Bürger handeln. Beide Effekte im Kontext des Abstimmungsverhaltens, sowohl reaktive Handeln infolge wahrgenommenen Drucks und das aktive Handeln als Meinungsbildung dürften aber eher schwache Effekte sein, da das medialisierte Abtsimmen grundsätzlich eher punktuell und kontingent ist.

Anders ist die Situation bei den Vorstössen und Fragestunden. Medialisiertes Handeln spielt in diesen zwei Teilarenen der Bundesversammlung, so die klare Einschätzung der Parlamentarierinnen und Parlamentarier, eine wichtige Rolle. Auch schätzen die Parlamentarierinnen und Parlamentarier deutlich ein, dass die sachliche Dimension sekundär ist und, dass stattdessen das Erlangen öffentlicher Resonanz das primäre Ziel medialisierter Vorstösse und medialisierter Fragen in den Fragestunden darstellt.

39 Bisweilen wird die Beobachtung, dass Journalistinnen und Journalisten insbesondere in Phasen von Medienhypes ein Thema konzentriert und auf eine sehr ähnliche Art behandeln, «Rudeljournalismus» (Frank 2003) genannt. Obschon der Begriff Rudeljournalismus recht eindeutig negativ konnotiert ist, ist dieses Konzept handlungstheoretisch insofern nützlich, als es den Vorgang des Medienhypes nicht als konzertierte Aktion unterschiedlicher Medien deutet, sondern als Aggregation individuellen journalistischen Handelns. 
Bei der Frage schliesslich, worin die Parlamentarierinnen und Parlamentarier das allgemeine Ziel von Medienpräsenz sehen, sind zwei wesentliche Deutungen vorhanden. Ein Teil der Parlamentarierinnen und Parlamentarier begründet Medienpräsenz als notwendige Bedingung des Politischen an und für sich: Politik bedeutet in dieser Lesart, nicht nur innerhalb der jeweiligen politischen Institution aktiv zu werden, sondern auch, die politische Arbeit und die eigenen Ideen und Überzeugungen öffentlich kundzutun, um damit die Gesellschaft am Politischen teilhaben zu lassen. Eine solche Auffassung des Ziels von Medienpräsenz bedeutet sehr deutlich medialisiertes Handeln zugunsten der Politiklogiken. Die Parlamentarierinnen und Parlamentarier sehen aber auch eine zweite Komponente von Medienpräsenz, welche die Politiklogiken nicht tangiert: Den individuellen Bedarf nach öffentlicher Wahrnehmbarkeit als Selbstzweck, gekoppelt mit der individuellen Annahme, dass mehr Medienpräsenz die Chancen auf die Wiederwahl erhöht. Diese Einstellung geht bisweilen soweit, dass einzelne Parlamentarierinnen und Parlamentarier stärker auf Medienpräsenz denn auf Arbeit innerhalb des Parlamentes bedacht sind. Das Ziel, wiedergewählt zu werden, ist eine Präferenz, welche weder den Präferenzen der Bürgerinnen und Bürger, noch den Präferenzen der Medien zuzuordnen ist. Die Wiederwahl ist eine individuell gehegte Präferenz, welche also weder die Politiklogiken noch die Medienlogiken tangiert. Wenn das Verhältnis der Wählerinnen und Wähler zu den Parlamentarierinnen und Parlamentariern als Prinzipal-Agent-Beziehung gedacht wird, dann kann die Wiederwahl des Agenten, logisch gesehen, nicht eine Präferenz des Prinzipals sein, da die Wahlentscheidung jenen Entscheidungsmoment darstellt, in welchem der Prinzipal die Arbeit des Agenten evaluiert.

Gesamthaft betrachtet spricht also Einiges dafür, die Hypothese H7 zu verwerfen. Mit der Hypothese H7 postuliere ich, dass Parlamentarierinnen und Parlamentarier nur dann medialisiert handeln, wenn sie damit auch den Politiklogiken Vorschub leisten können. Dies scheint recht deutlich nicht der Fall zu sein, denn sowohl beim Abstimmungsverhalten, als auch bei den Vorstössen und Fragestunden, wie auch bei der Frage des allgemeinen Ziels von Medienpräsenz handeln Parlamentarierinnen und Parlamentarier teilweise zuungunsten der Politiklogiken.

\subsubsection{H8 und H9: Politiklogiken und Medienlogiken in den Medien}

In den Hypothesen H8 und H9 widme ich mich dem Handeln der Journalistinnen und Journalisten. Ich postuliere zunächst in Hypothese H8, dass die Berichterstattung über das Parlament nicht für alle Teilarenen denselben Umfang hat. In Hypothese H9 postuliere ich, dass Journalistinnen und Journalisten sowohl nach Medienlogiken als auch nach Politiklogiken handeln: 
- Hypothese H8: Medien berichten am meisten über die traktandierten Geschäfte, weniger über die eingereichten Vorstösse und am wenigsten über die Fragestunden im Nationalrat.

- Hypothese H9: Journalistinnen und Journalisten handeln in gleichem Umfang nach Medienlogiken wie nach Politiklogiken.

Um zunächst die Hypothese H8 zu prüfen, werte ich die inhaltsanalytischen Daten quantitativ aus. Ähnlich wie bei den Hypothesen H3 und H4 in Unterunterabschnitt 5.1.2 modelliere ich zu diesem Zweck sowohl Zeitreihen wie auch einfache lineare Modelle. Um zu prüfen, welchen Einfluss die regulären Geschäfte im National- und im Ständerat sowie die eingereichten Vorstösse auf die Berichterstattung haben, modelliere ich für jede Session je drei Zeitreihen; eine Zeitreihe pro Sessionswoche. Da an nur zwei Tagen pro Session Fragestunden stattfinden, modelliere ich zu diesen je zwei einfache lineare Modelle pro Session. Sowohl in den Zeitreihen als auch in den einfachen linearen Modellen ist die Medienagenda die Antwortvariable und die parlamentarischen Teilarenen sind die Prädiktoren. In Tabelle 21 sind die Ergebnisse der drei Zeitreihen für 1999 zusammengefasst.

Tabelle 21: Einfluss regulärer Geschäfte aus dem National- und Ständerat sowie der Vorstösse auf die Berichterstattung für 1999.

\begin{tabular}{llrrr}
\hline Zeitraum & Prädiktoren & Schätzung & PCSE & $\mathrm{p}$ \\
\hline \multirow{2}{*}{ 20.09. - 24.09. } & Nationalrat $_{t-1}$ & 0.092 & 0.005 & 0.000 \\
& Ständerat $_{t-1}$ & 0.095 & 0.005 & 0.000 \\
& Vorstösse $_{t-1}$ & -0.002 & 0.007 & 0.807 \\
\hline 27.09. - 01.10. & Nationalrat $_{t-1}$ & 0.106 & 0.006 & 0.000 \\
& Ständerat $_{t-1}$ & 0.051 & 0.007 & 0.000 \\
& Vorstösse $_{t-1}$ & 0.015 & 0.009 & 0.071 \\
\hline \multirow{2}{*}{ 04.10. - 09.10. } & Nationalrat $_{t-1}$ & 0.040 & 0.009 & 0.000 \\
& Ständerat $_{t-1}$ & 0.162 & 0.013 & 0.000 \\
& Vorstösse $_{t-1}$ & 0.001 & 0.011 & 0.912 \\
\hline
\end{tabular}

Das korrigierte $R^{2}$ beträgt für die erste Woche 0.053 , für die zweite Woche 0.091 und für die dritte Woche 0.011 .

Für die Modelle der drei Wochen von 1999 fällt auf, dass der National- und der Ständerat in allen drei Wochen Effekte mit sehr tiefen p-Werten ausüben. Die Stärke der Effekte variiert aber: In der ersten Woche sind beide Effekte ähnlich klein; in der zweiten Woche ist der Effekt des Nationalrates stärker und jener des Ständerates schwächer; in der dritten Woche dreht dieses Verhältnis und der Effekt des Ständerates ist stärker, jener des Nationalrates schwächer. In allen drei Wochen üben die Vorstösse nur einen sehr kleinen Effekt aus, was bedeutet, dass 
kaum systematisch über Vorstösse berichtet wird. Zudem ist der p-Wert für die Vorstösse nur in der zweiten Woche in einem solchen Masse klein, dass die Wahrscheinlichkeit für den Effekt plausibel ist. Die Ergebnisse der Modelle für 2005 sind in Tabelle 22 zusammengefasst.

Tabelle 22: Einfluss regulärer Geschäfte aus dem National- und Ständerat sowie der Vorstösse auf die Berichterstattung für 2005.

\begin{tabular}{llrrr}
\hline Zeitraum & Prädiktoren & Schätzung & PCSE & $\mathrm{p}$ \\
\hline 19.09. - 23.09. & Nationalrat $_{t-1}$ & 0.117 & 0.004 & 0.000 \\
& Ständerat $_{t-1}$ & 0.124 & 0.005 & 0.000 \\
& Vorstösse $_{t-1}$ & -0.002 & 0.007 & 0.714 \\
\hline \multirow{2}{*}{ 6.09. - 30.09. } & Nationalrat $_{t-1}$ & 0.060 & 0.004 & 0.000 \\
& Ständerat $_{t-1}$ & 0.071 & 0.005 & 0.000 \\
& Vorstösse $_{t-1}$ & -0.004 & 0.009 & 0.634 \\
\hline 03.10. - 08.10. & Nationalrat $_{t-1}$ & 0.085 & 0.004 & 0.000 \\
& Ständerat $_{t-1}$ & 0.071 & 0.004 & 0.000 \\
& Vorstösse $_{t-1}$ & 0.019 & 0.014 & 0.182 \\
\hline
\end{tabular}

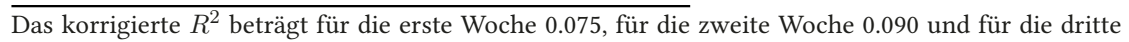
Woche 0.035 .

Die Modelle für 2005 zeichnen für alle drei Wochen ein sehr deutliches Bild: Das Geschehen im National- und im Ständerat fliesst konstant, wenn auch mit kleinen Effekten, in die Berichterstattung ein, gepaart mit sehr tiefen p-Werten. Die Berichterstattung über die Geschäfte des National- und des Ständerates ist in der ersten Woche stärker als in den zwei folgenden Wochen. Vorstösse hingegen sind nur in sehr geringem Umfang Gegenstand der Medien. Da zudem die p-Werte für diese kleinen Effekte in einer Woche hoch, in den restlichen zwei Wochen sehr hoch ist, ist der Effekt der Vorstösse generell sehr unwahrscheinlich. Insgesamt sind die Effekte für die Modelle 2005 den Effekten der Modelle für 1999 sehr ähnlich. Die Ergebnisse der Modelle für 2011 schliesslich sind in Tabelle 23 zusammengefasst.

Das Muster der Ergebnisse der Modelle für 1999 und 2005 ist erneut bei den Ergebnissen der Modelle für 2011 vorhanden. Sowohl der National- als auch der Ständerat üben über alle drei Wochen hinweg einen kleinen, aber konstanten Effekt aus, und zwar bei konstant tiefen pWerten. Die Vorstösse hingegen üben über alle drei Wochen hinweg nur einen vernachlässigbar kleinen Effekt aus, und dies bei sehr hohen p-Werten. Die Modelle für 1999, 2005 und 2011 legen also den Schluss nahe, dass die Medien stetig über das Geschehen im National- und im Ständerat berichten, aber nur vereinzelt und nicht systematisch über die eingereichten Vorstösse. 
Tabelle 23: Einfluss regulärer Geschäfte aus dem National- und Ständerat sowie der Vorstösse auf die Berichterstattung für 2011.

\begin{tabular}{llrrr}
\hline Zeitraum & Prädiktoren & Schätzung & PCSE & $\mathrm{p}$ \\
\hline 12.09. - 16.09. & Nationalrat $_{t-1}$ & 0.156 & 0.004 & 0.000 \\
& Ständerat $_{t-1}$ & 0.062 & 0.005 & 0.000 \\
& Vorstösse $_{t-1}$ & 0.006 & 0.005 & 0.285 \\
\hline \multirow{2}{*}{ 19.09. - 23.09. } & Nationalrat $_{t-1}$ & 0.087 & 0.006 & 0.000 \\
& Ständerat $_{t-1}$ & 0.066 & 0.006 & 0.000 \\
& Vorstösse $_{t-1}$ & -0.001 & 0.004 & 0.773 \\
\hline \multirow{2}{*}{ 6.09. - 01.10. } & Nationalrat $_{t-1}$ & 0.048 & 0.004 & 0.000 \\
& Ständerat $_{t-1}$ & 0.080 & 0.006 & 0.000 \\
& Vorstösse $_{t-1}$ & -0.007 & 0.012 & 0.551 \\
\hline
\end{tabular}

Das korrigierte $R^{2}$ beträgt für die erste Woche 0.107 , für die zweite Woche 0.047 und für die dritte Woche 0.015 .

Tabelle 24: Einfluss der Fragestunden auf die Berichterstattung für 1999, 2005 und 2011.

\begin{tabular}{llrrr}
\hline Jahr & Prädiktor & Schätzung & SE & $\mathrm{p}$ \\
\hline \multirow{2}{1999}{} & Fragestunde 1 & 0.015 & 0.022 & 0.495 \\
& Fragestunde 2 & 0.833 & 0.032 & 0.000 \\
\hline \multirow{2}{*}{2005} & Fragestunde 1 & 0.045 & 0.031 & 0.146 \\
& Fragestunde 2 & 0.047 & 0.030 & 0.119 \\
\hline \multirow{2}{2}{2011} & Fragestunde 1 & 0.099 & 0.038 & 0.010 \\
& Fragestunde 2 & -0.006 & 0.029 & 0.839 \\
\hline
\end{tabular}

Das korrigierte $R^{2}$ beträgt für das Modell zur ersten Fragestunde $1999-0.001$, für das zur zweiten Fragestunde 0.409; für 2005 beträgt das korrigierte $R^{2}$ je 0.001 und 0.119 ; für 2011 je 0.005 und -0.001 .

Der Einfluss der Fragestunden auf die Berichterstattung für 1999, 2005 und 2011 ist in Tabelle 24 zusammengefasst. Die einfachen linearen Modelle, welche abbilden, wie stark die Fragestunden die Berichterstattung am folgenden Tag beeinflussen, deuten darauf hin, dass die Medien meistens nicht systematisch über die Fragestunden berichten. In den Modellen sind aber zwei Ausnahmen vorhanden. In dem Modell für die erste Fragestunde 2011 ist ein Effekt zu beobachten, der zwar gering, aber nicht komplett irrelevant ist, zumal er einen relativ tiefen p-Wert aufweist. Die zweite Ausnahme ist der Effekt der zweiten Fragestunde 1999: Hier ist nicht nur der p-Wert sehr gering, sondern auch der Effekt sehr stark. Es handelt sich dabei nicht um eine statistische Kuriosität, sondern um eine Eigenheit des politischen Diskurses im Herbst 1999. Damals gab es politische Turbulenzen rund um die Landessausstellung «Expo.01» (Welche im Herbst 1999 um ein Jahr verschoben und zur «Expo.02» umbenannt wurde), und 
die Landesausstellung wurde in dieser einen Fragestunde intensiv besprochen, was wiederum von den Medien aufgegriffen wurde.

Insgesamt findet die Hypothese H8 nur teilweise Unterstützung durch die Daten. Einerseits ist recht deutlich, dass die Medien am intensivsten über die Geschäfte des National- und des Ständerates berichten. Weniger klar ist aber, ob die Berichterstattung über Vorstösse grösser ist als über Fragestunden; die Daten sprechen eher dagegen.

Die Hypothese H9 postuliert, dass Journalistinnen und Journalisten in gleichem Masse nach Medienlogiken wie nach Politiklogiken handeln. Diese Hypothese prüfe ich sowohl anhand der inhaltsanalytischen Daten wie auch anhand der Interviews. Um die Hypothese H9 auf Ebene der Inhaltsanalyse zu prüfen, trenne ich die Berichterstattung in zwei Teile, die Parlamentsberichterstattung und die sonstige Politikberichterstattung ${ }^{40}$. Ich modelliere mit diesen zwei getrennten Medienagenden Zeitreihen, bei denen die Parlamentsberichterstattung die Antwortvariable bildet. Die Prädiktoren sind die Geschäfte des National- und des Ständerates, die eingereichten Vorstösse sowie jener Teil der Medienagenda, welcher keine Parlamentsberichterstattung enthält. Dieses Modell zeigt auf, ob die Parlamentsberichterstattung stärker durch die Inhalte des Parlamentes beeinflusst wird, oder durch die sonstigen Inhalte der Medien. Wenn Journalistinnen und Journalisten sich in der Parlamentsberichterstattung ausschliesslich nach Medienlogiken richten, dann ist anzunehmen, dass die Parlamentsberichterstattung inhaltlich eher die sonstige Agenda der Medien abbildet als die parlamentarische Agenda. Um diese Modelle prüfen zu können ist es, logischerweise, nötig, nicht mit den konkreten Themen, sondern mit Politikfeldern zu modellieren. Die konkreten Themen der Parlaments- und der restlichen Politikberichterstattung unterscheiden sich natürlich, sodass hier keine Beeinflussung möglich ist. Es ist aber denkbar, dass die Schwerpunktsetzung bei den Politikfeldern für die Parlamentsberichterstattung und für die sonstige Politikberichterstattung ähnlich ausfällt, wenn Medienlogiken tatsächlich dominant sind. Die Ergebnisse der Modelle für 1999 sind in Tabelle 25 zusammengefasst.

Die Ergebnisse der Modelle für 1999 sind für alle drei Wochen fast identisch. In allen Wochen übt die Zusammensetzung der Politikfelder des National- und des Ständerates einen substanziellen Effekt aus, gepaart mit stets tiefen bis sehr tiefen p-Werten. Die Zusammensetzung der Politikfelder in der Berichterstattung ohne Parlamentsbezug übt nur in der ersten Woche einen grösseren Effekt aus, aber der Effekt ist ein negativer, was bedeutet, dass die Zusammensetzung der Politikfelder der Nicht-Parlamentsberichterstattung die Zusammensetzung der Politikfel-

\footnotetext{
40 $\quad$ Bei der qualitativen Inhaltsanalyse wurde auch dieses Merkmal bei den Untersuchungseinheiten in der Medienstichprobe mitcodiert.
} 
Tabelle 25: Einfluss der Medienberichterstattung ohne Parlamentsbezug, der regulären Geschäfte aus dem National- und Ständerat sowie der Vorstösse auf die Parlamentsberichterstattung für 1999.

\begin{tabular}{llrrr}
\hline Zeitraum & Prädiktoren & Schätzung & PCSE & $\mathrm{p}$ \\
\hline 20.09. - 24.09. & ${\text { Medien ohne } \mathrm{PB}_{t-1}}$ & -0.200 & 0.135 & 0.145 \\
& Nationalrat $_{t-1}$ & 0.348 & 0.049 & 0.000 \\
& Ständerat $_{t-1}$ & 0.392 & 0.043 & 0.000 \\
& Vorstösse $_{t-1}$ & 0.132 & 0.089 & 0.145 \\
\hline 27.09. - 01.10. & ${\text { Medien ohne } \mathrm{PB}_{t-1}}$ & 0.071 & 0.130 & 0.585 \\
& Nationalrat $_{t-1}$ & 0.365 & 0.058 & 0.000 \\
& Ständerat $_{t-1}$ & 0.129 & 0.053 & 0.018 \\
& Vorstösse $_{t-1}$ & -0.085 & 0.067 & 0.205 \\
\hline 04.10. - 09.10. & ${\text { Medien ohne } \mathrm{PB}_{t-1}}$ & 0.007 & 0.095 & 0.946 \\
& Nationalrat $_{t-1}$ & 0.350 & 0.065 & 0.000 \\
& Ständerat $_{t-1}$ & 0.249 & 0.076 & 0.002 \\
& Vorstösse $_{t-1}$ & -0.020 & 0.093 & 0.830 \\
\hline
\end{tabular}

Die Berechnungen wurden nicht anhand konkreter einzelner Themen, sondern anhand der Politikfelder der Themen gemacht, weil Parlamentsberichterstattung und Nicht-Parlamentsberichterstattung logischerweise unterschiedliche konkrete Themen beinhalten. «Medien ohne $\mathrm{PB}$ » ist abgekürzt für «Medien ohne Parlamentsberichterstattung». Das korrigierte $R^{2}$ beträgt für die erste Woche 0.594 , für die zweite Woche 0.458 und für die dritte Woche 0.304 .

der der Parlamentsberichterstattung nicht beeinflusst. Zudem weist dieser Effekt einen eher hohen p-Wert auf, sodass nicht sehr Wahrscheinlich ist, dass der Effekt tatsächlich besteht. Die Ergebnisse der Modelle für 2005 sind in Tabelle 26 zusammengefasst.

Die Ergebnisse für die Modelle der drei Wochen der Session 2005 sind weniger deutlich als in den Modellen für 1999, sie zeigen aber in die gleiche Richtung: Die Zusammensetzung der Politikfelder der Parlamentsberichterstattung ist in den ersten zwei Wochen stark durch den National- und den Ständerat beeinflusst, in der dritten Woche nur durch den Ständerat. Die Effekte der Politikberichterstattung ohne Bezug zum Parlament sind für die zweite und dritte Woche angesichts der hohen p-Werte eher unwahrscheinlich. In der ersten Woche aber ist der p-Wert tief genug, damit der Effekt als möglicherweise relevant gelten kann. Allerdings ist der Effekt in der ersten Woche im Vergleich zum Effekt der Geschäfte des National- und des Ständerates um knapp ein Drittel schwächer, und er weist darüber hinaus einen deutlich höheren p-Wert aus. Die Ergebnisse der Modelle für 2011 schliesslich sind in Tabelle 27 zusammengefasst.

Die Modelle für die ersten zwei Wochen der Session 2011 zeichnen erneut dasselbe Bild wie die Modelle für 1999 und 2005: Die grösseren Effekte mit tieferem p-Wert gehen von der Zusammensetzung der Politikfelder der Geschäfte des National- und des Ständerates aus. Auch in der dritten Woche haben die Geschäfte des National- und des Ständerates subsanzielle Effekte, 
Tabelle 26: Einfluss der Medienberichterstattung ohne Parlamentsbezug, der regulären Geschäfte aus dem National- und Ständerat sowie der Vorstösse auf die Parlamentsberichterstattung für 2005.

\begin{tabular}{|c|c|c|c|c|}
\hline Zeitraum & Prädiktoren & Schätzung & PCSE & $\mathrm{p}$ \\
\hline \multirow{4}{*}{ 19.09. - 23.09. } & Medien ohne $\mathrm{PB}_{t-1}$ & 0.207 & 0.107 & 0.060 \\
\hline & Nationalrat $_{t-1}$ & 0.313 & 0.032 & 0.000 \\
\hline & Ständerat $_{t-1}$ & 0.320 & 0.044 & 0.000 \\
\hline & Vorstösse $_{t-1}$ & -0.067 & 0.058 & 0.257 \\
\hline \multirow[t]{4}{*}{ 26.09. - 30.09. } & Medien ohne $\mathrm{PB}_{t-1}$ & 0.084 & 0.081 & 0.303 \\
\hline & Nationalrat $_{t-1}$ & 0.366 & 0.027 & 0.000 \\
\hline & Ständerat $_{t-1}$ & 0.286 & 0.033 & 0.000 \\
\hline & Vorstösse $_{t-1}$ & -0.063 & 0.059 & 0.290 \\
\hline \multirow[t]{4}{*}{ 03.10. - 08.10} & Medien ohne $\mathrm{PB}_{t-1}$ & 0.113 & 0.135 & 0.406 \\
\hline & Nationalrat $_{t-1}$ & 0.470 & 0.058 & 0.000 \\
\hline & Ständerat $_{t-1}$ & 0.050 & 0.048 & 0.297 \\
\hline & Vorstösse $_{t-1}$ & 0.048 & 0.107 & 0.657 \\
\hline
\end{tabular}

Die Berechnungen wurden nicht anhand konkreter einzelner Themen, sondern anhand der Politikfelder der Themen gemacht, weil Parlamentsberichterstattung und Nicht-Parlamentsberichterstattung logischerweise unterschiedliche konkrete Themen beinhalten. «Medien ohne PB» ist abgekürzt für «Medien ohne Parlamentsberichterstattung». Das korrigierte $R^{2}$ beträgt für die erste Woche 0.643 , für die zweite Woche 0.737 und für die dritte Woche 0.535 .

aber in dieser Woche geht der stärkste Effekt von der Zusammensetzung der Politikfelder der Nicht-Parlamentsberichterstattung der Medien aus. Über alle Modelle hinweg hat die NichtParlamentsberichterstattung nur in einer Woche einen wahrscheinlichen Effekt, die Geschäftes des Nationalrates hingegen in neun, die Geschäfte des Ständerates in acht modellierten Wochen einen wahrscheinlichen Effekt. Die quantitativen Daten sprechen also eher gegen die Hypothese H9, da sich Journalistinnen und Journalisten bei der Parlamentsberichterstattung deutlich stärker am Parlamentsgeschehen denn an den eigenen narrativen Präferenzen orientieren.

Auf der Ebene der qualitativen Interviews kommen die journalistischen Motivstrukturen in zwei Dimensionen zum Ausdruck. Einerseits bei der Frage, nach welchen Kriterien Journalistinnen und Journalisten auswählen, über welche Inhalte der Session sie berichten. Andererseits auch bei der allgemeineren Frage, was für Ziele die Journalistinnen und Journalisten mit ihrer Parlamentsberichterstattung verfolgen. Hinsichtlich der Auswahlkriterien für die Parlamentsberichterstattung ist das häufigste Argument die gesellschaftliche Relevanz der Themen. So beschreibt etwa eine Journalistin:

Ja, also gesellschaftliche Relevanz ist bei uns schon auch sehr wichtig. Also, ja klar, also gesellschaftliche Relevanz ist das Thema Nummer 1 bei uns. Also, ja, wir sind 
Tabelle 27: Einfluss der Medienberichterstattung ohne Parlamentsbezug, der regulären Geschäfte aus dem National- und Ständerat sowie der Vorstösse auf die Parlamentsberichterstattung für 2011.

\begin{tabular}{llrrr}
\hline Zeitraum & Prädiktoren & Schätzung & PCSE & $\mathrm{p}$ \\
\hline 12.09. - 16.09. & ${\text { Medien ohne } \mathrm{PB}_{t-1}}$ & 0.041 & 0.104 & 0.696 \\
& Nationalrat $_{t-1}$ & 0.307 & 0.040 & 0.000 \\
& Ständerat $_{t-1}$ & 0.152 & 0.046 & 0.002 \\
& Vorstösse $_{t-1}$ & -0.042 & 0.067 & 0.529 \\
\hline 19.09. - 23.09. & ${\text { Medien ohne } \mathrm{PB}_{t-1}}$ & 0.111 & 0.083 & 0.184 \\
& Nationalrat $_{t-1}$ & 0.369 & 0.052 & 0.000 \\
& Ständerat $_{t-1}$ & 0.466 & 0.049 & 0.000 \\
& Vorstösse $_{t-1}$ & -0.056 & 0.045 & 0.220 \\
\hline \multirow{2}{*}{ 6.09. - 01.10. } & ${\text { Medien ohne } \mathrm{PB}_{t-1}}$ & 0.418 & 0.127 & 0.002 \\
& Nationalrat $_{t-1}$ & 0.142 & 0.052 & 0.008 \\
& Ständerat $_{t-1}$ & 0.202 & 0.043 & 0.000 \\
& Vorstösse $_{t-1}$ & -0.037 & 0.122 & 0.762 \\
\hline
\end{tabular}

Die Berechnungen wurden nicht anhand konkreter einzelner Themen, sondern anhand der Politikfelder der Themen gemacht, weil Parlamentsberichterstattung und Nicht-Parlamentsberichterstattung logischerweise unterschiedliche konkrete Themen beinhalten. «Medien ohne PB» ist abgekürzt für «Medien ohne Parlamentsberichterstattung». Das korrigierte $R^{2}$ beträgt für die erste Woche 0.449 , für die zweite Woche 0.732 und für die dritte Woche 0.363 .

ja auch die Bundeshausredaktion, also wir sind, wir können uns diese Seriosität noch am ehesten leisten, sage ich jetzt Mal, ja.

Die Relevanz der Themen bemisst sich dabei letztlich an einem quantifizierbaren Kriterium, nämlich der Anzahl Menschen, welche von diesem politischen Thema betroffen sind. So erklärt eine Journalistin:

Es geht ja meistens um eine Veränderung, die viele Leute betrifft zum Beispiel. Also wie, dass es viele Leute betrifft, dass es viele Leute ärmer macht oder reicher macht. Oder zum Beispiel, so Bildungsthemen sind grundsätzlich sehr relevant, weil alle Mal in die Schule gingen und viele haben Kinder, die in die Schule gehen usw. Steuern zum Beispiel ist ein schwieriges Thema, aber wenn du es so herunter brechen kannst, dass du dem Leser sagen kannst, warum es ihn betrifft und warum es für ihn wichtig ist, ist es ein sehr wichtiges Thema - so einfach, ja. Ja, [...] und die Betroffenheit der Leute ist wichtig, aber nicht nur emotionale Betroffenheit, sondern auch einfach sachlich, in einem sachlichen Sinn.

Nebst der Relevanz ist auch der Neuigkeitswert eines parlamentarischen Inhaltes als Selektionskriterium von Bedeutung, wie eine weitere Journalistin festhält: 
Und ja es ist; die meisten Gesetzesvorlagen gehen ja dreimal durch die Räte und dann kommt es immer ein bisschen darauf an: Wenn etwas zum ersten mal kommt, schauen wir das an.

Als relevant erachtete Themen können aber auch eine hohe Komplexität haben; mit der Relevanz politischer Vorlagen im Sinne ihrer Tragweite steigt in der Regel auch ihre Komplexität. Um diesen Umstand wissen auch die Journalistinnen und Journalisten und sie versuchen aktiv, komplexe Themen nicht zu vermeiden, sondern sie angesichts ihrer Relevanz doch zu thematisieren. Ein Journalist meint dazu:

Es ist halt so, dass sehr, sehr technische Sachen ja enorm relevant sein können. Die sind einfach wahnsinnig schwierig, in einer Zeitung, oder via Medien generell zu vermitteln.[...] Wenn Sie so, vielleicht müssen Sie mit Schlagworten noch das Ganze vereinfachen, und dann interessiert es nämlich plötzlich, weil die Leute, ja, sie müssen wie betroffen sein, also wirklich.

Gemäss dieser Schilderung wird aktiv versucht, komplexe Themen über komplexitätsreduzierende Heuristiken zu verarbeiten. Ein ähnliches Vorgehen beschreibt ein weiterer Journalist:

Also weil man kann eben auch komplexe Themen, finde ich, kann man journalistisch coole Sachen machen, wenn man sich wirklich mit dem befasst und das eigentlich verständlich erklären kann. Eben, Energiewende, oder, das ist für mich immer so ein Paradebeispiel von technischen Fragen. Oder eben, gerade in der Verkehrspolitik, oder, Strassenfonds und Benzinpreiserhöhung und so. Das ist ja auch nicht, das ist vordergründig sehr einfach zu verstehen, aber man kann dort trotzdem relativ viel rausholen, indem man eigentlich die komplexen Informationen, die man von der Verwaltung erhält, herunterbricht.

Die Präferenz für als gesellschaftlich relevant erachtete Themen ist ein Handeln zugunsten der Politiklogiken. Natürlich wäre im Einzelfall zu prüfen, welche konkreten Themen aus welchen Gründen als relevant erachtet werden, und, od diese Einschätzung irgendwie objektivierbar ist. Handlungstheoretisch aber ist das mit diesem Relevanzkriterium verfolgte Ziel eines, welches prinzipiell kompatibel mit Politiklogiken ist. Der Medialisierungsschritt in Form der Komplexitätsreduktion ist in diesem Sinne ein Schritt, der den Politiklogiken nicht abträglich ist. Das Kriterium der Relevanz fliesst auch in die Auswahl der Vorstösse, über welche berichtet wird. Ein Journalist erklärt, dass sich die Relevanz eines Vorstosses auch daran bemisst, wie gross die Erfolgsschancen des Vorstosses sind: 
Also wenn einfach ein, irgendein Hinterbänkler da eine Idee lanciert, wo wir genau wissen, das ist chancenlos, dann machen wir vermutlich auch nichts.

Obschon also die gesellschaftliche Relevanz von allen Journalistinnen und Journalisten als wichtiges Kriterium beschrieben wird, ist Relevanz nicht das einzige und vielleicht nicht das dominierende Auswahlkriterium. Ein Journalist beschreibt etwa, dass der Grad der Kontroverse der parlamentarischen Inahlte ein Kriterium darstellt:

Und dann gibts solche [Themen], die eben kontrovers sind. Die politisch kontrovers sind. Wir nehmen natürlich schon die, bei denen wir davon ausgehen können, dass es im Plenum eine engagierte, lebhafte, ja, eben auch kontroverse Diskussion geben wird.

Diese Orientierung an Kontroversen und Konflikten stellt bei den meisten Journalistinnen und Journalisten ein bedeutendes Kriterium dar. Eine Journalistin meint dazu kurz und knapp:

Wenn etwas sehr konfliktträchtig ist, selbstverständlich betreuen wir das.

Ein weiteres für alle Journalistinnen und Journalisten wichtiges Kriterium, welches nicht unbedingt zugunsten der Politiklogiken ist, ist die Exklusivität der Inhalte. Ein Journalist erklärt, dass eingereichte Vorstösse nicht sehr interessant für ihn bzw. das Medium seien, weil eingereichte Vorstösse für alle öffentlich zugänglich sind:

Also wenn es, also gut, ein exklusiver, toller Vorstoss ist gut, aber in dem Sinne Ratsgeschäfte und Vorstösse eingereichte sind für uns natürlich weniger gut, als wenn wir irgendetwas Neues haben, das sonst niemand hat. Das heisst, das kann irgendwie sein: Kehrtwende in der CVP. Oder irgend so was in die Richtung, oder, das in dem Sinne relevant ist und so. Und das erfährt man halt wirklich häufig einfach in der Wandelhalle, wenn man einfach dort ist und mit den Leuten spricht und so.

Über Vorstösse wird punktuell aber dennoch berichtet, denn über bilateralen Austausch mit Parlamentarierinnen und Parlamentariern erhalten Journaistinnen und Journalisten exklusiven Zugang zu Vorstössen, noch bevor diese eingereicht sind. Ein Journalist meint dazu:

Also es gibt, wenn, also es gibt Zeiten, da kann ich wirklich fast jedes Mal, wenn ich da in die Wandelhalle rübergehe, habe ich nachher wieder einen oder zwei Vorstösse in der Tasche, die mir jemand zugesteckt hat. 
Die Exklusivität der Inhalte sieht ein Journalist denn auch als eines seiner generellen Ziele bei Parlamentsberichterstattung:

Und das heisst, dass man in dem Sinne versucht, Dinge herauszufinden und eben, wenn möglich, halt als Erster, oder, die ich in dem Sinne für meine Leser interessant finde, die ihnen je nach dem, weiss auch nicht, eine Erklärung sein kann, wie die Schweiz funktioniert.

Das Ziel des zitierten Journalisten besteht allerdings nicht in blosser Exklusivität, sondern in Exklusivität gepaart mit der vorher beschriebenen gesellschaftlichen Relevanz. Als übergeordnetes Ziel ihrer Arbeit haben einige Journalistinnen und Journalisten beschrieben, dass sie dazu beitragen möchten, dass Medien eine Kontroll- und Kritikfunktion ausüben. Eine Journalistin beschreibt die Funktion des Kontrollorgans als Ideal, nach welchem man sich richte:

Ich denke, wir sehen uns - also da kann ich glaube ich auch für unser Team [sprechen] - , wir sehen uns eben schon auch Kontrollorgan. Also das ist ein Idealbild natürlich. Ist nicht so, dass uns das immer gelingt.

Diese Kontrollfunktion beschreibt eine weitere Journalistin als das Hinterfragen des Parlamentsgeschehens aus einer breiteren, kontextualisierenden Sicht:

wir hinterfragen ja in erster Linie auch. Also natürlich, wir sprechen jetzt immer nur über die Sessionen und dort ist der Berichterstattungscharakter relativ hoch. Aber wir versuchen ja auch die Arbeit des Parlaments und auch die Entscheide, die ja manchmal widersprüchlich sein können, von einem Jahr auf das andere zum Beispiel.

Eine andere Journalistin betont ebenfalls das Ziel der Einordnungsfunktion:

Eine neutrale Berichterstattung, auch eine Information, die Menschen quasi darüber zu informieren, was aktuell geht und wie die verschiedenen Meinungen dazu lauten, aber auch, wie das Ganze im Gesamtkontext zu sehen ist, also auch diese einordnende Funktion, die wir haben, die auch immer wichtiger ist. Also, wie ist das im Gesamtkontext zu sehen, wie sind die Zusammenhänge mit anderen Themen, mit anderen Ländern, also das auch aufzuzeigen.

Dieses journalistische Einordnen und Erklären beschreibt eine Journalistin als Aufklärungsfunktion: 
Ich möchte, ja schon, für mich ist Journalismus schon auch Aufklärung, also im klassischen Sinne eigentlich. Dass ich zeigen möchte, was Wichtiges passiert und den Leuten auch ein bisschen erklären vielleicht, was Wichtiges passiert.

Ein Journalist relativiert diese an den Politiklogiken orientierten Ziele ein Stück weit mit dem Verweis, dass im journalistischen Alltag auch eine wichtige Rolle spielt, wie der journalistische Output rezipiert wird:

Wenn [Zeitungstitel] am Tag 1 merkt, mit diesem Thema auf der Titelseite verkaufen wir die Zeitung gut, dann versucht er das gleiche Thema am nächsten Tag zwingend noch einmal auf die Titelseite zu hebeln, sag ich Mal, und sucht dann entsprechende Aufhänger. Also das ist ein Faktor sicher.

Die Ergebnisse der qualitativen Interviews deuten an, dass Journalistinnen und Journalisten in ihrem Selbstverständnis generell stark nach Politiklogiken operieren. Sie wählen Themen anhand des Relevanzkriteriums der Tragweite der politischen Geschäfte aus, und sie erachten die Kontroll-, Einordnungs- und Aufklärungsfunktion als ihr Ziel. Gleichwohl handeln sie auch nach Präferenzen wie Konfliktivität und Kontroverse, Neuigkeitswert und Exklusivität, sowie auch der Rezeptivität des Publikums für Themen. Diese Befunde stützen die Hypothese H9.

\subsubsection{Zusammenführung der Ergebnisse}

In Unterunterabschnitt 5.1.3 habe ich die qualitativen und quantitativen Ergebnisse derart zusammengefasst, dass ich jeweils angegeben habe, für wie wahrscheinlich ich die Gültigkeit der jeweiligen Hypothese gegeben der verfügbaren Evidenz halte. In Tabelle 28 ergänze ich diese Einschätzung nun noch um die Hypothesen H7, H8 und H9.

In Hypothese $\mathrm{H} 7$ postuliere ich, dass Parlamentarierinnen und Parlamentarier medialisiert nur als Mittel zum Zweck der Politiklogiken handeln. Das Gewicht der Evidenz aus den qualitativen Interviews erachte ich als derart gross, dass ich fast sicher bin, dass diese Hypothese nicht zutrifft. Die Heftigkeit dieser Einschätzung zeugt vielleicht von einer verzerrten Erwartungshaltung, welche zu dieser Hypothese geführt hat. Es ist, im Nachhinein betrachtet, nämlich vielleicht etwas unrealistisch, die Hypothese überhaupt so aufzustellen. Bei der Beurteilung der Ergebnisse zu Hypothese H8 schwingen die unterschiedlichen Teilergebnisse mit. Hypothese H8 postuliert, dass Medien am stärksten über die Geschäfte des National- und des Ständerates berichten, gefolgt von Vorstössen, und am wenigsten über die Fragestunden. Die Ergebnisse legen einerseits sehr deutlich nahe, dass die Medien am stärksten und am konsistentesten über 
Tabelle 28: Zusammenfassung der Hypothesen H1 bis H9.

\begin{tabular}{llll}
\hline \multirow{2}{*}{ Hypothese } & \multicolumn{3}{c}{ Ergebnis } \\
\cline { 2 - 4 } & quantitativ & qualitativ & total \\
\hline H1 & 0.3 & 0.2 & 0.25 \\
H2 & 0.3 & 0.2 & 0.25 \\
H3 & 0.7 & 0.9 & 0.8 \\
H4 & 0.8 & 0.9 & 0.85 \\
H5 & 0.7 & 0.6 & 0.65 \\
H6 & - & - & 0.225 \\
H7 & - & 0.1 & 0.1 \\
H8 & 0.3 & - & 0.3 \\
H9 & 0.3 & 0.8 & 0.55 \\
\hline
\end{tabular}

Die Werte geben an, für wie wahrscheinlich ich die Gültigkeit der Hypothesen gegeben der Evidenz halte. 0 bedeutet, dass ich die jeweilige Hypothese mit der jeweiligen Evidenz als sicher ungültig erachte; 1 bedeutet, dass ich die jeweilige Hypothese mit der jeweiligen Evidenz als sicher gültig erachte.

die Geschäfte des National- und Ständerates berichten. Andererseits deuten die Ergebnisse auch darauf hin, dass Medien, anders als in der Hypothese H8 postuliert, stärker über Fragestunden als über eingereichte Vorstösse berichten. Die Wahrscheinlichkeit, dass Hypothese H8 gültig ist, setze ich aber dennoch nicht maximal tief, weil das Teilergebnis, welches die Widerlegung der Hypothese H8 nahelegt, möglicherweise eine Folge der unterschiedlichen quantitativen Modellierungen ist. Um nämlich zu prüfen, ob Fragestunden die Medienagenda des nächsten Tages beeinflussen, vergleiche ich lediglich die Agenda der Fragestunde mit der Agenda der Medien vom Folgetag. Den Einfluss der Geschäfte des National- und des Ständerates sowie der eingereichten Vorstösse modelliere ich hingegen alle gemeinsam in Zeitreihe-Modellen. Es ist nicht auszuschliessen, dass die Effekte der Fragestunden geringer ausgefallen wären, hätte ich auch in den Modellen zu den Fragestunden die Agenden des National- und des Ständerates sowie der eingereichten Vorstösse eingebaut.

Die Differenz zwischen meiner Einschätzung der qualitativen und der quantitativen Evidenz zu Hypothese H9 ist gross. Hypothese H9 postuliert, dass Journalistinnen und Journalisten in gleichem Masse exklusiv nach Medienlogiken und zusätzlich auch nach Politiklogiken handeln. Die quantitativen Zeitreihe-Modelle haben allerdings den Schluss nahegelegt, dass die Parlamentsberichterstattung in erster Linie durch das Geschehen im National- und im Ständerat bedingt ist. In den qualitativen Interviews hingegen hat sich recht deutlich gezeigt, dass Journalistinnen und Journalisten zumindest in ihrer Selbstwahrnehmung eine Balance halten zwischen Präferenzen, welche sich mit den Präferenzen der Bürgerinnen und Bürger, 
also den Politiklogiken, überschneiden und Präferenzen, welche nur die Präferenzen von Journalistinnen und Journalisten selber umfassen, also Medienlogiken ohne Schnittmenge mit den Politiklogiken. Die Wahrscheinlichkeit, dass Hypothese H9 gegeben der qualitativen Evidenz gültig ist, setze ich trotzdem nicht maximal hoch an, weil in den Interviews ein leichter Überhang zugunsten der Politiklogiken vorhanden ist.

Die in Tabelle 28 festgehaltenen Wahrscheinlichkeiten, oder, anders formuliert, die Grade der subjektiven Gewissheiten ob der Gültigkeit der Hypothesen gegeben der Evidenz, umfassen ein breites Spektrum, von 0.1 bis 0.85 . In einer simplen, binären Unterscheidung der Ergebnisse nach «widerlegt» und «bestätigt» wären vier Hypothesen bestätigt, fünf widerlegt. Eine solche Dichotomisierung macht nach meinem Dafürhalten aber keinen Sinn. Hypothese H9 beispielsweise würde als bestätigt gelten, obwohl ihre explizit eingeschätzte Wahrscheinlichkeit mit 0.55 näher an der Wahrscheinlichkeit der widerlegten Hypothese H8 denn an der bestätigten Hypothese H4 liegt.

\subsection{Diskussion}

\subsubsection{Forschungsfrage 1: Präferenzen im Parlament}

Die erste Forschungsfrage fragt nach dem Verhältnis von Politiklogiken zu Medienlogiken in dem Handeln von Parlamentarierinnen und Parlamentariern:

- FF1: In welchem Masse handeln Parlamentarierinnen und Parlamentarier nach Politiklogiken und in welchem Masse nach Medienlogiken?

Um diese Forschungsfrage zu beantworten, habe ich sieben Hypothesen geprüft. In der ersten Hypothese $\mathrm{H} 1$ habe ich postuliert, dass das Abstimmungsverhalten im Nationalrat nicht durch die mediale Berichterstattung zu den Geschäften, über welche abgestimmt wird, beeinflusst wird. Sowohl die qualitativen wie auch die quantitativen Ergebnisse zu Hypothese H1 sprechen gegen diese. Darum erachte ich die Wahrscheinlichkeit, dass Hypothese H1 gültig ist, mit einem Wert von 0.25 als tief - und damit die Wahrscheinlichkeit, dass die Hypothese ungültig ist, mit 0.75 als hoch. In der zweiten Hypothese H2 postuliere ich dasselbe wie in Hypothese H1, nur auf den Ständerat bezogen. Aus der verfügbaren Evidenz zu Hypothese H2 schliesse komme ich zu demselben Schluss wie für Hypothese H1: Die Wahrscheinlichkeit, dass Hypothese H2 zutrifft, erachte ich gegeben der Evidenz als sehr unwahrscheinlich, nämlich 0.25; die Wahrscheinlichkeit, dass Hypothese H2 ungültig ist, sehe ich mit 0.75 als eher hoch. In Hypothese H3 postuliere ich, dass die während einer Session eingereichten Vorstösse durch Berichterstattung 
beeinflusst sind. Für Hypothese H3 lese ich die Evidenz deutlich zugunsten der Hypothese und gehe davon aus, dass die Wahrscheinlichkeit, dass Hypothese H3 zutrifft, bei einem hohen Wert von 0.8 liegt. Als noch ein Stück deutlicher erachte ich die Evidenz zu Hypothese H4, welche postuliert, dass die Fragen in den Fragestunden durch Berichterstattung beeinflusst sind: Die Wahrscheinlichkeit, dass Hypothese H4 gültig ist, setze ich mit 0.85 recht hoch an. Mit der Hypothese $\mathrm{H} 5$ prüfe ich, ob die Fragestunden wie vermutet stärker durch mediale Berichterstattung beeinflusst sind als die eingereichten Vorstösse. Auch diese Hypothese erachte ich als durch die empirische Evidenz gestützt, aber nur mit einer Wahrscheinlichkeit von 0.65, da die Ergebnisse zu dieser Hypothese mit einem nicht unbedeutenden Mass an Ungewissheit behaftet ist.

Zur Beantwortung der ersten Forschungsfrage sind vor allem die Hypothesen H6 und H7 von Bedeutung. Mit der Hypothese H6 postuliere ich, dass Parlamentarierinnen und Parlamentarier stärker nach den reinen Politiklogiken denn nach den reinen Medienlogiken sowie der Schnittmenge der Politik- mit den Medienlogiken handeln. Das Prüfen der Hypothese H6 besteht lediglich in einem einfachen Aggregieren der Wahrscheinlichkeiten der Hypothesen H1 bis H4. Das Ergebnis dieser zusammenfassenden Aggregation ist eine Wahrscheinlichkeit von lediglich 0.225 für die Hypothese H6. Somit erachte ich die Wahrscheinlichkeit, dass Parlamentarierinnen und Parlamentarier stärker nach den reinen Politiklogiken handeln, als tief. Mit der Hypothese H7 prüfe ich in Ergänzung zu Hypothese H6, ob jener Teil des Handelns der Parlamentarierinnen und Parlamentarier, welcher nicht aus den reinen Politiklogiken besteht, ausschliesslich aus der Schnittmenge der Politik- und der Medienlogiken besteht. Die Wahrscheinlichkeit, dass Hypothese H7 zutrifft, erachte ich gegeben der starken Evidenz mit einem Wert von 0.1 als sehr tief.

Auf die erste Forschungsfrage liefern die empirischen Analysen zwei konkrete Antworten:

- Die Wahrscheinlichkeit, dass Parlamentarierinnen und Parlamentarier ausschliesslich nach reinen Politiklogiken handeln, beträgt gegeben der Evidenz dieser Arbeit 0.225.

- Die Wahrscheinlichkeit, dass Parlamentarierinnen und Parlamentarier medialisiert ausschliesslich als Mittel zum Zweck der Politiklogiken handeln, beträgt gegeben der Evidenz dieser Arbeit 0.1 .

\subsubsection{Forschungsfrage 2: Präferenzen in den Medien}

Die zweite Forschungsfrage fragt nach dem Verhältnis von Politiklogiken zu Medienlogiken in dem Handeln von Journalistinnen und Journalisten: 
- FF2: In welchem Masse handeln fournalistinnen und fournalisten in der Berichterstattung über das Parlament nach Medienlogiken und in welchem Masse nach Politiklogiken?

Um diese Forschungsfrage zu beantworten, habe ich zwei Hypothesen geprüft. Mit Hypothese H8 habe ich postuliert, dass Medien am intensivsten über die Geschäfte des National- und des Ständerates berichten, gefolgt von den eingereichten Vorstössen und am wenigsten über Fragestunden. Die Wahrscheinlichkeit, dass diese Hypothese zutrifft, schätze ich mit 0.3 tief ein. Dies, weil zwar die Bericherstattung zu den Geschäften des National- und des Ständerates am intensivsten ist, die Berichterstattung über die Fragestunden aber stärker als die Berichterstattung über eingereichte Vorstösse ist. Mit der Hypothese H9 habe ich postuliert, dass sich Journalistinnen und Journalisten in ihrem Handeln in gleichem Umfang an Politiklogiken wie an Medienlogiken orientieren. Die Wahrscheinlichkeit, dass diese Hypothese zutrifft, schätze ich mit einer sehr unsicheren Wahrscheinlichkeit von 0.55 ein, und zwar, weil die qualitative und die quantitative Evidenz sehr unterschiedliche Schlüsse nahe legen, was aggregiert in einer Unsicherheit mündet.

Die empirischen Analysen liefern eine konkrete Antwort auf die zweite Forschungsfrage.

- Die Wahrscheinlichkeit, dass Journalistinnen und Journalisten im Kontext der Parlamentsberichterstattung stärker nach reinen Medienlogiken handeln als nach der Schnittmenge von Medienlogiken und Politiklogiken, beträgt gegeben der Evidenz dieser Arbeit:

$$
\frac{(1-0.3)+(1-0.55)}{2}=0.575
$$

\subsubsection{Forschungsfrage 3: Wandel}

Die dritte Forschungsfrage fragt nach dem Wandel der Befunde zu den ersten zwei Forschungsfragen im Zuge der Medienkrise:

- FF3: Wandeln sich die Präferenzen von Parlamentarierinnen und Parlamentariern sowie von fournalistinnen und fournalisten im Zuge der Medienkrise?

Für diese Forschungsfrage habe ich keine Hypothesen formuliert und geprüft, sondern gehe lediglich an dieser Stelle in explorativer Manier auf sie ein. Eine Annäherung - aber keine zuverlässige bzw. plausible Beantwortung - an die dritte Forschungsfrage ist anhand zweier Datengrundlagen möglich. Erstens können die quantitativen Auswertungen, welche ich zum Zwecke der Beantwortung der Hypothesen durchgeführt habe, insofern verglichen werden, als jede Auswertung in dreifacher Ausführung erfolgt, nämlich für 1999, für 2005 und für 
2011. Zweitens können die qualitativen Interviews Anhaltspunkte darüber liefern, was für Veränderungen die Parlamentarierinnen und Parlamentarier sowie die Journalistinnen und Journalisten für den Zeitraum der Medienkrise wahrnehmen.

Bei den ersten beiden Hypothesen hat sich gezeigt, dass der potenzielle Einfluss der Berichterstattung auf das Abstimmungsverhalten für die Jahre 1999 und 2005 wahrscheinlich ist, für 2011 hingegen weniger. Dies kann ein blosses Artefakt der Fallzahlen sein; 2011 fanden in der Herbstsession lediglich 36 Gesamt- und Schlussabstimmungen im National- und Ständerat statt. Mit einer Fallzahl von 40 fanden in der Herbstsession 2005 aber nicht bedeutend mehr Gesamtund Schlussabstimmungen statt, die Effekte sind aber klarer. Bei der Hypothese H3 fällt auf, dass ein wahrscheinlicher Einfluss der Medien auf die eingereichten Vorstösse sowohl 1999 als auch 2005 vorhanden ist, nicht aber 2011. Dieses Muster bereits als Wandel zu interpretieren, dürfte wohl nicht korrekt sein, nicht zuletzt, weil der Effekt für 1999 schwächer als der Effekt für 2005 ist. Somit handelt es sich nicht um eine lineare Abnahme des Effektes über die Zeit. Für die Hypothese H4 ist zwar zu beobachten, dass die Effekte nicht für alle Fragestunden und für alle Jahre gleich ausfallen, aber die Effekte bleiben gleich umfangreich und gleich stark. Die Ergebnisse der Analysen für die Hypothese H8 deuten ebenfalls nicht auf Veränderungen im Zuge der Medienkrise: Für alle drei Jahre bleiben die Effekte des National- und des Ständerates in etwa gleich gross und haben in etwa gleich tiefe p-Werte. Auch die Effekte der Fragestunden auf die Berichterstattung im Kontext der Hypothese H8 können kaum als Wandel interpretiert werden, da ein wahrscheinlicher Effekt für 1999 und 2011 zu beobachten ist. Die Ergebnisse der Modelle im Kontext der Hypothese H9 könnten auf den ersten Blick Anlass zur Vermutung geben, dass ein Wandel stattfindet: Die Medienagenda ohne Parlamentsberichterstattung übt in einer Woche im Jahr 2011 einen wahrscheinlichen und grossen Effekt auf die Medienagenda mit Parlamentsberichterstattung aus. Der Eindruck eines möglichen Wandels wird durch den Umstand verstärkt, dass im Kontext der Hypothese H9 auch in einer Woche für 2005 ein potenzieller Effekt vorhanden ist, und zwar ein schwächerer, aber auch ein unwahrscheinlicherer. Dies regt zu der ad hoc-Hypothese an, dass 1999 noch keine Effekte vorhanden sind, 2005 schwache und 2011 starke. Diese ad hoc-Hypothese wäre allerdings erst dann plausibel, wenn ein solches Muster für alle drei Wochen pro Jahr beobachtbar wäre und nicht bloss für eine Woche pro Jahr.

Aus den quantitativen Analysen lassen sich also keine plausiblen Veränderungen im Zuge der Medienkrise feststellen. Im Rahmen der qualitativen Interviews haben allerdings sowohl Parlamentarierinnen und Parlamentarier wie auch Journalistinnen und Journalisten einige Entwicklungen in den letzten knapp 15 Jahren beschrieben. Die mit Abstand am häufigsten 
geschilderte Veränderung ist die Wahrnehmung, dass Medien viel Qualität eingebüsst haben. Als Ursachen für die Qualitätseinbussen der Parlamentsberichterstattung werden unterschiedliche Gründe genannt. Einer davon ist die Zunahme des Zeitdrucks bzw. die Verschlechterung der Arbeitsbedingungen für Journalistinnen und Journalisten. Eine Journalistin erklärt diesen gestiegenen Zeitdruck mit dem hohen Publikationsrhythmus der Online-Nachrichtenseiten:

Das hat sicher mit der Schnelligkeit etwas zu tun, oder? Früher hatte man einfach mehr Zeit. Mit diesem ganzen Online-Journalismus, das geht sofort aufs Netz. Der Druck auch von intern, also, dass man schnell arbeiten muss.

Die gestiegene Arbeitsbelastung hängt aber auch mit der personellen Situation zusammen. So beschreibt ein Journalist, dass noch vor weniger Jahren der gleiche Umfang an Arbeit durch mehr Personen erledigt wurde:

Ja, also ich möchte jetzt nicht sagen, dass vor 3 Jahren einfach alles viel, viel besser war. Aber ja, es ist klar, wenn wir etwas mehr Leute wären, hätten wir auch mehr Zeit, uns mit der Materie zu beschäftigen. Und das wäre für die Qualität sicher förderlich. Es gibt halt einfach wirtschaftliche Realitäten, die halt so sind, wie sie sind.

Die personelle Situation beschreibt auch eine weitere Journalistin als ein Problem, dass sich vor allem bei privaten Medien bemerkbar macht:

Es hängt natürlich damit zusammen, dass die Privaten sehr schlecht zahlen. Also nehmen sie, müssen sie relativ junge, nicht gut ausgebildete Leute nehmen, die dann logischerweise auch nicht ewig bleiben. Und im Arbeitsalltag müssen die noch viel viel viel schneller - noch viel schneller produzieren. Also das ist eine ziemlich komplexe Angelegenheit.

Ein Mitglied des Nationalrates erklärt, dass diese oftmals jungen Journalistinnen und Journalisten zwar prinzipiell kompetent sind, aber nicht die Funktion haben, sich vertiefte DossierKenntnisse anzueignen, sondern eher, rasch Output zu generieren:

Die können einem nicht erklären was zwischen dem ersten und zweiten Weltkrieg passiert ist. Die können einem nicht genau erklären DDR/BRD und sofort. Das sind zwar gescheite Leute, die ein Gymnasium abgeschlossen haben, haben irgendwie Medien-Kommunikation studiert drei Jahre, haben den Bachelor und jetzt los. 
Ein Journalist bestätigt diese Sichtweise und argumentiert, dass junge Journalistinnen und Journalisten heute grundsätzlich andere Biographien aufweisen:

Die haben meistens irgendeinen Bachelor gemacht, oder irgendwas, äh, Master in weiss nicht was gemacht und - was es früher, glaub ich, in dieser Form nicht gegeben hat - arbeiten dann schon bei der SonntagsZeitung, beim Blick, beim wo auch immer, Tages-Anzeiger, NZZ, auch die machen das im Übrigen; alle Verlage, aus Kosten-, Spardruck. Und es war früher wirklich nicht so, da hat man eben noch den klassischen Weg über die Lokalzeitung vielleicht gemacht. Oder in der Regionalzeitung eben war man lange Volontär und musste lernen und vielleicht nebenan noch eine Journalistenschule besuchen.

Die Kombination der gestiegenen Arbeitslast mit dem demografischen Wandel der Redaktionen hat zur Folge, so das Argument einer Reihe von Parlamentarierinnen und Parlamentariern wie auch von Journalistinnen und Journalisten, dass es zu einem Kompetenzschwund kommt. Eine Journalistin erklärt beispielsweise, dass diese Jungjournalistinnen und -journalisten rein fachlich mit dem Parlamentsgeschehen bisweilen überfordert sind:

Und was ich vorhin gesagt habe mit der Fachkompetenz. Also ich höre wirklich manchmal Interviews von jungen KollegInnen unter extremem Zeitdruck, die wirklich die Botschaft zum Thema nicht gelesen haben, auch nicht gelesen haben können. Ich werfe das ihnen überhaupt nicht vor, sondern sie tun mir dann auch Leid. Und die müssen sich dann wirklich sehr viel erklären lassen. Und so diese stressige Session, auch für die Parlamentarier, und dann hineinrennen, und dann die Verpflichtung, die sie sonst haben, und dann jemandem die ganze Gesundheitsökonomie erklären. Ich glaube, das gibt dann vielleicht so ein bisschen eine Unruhe. So würde ich das beschreiben.

Eine Parlamentarierin beschreibt mit sehr deutlichen Worten ihre Erfahrungen mit solch jungen, fachlich nicht sehr fundierten Journalistinnen und Journalisten:

Dann hat die Qualität der Journalisten mit diesen Gratiszeitungen massiv abgenommen. Wirklich massiv. Ich erlebe es regelmässig, dass mich Journalisten, auch aus ganz wichtigen Zeitungen, anrufen und fragen, um was es da genau ginge und anschliessend einen kleinen Artikel darüber schreiben, aufgrund dessen, was ich ihnen erklärt habe. Denn sie haben keine Ahnung. Sie haben wirklich keine Ahnung von der Materie. 
Ein Mitglied des Ständerates betont, dass Journalistinnen und Journalisten mit langjähriger Erfahrung wichtig sind, weil mit der Erfahrung auch das Wissen, also die Kompetenz zunimmt:

Und man stellt das immer wieder fest, dass ja bei einzelnen Medien es langjährige Mitarbeitende gibt und bei anderen Medien, jedes Mal eine andere Person erscheint. Und das ist auch ein Zeichen der Kompetenz und der Kontinuität. Und diese [Journalisten] sind wirklich; denen ist ein Kranz zu binden, die wissen dann auch wie sie die Ergebnisse zu kommunizieren haben.

Eben diese Journalistinnen und Journalisten verlassen aber zunehmend Medien und wenden sich der PR-Branche zu. So bemerkt ein Mitglied des Nationalrates etwa, dass die Bundesverwaltung ein beliebter Arbeitgeber sei:

Und Qualitätsjournalisten verlassen auch die Medienhäuser. Also die gehen. Es sind zahlreiche Abgänge zu irgendwelchen, meistens sind es Bundesämter, die dann einfach, ja, die sind dann einfach verloren für den Journalismus, auf immer, die kommen nicht mehr zurück, ja. Es ist sehr schade.

Eine sehr ähnliche Beobachtung macht auch ein Journalist:

Ja genau, [PR-Branche] so, oder Sprecher eines - also das ist ja das, was viele jetzt bei uns machen, in meinem Alter. Sie gehen als Sprecher zu einem Bundesamt, oder bei einem Verband machen sie eben PR-Arbeit, Kommunikation.

Praktisch alle Parlamentarierinnen und Parlamentarier sowie Journalistinnen und Journalisten nehmen Qualitätseinbussen in der Parlamentsberichterstattung infolge grosser struktureller und personeller Umbrüche wahr - sie beschreiben also die Medienkrise und die Folgen der Medienkrise auf der inhaltlichen Ebene des journalistischen Outputs. Das drängt eine offensichtliche Frage auf: Warum äussert sich die Medienkrise, so, wie die betroffenen Akteure sie wahrnehmen, in den inhaltsanalytischen Daten überhaupt nicht? Hierfür gibt es, so meine ich, mehrere Gründe. Erstens sind die Stichprobenjahre, welche ich verwende, nicht gut geeignet, um die Medienkrise abzugreifen. Es handelt sich um nur drei zeitliche Datenpunkte, also 1999, 2005 und 2011, was schlicht zu wenig ist, um plausible Aussagen über die zeitliche Entwicklung machen zu können. Die Datenpunkte liegen zudem auch relativ weit auseinander, wobei der jüngste Datenpunkt das Jahr 2011 ist - bereits zum Zeitpunkt des Verfassens der vorliegenden Arbeit im Jahr 2015 ist die Stichprobe veraltet, wenn es darum geht, den rasanten Verlauf der Medienkrise zu messen. 
Zweitens geht aus den Einschätzungen der Parlamentarierinnen und Parlamentarier sowie der Journalistinnen und Journalisten hervor, dass die inhaltlichen Folgen der Medienkrise in der Qualität der Berichterstattung zu verfolgen sind, nicht zwingend in der blossen Quantität. Die Inhaltsanalyse der vorliegenden Arbeit wurde zwar auch qualitativ durchgeführt, aber nur mit dem Ziel, die Politikfelder und die konkreten Themen zu identifizieren. Die Art und Weise, wie diese Politikfelder und Themen in den Medien präsentiert werden, erschliesst sich aus der durchgeführten Inhaltsanalyse nicht.

Drittens, und vielleicht am wichtigsten: Der Umstand, dass die in dieser Arbeit umgesetzte Inhaltsanalyse keine Effekte der Medienkrise zeigt, könnte schlicht auch bedeuten, dass die Medienkrise die generellen Agenda Setting-Dynamiken zwischen Parlament und Medien, wie ich sie untersuche, nicht tangiert - Medienkrise schlägt sich also vielleicht schlicht nicht in den Agenda Setting-Dynamiken, wie sie in der vorliegenden Arbeit untersucht sind, nieder.

Was also ist, zusammengefasst, das Fazit der knappen Exploration zur dritten Forschungsfrage? Es zeigt sich, dass sowohl Parlamentarierinnen und Parlamentarier wie auch Journalistinnen und Journalisten eine Abnahme der Berichterstattungsqualität infolge erschwerter journalistischer Arbeitsbedingungen und des demografischen Wandels der Redaktionen wahrnehmen. Diese Formen der Medienkrise sind potenziell für die Präferenzen der Journalistinnen und Journalisten relevant: Wenn mit weniger Ressourcen mehr Arbeit durch weniger erfahrene und fachkompetente Journalistinnen und Journalisten erbracht werden muss, dann stellt sich die Frage, nach welchen Präferenzen diese neueren Journalistinnen und Journalisten handeln. Die kurze Exploration zur Forschungsfrage 3 gibt Anlass zu der Annahme, dass das journalistische Handeln im Zuge der Medienkrise stärker ausschliesslich nach Medienlogiken denn nach der Schnittmenge von Medienlogiken und Politiklogiken stattfindet. Diese Hypothese muss aber, selbstredend, zunächst in weiterführender Forschung geprüft werden; der Exkurs zu der dritten Forschungsfrage in der vorliegenden Arbeit plausibilisiert höchstens den Entdeckungszusammenhang einer solchen weiterführenden Forschungsarbeit. Für eine solche weiterführende Forschung dürfte die Arbeit mit qualitativen Methoden zielführend sein. Nebst qualitativen Interviews können auch ethnographisch inspirierte Methoden wie etwa die Methode der Beobachtung (Spradley 1980) Prozesse und Dynamiken festhalten, wie sie sich inhaltsanalytisch nur schwer rekonstruieren lassen.

Open Access Dieses Buch wird unter der Creative Commons Namensnennung - Nicht kommerziell 4.0 International Lizenz (http://creativecommons.org/licenses/by-nc/4.0/deed. de) veröffentlicht, welche für nicht kommerzielle Zwecke die Nutzung, Vervielfältigung, Bearbeitung, Verbreitung und Wiedergabe in jeglichem Medium und Format erlaubt, sofern Sie den/die ursprünglichen Autor(en) und die Quelle ordnungsgemäß nennen, einen Link zur Creative Commons Lizenz beifügen und angeben, ob Änderungen vorgenommen wurden.

Etwaige Abbildungen oder sonstiges Drittmaterial unterliegen ebenfalls der genannten Creative Commons Lizenz, sofern sich aus der Abbildungslegende oder der Quellreferenz nichts anderes ergibt. Sofern solches Drittmaterial nicht unter der genannten Creative Commons Lizenz steht, ist eine Vervielfältigung, Bearbeitung oder öffentliche Wiedergabe nur mit vorheriger Zustimmung des betreffenden Rechteinhabers oder auf der Grundlage einschlägiger gesetzlicher Erlaubnisvorschriften zulässig. 


\section{Schlussbetrachtungen}

\subsection{Zusammenfassung der Ergebnisse}

Die Ausganslage für die vorliegende Arbeit ist die normative Überlegung, dass Bürgerinnen und Bürger in einem besonderen Verhältnis zu Parlamentarierinnen und Parlamentariern stehen. Bürgerinnen und Bürger als Prinzipal beauftragen Parlamentarierinnen und Parlamentarier als Agenten, im Sinne ihrer Präferenzen zu legiferieren. Dieses Prinzipal-Agent-Verhältnis kann aber nur funktionieren, wenn der Prinzipal, also die Bürgerinnen und Bürger, die Möglichkeit haben, die Arbeit des Agenten, also der Parlamentarierinnen und Parlamentarier, öffentlich zu beobachten. Der Agent hat nämlich nicht komplett deckungslgeiche Präferenzen wie der Prinzipal, und wenn der Prinzipal die Arbeit des Agenten nicht kontrollieren, also nicht beobachten kann, dann kann der Prinzipal auch nicht sinnvollerweise entscheiden, ob er die Arbeit des Agenten billigt oder nicht. Öffentliche Beobachtbarkeit des Parlamentes als notwendige Bedingung für das Funktionieren der Prinzipal-Agent-Beziehung nenne ich die kommunikative Legitimität des Parlamentes.

Die öffentliche Sichtbarkeit des Parlamentes und damit dessen kommunikative Legitimität wird in erster Linie durch Massenmedien, also durch Journalistinnen und Journalisten, hergestellt. Damit spielen Medien eine besondere Rolle in dem Prinzipal-Agent-Verhältnis zwischen Bürgerinnen und Bürgern als Wählerschaft und Parlamentarierinnen und Parlamentariern. Die Relevanz der Medien erschöpft sich allerdings nicht in der blossen Funktion des Sichtbarmachens des Parlamentes. Journalistinnen und Journalisten sind selber nämlich ebenfalls handlungsfähige Akteure mit eigenständigen Präferenzen. Somit existiert im Prinzipal-AgentVerhältnis zwischen Bürgerinnen und Bürgern und Parlamentarierinnen und Parlamentariern eine dritte Akteursgruppe, Journalistinnen und Journalisten. Aus diesen drei Akteursgruppen ergeben sich zwei Präferenzfamilien, welche zueinander teils in einem ausschliessenden Verhältnis stehen. Die Präferenzfamilie der Politiklogiken umfasst all jene Präferenzen, welche Bürgerinnen und Bürger und Parlamentarierinnen und Parlamentarier teilen sowie zusätzlich die Schnittmenge der Präferenzen, welche alle drei Akteursgruppen teilen. Die Präferenzfamilie der Medienlogiken umfasst all jene Präferenzen, welche Journalistinnen und Journalisten und Parlamentarierinnen und Parlamentarier, nicht aber Bürgerinnen und Bürger teilen. Gegeben der theoretischen Logiken dieser Schnittmengen in der Prinzipal-Agent-Beziehung sowie der empirischen Beobachtung, dass sich das Mediensystem der Schweiz seit ca. Beginn der 2000er Jahre in einer strukturellen Medienkrise befindet, ergeben sich folgende drei Forschungsfragen:

- FF1: In welchem Masse handeln Parlamentarierinnen und Parlamentarier nach Politiklogi- 
ken und in welchem Masse nach Medienlogiken?

- FF2: In welchem Masse handeln fournalistinnen und fournalisten in der Berichterstattung über das Parlament nach Medienlogiken und in welchem Masse nach Politiklogiken?

- FF3: Wandeln sich die Präferenzen von Parlamentarierinnen und Parlamentariern sowie von fournalistinnen und fournalisten im Zuge der Medienkrise?

Gestützt auf die theoretischen Ansätze von Agenda Setting, Medialisierung und Rational ChoiceInstitutionalismus habe zur Beantwortung der ersten Forschungsfrage folgende sieben Hypothesen aufgestellt:

- Hypothese H1: Abstimmungen im Nationalrat sind nicht durch die vorangehende Berichterstattung zu den betroffenen Geschäften beeinflusst.

- Hypothese H2: Abstimmungen im Ständerat sind nicht durch die vorangehende Berichterstattung zu den betroffenen Geschäften beeinflusst.

- Hypothese H3: Die eingereichten Vorstösse sind durch die vorangehende Berichterstattung beeinflusst.

- Hypothese H4: Die Fragen in den Fragestunden des Nationalrates sind durch die vorangehende Berichterstattung beeinflusst.

- Hypothese H5: Fragen in den Fragestunden sind stärker durch Berichterstattung beeinflusst als die eingereichten Vorstösse.

- Hypothese H6: Parlamentarierinnen und Parlamentarier handeln stärker nach Politiklogiken als nach Medienlogiken.

- Hypothese H7: Parlamentarierinnen und Parlamentarier handeln medialisiert als Mittel zum Zweck zugunsten der Politiklogiken.

Zur Beantwortung der zweiten Forschungsfrage habe ich folgende weitere zwei Hypothesen aufgestellt:

- Hypothese H8: Medien berichten am meisten über die traktandierten Geschäfte, weniger über die eingereichten Vorstösse und am wenigsten über die Fragestunden im Nationalrat.

- Hypothese H9: Journalistinnen und Journalisten handeln in gleichem Umfang nach Medienlogiken wie nach Politiklogiken.

Diese neun Hypothesen habe ich sowohl quantitativ mit inhaltsanalytischen Daten wie auch qualitativ mit Daten aus teilstrukturierten Leitfadeninterviews geprüft und in Tabelle $28 \mathrm{zu}$ sammengefasst, für wie wahrscheinlich ich die einzelnen Hypothesen gegeben der empirischen 
Evidenz halte. Die Hypothesen H1 und H2 erachte ich mit je einem Wert von 0.25 als sehr wahrscheinlich nicht wahr: Sowohl im National-, wie auch im Ständerat gibt es quantitative und qualitative Anzeichen dafür, dass das Abstimmungsverhalten durch Berichterstattung in den Medien beeinflusst ist. Die Hypothesen H3 und H4 hingegen erachte ich mit Wahrscheinlichkeiten von 0.8 und 0.85 als sehr wahrscheinlich wahr; es gibt deutliche quantitative wie qualitative Anzeichen dafür, dass sowohl Vorstösse wie auch Fragen in den Fragestunden durch vorangehende Berichterstattung beeinflusst sind. Ebenfalls gibt es empirische Anzeichen dafür, dass die Fragen in den Fragestunden stärker durch Berichterstattung beeinflusst sind als Vorstösse (Hypothese H5); allerdings ist hier die Datenlage nicht über alle Zweifel erhoben und darum die Wahrscheinlichkeit, dass diese Hypothese wahr ist, bei 0.65. Die Hypothese H6, welche postuliert, dass Parlamentarierinnen und Parlamentarier stärker nach Politiklogiken als nach Medienlogiken handeln, erachte ich angesichts der Ergebnisse mit einer Wahrscheinlichkeit von 0.225 als sehr wahrscheinlich falsch. Hypothese H7 besagt, dass Parlamentarierinnen und Parlamentarier dann medialisiert handeln, also dann im Sinne der Präferenzfamilie der Medienlogiken handeln, wenn sie damit gleichzeitig zugunsten der Politiklogiken handeln. Mit einem Wert von lediglich 0.1 erachte ich diese Hypothese als fast definitiv widerlegt. Die Einzelergebnisse für die Hypothesen H1 bis und mit H7 liefern aggregiert zwei Antworten auf die erste Forschungsfrage:

- Die Wahrscheinlichkeit, dass Parlamentarierinnen und Parlamentarier ausschliesslich nach reinen Politiklogiken handeln, beträgt gegeben der Evidenz dieser Arbeit 0.225.

- Die Wahrscheinlichkeit, dass Parlamentarierinnen und Parlamentarier medialisiert ausschliesslich als Mittel zum Zweck der Politiklogiken handeln, beträgt gegeben der Evidenz dieser Arbeit 0.1 .

Gemeinsam betrachtet bedeuten diese zwei Antworten, dass sich Parlamentarierinnen und Parlamentarier in ihrem Handeln mit sehr hoher Wahrscheinlichkeit nicht nach reinen Politiklogiken richten und dass sie, weiter, fast sicher zugunsten der Medienlogiken handeln, ohne, dass sie sich dabei immer nach den Politiklogiken, also den Präferenzen der Bürgerinnen und Bürger, richten.

Zur Beantwortung der zweiten Forschungsfrage habe ich zwei Hypothesen geprüft. Hypothese H8 postuliert, dass Journalistinnen und Journalisten in ihrer Berichterstattung eine feste Prioritätenliste haben: Am meisten wird über die regulären traktandierten Geschäfte berichtet, gefolgt von eingereichten Vorstössen, und am wenigsten über Fragestunden. Hypothese H8 erachte ich mit einem Wert von 0.3 als recht wahrscheinlich widerlegt an, da sich diese vermutete Rangordnung in der quantitativen Auswertung der inhaltsanalytischen Daten nicht 
manifestiert. Hypothese H9 schliesslich postuliert, dass Journalistinnen und Journalisten im gleichen Umfang nach Politiklogiken wie nach Medienlogiken handeln, dass sie also sowohl Präferenzen hegen, welche auch zugunsten der Bürgerinnen und Bürger sind, wie auch solche Präferenzen, welche nicht den Präferenzen der Bürgerinnen und Bürger entsprechen. Die quantitativen und qualitativen Ergebnisse zu Hypothese H9 liefern widersprüchliche Ergebnisse; darum erachte ich die Wahrscheinlichkeit, dass Hypothese H9 zutrifft, mit einem Wert von 0.55 als sehr ungewiss. Die Ergebnisse zu den Hypothesen H8 und H9 liefern aggregiert die Antwort auf die zweite Forschungsfrage:

- Die Wahrscheinlichkeit, dass Journalistinnen und Journalisten im Kontext der Parlamentsberichterstattung stärker nach reinen Medienlogiken handeln als nach der Schnittmenge von Medienlogiken und Politiklogiken, beträgt gegeben der Evidenz dieser Arbeit 0.575.

Die zweite Forschungsfrage ist weniger eindeutig beantwortet als die erste. Angesichts der Ergebnisse ist es nämlich nur leicht wahrscheinlicher, dass Journalistinnen und Journalisten sich lediglich nach Medienlogiken richten und nicht nach der Schnittmenge von Medienlogiken und Politiklogiken.

Um die dritte Forschungsfrage zu beantworten, habe ich keine Hypothesen formuliert, sondern die quantitativen und die qualitativen Daten explorativ reflektiert bzw. analysiert. Bei den quantitativen Ergebnissen spricht nichts für einen kriseninduzierten Wandel. Die in den jeweiligen Sessionen beobachteten Dynamiken zwischen den Aktivitäten im Parlament und der Berichterstattung in den Medien sind zwar nie über alle Stichprobenjahre hinweg identisch. Die teilweise vorhandenen Unterschiede bei den Wechselwirkungen deuten allerdings nicht auf einen Wandel über die Zeit, eher auf ein Rauschen als Folge der Kontingenz der untersuchten Wechselwirkungen. Anders sieht es bei Ergebnissen der explorativen Analyse der qualitativen Daten, der teilstrukturierten Leitfadeninterviews, aus. Zwar handelt es sich bei den qualitativen Einschätzungen der Interviewpartnerinnen und -partner um subjektive Einschätzungen, aber als Einschätzungen, welche von beiden Akteursgruppen getroffen werden, also sowohl von Parlamentarierinnen und Parlamentarierin wie auch von Journalistinnen und Journalisten, ist es nicht verkehrt, diese Einschätzungen ernst zu nehmen. Die von den zwei Akteursgruppen wahrgenommenen Veränderungen umfassen vier wesentliche Punkte:

- Praktisch alle Interviewpartnerinnen und -partner bemerken Qualitätseinbussen in der Parlamentsberichterstattung im Besonderen, in der Politikberichterstattung im Allgemeinen. Diese Qualitätseinbussen äussern sich, so die Einschätzungen, in oberflächlicherer Berichterstattung, kürzeren Beiträgen, weniger Investigativjournalismus. 
- Als eine der Ursachen für die Qualitätseinbussen wird die Zunahme der Arbeitslast für Journalistinnen und Journalisten vermutet. Nicht zuletzt aufgrund des hohen Publikationtsrhythmus in den Online-Medien müssen Journalistinnen und Journalisten mehr Output in weniger Zeit liefern.

- Weiter wird auch ein demografischer Wandel der Redaktionen wahrgenommen. Altgediente Journalistinnen und Journalisten, so die Wahrnehmung, verlassen Medien tendenziell und wechseln zu grossen Teilen in die private oder die staatliche PR-Branche. Redaktionen bestehen zunehmend aus jungen Journalistinnen und Journalisten.

- Dieser Wandel der Redaktionsdemografien in Kombination mit der gestiegenen Arbeitslast führt, so eine weitere Wahrnehmung, zu einem Kompetenzschwund. Junge Journalistinnen und Journalisten haben keine Musse, sich vertieft in einzelne Themen oder Dossiers einzuarbeiten, da sie konstant viel Output liefern müssen.

Das, was die Parlamentarierinnen und Parlamentarier sowie die Journalistinnen und Journalisten in diesen vier miteinander verbundenen Kernpunkten beschreiben, ist recht eigentlich die Medienkrise.

\subsection{Normative Einschätzung der Ergebnisse}

Die normative Ausgangslage der vorliegenden Arbeit ist das in Unterunterabschnitt 2.1.4 hergeleitete Verständnis prozeduralisierter Volkssouveränität als Prinzipal-Agent-Beziehung zwischen den Bürgerinnen und Bürgern als Prinzipal und den Parlamentarierinnen und Parlamentarierin als Agent. Das Parlament geniesst in diesem Verständnis dann und nur dann demokratische Legitimität, wenn der Prinzipal die Möglichkeit hat, die Arbeit des Agenten zu beobachten, um auf Grundlage des Beobachteten bei den Entscheidungsmomenten der Wahlen über den Agenten zu urteilen. Das Parlament als Agent steht also in einer Rechenschaftsbeziehung mit den Bürgerinnen und Bürgern als Prinzipal, und diese Beziehung ist auf die Beobachtbarkeit des Agenten angewiesen. Diese Beobachtbarkeit wird nicht komplett, aber zum grössten Teil durch die öffentliche Kommunikation von Massenmedien hergestellt. Dass das Parlament öffentlich sichtbar ist, ist gegeben. Wie aber genau die öffentliche Sichtbarkeit des Parlamentes zustande kommt, ist nicht klar. Dies ein Stück weit zu prüfen, ist das Ziel dieser Arbeit.

Die normative Aufladung der Prinzipal-Agent-Perspektive kommt durch die handlungstheoretische Konzipierung der betroffenen Akteure zustande. Der normative Auftrag an das Parlament ist es, gemäss den Präferenzen der Bürgerinnen und Bürger zu handeln. Das ist, auf 
der Ebene der einzelnen Handlung einer Parlamentarierin oder eines Parlamentariers, natürlich unmöglich umzusetzen, denn es ist unmöglich, zu jedem Zeitpunkt genau zu wissen, wie die aggregierte Meinung der jeweiligen Wählerschaft aussieht. Darum betrifft die normative Dimension parlamentarischen Handelns nicht zwingend die Entscheidung für oder wider eine bestimmte konkrete Handlung, sondern eher, die generellen Motivstrukturen der Akteure: Warum handeln Parlamentarierinnen und Parlamentarier, wie sie handeln? Im Kontext der öffentlichen Kommunikation zum Parlament gehe ich davon aus, wie in Abbildung 4 visualisiert ist, dass Parlamentarierinnen und Parlamentarier nach unterschiedlichen Präferenzen handeln können. Entweder handeln sie komplett nach der Präferenzfamilie der Politiklogiken, oder komplett nach der Präferenzfamilie der Medienlogiken, oder sie handeln nach einer Schnittmenge der Präferenzfamilien der Politik- und der Medienlogiken. Aus der Prinzipal-Agent-Perspektive ist das normative Ziel, dass Parlamentarierinnnen und Parlamentarier entweder komplett im Sinne der Politiklogiken, oder im Sinne der Schnittmenge von Politik- und Medienlogiken handeln - Parlamentarierinnen und Parlamentarier sollen also so handeln, dass die Politiklogiken zur Geltung kommen, bzw., genauer: Parlamentarierinnen und Parlamentarier sollen so handeln, dass sie davon ausgehen, dass ihr Handeln zugunsten ihrer Wählerschaft ist.

Ein solches normatives Optimum ist, so die Ergebnisse der vorliegenden Arbeit, mit sehr hoher Wahrscheinlichkeit nicht gegeben. Die Wahrscheinlichkeit, dass Parlamentarierinnen und Parlamentarier ausschliesslich nach reinen Politiklogiken handeln, beträgt gegeben der durch die empirischen Analysen zutage geförderten Evidenz lediglich 0.225. Weiter beträgt die Wahrscheinlichkeit, dass Parlamentarierinnen und Parlamentarier medialisiert ausschliesslich als Mittel zum Zweck der Politiklogiken handeln, lediglich 0.1. Diese zwei Einschätzungen quantifizieren nicht genau, wie hoch der Anteil des normativ problematischen Handelns der Parlamentarierinnen und Parlamentarier ist, denn sie beschreiben in erster Linie, als wie aussagekräftig und allgemeingültig ich die zutage geförderte Evidenz einschätze. Trotz der mit den Ergebnissen verbundenen Unsicherheiten bleibt aber festzustellen, dass in Anbetracht des empirischen Gesamtbildes ein beträchtlicher Teil des Handelns der Parlamentarierinnen und Parlamentarier zuungunsten der Politiklogiken ausfällt. Und was also bedeutet dieser Befund nun? Er ist nicht unbedeutend, aber er bedeutet, normativerweise, keinen alarmierenden Befund.

Die vorliegende Arbeit gründet in einer konzeptuell-theoretischen Argumentation, welche nach meinem Dafürhalten eine hohe handlungstheoretische und normative Wichtigkeit besitzt - vielleicht ähnele ich in diesem Punkt dem Coiffeur, welcher meint, dass der Kunde unbedingt einen Haarschnitt braucht - , welche so aber bisher in der Literatur nicht existiert. 
Das bedeutet, dass das, was ich untersuche, die Art, wie ich es untersuche und vor allem der Grund, warum ich es untersuche, in einer ähnlichen Form wie der hier präsentierten bisher nicht umgesetzt wurde. Wenn für eine empirische Arbeit mit starkem normativen Impetus aber kein Vergleich mit anderen, ähnlich gearteten Arbeiten gemacht werden kann, dann sind auch die Ergebnisse dieser einen Arbeit aus normativer Sicht ohne weitere empirische Bezugspunkte. Und ohne zusätzliche empirische Bezugspunkte, egal, ob diese die zeitliche oder die vergleichende oder beide Dimensionen betreffen, ist es kaum möglich, sinnvoll einzuschätzen, welche normative Bedeutung der eine vorliegende Datenpunkt hat. Oder, in anderen Worten ausgedrückt: Normative Konzepte arbeiten immer mit Idealpunkten, aber ohne eine bestimmte Menge an empirischen Informationen ist es es kaum möglich, abzuschätzen, wie erreichbar oder unerreichbar die Ideale sind. Um aber zumindest ansatzweise zu ertasten, ob die normative Rahmung dieser Arbeit aus praktischer Sicht eine Rolle spielt und wenn ja, in welchem Masse und in welcher Art und Weise, habe ich die Parlamentarierinnen und Parlamentarier sowie die Journalistinnen und Journalisten nach ihrer eigenen Einschätzung der Situation rund um das Parlament und Medien gefragt.

\subsubsection{Die praktische Relevanz des Normativen}

Um die normative Einschätzung der Parlamentarierinnen und Parlamentarier sowie der Journalistinnen und Journalisten zu der Beziehung zwischen Parlament und Medien in Erfahrung zu bringen, habe ich die Interviewpartner zunächst nach ihrer generellen Einschätzung ob der normativen Lage gefragt. Diese generelle Einschätzung fällt konsensuell aus - die Meinungen zum allgemeinen Verhältnis von Parlament und Medien sind, ohne Ausnahme, dass die Gesamtsituation positiv ist. Ein Mitglied des Nationalrates beschreibt die im Allgemeinen befriedigende normative Situation mit folgenden Worten:

Schauen Sie, grundsätzlich befinden wir uns in der Schweiz natürlich in sehr guten Verhältnissen. Wir haben, glaube ich, ein sehr hohes Niveau an Berichterstattungen. Auch von Gratiszeitungen, Boulevard und so weiter. Wenn Sie Politiker fragen, würden sich diese die Kommunikation immer etwas anders vorstellen, das ist klar. Aber das kann auch nicht der Wertemassstab sein. Medien müssen unabhängig sein. Und Unabhängigkeit bedeutet natürlich, dass man vielleicht mal findet: Das ist jetzt nicht so gut.

In einer solchen Lesart wäre normative Kritik, wie ich sie angesichts der empirischen Ergebnisse äussere, eine Art Jammern auf hohem Niveau - im Sinne, dass die Situation bereits sehr nahe an 
dem empirisch erreichbaren Ideal ist. Ähnlich wie das oben zitierte Mitglied des Nationalrates schätzt ein Journalist die allgemeine Situation ein:

Ich würde sagen, wir sind noch im grünen Bereich. Und ich würde auch sagen, wenn wir das schweizerische Mediensystem - es gibt viel Kritik an den Schweizer Medien, auch von den Politikern und so weiter, die ist zum Teil berechtigt, zum Teil halte ich sie für nicht berechtigt - , aber wenn wir unser System vergleichen mit gewissen ausländischen Mediensystemen, dann muss ich sagen, können sich eigentlich auch die Politiker nicht so stark beklagen. Da trägt natürlich auch die direkte Demokratie bei. Ich glaube, es gibt kaum ein Land, wo die Medien so intensiv über sachpolitische Themen schreiben.

Aus der Einschätzung dieses Journalisten wie auch aus der Einschätzung des oben zitierten Mitgliedes des Nationalrates wird das klare Argument ersichtlich, dass die Situation in der Schweiz im internationalen Vergleich sehr gut ist. Weitere Akteure beschreiben die generelle Situation kurz und knapp als unproblematisch, etwa ein Mitglied des Nationalrates:

Ich habe nicht grosse Probleme.

Obschon die generelle Situation also als positiv beurteilt wird, sehen sowohl Parlamentarierinnen und Parlamentarier als auch Journalistinnen und Journalisten in einzelnen Teilaspekten Probleme. Am meisten wird der in Unterunterabschnitt 5.2.3 beschriebene Qualitätsverlust als normatives Problem gesehen. Ein Mitglied des Nationalrates beschreibt das Problem mit folgender, emotional geladener Beobachtung:

Was heute ein bisschen entsetzlich ist für mich: Es geht fast nie richtig um Inhalte.

Eine gewisse Oberflächlichkeit als Folge des (kriseninduzierten) Wandels von Redaktionen beklagt auch ein weiteres Mitglied des Nationalrates:

Also was ich mir wünschen würde, ist, dass mehr Zeitungen bessere Hintergrundberichte bringen würden. Jetzt habe ich manchmal den Eindruck, viele Zeitungen bilden einfach mal irgendetwas ab. Sie recherchieren zu wenig, was jetzt genau der Hintergrund dazu ist und ob der Hintergrund dann auch tatsächlich stimmt.

Ein anderes Mitglied des Nationalrates sieht im Kontext der Medienkrise nicht zwingend die womöglich sinkende Güte der Berichterstattung als Problem, sondern eher die Medienkonzentration: 


\begin{abstract}
Also wir stellen ganz klar den Trend fest, dass wenn wir mit einem Vorschlag im Blick am Abend oder im 20Minuten sind, kriegen wir ein grösseres Echo, als wenn wir in einer Tageszeitung sind. Also offensichtlich wird das mehr durchgeblättert. Das ist sicherlich auch eine Folge der Konzentration dieser Medienhäuser, dass es möglich ist, dass man diese Gratiszeitschriften weiterhin aufrechterhält und suggeriert, das wären die wirklich wichtigen Themen, mit denen sich das Land und das Parlament befasst. Konzentration der Medienseite finde ich schon problematisch. Führt dann zu einer Qualität an Berichterstattung, die mir persönlich Mühe bereitet.
\end{abstract}

Aus dieser Sicht ist Medienkonzentration nicht die Folge eines funktionierenden Marktes, sondern die Folge eines dysfunktionalen Marktes. Die Medienkonzentration beschreibt auch ein weiteres Mitglied des Nationalrates als Problem, und zwar hinsichtlich der abnehmenden Meinungsvielfalt:

Ja also ich glaube eine massive Medienkonzentration. Ich meine, heute steht im Bund dasselbe wie im Tages-Anzeiger, also nicht auf den Lokalseiten. Allein die Redaktion, die Bundeshausredaktion, Bund, Tagi, man weiss genau,[...] das ist ein bisschen Mischung und alles andere wird weggelassen. Die BaZ gibt es nicht mehr wirklich als unabhängiges Blatt, [...], und dann gibt es, ja, halt die Aargauer Zeitung und die Südostschweiz, das ist auch dieselbe Redaktion auf nationaler Ebene.

Diese Meinungsvielfalt erachtet ein anderes Mitglied des Nationalrates als potenzielle Gefahr der «Berlusconisierung»:

Oder demokratisch problematisch wird es erst, wenn wir eine Berlusconisierung haben von der Schweizer Medienlandschaft. Und das ist im Moment ein bisschen der Fall.

Auch ein Journalist empfindet Medienkonzentration als Problem, und zwar, weil die Vielfalt schwindet, aber auch, weil mit der Vielfalt auch die Arbeitsplätze schwinden:

Und im Endeffekt, da sind immer auch Stellen betroffen und das tut einem jedes Mal weh. Das heisst, es gibt weniger Meinungen aus dem Bundeshaus, und weniger Vielfalt. Und das ist relativ selten, dass es mehr gibt, oder. Die Tendenz geht schon in Richtung immer weniger. 
Einige Akteure sehen die Probleme aber nicht nur bei den privaten Medien, sondern auch bei der als Verein organisierten, öffentlichen $\mathrm{SRG}^{41}$. Ein Nationalratsmitglied beschreibt, dass die SRG zu grosse Macht habe, weil sie über zu umfangreiche finanzielle Mittel verfügt:

Was wirklich vielleicht noch für den Staat ein Problem ist, ist im Fernsehen. Also, ich finde einfach, die SRG hat eine unglaubliche Macht. Das ist, das ist krass. Sie hat extrem viel Mittel, sie hat einen Auftrag, wo ich finde, den muss sie auch haben, bei Zeug, das in diesem Land sonst niemand produzieren würde. [...] Aber was sie in der Tat macht, ist, mit dem gigantischen Dampfwalzen-Budget alles überfahren, sich immer breiter ausdehnen. Und das sind Journalisten, die - natürlich sind das auch unabhängige Journalisten, aber sie sind, schlussendlich, beim Staat.

Ein Journalist erachtet die SRG ebenfalls als teilweise problematisch, weil die Berichterstattung zu staatsnah ausfällt:

Und die [Journalisten bei der SRG] sind auch sehr, sehr auf Linie; die berichten immer sehr staatstragend, und sind lange nicht so kritisch. Das werfen wir ihnen vor. Und sie werfen uns sicher anderes vor, dass wir populistisch sind [...].

Die normative Einschätzung der Parlamentarierinnen und Parlamentarier sowie der Journalistinnen und Journalisten hat also zwei Dimensionen. Die erste Dimension betrifft die Meinung zu der allgemeinen, globalen Situation bezüglich des Verhältnisses von Parlament und Medien. $\mathrm{Zu}$ der ersten Dimension fällt die normative Bewertung eindeutig positiv aus: Die Situation in der Schweiz ist, vor allem im internationalen Vergleich, unproblematisch. Einzelne Aspekte dieser Gesamtsituation werden aber als problematisch erachtet. Allen voran die durch die Medienkrise bedingten Umbrüche, etwa die wahrgnommene Abnahme der Qualität der Berichterstattung, oder auch die zunehmende Medienkonzentration. Was für einen Bezug hat das theoretische normative Modell des Prinzipal-Agent-Verhältnisses zu diesen praktischen normativen Bedenken?

Das Modell einer Prinzipal-Agent-Beziehung fokussiert auf die zwei involvierten Akteure (bzw. Akteursgruppen), den Prinzipal und den Agenten. Das Problem der mangelnden Beobachtbarkeit des Agenten, also der Informationsasymmetrie zwischen Prinzipal und Agent, wird dabei eher abstrakt behandelt. Prinzipal-Agent-Modelle verwenden die Informationsasymmetrie also als eine Prämisse des Modells, aber diese Informationsasymmetrie selber bildet nicht den Fokus der Forschung, sondern eher die Frage nach Anreizen und Sanktionen, mit denen

\footnotetext{
$41 \quad$ Schweizerische Radio- und Fernsehgesellschaft.
} 
der Agent «diszipliniert» werden kann. In der Anwendung des Prinzipal-Agent-Modells als normative Grundlage für das Verhältnis von Bürgerinnen und Bürgern zu Parlamentarierinnen und Parlamentariern ist aber das Problem der Informationsasymmetrie nicht mehr einfach eine als konstant erachtete Prämisse, sondern der normative wie auch der empirische Fokus der Forschungsanstrengung. Wenn aber das Problem der Informationsasymmetrie den Kern der Prinzipal-Agent-Konstellation zwischen Wählerschaft und Parlament bildet, dann ist eine Konzeptualisierung von Information von Nöten, welche über die ad hoc-Konzeptualisierung, wie ich sie in dieser Arbeit verwende, hinausgeht. Die normativen Überlegungen der Parlamentarierinnen und Parlamentarier sowie der Journalistinnen und Journalisten liefern zu diesem Zweck wertvollen Input. Das soll nicht bedeuten, dass das theoretisch hergeleitete PrinzipalAgent-Modell mit kontextbezogenen, nicht-generalisierbaren Komponenten angereichert wird. So wäre es etwa verkehrt, eine solche Variante der Prinzipal-Agent-Theorie zu postulieren, welche die aktuelle Medienkrise bereits in die theoretischen Annahmen integriert. Das wäre darum verkehrt, weil die aktuelle Medienkrise einen Satz an raum-zeitlich abgegrenzten sozialen Ereignissen beschreibt, welche eo ipso nicht generalisierbaren Charakter haben. Was aber generalisierbar ist, sind die normativen Kriterien, anhand derer die Parlamentarierinnen und Parlamentarier sowie die Journalistinnen und Journalisten die Medienkrise beurteilen. Es handelt sich dabei um zwei Kernkriterien:

- Die Qualität der Berichterstattung.

- Die Vielfalt der Berichterstattung.

Die Qualität der Berichterstattung hat, bei näherer Überlegung, einen sehr plausiblen Bezug zu der Problematik der Informationsasymmetrie bei Prinzipal-Agent-Beziehungen. Wenn das normative Ziel nämlich darin besteht, die Informationsasymmetrien zu minimieren, also die Beobachtbarkeit des Agenten zu maximieren, dann spielt fast automatisch auch die Güte der Berichterstattung eine Rolle. Um nur ein banalst mögliches Beispiel als Illustration zu nehmen: Ein Artikel in einer Zeitung kann einen Achtel der Zeitungsseite ausmachen, er kann aber aber auch die ganze Seite abdecken. Angenommen, in beiden Fällen wird derselbe Gegenstand thematisiert, ein bestimmtes Geschäft aus dem Nationalrat. Es ist zu erwarten, dass die Menge an Informationen in dem Artikel, welcher die ganze Seite ausmacht, höher ist als die Menge an Informationen im kleineren Artikel. Das bedeutet, dass die absolute Anzahl der Artikel dieselbe ist, dem Prinzipal aber der eine Artikel stärker zum Nutzen gereicht als der andere.

Die Vielfalt der Berichterstattung kann für die Prinzipal-Agent-Beziehung ebenfalls in einer generalisierten Form von hoher Bedeutung sein. Einerseits bedeutet das Ausleuchten 
eines Gegenstandes aus mehreren Blickwinkeln, dass die Form des Gegenstandes tendenziell besser sichtbar wird - das ist denn auch, in stark verkürzter Form, die erkenntnistheoretische Grundlage und Begründung für ein triangulierendes Forschungsdesign, wie ich es in dieser Arbeit anwende.

Für weiterführende Forschung, welche das mit dem Konzept der prozeduralisierten Volkssouveränität als Prinzipal-Agent-Beziehung operiert, oder breiter, für Forschung, welche das Prinzipal-Agent-Modell aus öffentlichkeitstheoretischer Perspektive anzuwenden gedenkt, ist eine konzeptionelle Weiterentwicklung angebracht, welche das Problem der Informationsasymmetrie theoretisch ausbaut. Der Beitrag der vorliegenden Arbeit für diese konzeptionelle Weiterentwicklung besteht in der Erkenntnis, dass die blossen Volumina der Berichterstattung, mit denen ich in dieser Arbeit empirisch operiere, lediglich eine Dimension im Kontext der Informationsasymmetrie darstellen. Zwei weitere sowohl empirisch wie auch normativ plausible Dimensionen, um welche das Verständnis von Information und Informationsasymmetrie angereichert werden kann, sind die Qualität sowie die Vielfalt der Berichterstattung.

\subsection{Weiterführende Forschung}

Die Bemerkung am Ende von Forschungsarbeiten, dass weitere Forschung nötig sei, ist für sich genommen wenig mehr als eine Floskel - es wäre eher einer Bemerkung wert, wenn zu einem bestimmten Thema oder Sachverhalt keine weitere Forschung nötig wäre. Um an dieser Stelle dennoch einigermassen gerechtfertigt Anregungen für zukünftige Forschung zum Thema der Wechselwirkung von Parlament und Medien machen zu dürfen, sei zunächst erwähnt, woraus ich diese Empfehlungen ableite: Aus den Fehlern und Unzulänglichkeiten der vorliegenden Arbeit. Das, was ich an dieser Stelle also empfehle, sind nicht generelle Ideen, was alles grundsätzlich noch beforscht werden könnte, sondern spezifischer Dinge, welche aus der Forschungsperspektive der vorliegenden Arbeit verbesserungswürdig sind. Anregungen für eine theoretische Weiterentwicklung habe ich bereits in dem vorangehenden Unterunterabschnitt 6.2.1 formuliert; an dieser Stelle sind konkrete forschungsprogrammatische Aspekte von Interesse.

In Betreff des Forschungsdesigns ist das grösste Manko der vorliegenden Arbeit, dass ich für die Medienstichprobe ausschliesschlich deutschsprachige Titel untersucht habe, während das Parlamentsgeschehen eine Totalerhebung darstellt. Dieses asymmetrische Vorgehen ist ein Problem nicht zuletzt, weil sich die parlamentarischen Aktivitäten während der Session nicht sinnvollerweise nach Sprachregionen trennen lassen. Die offensichtliche Lösung ist, nebst Deutschschweizer Medien zusätzlich auch Medien aus der Romandie wie auch aus dem Ticino 
in eine zukünftige Stichprobe miteinzubeziehen. So offensichtlich diese Lösung ist, so heikel ist sie auch. Eine zukünftige Stichprobe auf alle Schweizer Sprachregionen auszuweiten bedeutet nämlich, dass bedeutend mehr Ressourcen für die Datenerhebung und -auswertung eingesetzt werden müssen.

Nebst einer Erweiterung der Medienstichprobe auf alle Sprachregionen ist auch eine Ausweitung der erfassten Medientypen von Nöten. Auch die Online-Berichterstattung der Zeitungsverlage sowie der Fernseh- und Radiosender sollte erfasst werden. Für gewisse Fragestellungen dürften die gedruckten Zeitungsausgaben und ausgestrahlten Sendungen nach wie vor im Sinne der pars pro toto-Logik repräsentieren, welche Themen in den Medien generell wie prominent sind. Das spezifische auf Agenda-Setting bezogene Design in der vorliegenden Arbeit ist aber in zeitlicher Hinsicht sehr sensibel: Ich habe quantitativ Zeitreihen analysiert, bei welchen die gegenseitigen Einflüsse auf Tagesebene gemessen werden. Das macht für klassische Medien Sinn, denn die meisten Zeitungen erscheinen nach wie vor am Morgen nach einem Sessionstag, Fernseh- und Radiosendungen am Abend desselben Tages. Bei Online-Medien ist diese klar strukturierte zeitliche Komponente nicht mehr vorhanden, denn online kann Berichterstattung zum Parlament de facto in Echtzeit veröffentlicht werden, was in Form u.a. von Nachrichtentickern bisweilen auch geschieht. Das Auflösen der zeitlichen Einheit des Tages als Ausgangspunkt für Agenda-Setting-Effekte angesichts der Natur der Online-Medien ist in diesem Sinn sogar eine potenzielle neue Forschungsfrage: Über welche Inhalte des Parlamentes wird online wann berichtet?

Das Design-Paradigma der Triangulation hat sich für die vorliegende Arbeit, so meine ich, vollumfänglich bewährt. Gerade bei jenen Ergebnissen, bei denen sich die inhaltsanalytischen und die interviewbasierten Teilergebnisse widersprechen, wäre die Einschätzung der betroffenen Hypothesen mit nur einer der zwei verwendeten methodischen Herangehensweisen stark verzerrt. Ein Punkt im Zusammenhang mit Triangulation, welchen ich bereits in Unterabschnitt 4.4 erwähne, wird bei einer abschliessenden Betrachtung nach der Besprechung der Ergebnisse nochmals deutlicher. Um die Fragestellungen der vorliegenden Arbeit noch plausibler zu beantworten, wäre ein dritter Triangulierungsschritt notwendig gewesen: Die qualitative Analyse einzelner Themenkarrieren im Laufe der Sessionen. Mit der quantitativ ausgewerteten Inhaltsanalyse in der vorliegenden Arbeit werden zwar globale Effekte über eine grosse Datenmenge hinweg ausfindig gemacht, aber um diese über die jeweiligen Zeitreihen generalisierten Dynamiken handlungstheoretisch unmittelbar zu verstehen, ist es nötig, den Lauf einzelner «Themenkarrieren» qualitativ nachzuzeichnen, also hermeneutisch-kausal rückzuverfolgen, wo einzelne Themen ihren Ursprung haben, und, wie sie sich verbreiten. 
Das Auslassen dieses dritten Triangulationssschrittes rechtfertige ich in Unterabschnitt 4.4 mit dem Umstand, dass die für ein Forschungsprojekt verfügbaren Ressourcen ein Nullsummenspiel darstellen, und das Einbeziehen weiterer Triangulationsschritt im Sinne von mehr Breite der Analyse demgemäss auf Kosten der Tiefe der Analyse stattfindet. An dieser banalen Realität der begrenzten Forschungsressourcen lässt sich grundsätzlich nichts ändern. Was sich aber ändern lässt, ist die Effizienz der bestehenden Triangulationsschritte. Konkret rate ich für zukünftige Forschung dezidiert von manueller Inhaltsanalyse ab - schlicht, weil der Arbeitsaufwand enorm ist. Obschon eine manuelle Inhaltsanalyse im Sinne des Sinnverstehens immer noch das theoretische Ideal darstellt, existieren mittlerweile zahlreiche automatisierte inhaltsanalytische Verfahren, allen voran Verfahren, welche auf Maschinenlernen basieren (Jordan und T. M. Mitchell 2015), mit denen Texte inhaltlich bereits sehr befriedigend, also für das menschliche Verständnis sinnhaft, analysiert werden können.

\subsubsection{Wessen Agent? Präferenzen und Lobbying}

In der vorliegenden Arbeit habe ich den konzeptionellen Fokus auf die Akteursgruppen der Parlamentarierinnen und Parlamentarier sowie der Journalistinnen und Journalisten gelegt mit der Frage, wie diese Akteursgruppen handeln und, warum sie handeln, wie sie handeln. Im Zuge der teilstrukturierten Leitfadeninterviews haben einige der Interviewpartnerinnen und -partner, sowohl auf Seiten des Parlamentes wie auch der Medien, eine weitere Akteursgruppe bzw. ein zusätzliches Phänomen erwähnt, welches in Kontext dieser Fragestellungen, so die Interviewpartnerinnen und Partner, von Bedeutung sei: Lobbying. Ein Mitglied des Nationalrates etwa beschreibt bereits die physische Präsenz von Lobbyisten in der Wandelhalle des Nationalrates als viel ausgeprägter als die Präsenz der Journalistinnen und Journalisten:

Also ich meine das eine ist die Anwesenheit, die physische Anwesenheit in der Wandelhalle. Aber die gehen quasi unter im Gegensatz zu den Lobbyisten, die ebenfalls anwesend sind.

Dasselbe Mitglied des Nationalrates beschreibt im späteren Gesprächsverlauf, dass diese Lobbyisten nicht bloss Interessenvertreter sind, sondern zunehmend «Public Affairs»-Büros, welche in Auftrag von Interessenvertretern lobbyieren:

Ich stelle andere Dinge fest, wie zum Beispiel, dass wir einen enormen Zuwachs an Public Affairs-Büros haben. Das lässt sich ganz klar feststellen. Da haben wir einen enormen Zuwachs. Die beraten zunehmend Firmen. Es sind nicht mehr Verbände, 
die lobbyieren, sondern es sind viel mehr Public Affairs-Büros, die direkt für eine Firma sehr professionelle Lobbyarbeit machen. Das stellt man fest, also stelle ich fest innerhalb der letzten zweieinhalb Jahre.

Lobbygruppen sind nicht nur bei der inhaltlichen parlamentarischen Arbeit aktiv, sondern teilweise auch im Kontext der Parlamentsberichterstattung, wie ein Mitglied des Ständerates erklärt:

Es gibt Lobbyisten, die mit Medien zusammenarbeiten - ganz klar. Die dann, wenn sie jemanden für ein Thema gewinnen wollen, dann auch gerade noch das Interview organisieren - oder? Oder die Möglichkeit sich zu äussern. Das gibt es, ja.

Ein Journalist beschreibt, dass Lobbygruppen insbesondere bei eingereichten Vorstössen intensiv mitwirken:
Aber sie sind heute derart professionell, dass ein solcher Vorstoss jetzt vielleicht eingereicht wird, und danach, ich nenn jetzt zum Beispiel, der TCS oder der ACS oder ein Verkehrsclub steht noch dahinter, macht noch eine Begleitdokumentation dazu. Die Leute sind ja heute alle ungeheuer vernetzt und haben ja auch ihre Netzwerke. All die Vorstösse rund um die Zweitwohnungen, die schreiben ja in der Regel die Parlamentarier vielleicht schon selber, aber die Dokumentationen, die liefern ihnen beispielsweise, weiss auch nicht, der Fremdenverkehrsverband oder die schweizerische Arbeitsgemeinschaft der Bergführer. All diese Lobby-Gruppen, die dahinter stehen, die irgendein Anliegen haben, sich für etwas einsetzen.

Eine sehr ähnliche Situation beschreibt ein weiterer Journalist und bemüht sich, den Einfluss der Lobbygruppen auch positiv zu deuten:

[A]lso, die schreiben denen ja, ich weiss es jetzt nur von Economiesuisse, die bei verschiedenen bürgerlichen Parteien dann ganze Vorstösse vorschreiben, und man spricht sich sicher zumindest ab, wie man etwas kommuniziert, ob man es gemeinsam macht oder nicht. Aber das machen die Gewerkschaften und die linken Parteien genau gleich, oder, die linken Verkehrsclubs. Das ist ja zum Teil personell auch, sitzen da ja in, also, im VCS sitzen SP-ler und Grüne im Vorstand und so, und drum befruchtet sich das, wenn man es positiv ausdrücken will, befruchtet sich das ja gegenseitig. 
Eine Journalistin sieht Lobbyismus in einer ähnlichen Lesart nicht als Problem, sondern eher als normalen Teil des parlamentarischen Geschehens:

Jeder Parlamentarier ist ein Lobbyist. Er wurde gewählt, damit er für seine Wählerschaft lobbyiert, für die Interessen seiner Wählerschaft; für jene, die am Schluss ihre Stimme für ihn eingelegt haben. Und er ist auch Lobbyist für ganz andere. Sie haben Interessen verschiedenster Art, die sie dann vertreten, erhalten Mandate und lobbyieren dann auch für die. Das finde ich absolut ok.

Diese letzte Meinung wirft die wichtige Frage auf, wie genau Lobbyismus im Kontext der Prinzipal-Agent-Beziehung zwischen Bürgerinnen und Bürgern und Parlamentarierinnen und Parlamentariern aufzufassen ist. Ist Lobbyismus grundsätzlich normativ unbedenklich, weil es sich dabei schlicht um eine Folge des korporatistischen Schweizer Systems (Schmitter 1974) handelt? Oder ist Lobbyismus grundsätzlich normativ problematisch, weil damit eine Form der Einflussnahme stattfindet, welche den Wählerinnen und Wählern vielleicht nicht prinzipiell (die Mitglieder des National- wie auch des Ständerates deklarieren ihre Interessenbindungen im Parlament, allerdings, logischerweise, erst nach der Wahl), aber auf der Ebene der Einzelfälle verborgen bleibt? Es ist nicht ratsam, Lobbygruppen schon allein dadurch, dass es sie gibt, als normativ wünschenswert oder normativ problematisch einzustufen. Die normative Beurteilung von Lobbygruppen muss anhand eines definierbaren und nicht-willkürlichen Kriteriums geschehen. Und ein solches schlage ich mit der vorliegenden Arbeit vor: Der Einfluss von Lobbygruppen im Parlament ist dann normativ unproblematisch, wenn zwischen den Präferenzen der Lobbygruppen, der Präferenzen der Parlamentarierinnen und Parlamentarier, ggf. der Medien, sowie den Präferenzen der Bürgerinnen und Bürger eine Schnittmenge besteht. Handkehrum ist der Einfluss dann normativ problematisch, wenn eine Schnittmenge der Präferenzen nur zwischen Lobbygruppen, Parlamentarierinnen und Parlamentarier sowie ggf. der Medien besteht, unter Ausschluss die Präferenzen der Bürgerinnen und Bürger.

In Abbildung 3 visualisiere ich die Schnittmengen für die drei Präferenzfamilien der Bürgerinnen und Bürger, der Parlamentarierinnen und Parlamentarier sowie der Journalistinnen und Journalisten. Dieses Modell ergänzt um die Präferenzfamilie der Lobbygruppen ist in Abbildung 8 visualisiert. Lobbyismus kann also als weitere Präferenzfamilie in ein normatives Prinzipal-Agent-Modell integriert und damit empirischen Untersuchungen zugänglich gemacht werden. Ist ein solches Vorgehen aber überhaupt sinnvoll? Das Forschungsinteresse der vorliegenden Arbeit liegt ganz bewusst auf den Präferenzfamilien der Politiklogiken und der Medienlogiken. Die Prinzipal-Agent-Beziehung zwischen Bürgerinnen und Bürgern und 
Abbildung 8: Schematische Darstellung der Präferenz-Schnittmengen der Wählerschaft, der Parlamentarierinnen und Parlamentarier, der Medien sowie der Lobbygruppen.

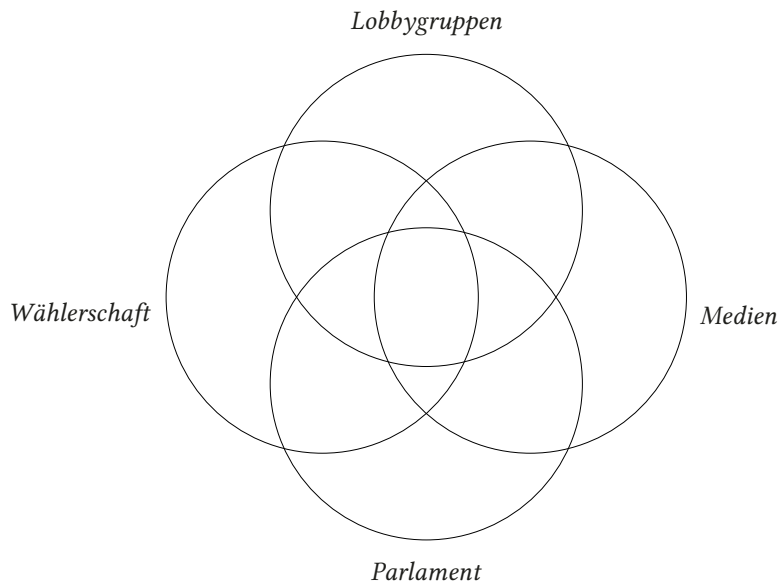

Quelle: Eigene Darstellung.

Parlamentarierinnen und Parlamentariern kann dann und nur dann Bestand haben, wenn eine öffentliche Beobachtbarkeit des Agenten gewährleistet ist. Die öffentliche Sichtbarkeit des Parlamentes wird zum grössten Teil durch Medien, also durch Journalistinnen und Journalisten hergestellt. Das bedeutet, dass die Präferenzfamilie der Medienlogiken als Präferenzen der Journalistinnen und Journalisten ganz fundamental Teil der Prinzipal-Agent-Beziehung sind Medien sind die notwendige Bedingung für die kommunikative Legitimität des Parlamentes. Die Präferenzfamilie der Lobbygruppen hingegen stellt nur eine zusätzliche Präferenzfamilie dar, welche zum Prinzipal-Agent-Problem beiträgt. Das bedeutet, dass Lobbygruppen einer der Gründe sein können, warum Parlamentarierinnen und Parlamentarier Präferenzen haben, welche nicht den Präferenzen der Bürgerinnen und Bürger entsprechen, warum sie also nicht nach Politiklogiken handeln. Die Präferenzfamilie der Lobbygruppen ist aber nur eine von potenziell unendlich vielen zusätzlichen Präferenzfamilien. Eine direkte und unbedingte Verbindung zu der fundamentalen Prinzipal-Agent-Beziehung zwischen Bürgerinnen und Bürgern und Parlamentarierinnen und Parlamentariern hat allerdings ausschliesslich die Präferenzfamilie der Medienlogiken. 
Aus einer rein theoretischen Perspektive ergibt sich somit kein Entdeckungszusammenhang, welcher dem Einfluss von Lobbygruppen besondere Bedeutung zuschreibt. Hier ist aber einzuwenden, dass im Falle der Lobbygruppen zwar nicht die theoretische Herleitung eine empirische Untersuchung rechtfertigt, dafür aber möglicherweise die vermutete empirische Bedeutung der Lobbygruppen. Sollte das Ausmass des Lobbying in der Bundesversammlung tatsächlich so gross sein, wie in den obigen Interviewauszügen angedeutet, dann könnte dies ein dermassen grosses Problem im Sinne der Prinzipal-Agent-Logik darstellen, dass sich eine neue normative Frage stellt: Ab wann ist die Diskrepanz zwischen den Präferenzen der Bürgerinnen und Bürger auf der einen Seite sowie der Schnittmenge der Präferenzen der Parlamentarierinnen und Parlamentarier und der Lobbygruppen auf der anderen Seite derart gross, dass es sich dabei nicht primär um ein theoretisch herleitbares, sondern um ein des empirischen Impacts wegen bedeutendes Problem handelt?

Es ist so gut wie sicher und im Allgemeinen unbestritten, dass Lobby-Aktivitäten spürbaren, bisweilen sehr grossen Einfluss auf Gesetzgebung haben (vgl. Baumgartner, Berry u. a. (2009), Bennedsen und E. Feldmann (2002) und Helpman und Persson (2001)). Das bedeutet allerdings nicht, dass Lobbying aus theoretischer Sicht automatisch normativen Vorrang vor anderen Fragestellungen hat. Der Einfluss von Lobbying ist darum am ehesten als eine Fragestellung rund um die Präferenzgenese von Parlamentarierinnen und Parlamentariern anzusehen, welche in Ergänzung zu anderen normativ und empirisch bedeutsamen Fragestellungen rund um parlamentarisches Handeln beforschenswert ist.

\subsection{Praxisrelevanz der Ergebnisse}

In Unterunterabschnitt 2.1.1 argumentiere ich, dass und warum sozialwissenschaftliche Forschungsvorhaben erst dann Sinn machen, wenn der Grund für das Forschungsvorhaben, der sogannente Entdeckungszusammenhang, hergeleitet wird. Das blosse Reduzieren der Abwesenheit von Information genügt als Begründung für sozialwissenschaftliche Forschung nicht. Dieser Anspruch an explizite Legitimierung sozialwissenschaftlicher Forschung erschöpft sich aber nicht im Entdeckungszusammenhang - auch die Ergebnisse sozialwissenschaftlicher Forschung können nicht kontextfrei als blosser und purer Erkenntnisgewinn, also als das blosse Reduzieren der Abwesenheit von Information, verstanden werden.

Der Umstand, dass sozialwissenschaftliche Forschung stets einem Entdeckungszusammenhang entspringt, bedeutet folglich auch, dass das, was diese Forschung herstellt, im Lichte dieses Entdeckungszusammenhanges interpretiert werden muss. Der Entdeckungszusammenhang meint die normativen und empirischen Umstände, welche Anlass zur Forschung geben, und 
die Ergebnisse der Forschung müssen entsprechend wiederum auf diese normativen und empirischen Umstände zurückgebunden werden. Das bedeutet in der Konsequenz, dass eigentlich jede sozialwissenschaftliche Forschung problemorientierte Forschung ist. Entsprechend müssen die Ergebnisse sozialwissenschaftlicher Forschung auch als Reduktion von Unsicherheit im Handlungsspektrum von Akteuren gedacht werden. Oder, anders ausgedrückt: Sozialwissenschaft hilft Akteuren einerseits, die Wahrscheinlichkeit der empirischen Konsequenzen unterschiedlicher Handlungsoptionen einzuschätzen ${ }^{42}$, und Sozialwissenschaft hilft Akteuren andererseits, die normative Plausibilität unterschiedlicher Handlungsoptionen einzuschätzen.

Sozialwissenschaftliche Forschung, welche diese doppelte Hilfestellung ablehnt und keinerlei Ambition hat, Impact in der in dieser Forschung untersuchten sozialen Realität zu haben, ist recht eigentlich widersprüchlich: Wenn ein bestimmter Aspekt der sozialen Realität im Kontext des Entdeckungszusammenhanges eines Forschungsvorhabens als verhältnismässig wichtiger als alle anderen Aspekte der sozialen Realität angesehen wird ${ }^{43}$, dann drängt sich auch die Frage der Praxisrelevanz auf - wenn ein Aspekt der sozialen Realität im Kontext des Entdeckungszusammenhanges zwar gegenüber allen anderen Aspekten priorisiert wird, aber gleichzeitig bestritten wird, dass die Ergebnisse der Forschung zu diesem einen Aspekt für die Akteure, welche unmittelbar von diesem einen Aspekt betroffen sind, als Hilfestellung relevant sind, dann findet ein offenkundiger Widerspruch statt ${ }^{44}$.

Die Idee, dass sozialwissenschaftliche Forschung letztlich das Ziel haben soll, als Grundlage für Veränderungen der sozialen Realität zu dienen, ist nicht neu. Eine prominente Denkrichtung mit dem Ziel, sozialwissenschaftliche Forschung dezidiert problem- und praxisorientiert durchzuführen, ist die Tradition der «Action Research», zu Deutsch Aktionsforschung. E. T. Stringer (2013, S. 1) beschreibt Aktionsforschung als einen Typus der Forschung, welcher nicht isoliert und abstrakt einzelne Variablen analysiert, sondern sich der Komplexität der Lebenswelt stellt:

Action research is a systematic approach to investigation that enables people to find effective solutions to problems they confront in their everyday lives. Unlike experimental or quantitative research that looks for generalizable explanations

\footnotetext{
42 Auch Nicht-Handeln ist in diesem Sinne eine Handlungsoption mit empirischen Konsequenzen.

43 Diese Priorisierung findet implizit nur schon dadurch statt, dass eben dieser eine Aspekt, und nicht ein beliebiger anderer Aspekt, beforscht wird.

44 Dieses gesamte Argument ist natürlich prinzipieller, idealisierter Art. In der sozialwissenschaftlichen Praxis sind die Motivstrukturen ganz unterschiedlicher Natur, und nur ein Teil der Forschungsvorhaben dürfte komplett offenen Überlegungen rund um Entdeckungszusammenhang und Praxisrelevanz entspringen. Das Spektrum dessen, was beforscht wird, und die Art und Weise, wie dieses beforscht wird, ist zu nicht unwesentlichen Teilen die Folge struktureller und subjektiv-emotionaler (Fehl-)Anreize, wie etwa der «Publish-orPerish»-Dynamiken (H. P. v. Dalen und Henkens 2012; Fanelli 2010; Rond und A. N. Miller 2005).
} 
related to a small number of variables, action research seeks to engage the complex dynamics involved in any social context.

Aktionsforschung zeichnet sich in forschungsprogrammatischer Hinsicht durch die iterative Natur des Forschungsprozesses aus (Avison u. a. 1999): Forschende und Betroffene tauschen sich aus, damit die so entstehenden Feedback-Zyklen zur Problemlösung beitragen. Dieser stete Austausch zwischen Wissenschaft und Praxis ist gleichzeitig auch der normative Grundpfeiler der Aktionsforschung (Brydon-Miller, Greenwood und Maguire 2003). Bisweilen wird Aktionsforschung nach zwei Kategorien differenziert, «Action Research» sowie «Participatory Action Research» (Argyris und Schön 1989), zu Deutsch soviel wie partizipative Aktionsforschung. In dieser Aufteilung soll Participatory Action Research den Austausch mit der Praxis während des Forschungsprozesses stärker betonen, während die blosse Action Research eher darauf abzielt, die Forschungsergebnisse einer Problemlösung zuzuführen, ohne aber die von der Forschung Betroffenen im Laufe des Forschungsprozesses in diesen einzubinden.

Die vorliegende Arbeit ordnet sich nicht in die Tradition der Aktionsforschung ein. Weder wurden die in der Arbeit betroffenen Akteure kontinuierlich in den Forschungsprozess eingebunden (Merkmal der partizipativen Aktionsforschung), noch war das Ziel der Arbeit $a$ priori, einen bestimmten Wandel in der sozialen Realität herbeizuführen (generelles Anliegen der Aktionsforschung). Die Frage der Praxisrelevanz stellt sich für die vorliegende Arbeit nicht aufgrund der im Vorfeld formulierten Absicht, die Ergebnisse praktisch umzusetzen. Die Frage der Praxisrelevanz ist eher, wie oben argumentiert, eine logische Folge des Entdeckungszusammenhanges - dadurch, dass das, was ich in dieser Arbeit beforsche, aus normativen und empirischen Gründen erforschenswert ist, wird das, das diese Arbeit zutage fördert, für die von dieser Arbeit betroffenen Akteure relevant.

Eine Diskussion der auf diese Art verstandenen Praxisrelevanz besteht aus fünf Schritten:

1. Identifikation der betroffenen Stakeholder.

2. Erkenntnisse für die betroffenen Stakeholder.

3. Ausformulieren der Handlungsempfehlungen.

4. Implementierung der Handlungsempfehlungen.

5. Monitoring und Evaluation.

In der vorliegenden Arbeit können, realistischerweise, nur die ersten drei Punkte ausformuliert werden. Konkrete Massnahmen zur Implementierung der Handlungsempfehlungen sowie ein Monitoring und eine Evaluation das Standes der Umsetzung können an dieser Stelle, nachvollziehbarerweise, nicht durchgeführt werden. Allerdings können die ersten drei Punkte 
in einem solchen Masse plausibel hergeleitet werden, dass eine konkrete Implementierung, inklusive Monitoring-Massnahmen, zumindest konzeptuell skizziert werden kann.

\subsubsection{Analyseschritt 1: Identifikation der betroffenen Stakeholder}

Der Begriff der «Stakeholder» ist in erster Linie ein Leihwort aus der Management-Literatur (Brugha und Varvasovszky 2000; Donaldson und Preston 1995) und meint im Grunde Akteure oder Organisationen, welche ein Interesse am Handeln anderer Akteure oder Organisationen haben, weil sie dadurch betroffen sind. Ein Denken im Sinne der Stakeholder-Perspektive ist sinnvoll, weil damit das Spektrum der Akteure, vor allem aber die Art und Weise, wie die Akteure im jeweils interessierenden Kontext von Bedeutung sind, erweitert wird. Es ist denn auch nicht ganz verkehrt, die gesamte vorliegende Arbeit als eine bestimmte Form der Stakeholder-Analyse zu beschreiben. Der Ausgangspunkt der Arbeit, also der Entdeckungszusammenhang, ist gerade, dass das Parlament in einer kommunikativ sehr intimen Beziehung zu den Bürgerinnen und Bürgern, also zum Souverän als Prinzipal steht. In einer solchen Konfiguration sind die Bürgerinnen und Bürger als Souverän denn auch nichts anderes als ein Stakeholder im Kontext des parlamentarischen Handelns: Das, was im Parlament geschieht, betrifft Bürgerinnen und Bürger direkt und unmittelbar auf eine normativ gehaltvolle Art der normative Witz der vorliegenden Arbeit ist in diesem Sinne denn auch, dass Bürgerinnen und Bürger ununterbrochen die Rolle der Stakeholder einnehmen und nicht bloss periodisch in Phasen der Wahlen.

Es ist also recht eigentlich klar, dass Bürgerinnen und Bürger einen Stakeholder im Kontext der vorliegenden Problemstellung darstellen. Sie sind aber nicht die einzigen Stakeholder: Auch Parlamentarierinnen und Parlamentarier bilden eine weitere Stakeholder-Gruppen. Parlamentarierinnen und Parlamentarier sind jene Akteure, welche parlamentarisches Handeln herstellen; das Parlament besteht letztlich aus nichts anderem als aus Parlamentarierinnen und Parlmentariern. Wenn das parlamentarische Personal aber gleichzeitig als Stakeholder $\mathrm{zu}$ verstehen ist, ist zunächst und offensichtlich die Gefahr der konzeptuellen Zirkularität gegeben. Als Stakeholder gelten nämlich Akteure, welche durch das Handeln anderer Akteure beeinflusst sind. Wenn Parlamentarierinnen und Parlamentarier einerseits den Agens darstellen, gleichzeitig aber als Betroffene des Agens zu verstehen sind, macht dies keinen unmittelbaren Sinn. Die Rolle der Parlamenarierinnen und Parlamentarier als Stakeholder ergibt sich erst, wenn parlamentarisches Handeln als Summe individuellen Mikro-Handelns unterschieden wird von parlamentarischem Handeln als globaler Motivstruktur auf Meso-Ebene. Die vorliegende Arbeit hat das Ziel verfolgt, parlamentarisches Handeln in der letzteren Lesart auf Meso-Ebene 
zu verstehen, und zwar im Sinne eines Aggregates individuellen Handelns. Das bedeutet, dass das analytische Ergebnis der empirischen Untersuchung die Präferenzen im Sinne der Präferenzfamilien für keine einzige Person im Parlament wirklich abbildet. Stattdessen zeichnen die Ergebnisse ein globales Bild auf Meso-Ebene. Eine solche konzeptuelle Unterscheidung nach Präferenzen auf Mikro- und Meso-Ebene (bzw., je nach Fragestellung, Makro-Ebene) ist vor allem im Kontext der Wahlforschung gängig und wohl am besten im Konzept des «MedianWählers» umgesetzt. Der Median-Wähler existiert als konkrete Person auf der Mikro-Ebene fast sicher fast immer nicht, sondern lediglich als globales Präferenzaggregat ${ }^{45}$.

\subsubsection{Analyseschritt 2: Erkenntnisse für die betroffenen Stakeholder}

Die Schlussfolgerungen sind nicht für beide Stakeholder-Gruppen gleichermassen umfassend bzw. folgenreich. Für das Parlament auf Mikro-Ebene sind die Schlussfolgerungen besonders aus normativer Hinsicht weniger schwerwiegend als die Schlussfolgerungen für den Stakeholder der Bürgerinnen und Bürger.

Für den Stakeholder des Parlamentes auf Mikro-Ebene, also für individuelle Parlamentarierinnen und Parlamentarier, besteht die erste relevante Erkenntnis in der generellen Skizze der globalen Präferenzen auf Meso-Ebene. Individuelle Parlamentarierinnen und Parlamentarier können in doppelter Hinsicht die globalen Präferenzstrukturen des Parlamentes erfassen. Erstens sind sie, offensichtlicherweise, individuelle Akteure, welche das Parlamentsgeschehen in erster Linie anekdotisch wahrnehmen. In diesem Sinne stellt eine Untersuchung wie die vorliegende die Möglichkeit dar, die Eigenwahrnehmung durch eine systematisierte Fremdwahrnehmung zu ergänzen. Zweitens unterscheidet sich die Art und Weise, wie Parlamentarierinnen und Parlamentarier über ihre Lebenswelt des Parlamentes denken, stark von der Art und Weise, wie in der vorliegenden Untersuchung über das Parlament gedacht wird. Parlamentarisches Handeln verstanden als Handeln im Lichte einer Prinzipal-Agent-Beziehung, fokussiert auf die Frage der Präferenzen, beschreibt das Parlament als ein ein Stück weit zwar abstraktes, dafür aber komplexitätsreduziertes - und somit analysierbares - Modell. Dieses Modell entspricht nicht der parlamentarischen Lebenswelt, aber es ist nicht in einem solchen Masse davon entfernt, dass es für das parlamentarische Personal nicht nachvollziehbar wäre.

Eine zweite relevante Erkenntnis für individuelle Parlamentarierinnen und Parlamentarier besteht in der Quantifizierung des Verhältnisses zwischen Politiklogiken und Medienlogiken. Es ist normativ grundsätzlich wünschenswert, dass Politiklogiken im Parlamentsgeschehen Vor\begin{tabular}{l}
\hline 55 Es sei aber darauf hingewiesen, dass Modell des Median-Wählers seit Langem durchaus umstritten ist (Hinich \\
1977; Romer und Rosenthal 1979).
\end{tabular} 
rang vor Medienlogiken haben. Das bedeutet verallgemeinert, wie in Unterunterabschnitt 2.2.2 argumentiert, dass parlamentarisches Handeln Folge aufrichtiger normativer politischer Überzeugungen sein soll. Wenn Parlamentarierinnen und Parlamentarier in dieser normativen Idealsituation medialisiert, also zugunsten der Präferenzfamilie der Medienlogiken handeln, dann nur als Mittel zum Zweck, um den Politiklogiken stärker zum Ausdruck zu verhelfen. Die empirischen Ergebnisse legen den Schluss nahe, dass beide Bedingungen dieser normativen Idealsituation deutlich nicht erfüllt sind. Weder handeln Parlamentarierinnen und Parlamentarier ausschliesslich nach Politiklogiken (die Wahrscheinlichkeit, dass dem so ist, beträgt lediglich rund 22\%), noch handeln sie medialisiert ausschliesslich als Mittel zum Zweck, um damit auf Politiklogiken zuzuarbeiten (die Wahrscheinlichkeit hierfür beträgt gerade Mal knapp 10\%).

Für den Stakeholder der Bürgerinnen und Bürger sind, zunächst, dieselben Erkenntnisee wie für den Stakeholder des Parlamentes von Bedeutung: Bürgerinnen und Bürger stehen zum Parlament in einer engen, symbiotischen Beziehung, sodass jene Dinge, welche für das Parlament von Bedeutung sind, auch automatisch für Bürgerinnen und Bürger bedeutend werden. Somit ergibt sich zunächst ein globales Bild der Präferenzlagen im Parlament auf der Meso-Ebene. Bürgerinnen und Bürger haben wohl, grundsätzlich, Annahmen über die Präferenzlagen der spezifischen Parlamentarierinnen und Parlamentarier, welche sie wählen (rationaliter sollte genau dies die Motivation zum Wählen sein), aber ein globales Bild der Präferenzlagen ist eine genuin neue Erkenntnis.

Die zweite für Bürgerinnen und Bürger relevante Erkenntnis ist, analog zu jener für den Stakeholder des Parlamentes, die Quantifizierung des Verhältnisses von Politik- zu Medienlogiken im Parlament. Diese Erkenntnis ist für Bürgerinnen und Bürger bedeutender als für Parlamentarierinnen und Parlamentarier, denn, in der Logik der Prinzipal-Agent-Beziehung gedacht, ist es für Bürgerinnen un Bürger als Prinzipal von unmittelbarem Interesse, wie die Präferenzlage des Agenten aussieht. Es ist ja gerade diese Informationsasymmetrie, welche den Kern der Prinzipal-Agent-Beziehung ausmacht: Der Prizipal weiss nicht, in welchem Masse die Präferenzen des Agenten seinen eigenen Präferenzen entsprechen. Die Bedeutung dieser Erkenntnis wird allerdings durch den Umstand abgeschwächt, dass die vorliegende Arbeit ein globales Präferenzbild zeichnet, nicht aber konkrete Motivstrukturen auf Ebene von Parteien oder individuellen Parlamentarierinnen und Parlamentariern; Letzteres wäre für Bürgerinnen und Bürger am interessantesten.

Eine dritte für den Stakeholder der Bürgerinnen und Bürger wichtige Erkenntnis betrifft die Rolle der Medien. Medien sind eine unentbehrliche Komponente der kommunikativen Legitimität des Parlamentes: Erst dadurch, dass Medien über das Parlamentsgeschehen berichten, 
wird das Parlament für Bürgerinnen und Bürger beobachtbar. Die Ergebnisse der vorliegenden Untersuchung legen aber den Schluss nahe, dass Medien nicht einfach passive Berichterstatter sind, sondern die Berichterstattung aktiv und vor allem nach eigenen Präferenzen gestalten. Die Wahrscheinlichkeit, dass Journalistinnen und Journalisten stärker nach reinen Medienlogiken handeln als nach der Schnittmenge von Medienlogiken und Politiklogiken, beträgt rund 55\%. Das ist aus Sicht der Bürgerinnen und Bürger nicht unproblematisch, denn für sie wäre es normativ wünschenswert, dass Medien stärker, wenn nicht gar vorwiegend, ihr Handeln nach der Präferenzfamilie der Politiklogiken ausrichten.

Die vierte für Bürgerinnen und Bürger bedeutende Erkenntnis ist der Befund, dass die Medienkrise im Kontext der Wechselwirkungen zwischen Parlament und Medien mit einiger Wahrschenlichkeit einen Einfluss hat. Wenn auch die inhaltsanalytischen Befunde keine Effekte der Medienkrise nahelegen, werden diese sowohl von Parlamentarierinnen und Parlamentarier wie auch von Journalistinnen und Journalisten deutlich wahrgenommen. Der womöglich wichtigste Effekt der Medienkrise lässt sich unter dem Schlagwort Qualitätseinbussen zusammenfassen. Wenn auch die Berichterstattungsvolumina nicht abnehmen, wandelt sich die Güte der Berichterstattung. Medien, also Journalistinnen und Journalisten, haben weniger Ressourcen zur Verfügung und stehen unter höherem Druck. Zudem findet ein demografischer Wandel auf den Redaktionen statt, im Zuge dessen die Tiefe der Dossier- und Themenkenntnisse erodiert. Diese Veränderungen haben einen direkten Bezug zur Frage der kommunikativen Legitimität, denn die durch Medien hergestellte Beobachtbarkeit des Parlamentes besteht nicht nur aus einer quantitativen Komponente (wie viel wird berichtet), sondern zusätzlich auch aus einer qualitativen Komponente (wie wird berichtet).

\subsubsection{Analyseschritt 3: Ausformulieren der Handlungsempfehlungen}

In Betreff der möglichen Handlungsempfehlungen unterscheiden sich die zwei StakeholderGruppen mit einiger Deutlichkeit voneinander. Während für die Stakeholder-Gruppe der Bürgerinnen und Bürger gewisse verallgemeinerte Handlungsempfehlungen aus den für diese relevanten Befunden ableitbar sind, ist dies für die Stakeholder-Gruppe der Parlamentarierinnen und Parlamentarier kaum möglich. Parlamentarierinnen und Parlamentarier sind autonome Akteure, welche im Rahmen ihrer verfassungsrechtlichen Handlungskompetenz handeln dürfen, wie sie wollen. Nun ist es durchaus möglich, dass Teile dieses Handelns aus einer normativen Perspektive problematisch erscheinen, wie dies eben in der vorliegenden Arbeit der Fall ist. Aus einer naiv-utilitaristischen nromativen Perspektive könnte man nun schlicht das Ziel setzen, dass die Menge an normativ unerwünschtem Handeln zu minimieren sei, bzw., dass die Menge 
an normativ erwünschtem Handeln zu maximieren sei. Ein solches Vorgehen wäre aber sowohl selber normativ problematisch, und es wäre zudem praktischerweise kaum umsetzbar.

Aus normativer Sicht wäre es aus mindestens drei Gründen heikel, das parlamentarische Handeln in jene Richtung zu steuern, wie sie im Rahmen der vorliegenden Arbei normativ wünschenswert wäre. Erstens ist die normative Perspektive der vorliegenden Arbeit, so sehr ich auch von ihr überzeugt bin, bei weitem nicht die einzig mögliche normative Betrachtungsweise parlamentarischen Handelns. Das bedeutet also schlicht, dass die vorliegende normative Perspektive und Einschätzung nur eine mögliche Perspektive und Einschätzung darstellt. Aus einer neo-korporatistischen normativen Perspektive etwa dürfte die Beurteilung parlamentarischen Handelns deutlich anders ausfallen. Der zweite Grund, warum eine Beeinflussung parlamentarischen Handelns in die gewünschte Richtung heikel ist, ist die normativ wünschenswerte Handlungsautonomie der Parlamentarierinnen und Parlamentarier. Mitglieder des Parlamentes sind in ihrer verfassungsrechtlichen Rolle als Agenten in ihrem Handeln prinzipiell frei - und der Witz des Prinzipal-Agent-Beziehung zwischen Wählerschaft und Parlamentarierinnen und Parlamentariern besteht gerade darin, dass ein Handeln im Sinne der Politiklogiken ein solches Handeln ist, bei welchem Parlamentarierinnen und Parlamentarier frei nach bestem Wissen und Gewissen im Glauben handeln, dass sie zugunsten ihrer Wählerschaft handeln. Drittens schliesslich ist die normative Einschätzung in der vorliegenden Arbeit an Empirie gebunden, und diese Empirie wiederum ist mit Ungewissheiten verbunden. Wenn auch die empirischen Ergebnisse nicht vollständig ohne Aussagekraft sind, kann nicht der Anspruch erhoben werden, dass sie ein komplettes und komplett fehlerfreies Bild zeichnen. Stattdessen deuten die Ergebnisse eher eine Silhouette an, welche dann normativ gedeutet werden kann. Diese Silhouette aber zum Anstoss zu nehmen, das Handeln der Parlamentarierinnen und Parlamentarier zu beeinflussen, wäre gegeben der Ungewissheiten dieser Silhouette falsch.

Das parlamentarische Handeln beeinflussen und steuern zu wollen, ist darüber hinaus praktischerweise unmöglich: Individuelle Motivstrukturen vorzuschreiben und diese Vorschriften zu kontrollieren, ist kaum umsetzbar - zumindest nicht in einem halbwegs demokratischen System.

Das parlamentarische Handeln kann normativerweise nicht in eine gewünschte Richtung gesteuert werden. Für die Stakeholder-Gruppe der Bürgerinnen und Bürger ist aber eine normativ vertretbare und praktischerweise umsetzbare Handlungsempfehlung ableitbar. Bürgerinnen und Bürger haben in der Prinzipal-Agent-Beziehung mit dem Parlament die Rolle des Prinzipals. Das grundlegende Problem in einer jeden Prinzipal-Agent-Beziehung ist die Informationsasymmetrie zwischen Prinzipal und Agent. Im Kontext der vorliegenden Arbeit hat sich gezeigt, dass 
der Agent, also Parlamentarierinnen und Parlamentarier, sehr wahrscheinlich in einem nichttrivialen Masse nicht nach Politiklogiken, also nicht primär nach bestem Wissen und Gewissen zugunsten der Wählerschaft handelt. Aus Sicht der Wählerschaft, also aus der Sicht des Prinzipals, ist das ein Problem. Die Lösung dieses Problems besteht aber nicht darin, den Agenten, also Parlamentarierinnen und Parlamentarier, dazu zu zwingen, stärker nach den Politiklogiken zu handeln. Die Lösung besteht stattdessen in der Reduktion der Informationsasymmetrien zwischen Prinzipal und Agent. Dies ist aus der Perspektive der Prinzipal-Agent-Theorie eine recht offensichtliche Lösung, denn sie besteht schlicht darin, das Grundproblem der Beziehung zwischen Prinzipal und Agent, die Informationsasymmetrien, anzugehen.

Wie aber können die Informationsasymmetrien zwischen Bürgerinnen und Bürgern und Parlamentarierinnen und Parlamentariern abgebaut werden? Auch hier gilt es, eine Lösung vorzuschlagen, welche mit anderen normativen Prinzipien kompatibel ist. Das bedeutet, dass es a priori normativ nicht akzeptabel ist, die Handlungsautonomie der Parlamentarierinnen und Parlamentarier einzuschränken, auch nicht im «positiven» Sinn: Es ist nicht zulässig, Parlamentarierinnen und Parlamentarier dazu zu zwingen, in einer wie auch immer gearteten Kommunikationsform regelmässig Rechenschaft abzulegen, um damit Informationen für die Bürgerinnen und Bürger herzustellen. Wenn ein Mehr an Informationen notwendig ist, dieses Mehr aber nicht durch das Parlament selber hergestellt wird, dann bleibt ein anderer, offenkundiger Ort, an welchem diese Informationen hergstellt werden können: Journalismus.

Journalistinnen und Journalisten als bedeutende Quelle öffentlicher Kommunikation haben, so das Argument der vorliegenden Arbeit, eine besondere Rolle im Kontext der kommunikativen Legitimität des Parlamentes. Dadurch, dass sie über die Arbeit des Parlamentes berichten, ermöglichen Sie es Bürgerinnen und Bürgern, Parlamentarierinnen und Parlamentarier als Agenten zu beobachten. Wenn nun aus normativer Sicht ein Mehr an Informationen wünschenswert ist, und dieses Mehr an Informationen durch Journalistinnen und Journalisten hergestellt werden soll, dann stellt sich die Frage, wie diese dazu bewegt werden können. Diese Frage stellt sich auf Mikro-Ebene von Journalistinnen und Journalisten wie auch auf Meso-Ebene der Medienorganisationen.

Eine normativ suboptimale Option ist die Variante der «Brechstange»: Medienschaffende und Medienorganisationen werden schlicht dazu gezwungen, mehr Parlamentsberichterstattung zu betreiben. Diese Option ist offenkundigerweise gleichermassen unrealistisch wie nicht wünschenswert. In demokratischen Gesellschaftsordnungen gibt es praktischerweise keine Instrumente, um eine genehme Art der Berichterstattung zu erzwingen, und normativerweise wäre ein solches Steuern der journalistischen Arbeit problematisch. 
Es ist also offensichtlicherweise kein gangbarer Weg, ein Mehr an Parlamentsberichterstattung zu erzwingen. Es ist aber möglich, Anreize zu setzen, damit Journalistinnen und Journalisten und damit Medienorganisationen frewillig mehr über das Parlament berichten. Oder, im konzeptuellen Jargon der vorliegenden Arbeit: Mit den richtigen Anreizen kann Parlamentsberichterstattung einen höheren Rang in der Präferenzordnung von Journalistinnen und Journalisten bzw. von Medienorganisationen einnehmen.

Die Anreize müssten pekuniärer Natur sein: Journalistische Arbeit ist aufwendig und endlich, und sie ist von Opportunitätskosten geprägt - es mag sein, dass Journalistinnen und Journalisten gerne komplett intrinsisch gerne mehr über das Parlament berichten wollen, aber in der Zeit und mit den Ressourcen, wie sie dies bewerkstelligen würden, können sie auch anderen, für sie potenziell nicht minder interessanten Themen nachgehen, und dabei zusätzlich ein finanzielles Einkommen generieren. All dies ist natürlich recht offensichtlich, aber gerade in Zeiten der Medienkrise ist es notwendig, zu betonen, dass journalistische Arbeit Arbeit ist und nicht Freizeitbeschäftigung. Aus diesem Grund müssen die Anreize für mehr Parlamentsberichterstattung finanzielle Anreize sein.

Wenn von finanziellen Anreizen die Rede ist, dann ist die Rede von einer Massnahme der Medienförderung. Konkret müsste die Medienförderungsmassnahme zugunsten eines Mehr an Parlamentsberichterstattung die Form direkter und selektiver Medienförderung (Künzler u. a. 2013, S. 4) annehmen. Das bedeutet, dass eine öffentliche Förderung von Gefässen und Inhalten, welche sich der Parlamentsberichterstattung widmen, einzurichten wäre. Dies widerspricht der gängigen Praxis der Medienförderung in der Schweiz; üblich und in der Medienbranche akzeptiert sind nach wie vor indirekte Massnahmen der Medienförderung, in erster Linie Posttaxenverbilligung für den Versand von Printerzeugnissen, sowie die (an und für sich direkte) Förderung privater Rundfunkanbieter über das sogenannte Gebührensplitting ${ }^{46}$.

Direkte und selektive Medienförderung betrifft in erster Linie private Medienorganisationen. Die grösste Medienorganisation der Schweiz, die Schweizerische Radio- und Fernsehgesellschaft SRG, übt aber einen öffentlichen Auftrag aus uns ist zu diesem Zweck grossteils öffentlich finanziert. Angesichts der Bedeutung der SRG stellt sich die Frage, ob Medienförderung zugunsten von Parlamentsberichterstattung bei privaten Medien zielführend ist, und, ob stattdessen nicht die SRG Programmgefässe schaffen sollte, welche sich dem Parlamentsgeschehen widmen.

Idealerweise gäbe es sowohl bei privaten Medien wie auch bei der SRG mehr Parlamentsberichterstattung. Praktischerweise sind Ressourcen aber limitiert, und beide Formen der

\footnotetext{
$\overline{46}$ Konzessionierte private Radio- und Fernsehanbieter erhalten einen Anteil der für die SRG erhobenen Gebühren.
} 
zusätzlichen Parlamentsberichterstattung können nicht gleichzeitig verfolgt werden. Auf den ersten Blick mag der Eindruck entstehen, dass es bei einem Entweder-Oder einfacher ist, die SRG dazu zu bewegen, mehr Parlamentsberichterstattung herzustellen, statt neue Instrumente der Förderung privater Medienunternehmen einzuführen. Auf den zweiten Blick dürfte aber recht klar sein, dass diese Variante die normativ und praktisch problematischere ist. Die SRG ist als privater Verein organisiert und sie gestaltet das Programm als Umsetzung des politischen Auftrags frei. Das bedeutet, dass es keine Mechanismen gibt, um konkrete Programminhalte von aussen vorzuschreiben. Diese einzuführen, wäre normativ fragwürdig und politisch ungewiss; damit würde der potenziellen politischen Usurpierung der SRG im Sinne des «politics in broadcasting» (M. Kelly 1984) Tür und Tor geöffnet.

Die Einführung finanzieller Medienförderungsmassnahmen zugunsten der Parlamentsberichterstattung bei privaten Medienorganisationen, andererseits, wäre politisch zwar aufwendig, aber grundsätzlich normativ weniger heikel als Eingriffe in die Programmautonomie der SRG. Bei diesem Instrument handelt es sich um das Schaffen eines Anreizes und nicht eines verbindlichen Zwangs. Das bedeutet, dass diese Massnahme der Medienförderung komplett freiwillig entweder angenommen oder ausgeschlagen werden kann.

Zusammengefasst lassen sich also aus der vorliegenden Untersuchung grundsätzlich zwei Handlungsempfehlungen ableiten:

- Es werden finanzielle Anreize für private Medienunternehmen geschaffen, um mehr über das Parlament zu berichten. Diese Anreize haben die Form direkter und selektiver Medienförderung.

- Die SRG wird dazu bewogen, mit den der SRG zur Verfügung stehenden Ressourcen mehr über das Parlament zu berichten.

Von diesen zwei potenziellen Massnahmen ist die erste, die Schaffung von Förderung für private Medienunternehmen, die praktisch realistischere und normativ wünschenswertere.

\subsubsection{Umsetzungsschritt: Implementierung, Monitoring und Evaluation}

Das Ausarbeiten eines umfassenden Umsetzungsplanes ist im Rahmen der vorliegenden Arbeit nicht angebracht. Dies ist nicht einem Mangel an Ideen und Fantasie geschuldet, sondern dem Kontext - ein unilateral erarbeiteter Umsetzungsplan hat keine Aussicht auf Umsetzung. Die Umsetzung wäre eine grosse politische Aufgabe, denn das Konzept der selektiven Förderung von Medieninhalten ist in der gegenwärtigen Medienförderungs-Debatte der Schweiz ein eher heisses Eisen. Am ehesten bietet die Eidgenössische Medienkommission EMEK (2015) 
mit ihren Vorschlägen für die zukünftige Ausgestaltung der Medienförderung in der Schweiz einen möglichen Anknüpfungspunkt. Im Diskussionspapier der EMEK wird vorgeschlagen, eine staatsferne Stiftung einzurichten, welche den Zweck verfolgt, private Medien zu fördern. Die Stossrichtung des EMEK-Vorschlages ist allgemeine direkte und indirekte Förderung über unterschiedliche Massnahmen. Die angedachte Stiftung hätte aber auch die Kompetenz, einzelne Inhalte selektiv und direkt zu fördern (ebd., S. 21):

Die Stiftung wäre in der Lage, Unterstützungsgesuche für Medien-«Infrastrukturen» zu genehmigen sowie Entwicklungs- und Produktionsaufträge zu vergeben.

Mit der Kompetenz ausgestattet, «Entwicklungs- und Produktionsaufträge» zu vergeben, wäre die von der EMEK vorgeschlagene Stiftung ein geeigneter Ort, um Medienförderung zum Zwecke eines Mehr an Parlamentsberichterstattung zu realisieren.

Open Access Dieses Buch wird unter der Creative Commons Namensnennung - Nicht kommerziell 4.0 International Lizenz (http://creativecommons.org/licenses/by-nc/4.0/deed. de) veröffentlicht, welche für nicht kommerzielle Zwecke die Nutzung, Vervielfältigung, Bearbeitung, Verbreitung und Wiedergabe in jeglichem Medium und Format erlaubt, sofern Sie den/die ursprünglichen Autor(en) und die Quelle ordnungsgemäß nennen, einen Link zur Creative Commons Lizenz beifügen und angeben, ob Änderungen vorgenommen wurden.

Etwaige Abbildungen oder sonstiges Drittmaterial unterliegen ebenfalls der genannten Creative Commons Lizenz, sofern sich aus der Abbildungslegende oder der Quellreferenz nichts anderes ergibt. Sofern solches Drittmaterial nicht unter der genannten Creative Commons Lizenz steht, ist eine Vervielfältigung, Bearbeitung oder öffentliche Wiedergabe nur mit vorheriger Zustimmung des betreffenden Rechteinhabers oder auf der Grundlage einschlägiger gesetzlicher Erlaubnisvorschriften zulässig. 


\section{Literatur}

Akobeng, A. K. (2005). "Understanding systematic reviews and meta-analysis". In: Archives of Disease in Childhood 90.8, S. 845-848. Dor: 10.1136/adc.2004.058230 (siehe S. 53).

Altheide, David L. und Robert P. Snow (1979). Media logic. Beverly Hills und Calif: Sage Publications. ISBN: 0-8039-1297-8 (siehe S. 48).

Amsel, Eric, Paul A. Klaczynski, Adam Johnston, Shane Bench, Jason Close, Eric Sadler und Rick Walker (2008). “A dual-process account of the development of scientific reasoning: The nature and development of metacognitive intercession skills". In: Cognitive Development. Scientific reasoning - Where are we now?Sodian and Bullock SI 23.4, S. 452-471. DoI: 10.1016/j.cogdev.2008.09.002 (siehe S. 35).

Argyris, Chris und Donald A. Schön (1989). "Participatory Action Research and Action Science Compared A Commentary”. In: American Behavioral Scientist 32.5, S. 612-623. DoI: 10.1177 /0002764289032005008 (siehe S. 190).

Austin, J. L. (1975). How to do things with words. Cambridge: Harvard University Press. ISBN: 0-674-41152-8 (siehe S. 28).

Avison, David E., Francis Lau, Michael D. Myers und Peter Axel Nielsen (1999). “Action Research”. In: Commun. ACM 42.1, S. 94-97. Dor: 10.1145/291469.291479 (siehe S. 190).

Bafumi, Joseph und Michael C. Herron (2010). "Leapfrog Representation and Extremism: A Study of American Voters and Their Members in Congress". In: American Political Science Review 104.03, S. 519-542. DoI: 10.1017/S0003055410000316 (siehe S. 55, 56).

Baker, C. Edwin (2006). Media Concentration and Democracy: Why Ownership Matters. Cambridge ; New York: Cambridge University Press. IsBn: 978-0-521-68788-1 (siehe S. 44).

Barnett, Steven (2002). "Will a Crisis in Journalism Provoke a Crisis in Democracy?" In: The Political Quarterly 73.4, S. 400-408. DoI: 10.1111/1467-923X.00494 (siehe S. 44).

Baumgartner, Frank R., Jeffrey M. Berry, Marie Hojnacki, David C. Kimball und Beth L. Leech (2009). Lobbying and Policy Change: Who Wins, Who Loses, and Why. Chicago ; London: University Of Chicago Press. ISBN: 978-0-226-03945-9 (siehe S. 188).

Baumgartner, Frank R. und Bryan D. Jones (2010). Agendas and Instability in American Politics, Second Edition. University of Chicago Press. ISBN: 978-0-226-03953-4 (siehe S. 58, 71).

Baumgartner, Frank R., Bryan D. Jones und Peter B. Mortensen (2014). "Punctuated equilibrium theory: Explaining stability and change in public policymaking”. In: Theories of the Policy Process. Hrsg. von Paul A. Sabatier und Christopher Weible. Boulder, Colorado: Westview Press, S. 59-104. ISBN: 978-0-8133-4926-8 (siehe S. 71). 
Beck, Nathaniel (2001). “Time-series-cross-section Data”. In: Statistica Neerlandica 55.2, S. 111133. Dor: 10.1111/1467-9574.00161 (siehe S. 111).

- (2006). Time-Series-Cross-Section Methods (siehe S. 112).

Beck, Nathaniel und Jonathan N. Katz (1995). "What to do (and not to do) with Time-Series Cross-Section Data”. In: The American Political Science Review 89.3, S. 634-647. DOI: 10.2307 /2082979 (siehe S. 112).

- (2011). "Modeling Dynamics in Time-Series-Cross-Section Political Economy Data". In: Annual Review of Political Science 14.1, S. 331-352. DoI: 10.1146/annurev-polisci-071510-103 222 (siehe S. 112).

Bennedsen, Morten und Sven E. Feldmann (2002). “Lobbying Legislatures”. In: Journal of Political Economy 110.4, S. 919-946. DOI: 10.1086/340775 (siehe S. 188).

Bennett, W. Lance (1990). “Toward a Theory of Press-State Relations in the United States". In: Journal of Communication 40.2, S. 103-127. DOI: 10.1111/j.1460-2466.1990.tb02265.x (siehe S. 58).

Bergman, Torbjörn, Wolfgang C. Müller und Kaare Strøm (2000). "Introduction: Parliamentary democracy and the chain of delegation". In: European fournal of Political Research 37.3, S. 255-260. DoI: 10.1111/1475-6765.00512 (siehe S. 39).

Bishin, Benjamin G. (2000). “Constituency Influence in Congress: Does Subconstituency Matter?” In: Legislative Studies Quarterly 25.3, S. 389-415. DoI: 10.2307/440413 (siehe S. 56).

Blom, Jonas Nygaard und Kenneth Reinecke Hansen (2015). "Click bait: Forward-reference as lure in online news headlines”. In: fournal of Pragmatics 76, S. 87-100. DOI: 10.1016 /j.pragma.2014.11.010 (siehe S. 44).

Blum, Sonja (2011). Politikfeldanalyse. VS Verlag für Sozialwissenschaften. IsBN: 978-3-53117276-7 (siehe S. 100).

Blumler, Jay G. und Dennis Kavanagh (1999). "The Third Age of Political Communication: Influences and Features”. In: Political Communication 16.3, S. 209-230 (siehe S. 79, 80).

Boczkowski, Pablo J. und Eugenia Mitchelstein (2015). The News Gap. Cambridge: Mit Press Ltd. ISBN: 978-0-262-52826-9 (siehe S. 61).

Boczkowski, Pablo J. und Limor Peer (2011). "The Choice Gap: The Divergent Online News Preferences of Journalists and Consumers". In: Journal of Communication 61.5, S. 857-876. DOI: 10.1111/j.1460-2466.2011.01582.x (siehe S. 61).

Bonfadelli, Heinz und Thomas N. Friemel (2014). Medienwirkungsforschung. Konstanz und München: UTB / UVK (siehe S. 77). 
Bonfadelli, Heinz, Ursula Schwarb, Werner A. Meier, Irène Perrin, Josef Trappel, Matthias Künzler, Manuel Puppis, Edzard Schade, Andreas Ledergerber und Otfried Jarren (2005). Medienkonzentration in Regionen: Vielfalt, Medienmacht und Regulierung. Zürich: IPMZ (siehe S. 42).

Brandenburg, Heinz (2002). "Who Follows Whom? The Impact of Parties on Media Agenda Formation in the 1997 British General Election Campaign”. In: The Harvard International Journal of Press/Politics 7.3, S. 34-54. DoI: 10.1177/1081180X0200700303 (siehe S. 112).

Brants, Kees und Philip van Praag (2015). "Beyond Media Logic”. In: fournalism Studies, S. 1-14. DOI: 10.1080/1461670X.2015.1065200 (siehe S. 78).

Braun, Virginia und Victoria Clarke (2006). "Using thematic analysis in psychology”. In: Qualitative Research in Psychology 3.2, S. 77-101. Dor: 10.1191/1478088706qp063oa (siehe S. 102). Brooks, Rodney A. (1991). "Intelligence without reason”. In: COMPUTERS AND THOUGHT, IfCAI-91. San Francisco: Morgan Kaufmann, S. 569-595 (siehe S. 45).

Brugha, Ruairí und Zsuzsa Varvasovszky (2000). “Stakeholder analysis: a review”. In: Health Policy and Planning 15.3, S. 239-246. Dor: 10.1093/heapol/15.3.239 (siehe S. 191).

Bryant, Jennings und Dorina Miron (2004). "Theory and Research in Mass Communication”. In: Journal of Communication 54.4, S. 662-704. DoI: 10.1111/j.1460-2466.2004.tb02650.x (siehe S. 69).

Brydon-Miller, Mary, Davydd Greenwood und Patricia Maguire (2003). "Why Action Research?" In: Action Research 1.1, S. 9-28. DOI: 10.1177/14767503030011002 (siehe S. 190).

Buss, David M. (2008). Evolutionary Psychology: The New Science of the Mind. Pearson/Allyn und Bacon. ISBN: 978-0-205-48338-9 (siehe S. 33).

Bütikofer, Sarah (2014). Das Schweizer Parlament: Eine Institution auf dem Pfad der Moderne. Baden-Baden: Nomos. IsBn: 978-3-8487-0817-8 (siehe S. 67).

Bütikofer, Sarah und Simon Hug (2010). “The Swiss Upper House: 'Chambre de Réflexion' or Conservative Renegades?” In: The fournal of Legislative Studies 16.2, S. 176-194. DoI: 10.1080/13572331003740099 (siehe S. 57).

- (2015). "Strategic Behaviour in Parliament". In: The fournal of Legislative Studies 21.3, S. 295322. DOI: 10.1080/13572334.2014.997988 (siehe S. 57).

Camerer, Colin (1998). "Bounded Rationality in Individual Decision Making”. In: Experimental Economics 1.2, S. 163-183. DOI: 10.1023/A:1009944326196 (siehe S. 84).

Cameron, A. Colin und Pravin K. Trivedi (2013). Regression Analysis of Count Data. Cambridge: Cambridge University Press. IsBN: 978-1-107-71779-4 (siehe S. 112). 
Cammarano, Stephanie Schwab (2013). Rollen in der Politikvermittlung: Die Interaktion zwischen Politik und fournalismus in der Schweiz. Baden-Baden: Nomos. ISBN: 978-3-8487-0430-9 (siehe S. 60).

Carlson, Matt (2014). "When news sites go native: Redefining the advertising-editorial divide in response to native advertising”. In: fournalism, S. 1464884914545441. DOI: 10.1177/146488 4914545441 (siehe S. 44).

- (2015). “The Robotic Reporter”. In: Digital fournalism 3.3, S. 416-431. DoI: 10.1080/21670811 .2014.976412 (siehe S. 45).

Carman, Christopher Jan (2006). "Public Preferences for Parliamentary Representation in the UK: An Overlooked Link?” In: Political Studies 54.1, S. 103-122. DoI: 10.1111/j.1467-9248.20 06.00568.x (siehe S. 56).

Chakravartty, Anjan (2001). "The Semantic or Model-Theoretic View of Theories and Scientific Realism”. In: Synthese 127.3, S. 325-345. DoI: 10.1023/A:1010359521312 (siehe S. 72).

Chiou, Lesley und Catherine Tucker (2013). "Paywalls and the demand for news". In: Information Economics and Policy 25.2, S. 61-69. DoI: 10.1016/j.infoecopol.2013.03.001 (siehe S. 41).

Cipriani, Andrea und John Geddes (2003). "Comparison of systematic and narrative reviews: the example of the atypical antipsychotics". In: Epidemiology and Psychiatric Sciences 12.03, S. 146-153. DoI: 10.1017/S1121189X00002918 (siehe S. 53).

Clerwall, Christer (2014). "Enter the Robot Journalist”. In: fournalism Practice 8.5, S. 519-531. DOI: $10.1080 / 17512786.2014 .883116$ (siehe S. 45).

Cohen, Akiba A. (1976). "Radio vs. TV: The Effect of the Medium". In: Journal of Communication 26.2, S. 29-35. DoI: 10.1111/j.1460-2466.1976.tb01376.x (siehe S. 69).

Cohen, Jacob (1990). “Things I have learned (so far)”. In: American Psychologist 45.12, S. 13041312. DoI: 10.1037/0003-066X.45.12.1304 (siehe S. 121).

- (1994). "The earth is round ( < . .05)". In: American Psychologist 49.12, S. 997-1003 (siehe S. 121).

Cole, Stephen (1983). “The Hierarchy of the Sciences?” In: American fournal of Sociology 89.1, S. 111-139 (siehe S. 25).

Collins, John A. und Bart C. J. M. Fauser (2005). "Balancing the strengths of systematic and narrative reviews". In: Human Reproduction Update 11.2, S. 103-104. DoI: 10.1093/humupd/ dmh058 (siehe S. 53).

Cook, Jonathan E. und Shahzeen Z. Attari (2012). "Paying for What Was Free: Lessons from the New York Times Paywall”. In: Cyberpsychology, Behavior, and Social Networking 15.12, S. 682-687. DoI: 10.1089/cyber.2012.0251 (siehe S. 41). 
Cook, Timothy E. (1986). "House Members as Newsmakers: The Effects of Televising Congress". In: Legislative Studies Quarterly 11.2, S. 203-226. Dor: 10.2307/439876 (siehe S. 65).

- (1990). Making Laws and Making News: Media Strategies in the U.S. House of Representatives. Brookings Inst Pr. ISBN: 978-0-8157-1557-3 (siehe S. 58).

Croissant, Yves und Giovanni Millo (2008). "Panel data econometrics in R: The plm package”. In: Journal of Statistical Software 27.2, S. 1-43 (siehe S. 112).

Dahlgren, Peter (2006). "Doing citizenship The cultural origins of civic agency in the public sphere". In: European Journal of Cultural Studies 9.3, S. 267-286. Dor: 10.1177/136754940606 6073 (siehe S. 30).

Dalen, Hendrik P. van und Kène Henkens (2012). "Intended and unintended consequences of a publish-or-perish culture: A worldwide survey”. In: Journal of the American Society for Information Science and Technology 63.7, S. 1282-1293. DoI: 10.1002/asi.22636 (siehe S. 189).

Davison, W. Phillips (1983). "The Third-Person Effect in Communication”. In: Public Opinion Quarterly 47.1, S. 1-15. DoI: 10.1086/268763 (siehe S. 136).

Demers, David Pearce, Dennis Craff, Yang-Ho Choi und Beth M. Pessin (1989). "Issue Obtrusiveness and the Agenda-Setting Effects of National Network News". In: Communication Research 16.6, S. 793-812. Dor: 10.1177/009365089016006004 (siehe S. 99).

Denham, Bryan E. (2010). "Toward Conceptual Consistency in Studies of Agenda-Building Processes: A Scholarly Review”. In: Review of Communication 10.4, S. 306-323. Dor: 10.1080 /15358593.2010.502593 (siehe S. 72).

Denzin, Norman K. (1970). The Research Act: A Theoretical Introduction to Sociological Methods. Transaction Publishers. ISBN: 978-0-202-36859-7 (siehe S. 95).

- (2012). “Triangulation 2.0”. In: Journal of Mixed Methods Research 6.2, S. 80-88. DoI: 10.1177 /1558689812437186 (siehe S. 96).

Dewey, John (1954). The Public and Its Problems. Athens: Swallow Press. ISBN: 978-0-8040-0254-7 (siehe S. 27).

Donaldson, Thomas und Lee E. Preston (1995). “The Stakeholder Theory of the Corporation: Concepts, Evidence, and Implications". In: The Academy of Management Review 20.1, S. 6591. Dor: $10.2307 / 258887$ (siehe S. 191).

Druckman, James N. (2003). "The Power of Television Images: The First Kennedy-Nixon Debate Revisited”. In: Journal of Politics 65.2, S. 559-571. Dor: 10.1111/1468-2508.t01-1-00015 (siehe S. 69).

Economist Intelligence Unit (2015). Democracy Index 2014. Democracy and its discontents. London: The Economist Intelligence Unit (siehe S. 31). 
Edelman, Murray (1985). The symbolic uses of politics. Urbana: University of Illinois Press. ISBN: 978-0-252-01202-0 (siehe S. 58).

Edwards III, George C. und B. Dan Wood (1999). "Who Influences Whom? The President, Congress, and the Media”. In: The American Political Science Review 93.2, S. 327-344. DoI: 10.2307/2585399 (siehe S. 58).

Eidgenössische Medienkommission EMEK (2015). Medienförderung: Standortbestimmung und Empfehlungen für die Zukunft (siehe S. 198, 199).

Elmelund-Præstekær, Christian, David Nicolas Hopmann und Asbjørn Sonne Nørgaard (2011). "Does Mediatization Change MP-Media Interaction and MP Attitudes toward the Media? Evidence from a Longitudinal Study of Danish MPs". In: International fournal of Press/Politics 16.3, S. 382-403 (siehe S. 65).

Entman, Robert M. (1993). "Framing: Toward Clarification of a Fractured Paradigm”. In: fournal of Communication 43.4, S. 51-58 (siehe S. 70).

Erikson, Robert S., Norman R. Luttbeg und William V. Holloway (1975). “Knowing One’s District: How Legislators Predict Referendum Voting”. In: American fournal of Political Science 19.2, S. 231-246. DOI: 10.2307/2110434 (siehe S. 55).

Ettinger, Patrik (2005). "Das Parlament in der politischen Kommunikation der Schweiz". In: Politische Kommunikation in der Schweiz. Hrsg. von Patrick Donges und Otfried Jarren. Bern: Haupt, S. 75-90. ISBN: 978-3-258-06765-0 (siehe S. 40).

- (2013). "Wandel der Politikberichterstattung". In: Krise, Wandel, Aufbruch. Hrsg. von Projektverbund Sinergia "Medienkrise". Universität Zürich: SwissGIS (siehe S. 52, 65).

Evans, Jonathan St. B. T. (2003). "In two minds: dual-process accounts of reasoning". In: Trends in Cognitive Sciences 7.10, S. 454-459. Dor: 10.1016/j.tics.2003.08.012 (siehe S. 34).

Fanelli, Daniele (2010). “Do Pressures to Publish Increase Scientists' Bias? An Empirical Support from US States Data”. In: PLoS ONE 5.4, e10271. DoI: 10.1371/journal.pone.0010271 (siehe S. 189).

Fereday, Jennifer und Eimear Muir-Cochrane (2008). "Demonstrating Rigor Using Thematic Analysis: A Hybrid Approach of Inductive and Deductive Coding and Theme Development". In: International fournal of Qualitative Methods 5.1, S. 80-92 (siehe S. 102).

Ferejohn, John A. (1991). "Rationality and Interpretation: Parliamentary Elections in Early Stuart England”. In: The Economic Approach to Politics: A Critical Reassessment of the Theory of Rational Action. Hrsg. von Kristen Renwick Monroe. New York, NY: HarperCollins Publishers, S. 279-305. IsBN: 978-0-673-46426-2 (siehe S. 85). 
Ferree, Myra Marx (2002). Shaping Abortion Discourse: Democracy and the Public Sphere in Germany and the United States. Cambridge University Press. IsBn: 978-0-521-79384-1 (siehe S. 30).

Feyerabend, Paul (1963). "Materialism and the Mind-Body Problem”. In: The Review of Metaphysics 17.1, S. 49-66 (siehe S. 33).

Fletcher, Logan und Peter Carruthers (2012). "Metacognition and reasoning”. In: Philosophical Transactions of the Royal Society of London B: Biological Sciences 367.1594, S. 1366-1378. DoI: 10.1098/rstb.2011.0413 (siehe S. 35).

Flick, Uwe (2014). An Introduction to Qualitative Research. Los Angeles: SAGE Publications Ltd. ISBN: 978-1-4462-6779-0 (siehe S. 107).

Flick, Uwe, Vjenka Garms-Homolová, Wolfram J. Herrmann, Joachim Kuck und Gundula Röhnsch (2012). ““I Can’t Prescribe Something Just Because Someone Asks for It . . . Using Mixed Methods in the Framework of Triangulation”. In: fournal of Mixed Methods Research 6.2, S. 97-110. DOI: 10.1177/1558689812437183 (siehe S. 96).

fög-Forschungsbereich Öffentlichkeit und Gesellschaft (2014). Fahrbuch 2014 Qualität der Medien: Schweiz - Suisse- Svizzera. Basel: Schwabe. ISBN: 978-3-7965-3320-4 (siehe S. 43).

Frank, Russell (2003). “These Crowded Circumstances': When Pack Journalists Bash Pack Journalism”. In: Journalism 4.4, S. 441-458 (siehe S. 148).

Frankish, Keith (2010). "Dual-Process and Dual-System Theories of Reasoning”. In: Philosophy Compass 5.10, S. 914-926. DoI: 10.1111/j.1747-9991.2010.00330.x (siehe S. 34).

Frantzich, Stephen und John Sullivan (1996). The C-SPAN Revolution. Norman: University of Oklahoma Press. ISBN: 978-0-8061-2870-2 (siehe S. 64).

Gallie, Walter B. (1955). "Essentially Contested Concepts". In: Proceedings of the Aristotelian Society 56, S. 167-198 (siehe S. 30).

Galtung, Johan und Mari Holmboe Ruge (1965). "The Structure of Foreign News The Presentation of the Congo, Cuba and Cyprus Crises in Four Norwegian Newspapers". In: Journal of Peace Research 2.1, S. 64-90. DoI: 10.1177/002234336500200104 (siehe S. 49).

Gavison, Ruth (1992). "Feminism and the Public/Private Distinction". In: Stanford Law Review 45.1, S. 1-45. DOI: 10.2307/1228984 (siehe S. 26).

Gehanno, Jean-François, Laetitia Rollin und Stefan Darmoni (2013). "Is the coverage of google scholar enough to be used alone for systematic reviews". In: BMC Medical Informatics and Decision Making 13.1, S. 7. DoI: 10.1186/1472-6947-13-7 (siehe S. 54).

Gelman, Andrew (2013). "P Values and Statistical Practice”. In: Epidemiology 24.1, S. 69-72. DoI: 10.1097/EDE.0b013e31827886f7 (siehe S. 121). 
Gerhards, Jürgen (1998). "Öffentlichkeit”. In: Politische Kommunikation in der demokratischen Gesellschaft. Hrsg. von Otfried Jarren, Ulrich Sarcinelli und Ulrich Saxer. VS Verlag für Sozialwissenschaften, S. 268-274. ISBN: 978-3-322-80349-8 978-3-322-80348-1 (siehe S. 27).

Gerhards, Jürgen und Friedhelm Neidhardt (1990). Strukturen und Funktionen moderner Öffentlichkeit: Fragestellungen und Ansätze. Techn. Ber. FS III 90-101. WZB Discussion Paper (siehe S. 27).

Gibbs, Martin, James Meese, Michael Arnold, Bjorn Nansen und Marcus Carter (2015). "\#Funeral and Instagram: death, social media, and platform vernacular”. In: Information, Communication \& Society 18.3, S. 255-268. Dor: 10.1080/1369118X.2014.987152 (siehe S. 81).

Giere, Ronald N. (2004). "How Models Are Used to Represent Reality". In: Philosophy of Science 71.5, S. 742-752 (siehe S. 72).

Goffman, Erving und Bennett Berger (1986). Frame Analysis: An Essay on the Organization of Experience. Boston: Northeastern. ISBN: 978-0-930350-91-8 (siehe S. 70).

Golan, Guy und Wayne Wanta (2001). "Second-Level Agenda Setting in the New Hampshire Primary: A Comparison of Coverage in Three Newspapers and Public Perceptions of Candidates". In: fournalism \& Mass Communication Quarterly 78.2, S. 247-259. DoI: 10.1177 /107769900107800203 (siehe S. 69).

Goldstein, Doris S. (1964). “Alexis de Tocqueville’s Concept of Citizenship”. In: Proceedings of the American Philosophical Society 108.1, S. 39-53 (siehe S. 37).

Goode, William J. (1997). "Rational choice theory". In: The American Sociologist 28.2, S. 22-41. DOI: $10.1007 / \mathrm{s} 12108-997-1004-5$ (siehe S. 84).

Graham, Stephen (1998). "The end of geography or the explosion of place? Conceptualizing space, place and information technology". In: Progress in Human Geography 22.2, S. 165-185. DOI: 10.1191/030913298671334137 (siehe S. 29).

Green, Donald und Ian Shapiro (1996). Pathologies of Rational Choice Theory: A Critique of Applications in Political Science. New Haven: Yale University Press. ISBN: 978-0-300-06636-4 (siehe S. 85, 86).

Grossman, Sanford J. und Oliver D. Hart (1983). “An Analysis of the Principal-Agent Problem”. In: Econometrica 51.1, S. 7. DoI: 10.2307/1912246 (siehe S. 39).

Habermas, Jürgen (1976). “Some Distinctions in Universal Pragmatics: A Working Paper”. In: Theory and Society 3.2, S. 155-167 (siehe S. 28).

- (1981). Theorie des kommunikativen Handelns (2 Bände). Frankfurt am Main: Suhrkamp. ISBN: 3-518-28775-3 (siehe S. 28, 34). 
- (1982). Zur Logik der Sozialwissenschaften. Frankfurt am Main: Suhrkamp Verlag. ISBN: 978-3-518-57622-9 (siehe S. 25).

- (1992). Faktizität und Geltung. Frankfurt am Main: Suhrkamp. IsBN: 978-3-518-58126-1 (siehe S. 27).

- (1994). “Three Normative Models of Democracy”. In: Constellations 1.1, S. 1-10. Dor: 10.111 1/j.1467-8675.1994.tb00001.x (siehe S. 38).

- (1997). "Popular sovereignty as procedure”. In: Deliberative Democracy: Essays on Reason and Politics. Hrsg. von James Bohman und William Rehg. Cambridge, Mass: The MIT Press, S. 35-65. ISBN: 978-0-262-52241-0 (siehe S. 38).

- (2008). "Hat die Demokratie noch eine epistemische Dimension?" In: soFid Politische Soziologie 2, S. 9-38 (siehe S. 30).

Habermas, Jürgen, Sara Lennox und Frank Lennox (1974). “The Public Sphere: An Encyclopedia Article (1964)". In: New German Critique 3, S. 49-55. Dor: 10.2307/487737 (siehe S. 30).

Hall, Peter A. und Rosemary C. R. Taylor (1996). "Political Science and the Three New Institutionalisms". In: Political Studies 44.5, S. 936-957 (siehe S. 86).

Hänggli, Regula (2012). "Key Factors in Frame Building: How Strategic Political Actors Shape News Media Coverage”. In: American Behavioral Scientist 56.3, S. 300-317 (siehe S. 63).

Hechter, Michael und Satoshi Kanazawa (1997). "Sociological Rational Choice Theory". In: Annual Review of Sociology 23, S. 191-214 (siehe S. 84, 85).

Helpman, Elhanan und Torsten Persson (2001). "Lobbying and Legislative Bargaining”. In: Advances in Economic Analysis \& Policy 1.1 (siehe S. 188).

Hinich, Melvin J. (1977). "Equilibrium in spatial voting: The median voter result is an artifact". In: Journal of Economic Theory 16.2, S. 208-219. Dor: 10.1016/0022-0531(77)90005-9 (siehe S. 192).

Hjarvard, Stig (2008). "The Mediatization of Society. A Theory of the Media as Agents of Social and Cultural Change.” In: Nordicom Review 29.2, S. 105-134 (siehe S. 74).

Hofstetter, Brigitte und Philomen Schönhagen (2014). "Wandel redaktioneller Strukturen und journalistischen Handelns". In: Studies in Communication / Media 3.2, S. 228-252 (siehe S. 43).

Holcomb III, Harmon R. (1996). "Just so stories and inference to the best explanation in evolutionary psychology”. In: Minds and Machines 6.4, S. 525-540. Dor: 10.1007/BF00389657 (siehe S. 33).

Hölmstrom, Bengt (1979). "Moral Hazard and Observability”. In: The Bell fournal of Economics 10.1, S. 74-91. DOI: 10.2307/3003320 (siehe S. 39). 
Hopf, Christel (2004). “Qualitative Interviews: An Overview”. In: A Companion to Qualitative Research. Hrsg. von Uwe Flick, Ernst von Kardoff und Ines Steinke. London: SAGE, S. 203208. ISBN: 978-0-7619-7374-4 (siehe S. 106, 107).

Hopmann, David Nicolas, Claes H. de Vreese und Erik Albaek (2011). "Incumbency Bonus in Election News Coverage Explained: The Logics of Political Power and the Media Market”. In: Journal of Communication 61.2, S. 264-282 (siehe S. 99).

Horai, S., K. Hayasaka, R. Kondo, K. Tsugane und N. Takahata (1995). "Recent African origin of modern humans revealed by complete sequences of hominoid mitochondrial DNAs." In: Proceedings of the National Academy of Sciences 92.2, S. 532-536. DoI: 10.1073/pnas.92.2.532 (siehe S. 32).

Horwitz, Morton J. (1982). "The History of the Public/Private Distinction”. In: University of Pennsylvania Law Review 130.6, S. 1423-1428. Dor: 10.2307/3311976 (siehe S. 26).

Howard, Don (2006). "Lost Wanderers in the Forest of Knowledge: Some Thoughts on the Discovery-Justification Distinction”. In: Revisiting Discovery and fustification: Historical and Philosophical Perspectives on the Context Distinction. Hrsg. von Jutta Schickore und Friedrich Steinle. Springer Science \& Business Media, S. 3-22. IsBN: 978-1-4020-4250-8 (siehe S. 25).

Howe, Kenneth R. (2012). “Mixed Methods, Triangulation, and Causal Explanation”. In: fournal of Mixed Methods Research 6.2, S. 89-96. DoI: 10.1177/1558689812437187 (siehe S. 96).

Hsieh, Hsiu-Fang und Sarah E. Shannon (2005). "Three Approaches to Qualitative Content Analysis”. In: Qualitative Health Research 15.9, S. 1277-1288. DoI: 10.1177/1049732305276687 (siehe S. 100, 101).

Hug, Simon (2014). "Further Twenty Years of Pathologies? Is Rational Choice better than it used to be?" In: Swiss Political Science Review 20.3, S. 486-497. Dor: 10.1111/spsr.12123 (siehe S. 86).

Hyndman, Rob J. und George Athanasopoulos (2013). Forecasting: principles and practice. OTexts. ISBN: 978-0-9875071-0-5 (siehe S. 111).

Imhof, Kurt (2006a). "Mediengesellschaft und Medialisierung”. In: Medien und Kommunikationswissenschaft 54.2, S. 191-215 (siehe S. 29, 75, 79).

- (2006b). Politik im „neuen“Strukturwandel der Öffentlichkeit. fög / Universität Zürich (siehe S. 65).

- (2008). "Die seismographische Qualität der Öffentlichkeit”. In: Seismographische Funktion von Offentlichkeit im Wandel. Hrsg. von Heinz Bonfadelli, Kurt Imhof, Roger Blum und Otfried Jarren. VS Verlag für Sozialwissenschaften, S. 17-56. ISBN: 978-3-531-15988-1 978-3531-91126-7 (siehe S. 31). 
Immergut, Ellen M. (1998). “The Theoretical Core of the New Institutionalism”. In: Politics \& Society 26.1, S. 5-34. DoI: 10.1177/0032329298026001002 (siehe S. 87).

Inglehart, Ronald und Christian Welzel (2005). Modernization, cultural change, and democracy. Cambridge: Cambridge University Press. IsBN: 978-0-521-60971-5 (siehe S. 80).

- (2009). "How Development Leads to Democracy: What We Know About Modernization". In: Foreign Affairs 88.2, S. 33-48 (siehe S. 80).

- (2010). "Changing Mass Priorities: The Link between Modernization and Democracy". In: Perspectives on Politics 8.02, S. 551-567. Dor: 10.1017/S1537592710001258 (siehe S. 80).

Iyengar, Shanto und Adam Simon (1993). "News Coverage of the Gulf Crisis and Public Opinion A Study of Agenda-Setting, Priming, and Framing”. In: Communication Research 20.3, S. 365383. DOI: $10.1177 / 009365093020003002$ (siehe S. 70).

Janis, Irving Lester (1982). Groupthink: Psychological Studies of Policy Decisions and Fiascoes. Boston: Cengage Learning. ISBN: 978-0-395-31704-4 (siehe S. 142).

Jarren, Otfried und Patrick Donges (2011). Politische Kommunikation in der Mediengesellschaft: Eine Einführung. Wiesbaden: VS Verlag für Sozialwissenschaften. ISBN: 978-3-531-17437-2 (siehe S. 84).

Jick, Todd D. (1979). "Mixing Qualitative and Quantitative Methods: Triangulation in Action". In: Administrative Science Quarterly 24.4, S. 602-611. Dor: 10.2307/2392366 (siehe S. 96, 113). Johnson, R. Burke und Anthony J. Onwuegbuzie (2004). "Mixed Methods Research: A Research Paradigm Whose Time Has Come”. In: Educational Researcher 33.7, S. 14-26. DOI: 10.3102/0 013189X033007014 (siehe S. 95).

Jones, Bryan D. (1999). “Bounded Rationality”. In: Annual Review of Political Science 2.1, S. 297321. DoI: 10.1146/annurev.polisci.2.1.297 (siehe S. 84).

Jones, Bryan D. und Frank R. Baumgartner (2005). The politics of attention: How government prioritizes problems. Chicago: University of Chicago Press. IsBN: 978-0-226-40653-4 (siehe S. 71).

Jordan, M. I. und T. M. Mitchell (2015). "Machine learning: Trends, perspectives, and prospects". In: Science 349.6245, S. 255-260. DoI: 10.1126/science.aaa8415 (siehe S. 184).

Jost, Pablo B., Michael Sülflow und Marcus Maurer (2015). “Das können Sie morgen in der Zeitung lesen"“" In: Publizistik 60.1, S. 5-20. DoI: 10.1007/s11616-015-0225-9 (siehe S. 63).

Kahneman, Daniel (1994). "New Challenges to the Rationality Assumption". In: fournal of Institutional and Theoretical Economics (FITE) / Zeitschrift für die gesamte Staatswissenschaft 150.1, S. 18-36 (siehe S. 84). 
Kahneman, Daniel (2003). "Maps of Bounded Rationality: Psychology for Behavioral Economics”. In: The American Economic Review 93.5, S. 1449-1475 (siehe S. 84).

Kam, Christopher (2001). "Do Ideological Preferences Explain Parliamentary Behaviour? Evidence from Great Britain and Canada”. In: The fournal of Legislative Studies 7.4, S. 89-126. DOI: 10.1080/714003894 (siehe S. 56).

Kamber, Esther und Kurt Imhof (2011). Medienkonzentration und Meinungsvielfalt. Zürich: fög (siehe S. 42).

Kelly, Mary (1984). "Influences on Broadcasting Policies for Election Coverage". In: Communicating to Voters: Television in the First European Parliamentary Elections. Hrsg. von Jay G. Blumler. London ; Beverly Hills: SAGE Publications, S. 65-82. IsBN: 978-0-8039-9762-2 (siehe S. 198).

Kelly, Thomas (2003). "Epistemic Rationality as Instrumental Rationality: A Critique”. In: Philosophy and Phenomenological Research 66.3, S. 612-640 (siehe S. 34).

Kepplinger, Hans Mathias und Simone Christine Ehmig (2006). "Predicting news decisions. An empirical test of the two-component theory of news selection”. In: Communications 31.1, S. 25-43 (siehe S. 49).

Keysar, Boaz, Sayuri L. Hayakawa und Sun Gyu An (2012). "The Foreign-Language Effect Thinking in a Foreign Tongue Reduces Decision Biases”. In: Psychological Science 23.6, S. 661-668. DoI: 10.1177/0956797611432178 (siehe S. 117).

Khabsa, Madian und C. Lee Giles (2014). "The Number of Scholarly Documents on the Public Web”. In: PLoS ONE 9.5, e93949. DOI: 10.1371/journal.pone.0093949 (siehe S. 54).

Kiewiet, D. Roderick und Mathew D. McCubbins (1991). The Logic of Delegation: Congressional Parties and the Appropriations Process. Chicago: University Of Chicago Press. IsBn: 978-0226-43531-2 (siehe S. 39).

Kim, Sei-Hill, Miejeong Han, Doo-Hun Choi und Jeong-Nam Kim (2012). "Attribute agenda setting, priming and the media's influence on how to think about a controversial issue". In: International Communication Gazette 74.1, S. 43-59 (siehe S. 70).

Kim, Sei-Hill, Dietram A. Scheufele und James Shanahan (2002). "Think about it This Way: Attribute Agenda-Setting Function of the Press and the public's Evaluation of a Local Issue”. In: fournalism \& Mass Communication Quarterly 79.1, S. 7-25. DOI: 10.1177/10776990020790 0102 (siehe S. 69).

Kingdon, John W. (2010). Agendas, Alternatives, and Public Policies, Update Edition, with an Epilogue on Health Care. Boston: Pearson. ISBN: 978-0-205-00086-9 (siehe S. 71). 
Knoepfel, Peter, Yannis Papadopoulos, Pascal Sciarini, Adrian Vatter und Silja Häusermann (2014). Handbuch der Schweizer Politik Manuel de la politique suisse: 5., völlig überarbeitete und erweiterte Auf lage. Zürich: Neue Zürcher Zeitung NZZ Libro. IsBN: 978-3-03823-866-9 (siehe S. 100).

Koelble, Thomas A. (1995). “The New Institutionalism in Political Science and Sociology”. In: Comparative Politics 27.2, S. 231-243. DoI: 10.2307/422167 (siehe S. 87).

Kovic, Marko und Marc Sele (2014). "Der Umbruch der Schweizer Zeitungslandschaft in 5 Grafiken”. In: Tages-Anzeiger.ch / Datenblog (siehe S. 42, 43).

Kriesi, Hanspeter und Regula Hänggli (2012). "Frame Construction and Frame Promotion (Strategic Framing Choices)". In: American Behavioral Scientist 56.3, S. 260-278 (siehe S. 63).

Krippendorff, Klaus (2004). "Reliability in Content Analysis". In: Human Communication Research 30.3, S. 411-433. DoI: 10.1111/j.1468-2958.2004.tb00738.x (siehe S. 100).

Krotz, Friedrich (2007). Mediatisierung: Fallstudien zum Wandel von Kommunikation. SpringerVerlag. ISBN: 978-3-531-15073-4 (siehe S. 77).

Kuckartz, Udo (2015). MAXQDA, software for qualitative data analysis. Berlin (siehe S. 113).

Kuklinski, James H. und Richard C. Elling (1977). "Representational Role, Constituency Opinion, and Legislative Roll-Call Behavior". In: American fournal of Political Science 21.1, S. 135-147. DOI: $10.2307 / 2110452$ (siehe S. 56).

Künzler, Matthias (2012). "Leitmedien: Begriff, Forschungsstand, Forschungspotenzial”. In: Die Medienindustrie in der Krise. Hrsg. von Projektverbund Sinergia "Medienkrise". Universität Zürich: SwissGIS, S. 9-24 (siehe S. 104).

Künzler, Matthias, Manuel Puppis, Corinne Schweizer und Samuel Studer (2013). MonitoringReport «Medienförderung». Zürich: IPMZ (siehe S. 197).

Laffont, Jean-Jacques und David Martimort (2002). The Theory of Incentives: The Principal-Agent Model. Princeton, N.J: Princeton University Press. IsBn: 978-0-691-09184-6 (siehe S. 39).

Landerer, Nino (2013). "Rethinking the Logics: A Conceptual Framework for the Mediatization of Politics”. In: Communication Theory 23.3, S. 239-258. Dor: 10.1111/comt.12013 (siehe S. 49, 62).

- (2014). "Opposing the Government but Governing the Audience?" In: fournalism Studies 15.3, S. 304-320. DOI: 10.1080/1461670X.2014.889466 (siehe S. 60).

Langman, Lauren (2005). "From Virtual Public Spheres to Global Justice: A Critical Theory of Internetworked Social Movements*”. In: Sociological Theory 23.1, S. 42-74. DOI: 10.1111/j.07 35-2751.2005.00242.x (siehe S. 29). 
Lasswell, Harold Dwight (1956). The decision process: seven categories of functional analysis. Bureau of Governmental Research, College of business und Public Administration, University of Maryland (siehe S. 31).

Leech, Nancy L. und Anthony J. Onwuegbuzie (2007). “A typology of mixed methods research designs”. In: Quality \& Quantity 43.2, S. 265-275. Dor: 10.1007/s11135-007-9105-3 (siehe S. 96).

Leonarz, Martina (2015). “«Pressevielfalt sichern» Folgen eines Postulats”. In: Wissenschaftliche Und Praktische Medienpolitik Als Politische Daueraufgabe: Umstrittene Reaktion Auf Die Ausdunnung Des Bannwaldes Der Demokratie in Der Schweiz. Hrsg. von Martina Leonarz. Baden-Baden: Nomos Verlagsgesellschaft, S. 15-34. ISBN: 978-3-8487-1035-5 (siehe S. 42).

Leonarz, Martina, Werner A. Meier, Cinzia Dal Zotto, Benedetta Prario, Pietro Rossi und Josef Trappel (2011). Pluralismus und Vielfalt in Regionalzeitungen. Auswirkungen von Medienkonzentration und Medienkrise auf die Lokalberichterstattung in ausgewählten Regionen in der Schweiz. (Siehe S. 42).

Levitt, Steven D. (1996). "How Do Senators Vote? Disentangling the Role of Voter Preferences, Party Affiliation, and Senator Ideology". In: The American Economic Review 86.3, S. 425-441 (siehe S. 55).

Lijphart, Arend (2012). Patterns of Democracy: Government Forms and Performance in Thirty-Six Countries. New Haven: Yale University Press. IsBN: 978-0-300-17202-7 (siehe S. 31).

Lippmann, Walter (1922). Public Opinion. New York: Harcourt, Brace und Company (siehe S. 49). - (1927). The Phantom Public. Transaction Publishers. IsBn: 978-1-56000-677-0 (siehe S. 27).

Liu, Hua, Franck Prugnolle, Andrea Manica und François Balloux (2006). “A Geographically Explicit Genetic Model of Worldwide Human-Settlement History”. In: The American fournal of Human Genetics 79.2, S. 230-237. DOI: 10.1086/505436 (siehe S. 32).

Locke, John (1689). Two Treatises of Government. London: Awnsham Churchill (siehe S. 36).

Lovett, Frank (2006). "Rational Choice Theory and Explanation”. In: Rationality and Society 18.2, S. 237-272. DOI: 10.1177/1043463106060155 (siehe S. 86).

Luhmann, Niklas (1984). Soziale Systeme: Grundriss einer allgemeinen Theorie. Suhrkamp. ISBN: 978-3-518-57700-4 (siehe S. 31).

- (1996). Die Realität der Massenmedien. Opladen: VS Verlag für Sozialwissenschaften. ISBN: 978-3-531-12841-2 (siehe S. 31).

Luna, David, Torsten Ringberg und Laura A. Peracchio (2008). "One Individual, Two Identities: Frame Switching among Biculturals". In: Journal of Consumer Research 35.2, S. 279-293. DoI: 10.1086/586914 (siehe S. 117). 
Manin, Bernard (1997). The Principles of Representative Government. Cambridge ; New York: Cambridge University Press. IsBN: 978-0-521-45258-8 (siehe S. 36).

Marschall, Stefan (2001). "Das Parlament in der Mediengesellschaft - Verschränkungen zwischen parlamentarischer und massenmedialer Arena". In: Politische Vierteljahresschrift 42.3 (siehe S. 65).

- (2002). "Parlamentarische Öffentlichkeit - Eine Feldskizze". In: Der Deutsche Bundestag im Wandel. Hrsg. von Heinrich Oberreuter, Uwe Kranenpohl und Martin Sebaldt. VS Verlag für Sozialwissenschaften, S. 168-186. ISBN: 978-3-531-33684-8 978-3-322-89196-9 (siehe S. 40).

- (2003). "Strukturwandel der parlamentarischen Öffentlichkeit". In: Zeitschrift für Politik. NEUE FOLGE 50.4, S. 423-437 (siehe S. 40).

- (2009). "Medialisierung komplexer politischer Akteure - Indikatoren und Hypothesen am Beispiel von Parlamenten”. In: Politik in der Mediendemokratie. Hrsg. von Frank Marcinkowski und Barbara Pfetsch. Wiesbaden: Verlag für Sozialwissenschaften, S. 205-223. ISBN: 978-3-531-15939-3 (siehe S. 65).

Maslow, Abraham Harold (1966). The psychology of science: a reconnaissance. New York: Harper \& Row (siehe S. 78).

Matthes, Jörg und Frank Esser (2013). "Mediatization Effects on Political News, Political Actors, Political Decisions, and Political Audiences". In: Democracy in the Age of Globalization and Mediatization. Hrsg. von Hanspeter Kriesi, Daniel Bochsler, Sandra Lavenex und Marc Bühlmann. Palgrave Macmillan, S. 177-201. ISBN: 978-1-137-29987-1 (siehe S. 76, 77).

Mayring, Philipp (2010). Qualitative Inhaltsanalyse Grundlagen und Techniken. Weinheim: Beltz. ISBN: 978-3-407-25533-4 3-407-25533-0 (siehe S. 100, 101, 113).

Mazzoleni, Gianpietro (1987). "Media Logic and Party Logic in Campaign Coverage: The Italian General Election of 1983”. In: European fournal of Communication 2.1, S. 81-103. Dor: 10.1177/0267323187002001005 (siehe S. 49).

Mazzoleni, Gianpietro und Winfried Schulz (1999). ""Mediatization" of Politics: A Challenge for Democracy?" In: Political Communication 16.3, S. 247-261. DoI: 10.1080/105846099198613 (siehe S. 76).

McChesney, Robert W. (2003). "The Problem of Journalism: a political economic contribution to an explanation of the crisis in contemporary US journalism”. In: Journalism Studies 4.3, S. 299-329. DOI: 10.1080/14616700306492 (siehe S. 44).

McCombs, Maxwell (2004). Setting the Agenda: The news media and public opinion. Oxford: Blackwell Publishers. ISBN: 978-0-7456-2313-9 (siehe S. 69). 
McCombs, Maxwell (2005). "A Look at Agenda-setting: past, present and future”. In: fournalism Studies 6.4, S. 543-557. DoI: 10.1080/14616700500250438 (siehe S. 69).

McCombs, Maxwell und Salma Ghanem (2001). "The Convergence of Agenda Setting and Framing”. In: Framing Public Life: Perspectives on Media and Our Understanding of the Social World. Hrsg. von Stephen D. Reese, Oscar H. Gandy Jr. und August E. Grant. London: Routledge, S. 67-82. IsBN: 978-0-8058-3653-0 (siehe S. 70).

McCombs, Maxwell, Juan Pablo Lopez-Escobar Esteban Llamas und Federico Rey (1997). "Candidate Images in Spanish Elections: Second-Level Agenda-Setting Effects”. In: fournalism \& Mass Communication Quarterly 74.4, S. 703-717 (siehe S. 69).

McCombs, Maxwell und Donald L. Shaw (1972). "The Agenda-Setting Function of Mass Media". In: Public Opinion Quarterly 36.2, S. 176-187. DoI: 10.1086/267990 (siehe S. 69, 99).

McLellan, Eleanor, Kathleen M. MacQueen und Judith L. Neidig (2003). "Beyond the Qualitative Interview: Data Preparation and Transcription”. In: Field Methods 15.1, S. 63-84. DoI: 10.117 7/1525822X02239573 (siehe S. 109).

Meier, Werner A. (2012). "Die Medienkrise als publizistikwissenschaftliche Herausforderung”. In: Gehen in den Leuchttürmen die Lichter aus? Was aus den Schweizer Leitmedien wird. Hrsg. von Werner A. Meier, Heinz Bonfadelli und Josef Trappel. Münster: LIT Verlag, S. 11-44. ISBN: 978-3-643-80136-4 (siehe S. 41).

- (2015). "Regionale Medien in Bezirken und Kantonen”. In: Wissenschaftliche Und Praktische Medienpolitik Als Politische Daueraufgabe: Umstrittene Reaktion Auf Die Ausdunnung Des Bannwaldes Der Demokratie in Der Schweiz. Hrsg. von Martina Leonarz. Baden-Baden: Nomos Verlagsgesellschaft, S. 35-70. IsBN: 978-3-8487-1035-5 (siehe S. 43).

Meier, Werner A., Martina Leonarz, Stefan Bosshart, Mirjam Inauen und Florin Büchel (2014). Politikberichterstattung in Gemeinden und Bezirken. Eine Übersicht zu Regionalmedien. Zürich: IPMZ (siehe S. 43).

Meller, Norman (1965). “"Legislative Behavior Research" Revisited: A Review of Five Years' Publications”. In: The Western Political Quarterly 18.4, S. 776-793. Dor: 10.2307/445884 (siehe S. 55).

Meraz, Sharon (2011). "Using Time Series Analysis to Measure Intermedia Agenda-Setting Influence in Traditional Media and Political Blog Networks”. In: fournalism \& Mass Communication Quarterly 88.1, S. 176-194. DOI: 10.1177/107769901108800110 (siehe S. 30).

Merriam, Sharan B. (1995). "What Can You Tell from an N of 1?: Issues of Validity and Reliability in Qualitative Research”. In: PAACE fournal of Lifelong Learning 4, S. 51-60 (siehe S. 116). 
Meyer, David E. und Roger W. Schvaneveldt (1971). "Facilitation in recognizing pairs of words: Evidence of a dependence between retrieval operations". In: fournal of Experimental Psychology 90.2, S. 227-234. DOI: 10.1037/h0031564 (siehe S. 70).

Meyer, David E., Roger W. Schvaneveldt und Margaret G. Ruddy (1974). "Functions of graphemic and phonemic codes in visual word-recognition”. In: Memory \& Cognition 2.2, S. 309-321. DOI: 10.3758/BF03209002 (siehe S. 70).

Meyer, Thomas (2001). Mediokratie. Die Kolonisierung der Politik durch die Medien. Frankfurt am Main: Suhrkamp. IsBN: 978-3-518-12204-4 (siehe S. 77).

- (2002). Media Democracy: How the Media Colonize Politics. Cambridge, UK: Polity. IsBN: 978-0-7456-2844-8 (siehe S. 49).

Miller, Gary J. (2005). "The Political Evolution of Principal-Agent Models”. In: Annual Review of Political Science 8.1, S. 203-225. DoI: 10.1146/annurev.polisci.8.082103.104840 (siehe S. 39).

Miller, Wakken E. und Donald E. Stokes (1963). “Constituency Influence in Congress". In: American Political Science Review 57.01, S. 45-56. DoI: 10.2307/1952717 (siehe S. 55).

Milner, Henry (2002). Civic Literacy: How Informed Citizens Make Democracy Work. Hanover: Tufts. ISBN: 978-1-58465-173-4 (siehe S. 37).

Mitchell, Amy (2015). State of the News Media 2015. Washington, D.C: Pew Research Center (siehe S. 41).

Mitchell, Paul (2000). "Voters and their representatives: Electoral institutions and delegation in parliamentary democracies”. In: European fournal of Political Research 37.3, S. 335-351. DoI: 10.1111/1475-6765.00516 (siehe S. 39).

Montgomery, Douglas C., Elizabeth A. Peck und G. Geoffrey Vining (2012). Introduction to Linear Regression Analysis. Hoboken, New Jersey: John Wiley \& Sons. IsBN: 978-0-470-54281-1 (siehe S. 121).

Moran-Ellis, Jo, Victoria D. Alexander, Ann Cronin, Mary Dickinson, Jane Fielding, Judith Sleney und Hilary Thomas (2006). "Triangulation and integration: processes, claims and implications”. In: Qualitative Research 6.1, S. 45-59. Dor: 10.1177/1468794106058870 (siehe S. 113).

Mudde, Cas (2007). Populist Radical Right Parties in Europe // Populist radical right parties in Europe. Cambridge: Cambridge University Press. ISBN: 978-0-521-85081-0 (siehe S. 78).

- (2010). "The Populist Radical Right: A Pathological Normalcy". In: West European Politics 33.6, S. 1167-1186. DOI: 10.1080/01402382.2010.508901 (siehe S. 78).

Myllylahti, Merja (2014). "Newspaper Paywalls-the Hype and the Reality”. In: Digital fournalism 2.2, S. 179-194. DOI: 10.1080/21670811.2013.813214 (siehe S. 42). 
Neuberger, Christoph, Christian Nuernbergk und Melanie Rischke (2007). "Weblogs und Journalismus: Konkurrenz, Ergänzung oder Integration?” In: Media Perspektiven 2, S. 196-112 (siehe S. 30).

Newton, Kenneth (2006). "May the weak force be with you: The power of the mass media in modern politics". In: European fournal of Political Research 45.2, S. 209-234 (siehe S. 77).

Neys, Wim De (2006). "Dual Processing in Reasoning Two Systems but One Reasoner”. In: Psychological Science 17.5, S. 428-433. Dor: 10.1111/j.1467-9280.2006.01723.x (siehe S. 35).

Nickerson, Raymond S. (1998). "Confirmation bias: A ubiquitous phenomenon in many guises". In: Review of General Psychology 2.2, S. 175-220. DoI: 10.1037/1089-2680.2.2.175 (siehe S. 53).

Noije, Lonneke van, Jan Kleinnijenhuis und Dirk Oegema (2008). "Loss of parliamentary control due to mediazation and europeanization: A longitudinal and cross-sectional analysis of agenda building in the United Kingdom and the Netherlands”. In: British fournal of Political Science 38.3, S. 455-478 (siehe S. 59).

Oberreuter, Heinrich (1997). "Scheinpublizität oder Transparenz? Zur Öffentlichkeit von Parlamentsausschüssen”. In: Parlamente und ihr Umfeld. Hrsg. von Winfried Steffani und Uwe Thaysen. Zeitschrift für Parlamentsfragen. VS Verlag für Sozialwissenschaften, S. 49-64. ISBN: 978-3-322-86613-4 (siehe S. 40).

O'Cathain, Alicia, Elizabeth Murphy und Jon Nicholl (2010). "Three techniques for integrating data in mixed methods studies”. In: BMF 341, S. c4587. DoI: 10.1136/bmj.c4587 (siehe S. 113). Onwuegbuzie, Anthony J. und Nancy L. Leech (2006). "Validity and Qualitative Research: An Oxymoron?" In: Quality \& Quantity 41.2, S. 233-249. DoI: 10.1007/s11135-006-9000-3 (siehe S. 116).

Page, Benjamin I., Robert Y. Shapiro, Paul W. Gronke und Robert M. Rosenberg (1984). “Constituency, Party, and Representation in Congress”. In: The Public Opinion Quarterly 48.4, S. 741-756 (siehe S. 56).

Peters, Bernhard, Hartmut Wessler und Jürgen Habermas (2007). Der Sinn von Öffentlichkeit. Frankfurt am Main: Suhrkamp. ISBN: 978-3-518-29436-9 (siehe S. 31).

Pigliucci, Massimo (2010). Nonsense on Stilts: How to Tell Science from Bunk. Chicago: University Of Chicago Press. ISBN: 978-0-226-66786-7 (siehe S. 25).

Poggione, Sarah (2004). "Exploring Gender Differences in State Legislators' Policy Preferences”. In: Political Research Quarterly 57.2, S. 305-314. Dor: 10.1177/106591290405700211 (siehe S. 56).

Pontzen, Daniel (2006). Nur Bild, BamS und Glotze?: Medialisierung der Politik. LIT Verlag Münster. ISBN: 978-3-8258-9401-6 (siehe S. 77). 
Poole, Keith T. und Howard Rosenthal (1996). "Are legislators ideologues or the agents of constituents?” In: European Economic Review 40.3-5, S. 707-717 (siehe S. 55).

Power, Greg und Rebecca A. Shoot (2013). Global Parliamentary Report. New York: United Nations Publications. ISBN: 978-92-9142-532-6 (siehe S. 37).

Przeworski, Adam, Susan C. Stokes und Bernard Manin, Hrsg. (1999). Democracy, Accountability, and Representation. Cambridge, U.K. ; New York: Cambridge University Press. IsBN: 978-0521-64616-1 (siehe S. 38).

Puppis, Manuel, Matthias Künzler und Otfried Jarren (2012). "Einleitung: Medienwandel oder Medienkrise?" In: Medienwandel oder Medienkrise? Folgen für Medienstrukturen und ihre Erforschung. Hrsg. von Otfried Jarren, Matthias Künzler und Manuel Puppis. Baden-Baden: Nomos, S. 11-24. ISBN: 978-3-8329-6664-5 (siehe S. 41).

Puppis, Manuel, Philomen Schönhagen, Silke Fürst, Brigitte Hofstetter und Mike Meissner (2014). Arbeitsbedingungen und Berichterstattungsfreiheit in journalistischen Organisationen. Fribourg: Departement für Kommunikationswissenschaft und Medienforschung DCM (siehe S. 43).

Quackenbush, Stephen (2004). "The Rationality of Rational Choice Theory”. In: International Interactions 30.2, S. 87-107. DoI: 10.1080/03050620490462595 (siehe S. 84).

R Core Team (2015). R: A Language and Environment for Statistical Computing. Vienna, Austria (siehe S. 112).

Rasmussen, Terje (2009). “The Significance of Internet Communication in Public Deliberation”. In: favnost - The Public 16.1 (siehe S. 29).

Rauchfleisch, Adrian und Marko Kovic (2016). "The Internet and Generalized Functions of the Public Sphere: Transformative Potentials From a Comparative Perspective”. In: Social Media + Society 2.2, S. 1-15. DoI: 10.1177/2056305116646393 (siehe S. 31, 32, 40).

Reese, Stephen D. (2007). "The Framing Project: A Bridging Model for Media Research Revisited". In: fournal of Communication 57.1, S. 148-154. Dor: 10.1111/j.1460-2466.2006.00334.x (siehe S. 70).

Reichenbach, Hans (1938). Experience and prediction an analysis of the foundations and the structure of knowledge. Chicago, Ill: The University of Chicago Press (siehe S. 25).

Reinemann, Carsten (2010). "Medialisierung ohne Ende? Zum Stand der Debatte um Medieneinflüsse auf die Politik". In: Zeitschrift für Politik. Neue Folge 57.3, S. 278-293 (siehe S. 77).

Rheingold, Howard (1993). The Virtual Community: Homesteading on the Electronic Frontier. MIT Press. ISBN: 978-0-262-26110-4 (siehe S. 29). 
Riesmeyer, Claudia (2011). "Das Leitfadeninterview. Königsweg der qualitativen Journalismusforschung?” In: Methoden der fournalismusforschung. Hrsg. von Olaf Jandura, Thorsten Quandt und Jens Vogelgesang. VS Verlag für Sozialwissenschaften, S. 223-236. ISBN: 978-3531-16975-0 978-3-531-93131-9 (siehe S. 107).

Robinson, Piers (2005). The CNN Effect: The Myth of News, Foreign Policy and Intervention. Routledge. ISBN: 978-1-134-51314-7 (siehe S. 58).

Romer, Thomas und Howard Rosenthal (1979). "The elusive median voter”. In: fournal of Public Economics 12.2, S. 143-170. DoI: 10.1016/0047-2727(79)90010-0 (siehe S. 192).

Rond, Mark De und Alan N. Miller (2005). "Publish or Perish Bane or Boon of Academic Life?" In: Journal of Management Inquiry 14.4, S. 321-329. Dor: 10.1177/1056492605276850 (siehe S. 189).

Roulston, Kathryn (2010). "Considering quality in qualitative interviewing”. In: Qualitative Research 10.2, S. 199-228. DoI: 10.1177/1468794109356739 (siehe S. 116).

Rousseau, Jean-Jacques (1762). Du contract social, ou, Principes du droit politique. Amsterdam: Marc-Michel Rey (siehe S. 30, 35).

Roy, J. (1999). "Polis and Oikos in Classical Athens”. In: Greece \& Rome (Second Series) 46.01, S. 1-18. DoI: 10.1017/S0017383500026036 (siehe S. 26).

Russell, Frank Michael, Marina A. Hendricks, Heesook Choi und Elizabeth Conner Stephens (2015). "Who Sets the News Agenda on Twitter?" In: Digital Journalism, S. 1-19. Dor: 10.1080/21670811.2014.995918 (siehe S. 30).

Russo, Federico und Matti Wiberg (2010). "Parliamentary Questioning in 17 European Parliaments: Some Steps towards Comparison”. In: Legislative Studies 16.2, S. 215-232 (siehe S. 59).

Sale, Joanna E. M., Lynne H. Lohfeld und Kevin Brazil (2002). "Revisiting the QuantitativeQualitative Debate: Implications for Mixed-Methods Research”. In: Quality and Quantity 36.1, S. 43-53. DoI: 10.1023/A:1014301607592 (siehe S. 96).

Samuelson, William und Richard Zeckhauser (1988). "Status quo bias in decision making”. In: Journal of Risk and Uncertainty 1.1, S. 7-59. Dor: 10.1007/BF00055564 (siehe S. 76).

Saxer, Ulrich (2012). Mediengesellschaft: Eine kommunikationssoziologische Perspektive. Wiesbaden: VS Verlag für Sozialwissenschaften. ISBN: 978-3-531-13371-3 (siehe S. 28, 76).

Saxer, Ulrich und Max Hofer (1992). "Bericht aus dem Bundeshaus" : eine Befragung von Bundeshausjournalisten und Parlamentariern in der Schweiz. Diskussionspunkt. Zürich: Seminar für Publizistikwissenschaft der Universität Zürich. ISBN: 978-3-908127-05-5 (siehe S. 60-62). 
Sayre, Ben, Leticia Bode, Dhavan Shah, Dave Wilcox und Chirag Shah (2010). "Agenda Setting in a Digital Age: Tracking Attention to California Proposition 8 in Social Media, Online News and Conventional News”. In: Policy \& Internet 2.2, S. 7-32. DoI: 10.2202/1944-2866.1040 (siehe S. 30).

Scheufele, Dietram A. und David Tewksbury (2007). "Framing, Agenda Setting, and Priming: The Evolution of Three Media Effects Models”. In: fournal of Communication 57.1, S. 9-20 (siehe S. 70).

Schmitter, Philippe C. (1974). "Still the Century of Corporatism?" In: The Review of Politics 36.1, S. 85-131 (siehe S. 186).

Schulz, Winfried (2004). "Reconstructing Mediatization as an Analytical Concept". In: European Journal of Communication 19.1, S. 87-101 (siehe S. 75).

Schvaneveldt, Roger W., David E. Meyer und Curtis A. Becker (1976). "Lexical ambiguity, semantic context, and visual word recognition”. In: fournal of Experimental Psychology: Human Perception and Performance 2.2, S. 243-256. Dor: 10.1037/0096-1523.2.2.243 (siehe S. 70).

Schwarz, Gideon (1978). "Estimating the Dimension of a Model”. In: The Annals of Statistics 6.2, S. 461-464. DoI: 10.1214/aos/1176344136 (siehe S. 121).

Schwarz, Norbert, Herbert Bless, Fritz Strack, Gisela Klumpp, Helga Rittenauer-Schatka und Annette Simons (1991). "Ease of retrieval as information: Another look at the availability heuristic". In: Journal of Personality and Social Psychology 61.2, S. 195-202. DOI: 10.1037/002 2-3514.61.2.195 (siehe S. 71).

Searle, John R. (1970). Speech acts: An essay in the philosophy of language. Cambridge: Cambridge U.P. ISBN: 978-0-521-09626-3 (siehe S. 28).

- (2006). "Social Ontology: Some basic principles". In: Anthropological Theory 6.1, S. 12-29 (siehe S. 28).

- (2010). Making the social world. Oxford: Oxford University Press. IsBN: 978-0-19-957691-3 (siehe S. 33).

Seber, George A. F. und Alan J. Lee (2012). Linear Regression Analysis. Hoboken, New Jersey: John Wiley \& Sons. ISBN: 978-1-118-27442-2 (siehe S. 121).

Seeger, Matthew W., Timothy L. Sellnow und Robert R. Ulmer (2012). "Communication, Organization, and Crisis". In: Communication Yearbook 21. Hrsg. von Michael Roloff. New York: Routledge, S. 231-276. ISBN: 978-0-415-87315-4 (siehe S. 41). 
Sell, Aaron, Edward H Hagen, Leda Cosmides und John Tooby (2006). "Evolutionary Psychology: Applications and Criticisms". In: Encyclopedia of Cognitive Science. John Wiley \& Sons, Ltd. ISBN: 978-0-470-01886-6 (siehe S. 33).

Sellers, Patrick (2009). Cycles of Spin: Strategic Communication in the U.S. Congress. Cambridge ; New York: Cambridge University Press. IsBN: 978-0-521-13580-1 (siehe S. 58).

Sen, Amartya K. (1977). "Rational Fools: A Critique of the Behavioral Foundations of Economic Theory". In: Philosophy \& Public Affairs 6.4, S. 317-344 (siehe S. 85).

Shavell, Steven (1979). "Risk Sharing and Incentives in the Principal and Agent Relationship". In: The Bell fournal of Economics 10.1, S. 55-73. Dor: 10.2307/3003319 (siehe S. 39).

Sheafer, Tamir und Gabriel Weimann (2005). "Agenda Building, Agenda Setting, Priming, Individual Voting Intentions, and the Aggregate Results: An Analysis of Four Israeli Elections”. In: Journal of Communication 55.2, S. 347-365 (siehe S. 70).

Shehata, Adam und Jesper Strömbäck (2013). "Not (Yet) a New Era of Minimal Effects A Study of Agenda Setting at the Aggregate and Individual Levels". In: The International fournal of Press/Politics 18.2, S. 234-255. DoI: 10.1177/1940161212473831 (siehe S. 99).

Shepsle, Kenneth A. (1972). "The Strategy of Ambiguity: Uncertainty and Electoral Competition". In: American Political Science Review 66.02, S. 555-568. Dor: 10.2307/1957799 (siehe S. 39).

- (1989). "Studying Institutions Some Lessons from the Rational Choice Approach". In: fournal of Theoretical Politics 1.2, S. 131-147. DoI: 10.1177/0951692889001002002 (siehe S. 86).

- (2010). Analyzing Politics: Rationality, Behavior, and Instititutions. W W Norton \& Company Incorporated. ISBN: 978-0-393-93507-3 (siehe S. 57, 83).

Siles, Ignacio und Pablo J. Boczkowski (2012). "Making sense of the newspaper crisis: A critical assessment of existing research and an agenda for future work". In: New Media \& Society 14.8, S. 1375-1394. Dor: 10.1177/1461444812455148 (siehe S. 41).

Simon, Herbert A. (1955). "A Behavioral Model of Rational Choice”. In: The Quarterly fournal of Economics 69.1, S. 99-118. DOI: 10.2307/1884852 (siehe S. 34, 84).

Smedley, Audrey und Brian D. Smedley (2005). "Race as biology is fiction, racism as a social problem is real: Anthropological and historical perspectives on the social construction of race”. In: American Psychologist 60.1, S. 16-26. Dor: 10.1037/0003-066X.60.1.16 (siehe S. 33).

Smith, Vernon L. (1991). "Rational Choice: The Contrast between Economics and Psychology". In: Journal of Political Economy 99.4, S. 877-897 (siehe S. 84).

Solove, Daniel J. (2002). “Conceptualizing Privacy”. In: California Law Review 90.4, S. 1087-1155. DOI: $10.2307 / 3481326$ (siehe S. 26). 
Soroka, Stuart, Erin Penner und Kelly Blidook (2009). "Constituency Influence in Parliament”. In: Canadian Journal of Political Science/Revue canadienne de science politique 42.03, S. 563-591. DOI: 10.1017/S0008423909990059 (siehe S. 56).

Spradley, JP (1980). Participant observation. New York: Holt, Rinehart und Winston (siehe S. 169). Spremann, Klaus (1987). “Agent and Principal”. In: Agency Theory, Information, and Incentives. Hrsg. von Günter Bamberg und Klaus Spremann. Berlin Heidelberg: Springer, S. 3-37. ISBN: 978-3-540-51675-0 (siehe S. 39).

Staab, Joachim Friedrich (1990). Nachrichtenwert-Theorie: formale Struktur und empirischer Gehalt. Alber. ISBN: 978-3-495-47686-4 (siehe S. 49).

Starr, Paul (2012). “An Unexpected Crisis The News Media in Postindustrial Democracies”. In: The International fournal of Press/Politics 17.2, S. 234-242. DoI: 10.1177/1940161211434422 (siehe S. 41).

Stringer, C. B. und P. Andrews (1988). "Genetic and fossil evidence for the origin of modern humans”. In: Science 239.4845, S. 1263-1268. DoI: 10.1126/science.3125610 (siehe S. 32).

Stringer, Chris (2003). "Human evolution: Out of Ethiopia”. In: Nature 423.6941, S. 692-695. DoI: 10.1038/423692a (siehe S. 32).

Stringer, Ernest T. (2013). Action Research. SAGE Publications. IsBN: 978-1-4833-2073-1 (siehe S. 189).

Strøm, Kaare (2000). "Delegation and accountability in parliamentary democracies”. In: European Journal of Political Research 37.3, S. 261-289. DoI: 10.1111/1475-6765.00513 (siehe S. 39).

Strömbäck, Jesper (2008). "Four Phases of Mediatization: An Analysis of the Mediatization of Politics”. In: The International fournal of Press/Politics 13.3, S. 228-246. DoI: 10.1177/1940161 208319097 (siehe S. 49, 79, 82).

Strömbäck, Jesper und Frank Esser (2009). "Shaping Politics: Mediatization and Media Interventionism". In: Mediatization: Concept, Changes, Consequences. Hrsg. von Knut Lundby. New York: Peter Lang Publishing Inc., S. 205-223. ISBN: 978-1-4331-0562-3 (siehe S. 49).

Suppe, Frederick (2000). "Understanding Scientific Theories: An Assessment of Developments, 1969-1998". In: Philosophy of Science 67, S102-S115 (siehe S. 72).

Swers, Michele L. (2002). The Difference Women Make: The Policy Impact of Women in Congress. Chicago ; London: University Of Chicago Press. IsBn: 978-0-226-78649-0 (siehe S. 56).

Takeshita, Toshio (2005). "Current Critical Problems in Agenda-Setting Research". In: Public Opinion Research 18.3, S. 275-296 (siehe S. 72).

Taleb, Nassim Nicholas (2010). The Black Swan: The Impact of the Highly Improbable. New York: Random House Trade Paperbacks. ISBN: 978-0-8129-7381-5 (siehe S. 25). 
Tan, Yue und David H. Weaver (2007). "Agenda-Setting Effects among the Media, the Public, and Congress, 1946-2004". In: Journalism \& Mass Communication Quarterly 84.4, S. 729-744. DOI: 10.1177/107769900708400405 (siehe S. 58).

Tarrow, Sidney (2001). "TRANSNATIONAL POLITICS: Contention and Institutions in International Politics”. In: Annual Review of Political Science 4.1, S. 1-20. Dor: 10.1146/annurev.polisci.4 .1 .1 (siehe S. 75).

Tausanovitch, Chris und Christopher Warshaw (2013). "Measuring Constituent Policy Preferences in Congress, State Legislatures, and Cities”. In: The fournal of Politics 75.02, S. 330342. Dor: 10.1017/S0022381613000042 (siehe S. 56).

Taylor, Andrew J. (1998). “Domestic Agenda Setting, 1947-1994”. In: Legislative Studies Quarterly 23.3, S. 373-397. Dor: 10.2307/440359 (siehe S. 65).

Templeton, Alan R. (2013). “Biological races in humans”. In: Studies in History and Philosophy of Biological and Biomedical Sciences 44.3, S. 262-271. DoI: 10.1016/j.shpsc.2013.04.010 (siehe S. 33).

Thompson, John B. (2011). "Shifting Boundaries of Public and Private Life”. In: Theory, Culture \& Society 28.4, S. 49-70. DOI: 10.1177/0263276411408446 (siehe S. 26).

Traber, Denise, Simon Hug und Pascal Sciarini (2014). "Party Unity in the Swiss Parliament: The Electoral Connection”. In: The fournal of Legislative Studies 20.2, S. 193-215. DoI: 10.1080/13572334.2013.837259 (siehe S. 56).

Tresch, Anke (2009). "Politicians in the Media: Determinants of Legislators' Presence and Prominence in Swiss Newspapers". In: The International fournal of Press/Politics 14.1, S. 6790. Dor: 10.1177/1940161208323266 (siehe S. 63).

Tulving, E. und D. L. Schacter (1990). "Priming and human memory systems". In: Science 247.4940, S. 301-306. DOI: 10.1126/science.2296719 (siehe S. 70).

Tversky, Amos und Daniel Kahneman (1973). "Availability: A heuristic for judging frequency and probability”. In: Cognitive Psychology 5.2, S. 207-232. DoI: 10.1016/0010-0285(73)90033-9 (siehe S. 71).

- (1986). "Rational Choice and the Framing of Decisions". In: The Journal of Business 59.4, S251-S278 (siehe S. 34, 70, 84).

Uman, Lindsay S. (2011). "Systematic Reviews and Meta-Analyses". In: Journal of the Canadian Academy of Child and Adolescent Psychiatry 20.1, S. 57-59 (siehe S. 53).

Uslaner, Eric M. und Ronald E. Weber (1979). "U. S. State Legislators' Opinions and Perceptions of Constituency Attitudes”. In: Legislative Studies Quarterly 4.4, S. 563-585. DoI: 10.2307/439405 (siehe S. 55). 
Van Aelst, Peter, Adam Sehata und Arjen Van Dalen (2010). "Members of Parliament: Equal Competitors for Media Attention? An Analysis of Personal Contacts Between MPs and Political Journalists in Five European Countries”. In: Political Communication 27.3, S. 310325. DOI: 10.1080/10584609.2010.496711 (siehe S. 59, 60).

Van Aelst, Peter und Rens Vliegenthart (2014). "Studying the Tango". In: Journalism Studies 15.4, S. 392-410. DOI: 10.1080/1461670X.2013.831228 (siehe S. 60, 63).

Van Aelst, Peter und Stefaan Walgrave (2011). "Minimal or Massive? The Political AgendaSetting Power of the Mass Media According to Different Methods”. In: International fournal of Press/Politics 16.3, S. 295-313 (siehe S. 58, 95).

Van Santen, Rosa, Luzia Helfer und Peter Van Aelst (2013). "When politics becomes news: An analysis of parliamentary questions and press coverage in three West European countries". In: Acta Politica. DoI: 10.1057/ap.2013.33 (siehe S. 60, 63).

Vliegenthart, Rens und Noemi Mena Montes (2014). "How Political and Media System Characteristics Moderate Interactions between Newspapers and Parliaments Economic Crisis Attention in Spain and the Netherlands". In: The International fournal of Press/Politics 19.3, S. 318-339. DoI: 10.1177/1940161214531335 (siehe S. 60).

Vowe, Gerhard (2006). "Mediatisierung der Politik? Ein theoretischer Ansatz auf dem Prüfstand". In: 51.4, S. 437-455 (siehe S. 77).

Vowe, Gerhard und Marco Dohle (2009). "Weltsicht und Medienbild des Parlaments im Wandel. Eine Inhaltsanalyse von Bundestagsdebatten aus 50 Jahren”. In: Politik in der Mediendemokratie. Hrsg. von Frank Marcinkowski und Barbara Pfetsch. Wiesbaden: Verlag für Sozialwissenschaften, S. 224-250. IsBN: 978-3-531-15939-3 (siehe S. 65).

Walgrave, Stefaan, Stuart Soroka und Michiel Nuytemans (2008). “The Mass Media's Political Agenda-Setting Power: A Longitudinal Analysis of Media, Parliament, and Government in Belgium (1993 to 2000)”. In: Comparative Political Studies 41.6, S. 814-836 (siehe S. 59).

Walgrave, Stefaan und Peter Van Aelst (2006). "The Contingency of the Mass Media's Political Agenda Setting Power: Toward a Preliminary Theory”. In: Journal of Communication 56.1, S. 88-109 (siehe S. 73).

Walgrave, Stefaan und Rens Vliegenhart (2011). "Content Matters: The Dynamics of Parliamentary Questioning in Belgium and Denmark". In: Comparative Political Studies 44.8, S. 1031-1059 (siehe S. 59, 63).

Wanta, Wayne und Salma Ghanem (2006). "Effects of Agenda Setting”. In: Mass Media Effects Research: Advances Through Meta-analysis. Hrsg. von Raymond W. Preiss, Barbara Mae 
Gayle, Nancy Burrell, Mike Allen und Jennings Bryant. Mahwah, N.J: Routledge. ISBN: 978-0-8058-4999-8 (siehe S. 69).

Warren, Carl (1934). Modern news reporting. Published for the United States Armed Forces Institute by Harper \& Bros (siehe S. 49).

Waterman, David und Sung Wook Ji (2012). "Online Versus Offline in the United States: Are the Media Shrinking?” In: The Information Society 28.5, S. 285-303. Dor: 10.1080/01972243.2 012.708710 (siehe S. 41).

Weaver, David H. (2007). “Thoughts on Agenda Setting, Framing, and Priming”. In: fournal of Communication 57.1, S. 142-147. DOI: 10.1111/j.1460-2466.2006.00333.x (siehe S. 70).

Weaver, David und Swanzy Nimley Elliott (1985). "Who Sets the Agenda for the Media? A Study of Local Agenda-Building”. In: Journalism Quarterly 62.1, S. 87-94 (siehe S. 72).

Weber, Max (1919). Geistige Arbeit als Beruf, Vier Vorträge vor dem freistudentischen Bund. Munchen und Leipzig: Duncker \& Humblot (siehe S. 67).

- (2006). Wirtschaft und Gesellschaft. Paderborn: Voltmedia. IsBn: 978-3-938478-72-1 (siehe S. $25,31,34)$.

Weintraub, Jeff (1997). “The Theory and Politics of the Public / Private Distinction”. In: Public and Private in Thought and Practice: Perspectives on a Grand Dichotomy. Chicago: University of Chicago Press, S. 1-42. IsBn: 978-0-226-88624-4 (siehe S. 26).

Weiss, Robert S. (1995). Learning From Strangers: The Art and Method of Qualitative Interview Studies. Simon und Schuster. ISBN: 978-1-4391-0698-3 (siehe S. 106).

Weßler, Hartmut, Bernhard Peters und Jürgen Habermas (2007). Der Sinn von Öffentlichkeit. Frankfurt am Main: Suhrkamp Verlag. ISBN: 978-3-518-29436-9 (siehe S. 26).

Wien, Charlotte und Christian Elmelund-Præstekær (2008). "What's the Fuss About? The Interplay of Media Hypes and Politics". In: International fournal of Press/Politics 13.3, S. 247266 (siehe S. 126).

- (2009). "An Anatomy of Media Hypes: Developing a Model for the Dynamics and Structure of Intense Media Coverage of Single Issues”. In: European fournal of Communication 24.2, S. 183-201 (siehe S. 126).

Winch, Peter (1990). The Idea of a Social Science and Its Relation to Philosophy. Routledge. ISBN: 978-0-415-42358-8 (siehe S. 25).

Wirth, Werner, Jörg Matthes, Christian Schemer, Martin Wettstein, Thomas Friemel, Regula Hänggli und Gabriele Siegert (2010). "Agenda Building and Setting in a Referendum Campaign: Investigating the Flow of Arguments among Campaigners, the Media, and the 
Public". In: fournalism \& Mass Communication Quarterly 87.2, S. 328-345. DOI: 10.1177/1077 69901008700207 (siehe S. 72).

Zajonc, Robert B. (1968). “ATTITUDINAL EFFECTS OF MERE EXPOSURE.” In: fournal of Personality and Social Psychology 9.2, Pt.2, S. 1-27. Dor: 10.1037/h0025848 (siehe S. 71).

- (2001). "Mere Exposure: A Gateway to the Subliminal". In: Current Directions in Psychological Science 10.6, S. 224-228. Dor: 10.1111/1467-8721.00154 (siehe S. 71).

Zhu, Weimo (2012). "Sadly, the earth is still round ( $<0.05)$ ". In: fournal of Sport and Health Science 1.1, S. 9-11. DOI: 10.1016/j.jshs.2012.02.002 (siehe S. 121). 


\section{A Anhang}

\section{A.1 Codierleitfaden für die Inhaltsanalyse der Zeitungen}

Der vorliegende Codierleitfaden für die Inhaltsanalyse der Zeitungen ist die weitgehend unveränderte Fassung des Codierleitfadens, wie er für die Inhaltsanalyse im Codierteam verwendet wurde. Der einzige Unterschied des hier zusammengefassen Codierleitfadens zu der Fassung, wie sie in der heissen Codierung verwendet wurde, ist die Abwesenheit von Bildern in der vorliegenden Version. In der Fassung für die heisse Codierung wurde die Codierregeln durch Ausschnitte aus Zeitungen veranschaulicht. Aus Platzgründen lasse ich diese hier weg.

\section{A.1.1 Codierrelevante Inhalte: Was wird codiert?}

Bevor die einzelnen Variablen und ihre Codierkonventionen besprochen werden, muss für Zeitungen geklärt werden, was eigentlich codiert wird - es wird nämlich nicht der gesamte Zeitungsinhalt codiert.

Weitgehend wird das codiert, was intuitiv als «Artikel» beschrieben werden kann: Inhalte, welche von der Zeitung selber im redaktionellen Teil veröffentlicht werden. Folgende Zeitungsinhalte werden grundsätzlich nicht codiert:

- Inserate

- Anrisse / Inhaltsverzeichnisse

- TV- / Radioprogramme

- Veranstaltungen / Veranstaltungskalender

- Todesanzeiger

- Horoskope

- Impressum

- Roman / Rätsel / Unterhaltung

- Börsenkurse

- Wetter

- Stellenanzeiger

- Eigenwerbung (z.B. Verweis auf Inhalte der Zeitungs-Webseite)

- Korrigenda (Verweis auf Fehler in früheren Ausgaben bzw. Artikeln)

Bemerkung: «Anrisse» sind Leisten, in denen auf einzelne Artikel in der Zeitung hingewiesen wird. Bekannt ist z.B. die Anrissleiste auf der Frontseite des Tages-Anzeigers, welche sich unter dem Tages-Anzeiger-Logo und über den Frontseite-Artikeln befindet. 
Wir codieren nicht alle sonstigen Artikel bzw. Beiträge, sondern nur jene mit explizitem Bezug zu Politik und explizitem Bezug zur Schweiz auf der nationalen Ebene. Dabei haben wir ein eher breites Verständnis von Politik:

- Institutionelle Akteure aus der Schweiz sowie aus anderen Staaten (z.B Regierungen, ParlamentarierInnen, uvm.).

- Internationale Organisationen (z.B. die EU).

- Zivilgesellschaftliche Akteure (z.B. Nichtregierungs-Organisationen).

- Wirtschaftliche Akteure (sofern sie in politischem Kontext thematisiert werden).

- Ausser-institutionelle Politikereignisse (z.B. Proteste).

- Symbolpolitik (z.B. Reden von PolitikerInnen bei Volksfesten).

\section{Problemfälle: Nationale vs. regionale / lokale Politik}

Prinzipiell kann alles codiert werden, was Bezug zu Politik und der Schweiz hat, inklusive Regional- und Lokalberichterstattung. Als ein einschränkendes Kriterium werden aber nur jene Inhalte codiert, welche Bezug zu Politik auf nationaler Ebene haben.

Das hat mehrere Gründe: Der Themen-Topf wird übersichtlicher, wir können sorgfältiger die weniger umfangreichen Inhalte codieren (regionale und lokale Politik umfasst teilweise deutlich mehr Artikel und Seiten als nationale Politik), vor allem aber ist eine Reduktion auf die Berichterstattung zu nationaler Politik in Anbetracht der Fragestellungen und anstehenden Auswertungen sinnvoll.

Ein Bezug zu nationaler Ebene kann durch folgende Umstände gegeben sein:

- Entsprechende Rubrik («Schweiz», «Inland» o.ä. - alle Inhalte werden codiert).

- Akteure, Insitutionen und Prozesse mit Bezug zur Nationalebene (Bundesversammlung bzw. National- und / oder Ständerat, Bundesrat, Bundesverwaltung, Bundesgericht).

Regionale und lokale Berichterstattung kann gekennzeichnet sein durch:

- Entsprechende Rubrik («Zürich und Region» o.ä.).

- Akteure, Insitutionen und Prozesse ausschliesslich auf Regionalebene und/oder Lokalebene.

- Rein zwischenkantonale Angelegenheiten (z.B. sogenannte Konkordate; gehört zu regionaler Politik.

- Bezirkspolitik (gehört zu Lokalpolitik). 
Wichtig: Auch Artikel und Beiträge auf Regional- und Lokalebene können einen Bezug zur nationalen Ebene haben. Beispielsweise, wenn eine Kantonsregierung Forderungen an den Bundesrat stellt. Oder gerade in unserem Sample: Wahlkampfberichterstattung zu Nationalratsund Ständeratswahlen. Aus diesem Grund sind die Regional- und Lokalbünde nach wie vor nach möglicherweise codierrelevanten Artikeln zu überfliegen.

Ein weiterer Problemfall können Themen sein, welche zwar zunächst regionalen Charakter haben, aber dann in einem nationalen Kontext thematisiert werden. Ein Beispiel dafür aus unserem Sample: Die Krawalle in Zürich von 2011, welche bisweilen in einem überregionalen Zusammenhang gestellt werden, indem diese Krawalle als für die Schweiz neues Phänomen besprochen werden.

Wir erachten alle Inhalte von Echo der Zeit und Tagesschau als die nationale Ebene betreffend. Das Weglassen von Artikeln zu regionaler und lokaler Berichterstattung betrifft nur Zeitungen.

\section{A.1.2 Was ist ein Artikel in der Zeitung?}

Bei Zeitungsbeiträgen codieren wir auf der Ebene einzelner Beiträge, d.h. einzelner Artikel. Artikel sind publizistische Einheiten, die sich in der Regel durch eine thematische und formalgrafische Abgeschlossenheit auszeichnen. Zeitungsartikel können anhand folgender Merkmale voneinander abgegrenzt werden:

- Titel: Der Titel (oder Überschrift) ist ein häufiges Merkmal der Integrität eines Artikels. Ein Text mit neuem Titel bedeutet in der Regel, dass ein neuer Artikel beginnt. Der Titel alleine kann aber nicht als hinreichende Bedingung gelten, weil

- Lead: Der Lead ist die Zusammenfassung eines Artikels. Er befindet sich zwischen Titel und dem Fliesstext.

- Untertitel: Auch «Subhead» genannt. Untertitel befinden sich zwischen Titel und Lead.

- Dachzeile: Auch «Overhead» genannt. Die Dachzeile ist eine Art «Obertitel»- das Analog zum Untertitel, nur oberhalb des Titels.

- Spitzmarke: Die Spitzmarke ist ein wichtiges Element und entsprechend sehr oft bei Zeitungsartikeln vorzufinden. In der Regel steht die Spitzmarke zu Beginn des Fliesstextes. Die Spitzmarke fasst üblicherweise bis zu drei Informationen zusammen: AutorIn, Datum, Ort. Die Zeichnung der Autorschaft kann in Form eines voll ausgeschriebenen Namens erfolgen, in Form eines Kürzels, als Angabe einer Nachrichtenagentur - oder sie fehlt gänzlich. Wenn nebst der Autorschaft auch noch Datum und Ort angegeben sind, handelt es sich um einen Korrespondenten-Bericht. 
Die Elemente typischer Artikel können helfen, einzelne Artikel als zu codierende Einheiten zu bestimmen. Keines dieser Elemente ist jedoch eine notwendige Bedingung für das Vorhandensein eines Artikels, was Verwirrung stiften kann. Nachfolgend einige mögliche Problemfälle.

\section{Problemfälle: Kurzmeldungen}

Kurzmeldungen sind im Prinzip nichts weiter als kleine Artikel (oft von Nachrichtenagenturen bezogene). Entsprechend kann der Umgang mit ihnen eingängig sein, da sie sich in ihrer Form nicht von regulären Artikeln unterscheiden. Wenn Kurzmeldungen mit eigenem Titel versehen sind, folgt daraus zwingendermassen, dass sie als einzelne Artikel aufgenommen werden.

Kurzmeldungen können aber auch eine andere Form annehmen, und zwar, wenn nur ein einziger Titel für eine Sammlung von Kurzmeldungen vorhanden ist. Dieser Titel hat dabei keinen inhaltlichen Bezug zu den einzelnen Kurzmeldungen. Die einzelnen Kurzmeldungen sind in einer solchen Konstellation bisweilen grafisch voneinander getrennt (z.B. mit Linien), im Zweifelsfall muss die Getrenntheit oder der Zusammenhang der Kurzmeldungen individuell durch die Lektüre der Artikel bestimmt werden.

\section{Problemfälle: «Zusammengesetzte» Artikel}

Bei bestimmten Artikel-Kompositionen kommt es zum Konflikt der Artikelelemente, wie sie in A.1.2 vorgestellt wurden. Der Konflikt besteht darin, dass einerseits ein klarer Titel für einen vermeintlichen Artikel vorhanden ist, aber andererseits die einzelnen Abschnitte mit eigenen Spitzmarken versehen sind. Für solche Fälle gilt die Regel:

- Spitzmarke $>$ andere Elemente

Das bedeutet, dass die Spitzmarke eine hinreichende Bedingung für das Vorhandensein eines eigenständigen Artikels darstellt.

\section{Problemfälle: Informationskasten}

Bei manchen Artikeln sind Zusatzkästen vorhanden, in denen zusätzliche Informationen als Ergänzung des Hauptartikels platziert sind. Das Standardvorgehen ist, dass wir diese Zusatzkästchen als Bestandteil des Hauptteils ansehen - ausser, der Kasten ist mit einer eigener Spitzmarke versehen. 


\section{Problemfälle: Schlagzeilen}

Auf der Frontseite von Zeitungen sind insbesondere bei Boulevardtiteln (de facto in erster Linie bei Blick) nicht Artikel im eigentlichen Sinn, sondern Schlagzeilen vorhanden. Wir codieren Schlagzeilen auf der Frontseite wie gewöhnliche Artikel.

\section{Problemfälle: Parlamentschronik bei der NZZ}

Eine aussergewöhnliche und für das Projekt relevante Artikelform sind die Quasi-Parlamentschroniken, welche die NZZ früher führte. Diese Artikelart ist leicht verwirrend, weil diverse (Unter-)Titel in unterschiedlicher Grösse und Formatierung vorkommen können. Hier ist erneut, wie in A.1.2 festgehalten, die Spitzmarke das Element, das den Ausschlag gibt: Wenn eine Parlamentschronik über nur eine Spitzmarke verfügt, dann codieren wir diese Chronik als nur einen Artikel.

\section{A.1.3 Variablen für Zeitungen: Zusammenfassung}

Tabelle 29 fasst die Variablen, welche wir bei Zeitungen erheben, zusammen.

Tabelle 29: Variablen für die Inhaltsanalyse der Zeitungen.

\begin{tabular}{ll}
\hline Variable & Beschreibung \\
\hline Datum & Datum der Zeitungsausgabe. \\
Seite & Seitennummer im Original. \\
Rubrik & Originalrubrik. \\
Titel & Titel des Artikels. \\
Autorschaft & Typ der Autorschaft. \\
Artikeltyp & Art des Artikels. \\
Artikelgrösse & Relative Grösse des Artikels. \\
Bildgrösse & Relative Grösse aller im Artikel vorhandenen Bilder. \\
Thema & Spezifisches Thema des Artikels. \\
Politikfeld & Politikfeld, zu welchem der Artikel passt. \\
Nationalrat & Angabe, ob der Nationalrat thematisiert wird. \\
Ständerat & Angabe, ob der Ständerat thematisiert wird. \\
Individualakteur - Name & Name des allfälligen individuellen Akteurs. \\
Individualakteur - Partei & Parteiangehörigkeit des individuellen politischen Akteurs. \\
Individualakteur - Rolle & Rolle des individuellen politischen Akteurs. \\
Inidividualakteur - Geschlecht & Geschlecht des individuellen Akteurs. \\
Kollektivakteur & Vorkommende Kollektivakteure (Parteien). \\
\hline
\end{tabular}

Im Nachfolgenden wird jede der Variablen besprochen. 


\section{A.1.4 Datum}

Datum meint das Datum der jeweiligen Zeitungsausgabe. Das Datum wird in folgendem Format erfasst:

- [TT.MM.JJJJ]

Das bedeutet, dass beispielsweise der erste Oktober 1999 als 01.10.1999 erfasst wird.

\section{A.1.5 Seite}

Seite meint die in der Zeitung angegebene Seitennummer, auf der sich der jeweilige Artikel befindet.

\section{Problemfälle: Sonderbeilagen}

Bisweilen weisen Beilagen Sonderbeilagen in Zeitungen separate Nummeriungen auf, sprich, sie beginnen wieder bei 1 . In solchen Fällen übernehmen wir einfach die Originalnummerierung.

\section{Problemfälle: Überlaufartikel}

Bei Überlaufartikeln (Artikeln, welche auf einer Seite beginnen und auf einer anderen weitergehen) wird als Seite jene Seite angegeben, auf welcher der Artikel beginnt.

\section{A.1.6 Rubrik}

Für die Variable Rubrik wird die jeweils vorhandene Originalrubrik aus der Zeitung abgetippt. Falls keine Rubrik vorhanden ist, wird die Variable nicht vergeben.

\section{A.1.7 Titel}

Titel meint den Titel, also die Überschrift, des jeweiligen Artikels. Der Titel wird von Hand 1:1 abgetippt.

\section{Problemfälle: Kurzmeldungen ohne Titel}

Kurzmeldungen können ohne eigenen Titel sein. In solchen Fällen werden die einzelnen Kurzmeldungen nummeriert, und zwar, nach folgendem Schema:

- [Kurzmeldungs-Titel]: [Erste fünf Wörter] 
[Kurzmeldungs-Titel ]ist dabei ein Platzhalter für den Titel, welchen der Kurzmeldungs-Kasten hat. [Erste fünf Wörter]ist ein Platzhalter für die Regel, dass die ersten Fünf Wörter der Kurzmeldung erfasst werden. In Fällen, in denen die Kurzmeldungen ohne übergeordneten Titel sind, wird schlicht die Regel [Erste fünf Wörter]alleine angewendet.

\section{Problemfälle: (Sonder-)Beilagen}

Beilagen in Zeitungen können die Messung der jeweiligen Themenagenda erheblich beeinflussen, weil Beilagen oft verhältnismässig lange, monothematische Texte sind. Um bei der Auswertung den Einfluss von Beilagen berücksichtigen zu können, sollen Artikel aus Beilagen als solche gekennzeichnet werden. Die Regel dazu ist:

- Beilage: [Titel des Artikels]

Somit werden alle aus Beilagen stammenden Artikel gekennzeichnet.

\section{Problemfälle: Leserbriefe ohne Titel}

Die Art und Weise, wie Leserbriefe abgedruckt sind, kann variieren:

- Jeder Leserbrief hat eine eigene Überschrift $\rightarrow$ Der Titel wird wie bei einem normalen Artikel erfasst.

- Leserbriefe haben keine Überschrift $\rightarrow$ Wir notieren die ersten fünf Wörter.

- Mehrere Leserbriefe werden unter einer Überschrift zusammengefasst $\rightarrow$ Wir notieren die ersten fünf Wörter.

Im Grunde wäre es möglich, im dritten Fall nach der Regel [Kurzmeldungs-Titel]: [Erste fünf Wörter]zu notieren, aber der Effizienz wegen notieren wir nur die ersten fünf Wörter - anhand der Variablen Artikeltyp und Autorschaft ist definitiv bestimmbar, dass es sich um Leserbriefe handelt, und eine differenziertere Notierform ist nicht nötig.

\section{A.1.8 Autorschaft}

Die Variable Autorschaft soll erfassen, ob und wenn ja welche Angaben bezüglich der Autorschaft bei einem Artikel gemacht werden. Dabei werden nicht die effektiven Angaben abgetippt, sondern einer der Codes aus Tabelle 30 eingegeben: 
Tabelle 30: Ausprägungen und Codes der Variable Autorschaft.

\begin{tabular}{ll}
\hline Ausprägung & Code \\
\hline Keine Zeichnung & 0 \\
Redaktion & 1 \\
Gastbeitrag & 2 \\
Agentur & 3 \\
LeserInnen & 4 \\
\hline
\end{tabular}

\section{Problemfälle: Agentur und Redaktion gleichzeitig}

Bei manchen Artikeln sind gleichzeitig eine Agentur und eine Zeichnung der Redaktion vorhanden, weil es sich um eine Agenturmeldung handelt, welche noch seitens der Redaktion bearbeitet (z.B. erweiter) wurde. In solchen Fällen codieren wir 1 für Redaktion.

\section{A.1.9 Artikeltyp}

Die Variable Artikeltyp soll aufzeigen, welche Art von Artikel der betroffene Artikel ist. Die Ausprägungen sind in Tabelle 31 aufgelistet:

Tabelle 31: Ausprägungen der Variable Artikeltyp.

\begin{tabular}{ll}
\hline Ausprägung & Code \\
\hline Kurzmeldung & 1 \\
Bericht & 2 \\
Interview & 3 \\
Kommentar & 4 \\
Leitartikel & 4 \\
Reportage & 5 \\
Gastbeitrag & 6 \\
Leserbrief & 7 \\
\hline
\end{tabular}

$\mathrm{Zu}$ den einzelnen Artikeltypen:

- Kurzmeldungen und Berichte sind die häufigsten Artikelformen; ein Bericht ist der Prototyp eines regulären Zeitungsartikels (verhältnismässig sachbezogene Darstellung mehr oder weniger aktueller Ereignisse).

- Interviews sind Artikel, welche explizit nach dem Muster Frage-Antwort aufgebaut sind.

- Kommentare sind kürzere Artikel (oft in Kolumnen-Form), mit denen eine Redakteurin oder ein Redakteur eine individuelle wertende Meinung zu einem mehr oder weniger aktuellen Thema abgibt. Leitartikel dagegen sind Artikel, bei denen die Haltung der 
gesamten Redaktion zu einem immer aktuellen Sachverhalt erklärt wird (typischerweise etwa bei Abstimmungen).

- Reportagen sind meist längere Artikel, in denen in Form eines persönlichen Erlebnisberichtes erzählt wird, meistens auch mit reicher Bebilderung.

- Gastbeiträge sind Artikel, welche von Autorinnen und Autoren stammen, welche explizit (z.B. am Ende des Artikels) als Nicht-Redaktionsmitglieder ausgewiesen werden.

- Leserbriefe sind, wie der Name es sagt, «Artikel», welche Leserinnen und Leser als Reaktion auf redaktionelle Artikel einreichen. Die Leserbriefseiten sind in aller Regel explizit gekennzeichnet, z.B. als «Leserbriefe», «Forum» o.ä. Bei Leserbriefen ist in der Regel der Name der schreibenden Person sowie deren Wohnort angegeben.

\section{A.1.10 Artikelgrösse}

Artikelgrösse gibt an, wie gross der jeweilige Artikel ist, und zwar im Verhältnis zu einer Gesamtseite. Das bedeutet, dass wir keine absolute Messung vornehmen (z.B. anhand der Anzahl Zeichen oder der absoluten Grösse in $\mathrm{cm}$ und $\mathrm{mm}$ ), sondern die Grösse von Artikeln nur in Bezug zur Grösse einer Seite der jeweils betroffenen Zeitung angeben.

Die Grösse einer Seite der Zeitung ist nicht die gesamte Fläche der Seite, sondern die bedruckbare Fläche der Seite. Tabelle 32 fasst die Ausprägungen der Variable Artikelgrösse zusammen:

Tabelle 32: Ausprägungen der Variable Artikelgrösse.

\begin{tabular}{ll}
\hline Ausprägung & Code \\
\hline 5\% einer ganzen seite & 0.05 \\
$10 \%$ einer ganzen seite & 0.1 \\
20\% einer ganzen seite & 0.2 \\
$30 \%$ einer ganzen seite & 0.3 \\
$\mathrm{n} \%$ einer ganzen seite & $\mathrm{n} / 100$ \\
\hline
\end{tabular}

Die Angaben der Seitengrösse geschehen ab der Grösse 0.1 immer in Schritten von 0.1. Damit findet eine Komplexitätsreduktion statt, weil nicht mehr das gesamte Spektrum der Grössen abgebildet wird. Als Annäherung sind diese Ausprägungen aber nützlich. Weil Artikel bisweilen genau auf die Grösse von Viertelseiten ausgerichtet sind, können auch Grössen wie 0.25, 0.75 u.a. vergeben werden.

Der Entscheid, ob ein Artikel eher mit 0.2 oder 0.3 codiert werden soll, erfolgt nach individueller Einschätzung und mit so wenig Reflexionsaufwand wie möglich. Ob bei zwei 
aufeinanderfolgenden Codes (hier 0.2 und 0.3) die Wahl auf den kleineren oder den grösseren fällt, ist einerlei, weil das Gesamtbild der Artikelgrösse als Schätzvariable dennoch erhalten bleibt.

Die kleinste Auspräung ist 0.05, womit Artikel, welche bis ca. 7.5\% der Fläche einer Seite ausmachen, codiert werden sollen. Das betrifft vor allem Kurzmeldungen.

\section{Problemfälle: Überlaufartikel}

Bei Überlaufartikeln (Artikeln, welche auf einer Seite beginnen und auf einer anderen weitergehen) wird die relative Grösse des gesamten Artikels über alle Seiten codiert. Die Variable ist nach oben offen.

\section{A.1.11 Bildgrösse}

Die Variable Bildgrösse soll erfassen, wie gross die bei einem Artikel vorhandenen Bilder im Vergleich zur Gesamtgrösse einer Seite sind. Die Ausprägungen sind identisch zu den Ausprägungen von Artikelgrösse:

Tabelle 33: Ausprägungen der Variable Bildgrösse.

\begin{tabular}{ll}
\hline Ausprägung & Code \\
\hline 5\% einer ganzen seite & 0.05 \\
$10 \%$ einer ganzen seite & 0.1 \\
20\% einer ganzen seite & 0.2 \\
30\% einer ganzen seite & 0.3 \\
$\mathrm{n} \%$ einer ganzen seite & $\mathrm{n} / 100$ \\
\hline
\end{tabular}

Wenn keine Bilder vorhanden sind, wir die Variable nicht codiert. Wenn mehrere Bilder vorhanden sind, wird die relative Grösse aller zusammengerechneter Bilder codiert. Beispiel: Ein Artikel hat insgesamt zwei Bilder; zwei kleine in der Grösse von rund 0.1 und ein grosses bei knapp 0.4. Das ergibt den Code 0.6.

\section{Problemfälle: Überlaufartikel}

Bei Überlaufartikeln (Artikeln, welche auf einer Seite beginnen und auf einer anderen weitergehen) wird die relative Grösse der Bilder über alle Seiten codiert. Die Variable ist nach oben offen. 


\section{Problemfälle: Grafiken und Diagramme}

Nicht jede Form der nicht-textlichen Darstellung codieren wir als Bild. Wir differenzieren zwischen zwei Arten der grafischen Elemente, und zwar aufgrund des Informationsgehaltes, welchen sie liefern.

Als Bilder behandeln wir:

- Fotografien

- Grafiken (Zeichnungen, Gemälde)

Nicht als Bilder behandeln wir:

- Diagramme

- Landkarten

\section{A.1.12 Thema}

Die Variable Thema ist die Variable mit dem höchsten Grad der individuellen Interpretationsleitung seitens der Codierenden.

Mit der Variable Thema soll das konkrete Thema des jeweiligen Artikels in so wenigen Wörtern wie möglich erfasst werden. Zu diesem Zweck codieren wir das Thema anhand folgender Regeln:

- Es werden (wenn möglich) nur Substantive verwendet.

- Es sollen drei Fragen so gut wie möglich beantwortet sein (die ersten zwei sind zentral): Was? Wer? Wo?

Zum Beispiel: Ein Artikel thematisiert das knappe Abstimmungsresultat zu einer Gesetzesvorlage über Asylhilfe im Ständerat. Das Thema könnte wie folgt notiert werden: «Abstimmung Ständerat Asylhilfe».

Bei der Art und Weise, wie das Thema zusammenfassend notiert wird, gibt es kein richtig und kein falsch. Das Ziel ist eher, so einfach wie möglich und so einheitlich wie möglich die Themen zu notieren.

Um die Einheitlicheit zu erreichen, muss unbedingt mit dem separaten Dokument «ThemenTopf» gearbeitet werden. Alle Codierenden notieren dort einerseits die Themen, welche sie setzen (inkl. einer kurzen inhaltlichen Beschreibung des Themas), und zwar genau so, wie sie sie beim Codieren setzen. Andererseits ist der Themen-Topf permanent während des Codierens 
zu konsultieren: Falls ein Thema bereits von einer anderen Codiererin oder Codierer gesetzt wurde, wird das Thema so, wie es notiert ist, auch für die eigene Codierung übernommen.

Zum Beispiel: Ich habe den oben erwähnten Artikel mit dem Thema «Abstimmung Ständerat Asylhilfe» notiert und entsprechend im Themen-Topf notiert. Eine andere Codiererin stösst auf das gleiche Thema in einer anderen Zeitung und übernimmt «Abstimmung Ständerat Asylhilfe».

\section{Problemfälle: Sport}

Sportberichterstattung wird nur codiert, wenn ein expliziter Bezug zu Politik vorhanden ist.

Fantasiebeispiel: Ein Artikel thematisiert die steuerrechtliche Situation der Fusballorganisation FIFA.

\section{Problemfälle: Klatschberichterstattung}

Klatschberichterstattung im Sinne von Berichten über Privates werden nur codiert, wenn die Personen, um welche es sich handelt, politische Akteure sind.

Fantasiebeispiel: Eine Homestory über Nationalrätin XY.

\section{Problemfälle: Mehrere Themen}

Es kann Artikel geben, welche mehrere Themen behandeln und eine eindeutige zuordnung nicht einfach ist. Bei solchen Fällen codieren wir das Thema, welches uns am zentralsten erscheint. Annäherungspunkte für Zentralität sind:

- Titel

- Lead

- Bildinhalte und Bildunterschriften

Artikel, bei denen kein Thema als roter Faden der Narration vorhanden ist, dürften aber selten sein, weil das Ziel aus journalistischer Sicht gerade ist, kohärente Geschichten zu erzählen.

\section{Problemfälle: Mehrere Themen und mehrere Akteure}

Wenn bei Artikeln mit mehreren Themen entschieden werden muss, welche die zentralste ist, kann es sein, dass die allfällig erfassten Individual- oder Kollektivakteure nicht im Kontext des gewählten Themas behandelt werden. Diesen Umstand nehmen wir in Kauf. 


\section{A.1.13 Politikfeld}

Die Variable Politikfeld betrifft nur Artikel, welche sich um Politik drehen. Als Politik erachten wir im Projekt Agendasetting im Wesentlichen jedes menschliche Handeln, welches einen Bezug zu Herstellung und Durchsetzung allgemein verbindlicher Regeln hat. Konkret umfasst unser Politik-Spektrum:

- Institutionelle Akteure aus der Schweiz sowie aus anderen Staaten (z.B Regierungen, ParlamentarierInnen, uvm.). Internationale Organisationen (z.B. die EU).

- Zivilgesellschaftliche Akteure (z.B. Nichtregierungs-Organisationen).

- Wirtschaftliche Akteure (sofern sie in politischem Kontext thematisiert werden).

- Ausser-institutionelle Politikereignisse (z.B. Proteste).

Für die Variable Politikfeld weisen wir den jeweiligen Artikel einem der vordefinierten Politikfelder zu. Tabelle 34 fasst die Politikfelder zusammen.

Tabelle 34: Ausprägungen der Variable Politikfeld.

\begin{tabular}{ll}
\hline Ausprägung & Code \\
\hline Aussenpolitik & 1 \\
Internationale Politik & 2 \\
Innere Sicherheit & 3 \\
Äussere Sicherheit & 4 \\
Einwanderungs- und Asylpolitik & 4 \\
Wirtschaftspolitik & 5 \\
Finanzpolitik & 6 \\
Infrastrukturpolitik (Energie, Verkehr, Telekommunikation) & 7 \\
Medienpolitik & 8 \\
Umwelt- und Raumordnungspolitik & 9 \\
Landwirtschaftspolitik & 10 \\
Forschungspolitik & 11 \\
Bildungspolitik & 12 \\
Sozialpolitik & 13 \\
Gesundheitspolitik & 14 \\
Kulturpolitik & 15 \\
Polity & 16 \\
Politics & 17 \\
\hline
\end{tabular}

Eine kurze Definition der einzelnen Politikfelder:

- Aussenpolitik: Aussenpolitik betrifft alle politischen Ereignisse, bei denen die Schweiz als Akteur mit anderen Staaten oder internationalen Organisationen zu tun hat. Typische 
Beispiele sind Verhandlungen zwischen der Schweiz und der EU, die Teilnahme der Schweiz an internationalen Organisationen wie der UN, Freihandelsabkommen zwischen der Schweiz und anderen Staaten, uvm.

- Internationale Politik: Als internationale Politik codieren wir alle Artikel, welche sich mit politischen Themen anderer Staaten oder internationaler Organisationen beschäftigen, wobei die Schweiz nicht involviert ist.

- Innere Sicherheit: Innere Sicherheit meint alle Themen, welche das staatliche Gewaltmonopol innerhalb der Schweiz betreffen, also z.B. die Arbeit von Polizei, Nachrichtendienst, und dergleichen.

- Äussere Sicherheit: Äussere Sicherheit betrifft Angelegenheiten der Armee. Rüstungspolitik fällt auch in diese Kategorie (An- wie auch Verkauf von Rüstungsmaterial).

- Einwanderungs- und Asylpolitik: Diese Ausprägung umfasst alle Bereiche zu Migrationsfragen.

- Wirtschaftspolitik: Als Wirtschaftspolitik werden alle Artikel codiert, welche politische Fragen zu wirtschaftlichen Angelegenheiten, also dem Tausch von Gütern und Dienstleistungen, betreffen.

- Finanzpolitik: Finanzpolitik sind jene Themen, welche sich mit Einnahmen und Ausgaben des Staates beschäftigen. Typische Themen sind Steuerfragen und staatliche Ausgabenbudgets.

- Infrastrukturpolitik (Verkehr, Energie, Telekommunikation): Klassischwerweise betrifft Infrastrukturpolitik alles Themen, bei denen es darum geht, Punkte miteinander zu verbinden. Das heisst u.a.: Strassen bauen, Stromleitungen verlegen, (Ab-)Wassersysteme erstellen, Telefonkabel ziehen. Wir behandeln Infrastrukturpolitik aber nicht nur in diesem engen Sinn als Herstellung von Infrastruktur, sondern breiter auch als Politik des Bereitstellens der Güter, welche über die Infrastruktur befördert werden (z.B. ist die Debatte um den Atomausstieg auch Infrastrukturpolitik).

- Medienpolitik: Medienpolitik betrifft Politik zu privaten wie öffentlichen Medien, in der Schweiz z.B. typischerweise Diskussionen um das SRG-Budget.

- Umwelt- und Raumordnungspolitik: Umwelt- und Raumordnungspolitik umfasst alle Artikel, welche sich mit der politischen Frage des Umgangs mit der Natur beschäftigen. Raumordnungspolitik

- Landwirtschaftspolitik: Als Landwirtschaftspolitik codieren wir alle politischen Entscheidungen rund um Landwirtschaft. D.h., es geht um die politischen Prozesse rund um das Herstellen pflanzlicher und / oder tierischer Erzeugnisse. Als "tierische Erzeugnisse" 
sind nur andere Tiere als der Mensch gemeint. Forschungspolitik: Artikel, welche Entscheidungen rund um wissenschaftliche Forschung zum Thema haben, fallen in diese Kategorie.

- Bildungspolitik: Bildungspolitik meint alle Themen, welche sich mit der Politik von Bildungseinrichtungen auf primärer, sekundärer und tertiärer Bildungsstufe.

- Sozialpolitik: Das breite Feld der Sozialpolitik umfasst alle Regulierungs- und Umverteilungsmassnahmen, mit denen Benachteiligten geholfen werden soll. Das bedeutet im Wesentlichen alle Sozialversicherungen mit Ausnahme der Krankenversicherung, welche wir unter Gesundheitspolitik codieren.

- Gesundheitspolitik: Gesundheitspolitik umfasst alle politischen Aspekte der menschlichen Gesundheit.

- Polity: Die Ausprägung Polity wird dann vergeben, wenn ein Artikel nicht politische Themen, sondern politische Institutionen thematisiert. Ein Beispiel könnte etwa sein, dass die "Zauberformel" des Bundesrates besprochen wird.

- Politics: Politics wird vergeben, wenn der Artikel politische Prozesse und weniger konkrete Inhalte reflektiert. Das typische Beispiel ist Berichterstattung zu Wahlkämpfen, bei denen nicht die politischen Positionen der Kandidierenden im Mittelpunkt stehen, sondern dern Prozess des Wahlkampfes an und für sich.

\section{Problemfälle: Berichterstattung über die Judikative}

Wenn die Judikative thematisiert wird (meistens in Form von Artikeln über Gerichtsprozesse), weisen wir die entsprechenden Artikel dem Politikfeld zu, welches dem Thema entsprechend passend ist (z.B.

\section{Problemfälle: Überschneidung von Politikfeldern}

Es ist nicht nur möglich, sondern auch wahrscheinlich, dass es immer wieder Artikel gibt, bei denen grundsätzlich mehr als ein Politikfeld vergeben werden könnte. Zum Beispiel bei einem Artikel, welcher internationale Verhandlungen betreffend Klimawandel thematisiert. Hier wäre sowohl Aussenpolitik als auch Umweltpolitik gegeben.

Für Artikel, bei denen es zu Überschneidungen von Politikfeldern kommt, wenden wir folgende Regeln an:

- Wir versuchen, den Politikkontext und das konkrete Politikproblem zu unterscheiden. 
- Der Politikkontext ist das politische Umfeld, in welches die Thematik des Artikels eingebettet ist.

- Das Politikproblem ist die konkrete Sachfrage, um welche es geht.

- Als Politikfeld vergeben wir jene Ausprägung, welche dem Politikproblem entspricht.

Um das obige Beispiel einer internationalen Klimakonferenz (mit Beteiligung der Schweiz) aufzugreifen: Der Politikkontext wäre die internationale Konferenz, also Aussenpolitik, das Politikproblem, welches innerhalb dieses Kontextes bearbeitet werden soll, aber Klimapolitik, d.h. Umweltpolitik.

\section{Problemfälle: Armee im Inlandeinsatz}

Eine aus staatstheoretischer Sicht heikle, faktisch aber immer wieder gegebene Situation ist der Einsatz der Armee im Inland. In der Schweiz wird die Armee bisher aber nicht als Mittel der Durchsetzung staatlicher Gewalt eingesetzt (d.h. in der Funktion von Polizei), sondern vor allem in zivilem Kontext. Das typische Beispiel sind Umweltkatastrophen. Analog zu den Regeln bei der Überschneidung von Politikfeldern bildet der Armeeeinsatz den Kontext, das konkrete Politikproblem wäre z.B. eine Überschwemmung und die dadurch erforderlichen Aufräumarbeiten, also Infrastrukturpolitik.

\section{Problemfälle: Forschungspolitik und Bildungspolitik bei Hochschulen}

Ein Thema, bei welchem eine Überschneidung in der Natur der Sache liegt, ist Berichterstattung über Hochschulen. Hochschulen üben einen Forschungs- wie auch einen Bildungsauftrag aus, und die Vergabe des Politikfeldes muss auch hier anhand der in Abschnitt A.1.13 dargestellten Regeln vorgenommen werden.

Meistens sollte aus dem Artikel deutlich werden, ob es um Forschung oder um Ausbildung von Studierenden geht. Wenn beide Aspekte mit gleichem Gewicht behandelt werden (z.B. bei Artikeln, welche das Verhältnis von Forschung zu Ausbildung behandeln), ist die Vergabe des Politikfeldes eine Ermessensfrage, welche aus dem Kontext des Artikels hervorgehen soll.

\section{A.1.14 Nationalrat}

Die Variable Nationalrat betrifft nur Artikel, welche explizit Bezug zum Parlament auf Bundesebene haben. 


\section{Problemfälle: Akteure aus NR thematisiert, aber NR an sich nicht}

Bei Artikeln, in denen Nationalrätinnen und -räte thematisiert werden, aber nicht in konkretem Bezug auf ein parlamentarisches Geschäft (z.B. in ausserparlamentarischen Kontexten bei Wahlkampfevents), codieren wir den Artikel für «Nationalrat» nicht mit 1.

Die Variable Nationalrat soll festhalten, wann explizit die Arbeit des Nationalrates thematisiert wird.

\section{Problemfälle: Thema und Akteure ohne Bezug zu NR, aber ein Akteur erwähnt NR}

Wenn ein Artikel thematisch keinen Bezug zum Nationalrat hat, kann es dennoch sein, dass einer der thematisierten Akteure den Nationalrat erwähnt. Eine Beläufige Erwähnung, ohne, dass der Artikel einen wirklichen thematischen Bezug zum Nationalrat hat, genügt nicht, um Nationalrat mit 1 zu codieren.

\section{A.1.15 Ständerat}

Die Variable Ständerat betrifft nur Artikel, welche explizit Bezug zum Parlament auf Bundesebene haben.

\section{Problemfälle: Akteure aus SR thematisiert, aber SR an sich nicht}

Bei Artikeln, in denen Ständerätinnen und -räte thematisiert werden, aber nicht in konkretem Bezug auf ein parlamentarisches Geschäft (z.B. in ausserparlamentarischen Kontexten bei Wahlkampfevents), codieren wir den Artikel für "Ständerat" nicht mit 1.

Die Variable Ständerat soll analog zu Nationalrat festhalten, wann explizit die Arbeit des Ständerates thematisiert wird.

\section{A.1.16 Bemerkung: Nicht-Exklusivität}

Die Variablen Nationalrat und Ständerat können beide mit «1» codiert werden, so dies im Artikel der Fall ist.

\section{Problemfälle: Thema und Akteure ohne Bezug zu SR, aber ein Akteur erwähnt SR}

Wenn ein Artikel thematisch keinen Bezug zum Ständerat hat, kann es dennoch sein, dass einer der thematisierten Akteure den Ständerat erwähnt. Eine Beläufige Erwähnung, ohne, dass der 
Artikel einen wirklichen thematischen Bezug zum Ständerat hat, genügt nicht, um Ständerat mit 1 zu codieren.

\section{A.1.17 Individualakteur: Name (1 bis 5)}

Die Variable Name betrifft nur Artikel, welche explizit Bezug zum Parlament auf Bundesebene haben. Eine notwendige Bedingung für das Codieren von Individualakteuren ist, dass im vorherigen Schritt entweder die Variable Nationalrat oder die Variable Ständerat (oder beide) mit «1» codiert wurden.

Name meint den Namen (Nachnamen) von individuellen Akteuren aus dem Parlament, welche in einem Artikel vorkommen. Wir erfassen nicht alle vorkommenden individuellen Akteure, sondern maximal 5, und zwar gemäss folgenden Regeln: Personen in Titel, Lead, Bildern und Bildunterschriften werden als erster Schritt erhoben. Wenn noch nicht 5 Akteure erhoben sind, werden weitere individuelle Akteure erfasst, und zwar "chronologisch" gemäss ihrem Vorkommen im Artikel. Das Ziel ist nicht, um jeden Preis 5 Akteure zu erheben. D.h., wenn ein Artikel nur eine Person aus dem Parlament thematisiert, wird natürlich auch nur ein Name codiert.

\section{Problemfälle: Exekutivmitglieder}

Bei Artikeln, welche das Parlament betreffen, können auch Personen vorkommen, welche nicht Mitglieder des Parlamentes, sondern der Regierung sind, typischerweise Bundesratsmitglieder. In solchen Fällen codieren wir Regierungs- oder sonstige Exekutivmitglieder mit. Dazu gehört auch die Bundeskanzlerin bzw. der Bundeskanzler (oft in der Rolle der Sprecherin bzw. des Sprechers für den Bundesrat).

\section{Problemfälle: Gleichlautende Namen}

Es kann sein, dass mehrere Individualakteure den gleichen Namen haben (und ggf. auch in derselben Partei sind). Dass kann unter Umständen bei der Auswertung verwirrend sein. Diesen Umstand nehmen wir in Kauf.

\section{A.1.18 Individualakteur: Partei (1 bis 5)}

Die Variable Partei erfasst für die bei der Variable Name festgehaltenen individuellen Akteure die (allfällige) Parteizugehörigkeit. Tabelle 35 fasst die Ausprägungen von Partei zusammen. 
Tabelle 35: Ausprägungen der Variable Partei.

\begin{tabular}{ll}
\hline Ausprägung & Code \\
\hline Schweizerische Volkspartei & SVP \\
Sozialdemokratische Partei & SP \\
Freisinnig-demokratische Partei & FDP \\
Christlichdemokratische Volkspartei & CVP \\
Die Grünen & GPS \\
Grünliberale Partei & GLP \\
Bürgerlich-demokratische Partei & BDP \\
Evangelische Volkspartei der Schweiz & EVP \\
Lega dei Ticinesi & Lega \\
Alternative Linke & AL \\
Christlich-Soziale Partei & CSP \\
\hline
\end{tabular}

\section{Problemfälle: Keine Partei angegeben}

Es ist nicht zwingend, dass die Parteizugehörigkeit individueller Akteure genannt wird. Es stellt sich die Frage, ob wir beim Codieren die Parteizugehörigkeit nachrecherchieren. Wir handeln gemäss folgenden Regeln:

- Bei Mitgliedern des Bundesrates sowie bei Mitgliedern der Bundeskanzlei (Bundesratssprecher) wird nachrecherchiert.

- Bei Mitgliedern von National- oder Ständerat wird nicht nachrecherchiert.

\section{A.1.19 Individualakteur: Rolle (1 bis 5)}

Die Variable Rolle soll, im Anschluss an die Variablen Name und Partei, erfassen, was für Rollen die erfassten individuellen Akteure ausüben. Tabelle 36 fasst die Ausprägungen der Variable zusammen.

Tabelle 36: Ausprägungen der Variable Rolle.

\begin{tabular}{ll}
\hline Ausprägung & Code \\
\hline Nicht angegeben & 0 \\
Ratsmitglied & 1 \\
Kommissionsmitglied (inkl. Kommisionspräsidium) & 2 \\
Fraktionsmitglied (inkl. Fraktionspräsidium) & 3 \\
Ratspräsidium (inkl. Vizepräsidium) & 4 \\
Exekutive & 5 \\
\hline
\end{tabular}




\section{Problemfälle: Rolle unvollständig oder gar nicht angegeben}

Die Rolle der thematisierten parlamentarischen Akteure ist nicht immer vollständig oder überhaupt angegeben. Hier stellt sich die Frage, ob wir selber zusätzlich recherchieren sollen. In Anlehnung an die Logik des Problemfalls unter Abschnitt A.1.18 gehen wir wie folgt vor:

- Für Mitglieder von National- oder Ständerat wird nicht nachrecherchiert.

Wenn also z.B. eine Nationalrätin nur als Nationalrätin ausgewiesen wird, obwohl wir wüssten, dass sie zusätzlich Kommissionspräsidentin der Kommission ist, welche bei dem interessierenden Geschäft beteiligt ist, codieren wir trotzdem nur die Rolle als Ratsmitglied. Würden wir für alle Ratsmitglieder zusätzlich recherchieren, ob sie Rollen ausüben, welche nicht explizit ausgewiesen sind, wäre dies ein grosser Aufwand.

Eine Regel für Bundesratsmitglieder erübrigt sich, weil bei ihnen bisweilen nicht die Partei angegeben ist, aber ihre Rolle als Bundesrätin oder Bundesrat im Grunde immer.

\section{A.1.20 Individualakteur: Geschlecht (1 bis 5 )}

Die Variable Geschlecht erfasst, ob der thematisierte Individualakteur eine Frau oder ein Mann ist.

Tabelle 37: Ausprägungen der Variable Geschlecht.

\begin{tabular}{ll}
\hline Ausprägung & Code \\
\hline Frau & 1 \\
Mann & 2 \\
\hline
\end{tabular}

\section{A.1.21 Kollektivakteur: Partei (1 bis 5)}

Die Variable Kollektivakteur wird nur codiert, wenn der Artikel explizit Bezug zum Parlament hat. Diese Variable erfasst, ob nebst individuellen Akteuren auch kollektive Akteure, d.h. politische Parteien, thematisiert werden. Die Ausprägungen für die Variable Kollektivakteur sind dieselben wie jene in Tabelle 35.

\section{Problemfälle: Fraktionen}

Es kann sein, dass nicht immer Parteien thematisiert werden, sondern Fraktionen (technisch gesehen sind Fraktionen und nicht Parteien die Kollektivakteure im National- und Ständerat). 
Da die Fraktionen in der Regel identisch mit der Partei sind, codieren wir Fraktionen nicht separat.

\section{A.2 Codierleitfaden für die Inhaltsanalyse von Fernsehen}

\section{A.2.1 Was wird codiert?}

Anders als bei Zeitungen ist der codierrelevante Inhalt bei Fernsehmaterial schnell bestimmt: Wir codieren alles, was wir zur Verfügung haben. Analog zu den codierrelevanten Inhalten bei Zeitungen codieren wir nicht die kompletten Ausgaben der Tagesschau, sondern nur die Beiträge mit explizitem Bezug zu Politik und zur Schweiz. D.h., dass beispielsweise Beiträge über Sport und Kultur nicht codiert werden.

\section{A.2.2 Was ist ein Beitrag?}

Die Codiereinheiten bei der Tagesschau sind einzelne "Beiträge", welche konzeptuell analog zu einzelnen Artikeln gedacht werden können.

Die Unterscheidung nach einzelnen Beiträgen ist nicht immer einfach. Nebst der gewechselten thematischen Ausrichtung sind einzelne Beitrage anhand folgender Merkmale voneinander getrennt:

- Einblendung eines neuen Beitragstitels.

- Unterschiedlicher Off-Stimmen.

- Ansage des neuen Beitragstitels bzw. dessen Paraphrasierung.

- Wechsel der Einstellung / Schnitt.

Meistens werden neue Beiträge durch eine halbnahe Einstellung der Moderatorin oder des Moderators eingeleitet. Bei Kurzmeldungen aber sind eine Reihe von Beiträgen ohne ModerationsAnsage aneinandergereiht.

\section{A.2.3 Variablen für Fernsehen: Zusammenfassung}

Tabelle 38 fasst die Variablen, welche wir bei der Tagesschau erheben, zusammen.

\section{A.2.4 Datum}

Das Datum wird auf dieselbe Art codiert wie bei Zeitungen. 
Tabelle 38: Variablen für das Codieren der Tagesschau.

\begin{tabular}{ll}
\hline Variable & Beschreibung \\
\hline Datum & Datum der Sendungsausgabe. \\
Beitragstitel & Titel des Beitrages. \\
Beitragstyp & Art des Beitrages (Schlagzeile; Bericht; Kurzmeldung) \\
Zeit Beginn & Angabe des Beginns des Beitrages. \\
Zeit Ende & Angabe des Endes des Beitrages. \\
Thema & Spezifisches Thema des Artikels. \\
Politikfeld & Politikfeld, zu welchem der Artikel passt. \\
Nationalrat & Angabe, ob der Nationalrat thematisiert wird. \\
Ständerat & Angabe, ob der Ständerat thematisiert wird. \\
Individualakteur - Name & Name der Person. \\
Individualakteur - Partei & Die Partei, zu welcher die Person gehört. \\
Individualakteur - Rolle & Rolle der thematisierten Person. \\
Inidividualakteur - Geschlecht & Geschlecht der thematisierten Person. \\
Kollektivakteur & Vorkommende Kollektivakteure (Parteien). \\
\hline
\end{tabular}

\section{A.2.5 Beitragstitel}

Der Beitragstitel ist für das Codieren der Tagesschau eine optionale Variable: Bisweilen werden Beiträge explizit mit Titeln versehen (in Form von Einblendungen insbesondere am Anfang der Sendung bei den Schlagzeilen), aber oftmals sind keine expliziten Titel gegeben. Falls Letzteres zutrifft, wird die Variable leer gelassen.

\section{A.2.6 Beitragstyp}

Tabelle 39 fasst die unterschiedlichen Beitragstypen zusammen:

Tabelle 39: Ausprägungen der Variable Beitragstypen.

\begin{tabular}{ll}
\hline Ausprägung & Code \\
\hline Schlagzeile & 1 \\
Bericht & 2 \\
Kurzmeldung & 3 \\
\hline
\end{tabular}

Schlagzeilen sind die zusammenfassenden Angaben der wichtigsten Geschichten, welche zu Beginn der Sendung gezeigt werden. Berichte sind der häufigste Beitragstyp und stellen die übliche Darstellungsform bei der Tagesschau dar (Anmoderation $\rightarrow$ Beitrag mit Videomateri$\mathrm{al} \rightarrow$ ggf. Abschluss durch Moderation). Kurzmeldungen sind kurze (in der Regel < 30 Sekunden) Beiträge ohne Anmoderation. 


\section{A.2.7 Zeit Beginn}

Die Variable Zeit Beginn erfasst, zu welchem Zeitpunkt der jeweilige Beitrag beginnt. Die Zeitangabe ist dabei die absolute Zeitangabe der Sendung wie sie als Video-Datei vorhanden ist. Das Format der Erfassung der Beginnzeit ist folgendes:

- [hh:mm:ss]

Das bedeutet, dass ein Beitrag, welcher 3.5 Minuten nach Anfang der Sendung beginnt, mit 00:03:30 codiert wird.

Das Intro und Outro der Sendung (Vorspann und Abspann) sind nicht immer stets gleich lang, u.a., weil beim Kopieren ab Archiv-Kassette der manuell zu tätigende Aufnahmestart nicht immer zum selben Zeitpunkt stattfand. Dieser Umstand kann aber ignoriert werden: Wir notieren schlicht die absoluten Zeiten des Beitragsbeginns, weil damit in Kombination mit dem absoluten Beitragsende die Dauer der einzelnen Beiträge berechnet werden kann, und andererseits die Reihenfolge der Beiträge innerhalb einer Sendung notiert wird.

\section{Problemfälle: Sekundengenaues Notieren}

Unsere kleinste Einheit der Zeiterfassung sind Sekunden. Es kann aber durchaus anspruchsvoll sein, sekundengenau zu bestimmen, wann ein Beitrag beginnt (oder auch endet).

Wir müssen aber nicht auf die Sekunde genau bestimmen, wann Beiträge beginnen (bzw. enden). Es genügt, wenn wir so genau wie möglich die Zeiten notieren; eine allgemeine Fluktuation von + oder - einigen Sekunden ist einerlei.

\section{A.2.8 Zeit Ende}

Die Variable Zeit Ende erfasst die absolute Zeit, zu der ein Beitrag endet. Die Notationsform ist dieselbe wie bei Zeit Beginn.

Hinweis: Zeit Ende bei einem Beitrag ist automatisch "Zeit Beginn” des darauffolgenden Beitrages.

\section{A.2.9 Thema}

Das Thema wird auf dieselbe Art codiert wie bei Zeitungen. 


\section{Problemfälle: Sendungsankündigung (Eigenwerbung)}

Wenn bei der Tagesschau oder bei Echo der Zeit im Laufe oder am Ende der Sendung eine Sendungsankündigung vorhanden ist, wird diese Sendungsankündigung nicht codiert.

\section{A.2.10 Politikfeld}

Das Politikfeld wird auf dieselbe Art codiert wie bei Zeitungen.

\section{A.2.11 Nationalrat}

Nationalrat wird auf dieselbe Art codiert wie bei Zeitungen.

\section{A.2.12 Ständerat}

Ständerat wird auf dieselbe Art codiert wie bei Zeitungen.

\section{A.2.13 Individualakteur: Name (1-5)}

Der Name von Individualakteuren wird auf dieselbe Art codiert wie bei Zeitungen.

\section{A.2.14 Individualakteur: Partei (1-5)}

Der Name der Partei wird auf dieselbe Art codiert wie bei Zeitungen.

\section{A.2.15 Individualakteur: Rolle (1-5)}

Die Rolle wird auf dieselbe Art codiert wie bei Zeitungen.

\section{A.2.16 Individualakteur: Geschlecht (1-5)}

Das Geschlecht wird auf dieselbe Art codiert wie bei Zeitungen.

\section{A.2.17 Kollektivakteur: Partei (1-5)}

Die Partei als Kollektivakteur wird auf dieselbe Art codiert wie bei Zeitungen. 


\section{A.3 Codierleitfaden für die Inhaltsanalyse von Radio}

\section{A.3.1 Was wird codiert?}

Ähnlich wie beim Fernsehen besteht unser Radio-Sample aus einer einzigen Sendung, dem Echo der Zeit. Auch hier codieren wir schlicht die ganze Sendung.

\section{A.3.2 Was ist ein Beitrag?}

Wiederum wie beim Fernsehen ist das Auseinanderhalten einzelner Beiträge nur dann zuverlässig möglich, wenn die einzelnen Segmente thematisch unterschieden werden. Erschwerend kommt hinzu, dass anders als bei Fernsehen und Zeitung keine visuellen Elemente vorhanden sind, welche die Identifikation von Beiträgen erleichterten.

Einige Anhaltspunkte zur Differenzierung von Beiträgen können sein:

- Unterschiedliche Erzählstimmen.

- Jingles vor oder nach einem Beitrag.

- Anmoderationen und Abschlüsse durch die oder den HauptmoderatorIn.

Obacht ist bei Kurzmeldungen geboten: Hier werden bisweilen von derselben Person eine Reihe unterschiedlicher Nachrichten verlesen.

\section{A.3.3 Variablen für Radio: Zusammenfassung}

Die Variablen für Radio sind dieselben wie die Variablen für Fernsehen. Die Variablen werden gemäss denselben Logiken und mit denselben Ausprägungen codiert. Es kommen keine neuen Ausprägungen hinzu.

\section{A.4 Codierleitfaden für das amtliche Bulletin}

\section{A.4.1 Was wird codiert?}

Für die parlamentarische Agenda arbeiten wir mit zwei Quellen: Dem amtlichen Bulletin einerseits, der Liste eingereichter Vorstösse andererseits. Im amtlichen Bulletin codieren wir separat für Nationalrat, Ständerat und die vereinigte Bundesversammlung - gemäss der Art, wie im amtlichen Bulletin diese drei Plenarformen notiert sind. Für diese drei Arenen sind die Variablen identisch. 


\section{Problemfälle: Unterbrochene Geschäfte}

Unsere grundsätzliche Codierlogik ist, die Bulletin-Inhalte auf Tagesebene zu codieren. Parlamentarische Geschäfte werden aber nicht immer am Stück auf Tages-Basis bearbeitet, sondern sie haben Unterbrüche. Folgende Konfigurationen sind denkbar:

- Ein Geschäft beginnt am Vormittag und wird am Nachmittag weitergeführt.

- Ein Geschäft beginnt an einem Tag und wir an einem anderen weitergeführt.

Im ersten Szenario wird das Geschäft als ein ganzes Geschäft behandelt; d.h. bei Variablen wie Anzahl Abstimmungen wird zusammengerechnet.

Im zweiten Szenario wird das Geschäft nur für den jeweiligen Tag codiert - wir codieren Geschäfte nicht Tages-übergreifend.

\section{A.4.2 Was ist die Codiereinheit?}

Die Codiereinheiten beim amtlichen Bulletin sind einzelne Wormeldungen. Das bedeutet, dass wir einzelne Wortmeldungen behandeln wie einzelne Artikel in Zeitungen oder einzelne Beiträge bei Fernsehen und Radio.

\section{A.4.3 Variablen für das amtliche Bulletin: Zusammenfassung}

Tabelle 40 fasst die Variablen, welche beim amtlichen Bulletin erhoben werden, zusammen.

\section{A.4.4 Datum}

Datum meint das Datum des jeweiligen Sessionstages. Das Datum wird auf dieselbe Art codiert wie bei Zeitungen.

\section{A.4.5 Geschäftsnummer (1 bis 3)}

Die Geschäftsnummer ist die einmalige Nummer, welche jedem Geschäft verliehen wird. Die Geschäftsnummern sind im amtlichen Bulletin zu Beginn der Debatte zu einem jeden Geschäft festgehalten. Die Geschäftsnummer bleibt logischerweise dieselbe für alle Wortmeldungen aus demselben Geschäft.

Manchmal wird mehr als ein Geschäft, d.h. mehr als eine Geschäftsnummer, gleichzeitig behandelt. Dann erfassen wir alle Geschäftsnummern, maximal drei (sollte sich zeigen, dass mehr als drei Geschäfte gleichzeitig behandelt werden, weiten wir die Anzahl Geschäftsnummern entsprechend aus). 
Tabelle 40: Variablen für das Codieren des amtlichen Bulletins.

\begin{tabular}{ll}
\hline Variable & Beschreibung \\
\hline Datum & Datum des Sessionstages. \\
Geschäftsnummer 1-3 & Einmalige Nummer des Geschäftes. \\
Geschäftstyp 1-3 & Typ des Geschäftes. \\
Geschäftstitel 1-3 & Titel des Geschäftes. \\
Form & Mündlich oder schriftlich. \\
Sprache & Sprache der Wortmeldung (D, F, I, R) \\
Anzahl Zeichen & Anzahl Zeichen der einzelnen Wortmeldungen. \\
Name & Name der sprechenden Person. \\
Partei & Partei der sprechenden Person. \\
Rolle & Rolle der sprechenden Person. \\
Geschlecht & Geschlecht der Person. \\
Thema & Thema des Geschäftes. \\
Politikfeld & Politikfeld des Geschäftes. \\
Abstimmungsarten & Nur Anträge; inkl. Gesamtabstimmung; inkl. Abschlussabstimmung \\
n Abstimmungen & Anzahl Abstimmungen zum Geschäft. \\
n JA & Summe aller Ja-Stimmen aller Abstimmungen. \\
n NEIN & Summer aller Nein-Stimmen alle Abstimmungen. \\
\hline
\end{tabular}

\section{A.4.6 Geschäftstyp (1 bis 3 )}

Der Geschäftstyp gibt an, um was für eine Art der parlamentarischen Arbeit es sich bei einem Geschäft handelt.

Der Geschäftstyp ist im amtlichen Bulletin entweder unterhalb oder oberhalb des Titels angegeben. Wenn es sich um parlamentarische Vorstösse handelt (parlamentarische Initiative, Interepellation, Postulat, Motion) ist der Geschäftstyp vor dem Titel angegeben.

Wir notieren die Geschäftstypen von Hand (bzw. codieren sie) und erheben die Variable induktiv. Der Geschäftstyp bleibt logischerweise für alle Wortmeldungen eines Geschäftes derselbe. Analog zu der Anzahl Geschäftsnummern variiert auch die Anzahl Geschäftstypen.

Achtung: Es kann sein, dass zwei Geschäfte unter einer Geschäftsnummer laufen.

\section{A.4.7 Form}

Die Variable Form soll erfassen, ob die Wortmeldung mündlich oder schriftlich stattfand. Die Ausprägungen sind in Tabelle 41 festgehalten.

Die Angabe, ob eine Wortmeldung schriftlich stattfand, ist in Klammern zu Beginn der Wortmeldung gegeben. Wenn eine solche Angabe fehlt (in der Mehrheit der Fälle), fand die Wortmeldung mündlich statt. 
Tabelle 41: Ausprägungen für die Variable Form.

\begin{tabular}{ll}
\hline Ausprägung & Code \\
\hline Mündlich & 1 \\
Schriftlich & 2 \\
\hline
\end{tabular}

\section{A.4.8 Geschäftstitel (1 bis 3 )}

Der Titel des jeweiligen Geschäftes. Die Variable wird für alle Wortmeldungen eines Geschäftes logischerweise identisch vergeben. Wie die Anzahl Geschäftsnummern und die Anzahl Geschäftstypen kann die Anzahl der Geschäftstitel variieren.

\section{A.4.9 Sprache}

Die Variable Sprache soll erfassen, in welcher Sprache die Wortmeldung stattfand. Die Ausprägungen sind in Tabelle 42 festgehalten:

Tabelle 42: Ausprägungen für die Variable Sprache.

\begin{tabular}{ll}
\hline Ausprägung & Code \\
\hline Deutsch & 1 \\
Französisch & 2 \\
Italienisch & 3 \\
Romanisch & 4 \\
\hline
\end{tabular}

\section{A.4.10 Anzahl Zeichen}

Die Variable Anzahl Zeichen gibt an, wie viele Zeichen in einer Wortmeldung vorhanden sind. Wir zählen Leerzeichen mit.

Achtung: Bisweilen ist in der elektronischen Fassung des amtlichen Bulletins mitten in den Wortmeldungen eine Angabe der Seitanzahl aus der gedruckten Fassung des Bulletins angegeben. Für diese Fälle ziehen wir diese Zeichen von der gesamten Summe der Zeichen ab.

\section{A.4.11 Name}

Der Name (Nachname) der sprechenden Person. 


\section{A.4.12 Partei}

Die Partei der sprechenden Person. Die Ausprägungen der Variable Partei und die entsprechenden Codes entsprechen jenen in Tabelle 35.

\section{A.4.13 Rolle}

Die Ausprägungen der Variable Rolle und die entsprechenden Codes entsprechen jenen in Tabelle 36.

\section{Problemfälle: «Fraktionsmitglied» als Standard}

Sprechende im Parlament sind meistens als Fraktionsmitglieder zu codieren. Der Grund dafür ist schlicht, dass einzelne Ratsmitglieder im Namen ihrer Fraktion sprechen, und nur solche Ratsmitglieder für «sich selber» sprechen, welche ohne Fraktion sind (eine sehr kleine Minderheit).

\section{A.4.14 Geschlecht}

Die Ausprägungen der Variable Geschlecht und die entsprechenden Codes entsprechen jenen in Tabelle 37.

\section{A.4.15 Thema}

Die Variable Thema wird für das amtliche Bulletin auf dieselbe Art codiert wir für Zeitungen. Bisweilen können die Titel der Geschäfte 1:1 als Themen gesetzt werden. Achtung: Die Themen der Geschäfte aus dem amtlichen Bulletin werden im Themen-Topf eingetragen.

\section{A.4.16 Politikfeld}

Die Ausprägungen der Variable Politikfeld und die entsprechenden Codes entsprechen jenen in Tabelle 34.

\section{A.4.17 Abstimmungsart}

Die Variable Abstimmungsarten hält fest, ob in einem Geschäft (wenn überhaupt) nur über Anträge abgestimmt wurde, oder ob auch eine Gesamt- oder eine Schlussabstimmung vorkommt. Tabelle 43 hält die Ausprägungen fest. 
Tabelle 43: Ausprägungen für die Variable Abstimmungsart.

\begin{tabular}{ll}
\hline Ausprägung & Code \\
\hline Keine Abstimmung & 0 \\
Nur Anträge & 1 \\
Gesamtabstimmung vorhanden & 2 \\
Schlussabstimmung vorhanden & 3 \\
\hline
\end{tabular}

Gesamtabstimmungen sind die Abstimmungen über die erste Beratung des Erst- oder Zweitrates. Erst die Schlussabstimmung in beiden Räten ist der Punkt, an dem über grundsätzliche Annahme oder Ablehnung abgestimmt wird.

\section{Problemfälle: Motionen, Postulate}

Über Motionen und Postulate wird oft abgestimmt, und zwar über deren Annahme oder Ablehnung. Dabei müssen wir auch festhalten, um was für Abstimmungsarten es sich handelt. Im Sinne der Logik von Abstimmungen über Anträge, Gesamt- oder Schlussabstimmungen codieren wir Abstimmungen von Motionen und Postulaten wie folgt:

- Motion $\rightarrow$ Antrag

- Postulat $\rightarrow$ Antrag

Abstimmungen über Motionen und Postulate codieren wir also immer mit 1 für Antrag. Achtung: Viele Motionen und Postulate werden stillschweigend angenommen oder abgelehnt, d.h., ohne Abstimmung. Entsprechend codieren wir in solchen Fällen keine Abstimmungen.

\section{A.4.18 n Abstimmungen}

Die Variable Anzahl Abstimmungen gibt für alle Wortmeldungen eines Geschäftes an, wie viele Abstimmungen innerhalb dieses einen Geschäftes durchgeführt wurden.

In der Online-Version des amtlichen Bulletins sind Abstimmungen durch die Symbole «Daumen hoch - Daumen runter» (nur im Nationalrat) und «Bleistift» gekennzeichnet. Beim Daumensymbol findet auf jeden Fall eine Abstimmung statt. Beim Pfeilsymbol findet möglicherweise eine Abstimmung statt.

Das bedeutet: Wir müssen beim sowohl beim National- wie auch beim Ständerat jeweils die Bleistift-Symbole nach Abstimmungen durchkontrollieren. 


\section{A.4.19 $\mathrm{n}$ JA}

Die Variable n JA erfasst die Summe aller Ja-Stimmen aller Abstimmungen innerhalb eines Geschäftes. Wenn also bei einem Geschäft insgesamt vier Abstimmungen stattfanden, wird zusammengezählt, wie viele Ja-Stimmen insgesamt bei allen vier Abstimmungen abgegeben wurden.

Die Summe der Ja-Stimmen wird für alle Wortmeldungen eines Geschäftes notiert.

\section{Problemfälle: Was sind JA-Stimmen?}

Abstimmungen im Parlament sind nicht immer eindeutig als JA oder Nein-Abstimmungen gekennzeichnet - um ein Ja oder Nein geht es erst in den Gesamt- und den Schlussabstimmungen.

Die restlichen (und mehrheitlichen) Abstimmungen betreffen Anträge. Bei Anträgen handelt es sich um inhaltliche Änderungsvorschläge, welche immer in Opposition zu der (Mehrheit) der Kommission stehen, welche die Vorlage erarbeitet bzw. geprüft hat und sie so vorschlägt.

Konkret kann es zu folgenden Konstellationen kommen:

- Mehrheit vs. Minderheit: Die Minderheit der Kommission stellt einen Antrag in Opposition zu der Mehrheit. Stimmen für die Mehrheit codieren wir als Ja-Stimmen.

- Kommission vs. Ratsmitglied XY: Einzelne Ratsmitglieder stellen Anträge in Opposition zu der Kommission. Stimmen für die Kommission codieren wir als Ja-Stimmen.

- Kommission vs. Fraktion XY: Eine Kommission stellt einen Antrag in Opposition zu der Kommission. Stimmen für die Kommission codieren wir als Ja-Stimmen.

- Kommission vs. Bundesrat: Der Bundesrat stellt einen Antrag in Opposition zu der Kommission. Stimmen für die Kommission codieren wir als Ja-Stimmen.

- Eventualabstimmung: Wenn mehr als ein Antrag zum Gleichen Teil der Vorlage vorliegt, werden in einer sogenannten Eventualabstimmung die Anträge ausgemehrt. D.h., es kann z.B. ein Antrag des Bundesrates gegen einen

- Antrag eines Ratsmitgliedes zur Abstimmung kommen. In solchen Fällen gehen wir recht pragmatisch vor: Jener Antrag, der mehr Stimmen erhält, wird als "Ja" codiert, der Antrag mit weniger Stimmen entsprechend als "Nein". Das ist nicht ganz korrekt und widerspricht dem Ja-Nein-Schema als Kommission vs. Opposition, aber es ist die am wenigsten verzerrende Variante, die Eventualabstimmungen zu erfassen (ohne das Hinzufügen neuer Variablen). 


\section{Problemfälle: Postulate, Motionen, parlamentarische Initativen}

Wenn in National- oder Ständerat nicht über Gesetzesvorlagen, sondern über Motionen, Postulate oder parlamentarische Initiativen debattiert wird, gibt es auch Abstimmungen. Wir erfassen sie wie folgt:

- Ja-Stimmen: Stimmen, welche der Empfehlung der Kommission entsprechen.

- Nein-Stimmen: Stimmen, welche gegen die Empfehlung der Kommission sind.

Bei der Abstimmung ist jeweils über dem Ergebnis festgehalten, was die Kommission empfiehlt, Annahme oder Ablehnung des Vorstosses. Das bedeutet effektiv, dass das, was wir als Ja oder Nein codieren, von der Empfehlung der Kommission abhängt.

Wenn keine Empfehlung der Kommission gegeben ist, zählen wir ganz einfach die effektiven Ja-Stimmen als Ja-Stimmen und die effektiven Nein-Stimmen als Nein-Stimmen.

\section{A.4.20 n NEIN}

Die Variable n NEIN ist dieselbe wie n JA, nur bezogen auf die Summe der Nein-Stimmen.

\section{A.5 Codierleitfaden für eingereichte Vorstösse}

\section{A.5.1 Was wird codiert?}

Bei den eingereichten Vorstössen codieren wir all jene Vorstösse, welche für die Phase des Mediensamples eingereicht wurden. Die Codiereinheiten sind entsprechend die einzelnen Vorstösse.

\section{A.5.2 Variablen für eingereichte Vorstösse: Zusammenfassung}

Tabelle 44 fasst die Variablen, welche für die eingereichten Vorstösse erhoben werden, zusammen.

\section{A.5.3 Datum}

Datum meint das Datum, an welchem der Vorstoss eingereicht wurde. Das Datum wird auf dieselbe Art codiert wie bei Zeitungen. 
Tabelle 44: Variablen für das Codieren der eingereichten Vorstösse.

\begin{tabular}{ll}
\hline Variable & Beschreibung \\
\hline Datum & Datum des Sessionstages. \\
Geschäftsnummer & Einmalige Nummer des Geschäftes. \\
Vorstosstitel & Titel des Vorstosses. \\
Vorstossart & Art des Vorstosses. \\
Anzahl Zeichen & Anzahl Zeichen der einzelnen Vorstösse. \\
Name & Name der Person, die den Vorstoss eingereicht hat. \\
Partei & Partei der Person, die den Vorstoss eingereicht hat. \\
Kammer & Ratsmitgliedschaft der einreichenden Person. \\
Geschlecht & Geschlecht der einreichenden Person. \\
Thema & Thema des Vorstosses. \\
Politikfeld & Politikfeld des Vorstosses. \\
\hline
\end{tabular}

\section{A.5.4 Geschäftsnummer}

Jeder eingereicht Vorstoss erhält eine einmalige Geschäftsnummer, welche direkt über dem Titel des Vorstosses notiert ist.

\section{A.5.5 Vorstosstitel}

Der Titel des eingereichten Vorstosses. Titel von Vorstössen werden rot unterhalb der Geschäftsnummer festgehalten.

\section{A.5.6 Vorstossart}

Tabelle 45 fasst die Vorstossarten zusammen, welche wir bei der Codierung dieser Variable beachten.

Tabelle 45: Ausprägungen der Variable Vorstossart.

\begin{tabular}{ll}
\hline Ausprägung & Code \\
\hline Anfrage & 1 \\
Interpellation & 2 \\
Postulat & 3 \\
Motion & 4 \\
Parlamentarische Initiative & 5 \\
Standesinitiative & 6 \\
\hline
\end{tabular}




\section{A.5.7 Anzahl Zeichen}

Die Variable Anzahl Zeichen gibt die Länge der Vorstösse an, und zwar inklusive Leerzeichen. Achtung: Für die Anzahl Zeichen werden nur die Zeichen der Abschnitte «Eingereichter Text» und «Begründung» gezählt, nicht aber «Stellungnahme des Bundesrates».

\section{A.5.8 Name}

Der Name (Nachname) der Person, welche den Vorstoss eingereicht hat.

\section{A.5.9 Partei}

Die Partei der Person, welche den Vorstoss eingereicht hat. Die Ausprägungen der Variable Partei und die entsprechenden Codes entsprechen jenen in Tabelle 35.

\section{A.5.10 Kammer}

Die Kammer, deren Mitglied die Person, welche den Vorstoss eingereicht hat, ist. Tabelle 46 fasst die Ausprägungen der Variable zusammen.

Tabelle 46: Ausprägungen der Variable Kammer.

\begin{tabular}{ll}
\hline Ausprägung & Code \\
\hline Nationalrat & 1 \\
Ständerat & 2 \\
\hline
\end{tabular}

\section{A.5.11 Geschlecht}

Die Ausprägungen der Variable Geschlecht und die entsprechenden Codes entsprechen jenen in Tabelle 37.

\section{A.5.12 Thema}

Die Variable Thema wird für Vorstösse auf dieselbe Art codiert wir für Zeitungen. Achtung: Die Themen der Geschäfte aus dem amtlichen Bulletin werden im Themen-Topf eingetragen.

\section{A.5.13 Politikfeld}

Die Ausprägungen der Variable Politikfeld und die entsprechenden Codes entsprechen jenen in Tabelle 34. 


\section{A.6 Codierleitfaden für die Fragestunden}

\section{A.6.1 Was wird codiert?}

Bei den Fragestunden codieren wir alle eingereichten Fragen, und zwar auch solche, welche im Nationalrat nicht mündlich beantwortet werden. Weiter codieren wir alle Wortmeldungen zu diesen Fragen im Nationalrat.

Die Fragen sind für das Jahr 2011 und teilweise 2005 nicht direkt im Bulletin enthalten, sondern nur ein Link auf die Frage in Curia Vista. Für die Jahre 1999 und teilweise 2005 sind die Fragen direkt im Bulletin enthalten.

Wir codieren die Frage auf jeden Fall, egal, ob sie separat in Curia Vista oder direkt im Bulletin enthalten ist.

\section{A.6.2 Variablen für eingereichte Vorstösse: Zusammenfassung}

Tabelle 47 fasst die Variablen, welche für die Fragestunden erhoben werden, zusammen.

Tabelle 47: Variablen für das Codieren der Fragestunden.

\begin{tabular}{ll}
\hline Variable & Beschreibung \\
\hline Datum & Datum der Einreichung. \\
Geschäftsnummer & Einmalige Nummer des Geschäftes. \\
Fragetitel & Titel der Frage. \\
Name & Name der Person(en). \\
Partei & Partei der fragenden Person. \\
Geschlecht & Geschlecht der Person(en). \\
Rolle & Rolle der Person (Ratsmitglied oder Exekutive). \\
n Zeichen & Anzahl Zeichen \\
Thema & Thema der Frage. \\
Politikfeld & Politikfeld der Frage. \\
\hline
\end{tabular}

\section{A.6.3 Datum}

Datum meint das Datum, an welchem die Frage eingereicht wurde. Das Datum wird auf dieselbe Art codiert wie bei Zeitungen.

\section{A.6.4 Geschäftsnummer}

Jede Frage ist mit einer Geschäftsnummer versehen; die Geschäftsnummer ist identisch für die Frage an sich und für das Geschäft, unter welchem die Frage im Nationalrat thematisiert wird. 


\section{A.6.5 Fragetitel}

Titel der Frage.

\section{A.6.6 Name}

Der Name betrifft zweierlei Akteure:

- Name der Person, welche die Frage formuliert.

- Name der Person, welche zur Frage sprechen.

In der Regel kommen maximal zwei individuelle Akteure vor: Die Person, welche die Frage formuliert und eine Bundesratsmitglied. Auf die Antwort des Bundesratsmitgliedes kann die Person, welche die Frage formuliert hat, selber nochmals antworten.

\section{A.6.7 Partei}

Die Partei der fragenden Person. Die Ausprägungen der Variable Partei und die entsprechenden Codes entsprechen jenen in Tabelle 35.

\section{A.6.8 Geschlecht}

Die Ausprägungen der Variable Geschlecht und die entsprechenden Codes entsprechen jenen in Tabelle 37.

\section{A.6.9 Rolle}

Bei der Rolle unterscheiden wir nach nur zwei Kategorien, welche in Tabelle 48 zusammengefasst sind.

Tabelle 48: Ausprägungen der Variable Rolle für die Fragestunden.

\begin{tabular}{ll}
\hline Ausprägung & Code \\
\hline Nationalrat & 1 \\
Exekutive & 2 \\
\hline
\end{tabular}

\section{A.6.10 $n$ Zeichen}

Anzahl Zeichen in der Frage bzw. in den Wortmeldungen zur Frage. 


\section{A.6.11 Thema}

Die Variable Thema wird für Fragestunden auf dieselbe Art codiert wir für Zeitungen. Achtung: Die Themen der Fragestunden werden im Themen-Topf eingetragen.

\section{A.6.12 Politikfeld}

Die Ausprägungen der Variable Politikfeld und die entsprechenden Codes entsprechen jenen in Tabelle 34.

\section{A.7 Interviewleitfäden für Politikerinnen und Politiker}

Der nachfolgende Leitfaden für die Gespräche mit Mitgliedern des National- und des Ständerates beschreibt, nach welchen Dimensionen und mit was für Schwerpunkten die Gespräche geführt wurden. Der Leitfaden diente allerdings nur eben als Leitfaden und nicht als fix definiertes Skript: In jedem der Gespräche habe ich als Gesprächsleiter situativ Schwerpunkte gesetzt und die einzelnen Dimensionen und zu diesen Dimensionen gehörende Punkte mit variierendem Gewicht behandelt. Besonders wichtig war es, im Gespräch auch Raum für «Unvorhergesehenes» zu belassen, also auch Themen zu behandeln, welche in dem vorliegenden Leitfaden nicht a priori definiert sind. Die vorhandenen Fragen und Unterpunkte im Leitfaden habe ich zudem nicht wortgetreu abgelesen, sondern sie jeweils auf möglichst natürliche Art in das Gespräch eingeflochten.

\section{A.7.1 Präsenz von Medien allgemein}

Medien, und damit Journalistinnen und Journalisten, begleiten die Arbeit im Parlament während Sessionen recht eng; über 100 Medienschaffende sind im Bundeshaus akkreditiert. Können Sie, ganz allgemein, beschreiben, wie Sie die Präsenz von Journalisten/Medien während Sessionen wahrnehmen?

- Bundeshaus vs. andere Orte

- Informelle Gespräche vs. Formales (z.B. Medienkonferenzen)

- Permanente «Belagerung» vs. punktueller Austausch

- Parallelismus

- Bekanntschaften / Freundschaften 


\section{A.7.2 Medialisierung}

In Ihrer politischen Tätigkeit haben Sie zwangsläufig mit Medien, also mit JournalistInnen, zu tun. Wie haben Sie gelernt, mit Medien, mit Journalisten umzugehen?

- Learning by doing

- Medientrainings

- Persönliche Kontakte zu Journalisten

- Persönliche Erfahrung im Kommunikationsbereich

Spielt es in Ihrer parlamentarischen Arbeit eine Rolle, zu antizipieren, was wie von den Medien aufgegriffen wird? Oder Dinge so aufzubereiten, dass sie von den Medien verwertet werden können?

- Plenarphasen vs. Kommissionsarbeit

Denken Sie, dass Parlamentarierinnen und Parlamentarier allgemein in ihrer Parlamentsarbeit stark an Medien ausgerichtet oder angepasst sind?

- Unterschiede Plenarphasen vs. Kommissionsarbeit

- Unterschiede zwischen den Kammern

- Rolle der Fraktionen hinsichtlich individueller Ausrichtung bzw. Anpassung an Medien Spielen Social Media für Sie eine Rolle?

- Wie?

- Ergänzung zu klassischen Medien vs. Ersatz für klassische Medien.

Spielen Social Media allgemein eine Rolle im Parlamentsbetrieb?

\section{A.7.3 Agenda-Setting allgemein}

Medien berichten nach wie vor rege über das Sessionsgeschehen. Wie wählen Medien aus, was sie aus dem Parlament aufgreifen?

- Reguläre Traktanden NR und SR

- Fragestunden

- Eingereichte Vorstösse

Wie beeinflussen Medien, wenn überhaupt, das Parlament? 
- Einfluss auf Abstimmungsergebnisse

- Einfluss auf Fragestunden

- Einfluss auf eingereichte Vorstösse

Was würden Sie schätzen, wer gibt in diesem Wechselspiel von Parlament und Medien eher den Takt an?

\section{A.7.4 Strategisches Handeln}

Nutzen Ihre Kolleginnen und Kolegen im Parlament Ihrer Meinung nach Medien gezielt, um strategisch bestimmte Ziele zu verfolgen?

- Was für Ziele sind das?

Nutzen Sie selber Medien strategisch, um Ziele zu verfolgen?

- Welche Ziele verfolgen Sie?

- Wiederwahl / Wahlkampf

- Abstimmungskampf

- Ausserparlamentarische Parteipolitik

- Persönliche Bekanntheit / Constituents

- Politikfelder / Issue Ownership

- Wichtige Themen / persönliche Überzeugung

Es ist denkbar, dass ParlamentarierInnen und Journalisten zwar strategisch miteinander umgehen, aber nur indirekt (es wird in Erwartung der Handlungen der Gegenseite vorausschauend gehandelt). Es könnte aber auch sein, dass ParlamentarierInnen und Journalisten direkt miteinander in einer Art strategischer Partnerschaft stehen. Wie schätzen Sie diese Situation ein? Gehen Sie selber strategische Partnerschaften ein?

\section{A.7.5 Normative Bewertungen}

Die Frage, wie das Verhältnis von Parlament und Medien ausfällt, ist nicht zuletzt darum relevant, weil vielleicht nicht jede Form der Wechselwirkungen demokratisch gesehen gut ist. Wie schätzen Sie diesbezüglich die Situation ein? Gibt es demokratisch gesehen problematische Dinge?

- Sichtbarkeit der parlamentarischen Arbeit 
- Strategische Partnerschaften: Partikulare Interessen vs. Interessen der Wählerschaft

- Journalistisches Selbstverständnis

- Selbstverständnis der ParlamentarierInnen

- Wer ist «schuld»: Medien oder Parlament?

\section{A.7.6 Entwicklung}

Sie haben ausführlich beschrieben, wie Sie die gegenwärtige Situation einschätzen. Was würden Sie zur Entwicklung der Situation sagen: Gab es in den letzten ca. 10 bis 20 Jahren Ihrer Meinung nach Veränderungen? (Je nach Person: Obwohl Sie selber noch nicht diese ganze Zeit im Parlament miterlebt haben.)

- Umgang mit Medien allgemein

- Medialisiertheit der ParlamentarierInnen

- Gegenseitige Beeinflussung von Medien und Parlament

- Nach unterschiedlichen Arenen

- Strategischer Umgang miteinander

- Wandel der zu erreichenden Ziele

\section{A.7.7 Abschluss}

Gibt es vielleicht noch Punkte, die wir nicht angesprochen haben, die aber wichtig sind?

\section{A.8 Interviewleitfäden für Journalistinnen und Journalisten}

Die Natur des Leitfadens für die Gespräche mit Journalistinnen und Journalisten ist dieselbe wie bei dem Leitfaden für die Gespräche mit Mitgliedern des National- und Ständerates (vgl. Unterabschnitt A.7): Der Leitfaden gibt die Stossrichtung und die inhaltliche Struktur der geführten Gespräche an, nicht aber den konkreten jeweiligen Gesprächsablauf, da dieser bewusst situativ flexibel gehandhabt wurde.

\section{A.8.1 Journalistische Arbeit im Parlament allgemein}

Medien, d.h. Journalistinnen und Journalisten, begleiten die Arbeit im Parlament während Sessionen recht eng; knapp 200 Medienschaffende sind im Bundeshaus akkreditiert. Können Sie, ganz allgemein, beschreiben, wie Ihre Arbeit während der Session aussieht?

- Physische Präsenz 
- Informelle Gespräche vs. Formales (z.B. Medienkonferenzen)

- Permanente «Belagerung» vs. punktueller Austausch

- Professionalität

- Parallelismus

- Bekanntschaften / Freundschaften

\section{A.8.2 Medialisierung}

Der Umgang mit Medien gehört zum Parlamentsgeschehen. Was würden Sie einschätzen, wie lernen ParlamentarierInnen, mit Ihnen bzw. mit Medien allgemein umzugehen?

- Learning by doing

- Medientrainings

- Persönliche Kontakte zur Journalisten

- Persönliche Erfahrung im Kommunikationsbereich

Denken Sie, dass Parlamentarierinnen und Parlamentarier in ihrer Parlamentsarbeit stark an Medien ausgerichtet oder angepasst sind?

- Plenarphasen vs. Kommissionsarbeit

- Unterschiede zwischen den Kammern

Spielen Social Media für Sie eine Rolle?

- Ergänzung zu klassischen Medien vs. Ersatz für klassische Medien.

Spielen Social Media allgemein eine Rolle im Parlametsgeschehen?

\section{A.8.3 Agenda-Setting allgemein}

Medien berichten nach wie vor rege über das Sessionsgeschehen. Wie wählen Medien, wie wählen Sie aus, was Sie aus dem Parlament aufgreifen?

- Reguläre Traktanden NR und SR.

- Fragestunden

- Eingereichte Vorstösse

Wie beeinflussen Medien, wenn überhaupt, das Parlament?

- Einfluss auf Abstimmungsergebnisse

- Einfluss auf Fragestunden

- Einfluss auf eingereichte Vorstösse 


\section{A.8.4 Strategisches Handeln}

Nutzen Parlamentarierinnen und Parlamentarier Ihrer Meinung nach Medien gezielt, um strategisch bestimmte Ziele zu verfolgen?

- Was für Ziele sind das?

Was für strategische Ziele verfolgen Sie als Journalist(in)?

Was für strategische Ziele verfolgen Medien allgemein?

Es ist denkbar, dass ParlamentarierInnen und Journalisten zwar strategisch miteinander umgehen, aber nur indirekt (es wird in Erwartung der Handlungen der Gegenseite vorausschauend gehandelt). Es könnte aber auch sein, dass ParlamentarierInnen und Journalisten direkt miteinander in einer Art strategischer Partnerschaft stehen. Wie schätzen Sie diese Situation ein?

Gehen Sie selber strategische Partnerschaften ein?

\section{A.8.5 Normative Bewertungen}

Die Frage, wie das Verhältnis von Parlament und Medien ausfällt, ist nicht zuletzt darum relevant, weil vielleicht nicht jede Form der Wechselwirkungen demokratisch gesehen gut ist. Wie schätzen Sie diesbezüglich die Situation ein? Gibt es demokratisch gesehen problematische Dinge?

- Sichtbarkeit der parlamentarischen Arbeit

- Strategische Partnerschaften: Partikulare Interessen vs. Interessen der Wählerschaft

- Journalistisches Selbstverständnis

- Selbstverständnis der ParlamentarierInnen

- Wer ist «schuld»: Medien oder Parlament?

\section{A.8.6 Entwicklung}

Sie haben ausführlich beschrieben, wie Sie die gegenwärtige Situation einschätzen. Was würden Sie zur Entwicklung der Situation sagen: Gab es in den letzten ca. 10 bis 20 Jahren Ihrer Meinung nach Veränderungen?

- Umgang mit Medien allgemein

- Medialisiertheit der ParlamentarierInnen

- Gegenseitige Beeinflussung von Medien und Parlament 
- Nach unterschiedlichen Arenen

- Strategischer Umgang miteinander

- Wandel der zu erreichenden Ziele

\section{A.8.7 Abschluss}

Gibt es vielleicht noch Punkte, die wir nicht angesprochen haben, die aber wichtig sind? 